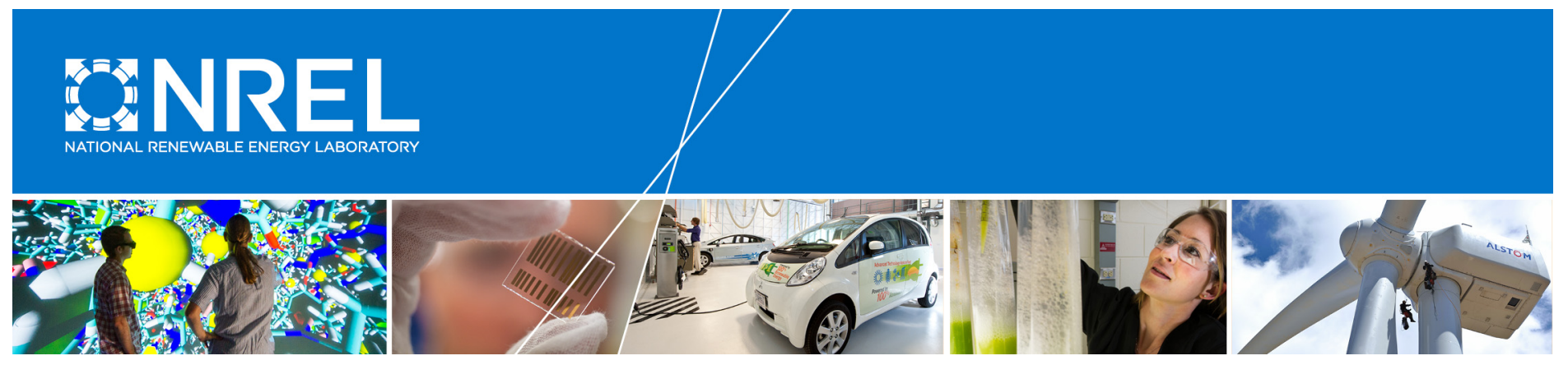

\title{
NREL Integrate: \\ RCS-4-42326
}

\section{June 2015 - December 2016}

Jim Waight and Shailendra Grover

OMNETRIC

Palo Alto, California

Stuart Laval

Duke Energy

Palo Alto, California

Les Sheppard

University of Texas San Antonio

San Antonio, Texas

James Boston

CPS Energy

San Antonio, Texas

NREL Technical Monitor: Andrew Hudgins

NREL is a national laboratory of the U.S. Department of Energy Office of Energy Efficiency \& Renewable Energy Operated by the Alliance for Sustainable Energy, LLC

This report is available at no cost from the National Renewable Energy Laboratory (NREL) at www.nrel.gov/publications.

\section{Subcontract Report}

NREL/SR-5B00-70276

February 2018

Contract No. DE-AC36-08GO28308 


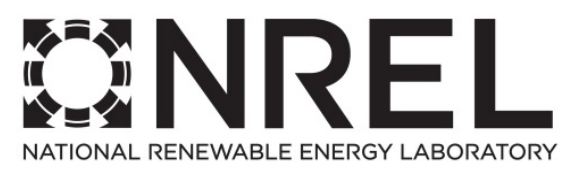

\title{
NREL Integrate: \\ RCS-4-42326
}

\section{June 2015 - December 2016}

\author{
Jim Waight and Shailendra Grover \\ OMNETRIC \\ Palo Alto, California
}

Stuart Laval

Duke Energy

Palo Alto, California

Les Sheppard

University of Texas San Antonio

San Antonio, Texas

James Boston

CPS Energy

San Antonio, Texas

NREL Technical Monitor: Andrew Hudgins

Prepared under Subcontract No. NCS-5-42326-01.

NREL is a national laboratory of the U.S. Department of Energy

Office of Energy Efficiency \& Renewable Energy

Operated by the Alliance for Sustainable Energy, LLC

This report is available at no cost from the National Renewable Energy Laboratory (NREL) at www.nrel.gov/publications.

National Renewable Energy Laboratory 15013 Denver West Parkway

Golden, CO 80401

303-275-3000 • www.nrel.gov
Subcontract Report

NREL/SR-5B00-70276

February 2018

Contract No. DE-AC36-08G028308 


\title{
This publication was reproduced from the best available copy submitted by the subcontractor and received no editorial review at NREL.
}

\begin{abstract}
NOTICE
This report was prepared as an account of work sponsored by an agency of the United States government. Neither the United States government nor any agency thereof, nor any of their employees, makes any warranty, express or implied, or assumes any legal liability or responsibility for the accuracy, completeness, or usefulness of any information, apparatus, product, or process disclosed, or represents that its use would not infringe privately owned rights. Reference herein to any specific commercial product, process, or service by trade name, trademark, manufacturer, or otherwise does not necessarily constitute or imply its endorsement, recommendation, or favoring by the United States government or any agency thereof. The views and opinions of authors expressed herein do not necessarily state or reflect those of the United States government or any agency thereof.
\end{abstract}

This report is available at no cost from the National Renewable Energy Laboratory (NREL) at www.nrel.gov/publications.

Available electronically at SciTech Connect http:/www.osti.gov/scitech

Available for a processing fee to U.S. Department of Energy and its contractors, in paper, from:

\author{
U.S. Department of Energy \\ Office of Scientific and Technical Information \\ P.O. Box 62 \\ Oak Ridge, TN 37831-0062 \\ OSTI http://www.osti.gov \\ Phone: 865.576.8401 \\ Fax: 865.576.5728 \\ Email: reports@osti.gov
}

Available for sale to the public, in paper, from:

\author{
U.S. Department of Commerce \\ National Technical Information Service \\ 5301 Shawnee Road \\ Alexandria, VA 22312 \\ NTIS http://www.ntis.gov \\ Phone: 800.553 .6847 or 703.605 .6000 \\ Fax: 703.605.6900 \\ Email: orders@ntis.gov
}




\section{OMNETRIC Group $_{\text {G }}$}

\section{A Siemens \& Accenture Company}

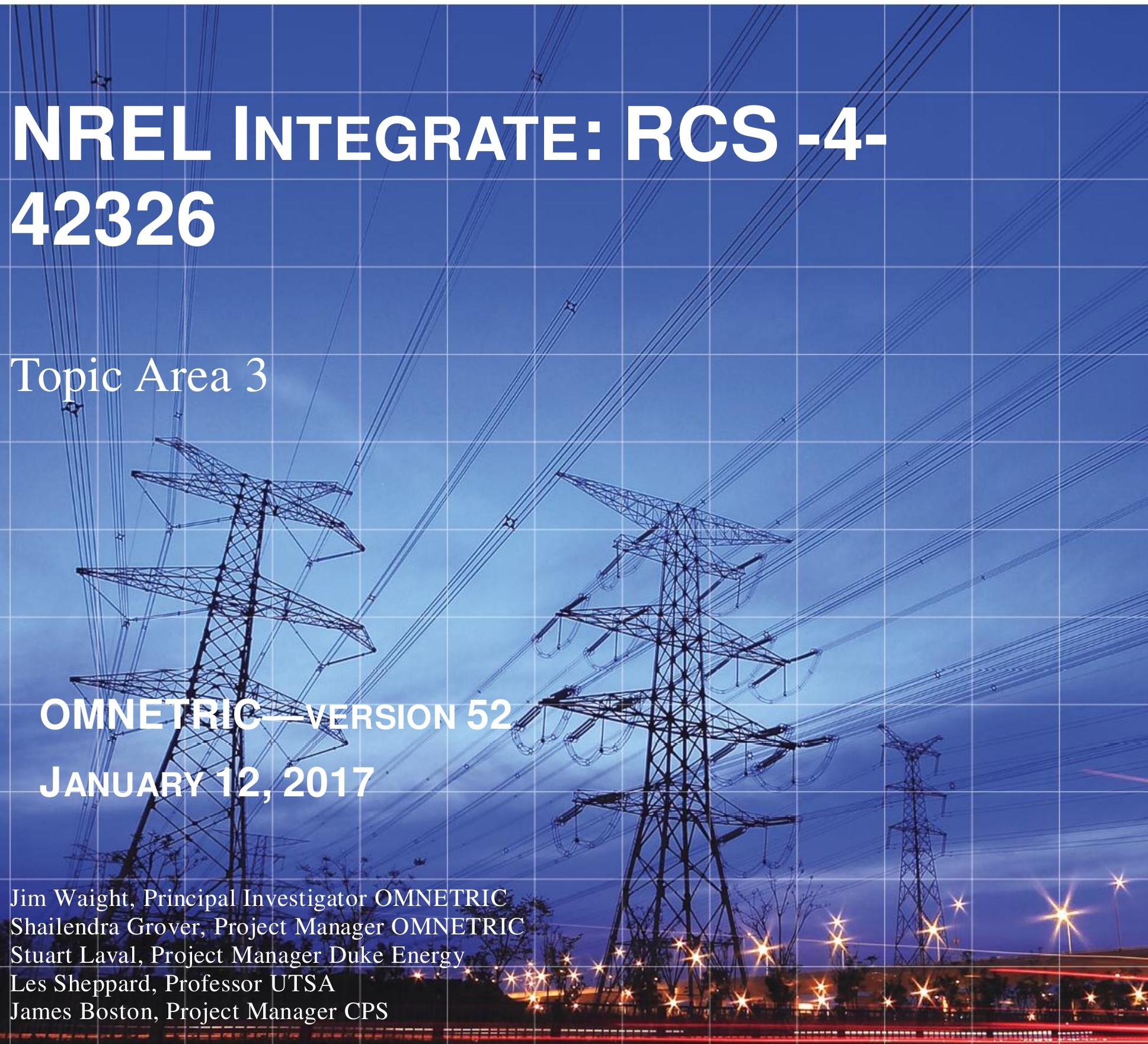

James Boston, Project Manager CPS 


\title{
OMNETRIC Corporation
}

Jim. Waight, Principal Investigator

Shailendra Grover, Project Manager

Ray Lui

Jose Mathew

Duke Energy

Stuart Laval, Project Manager

Dwayne Bradley

David Lawrence

\author{
CPS Energy \\ James Boston, Project Manager \\ Mike Sparkman
}

\author{
University of Texas San Antonio \\ Les Sheppard, Professor \& Project Manager Bin \\ Dong, Professor \\ Jim Richardson, Professor
}

\section{Siemens}

Shashank Pande, Architect

\section{NREL ESIF}

Andrew Hudgins, Project Manager

Greg Martin, ESIF Engineering Group Manager 


\section{Table of Contents}

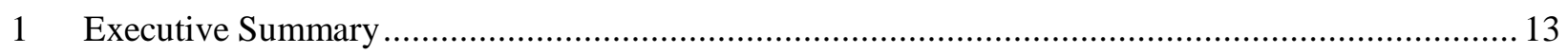

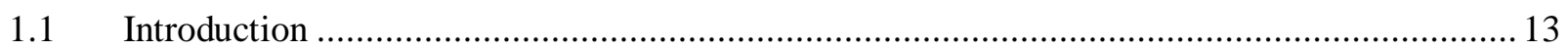

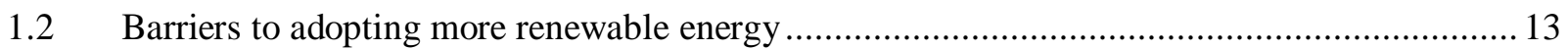

$1.3 \quad$ Microgrid as an element of the power system control hierarchy ….................................... 14

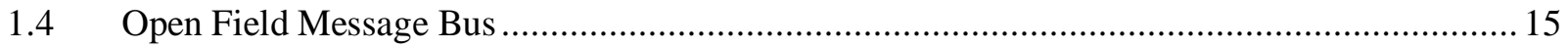

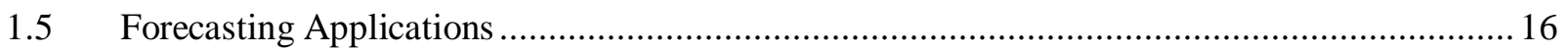

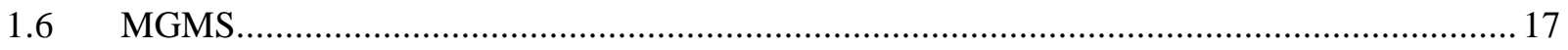

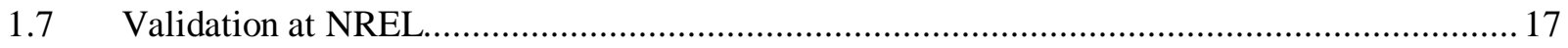

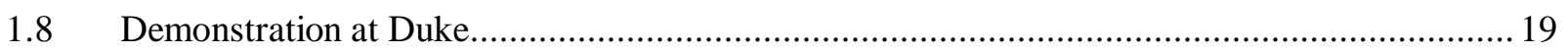

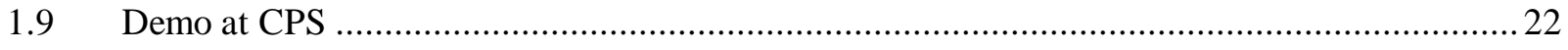

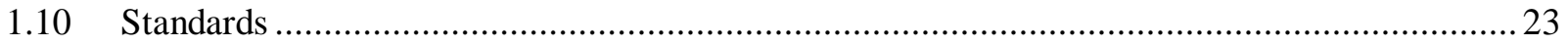

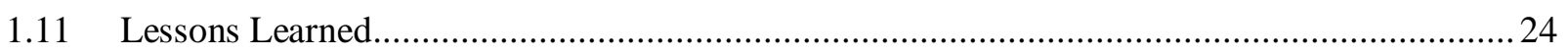

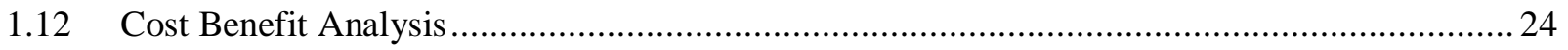

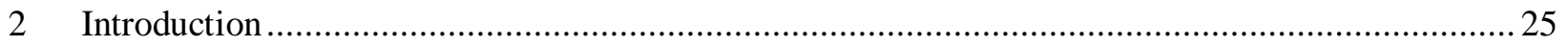

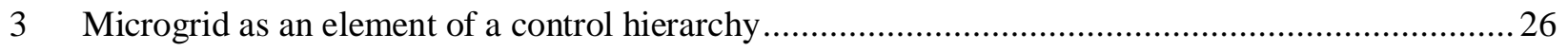

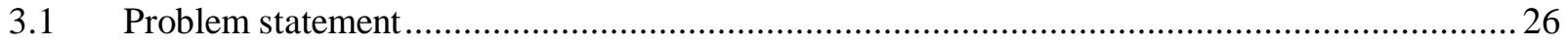

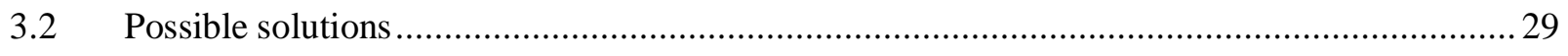

3.2.1 Adjustment of Production from Renewables ..................................................... 29

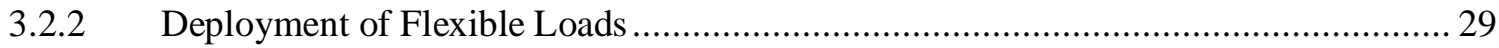

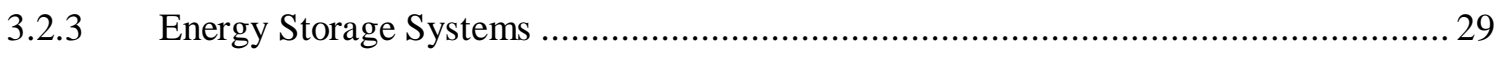

3.2.4 Reserves Based on Different Technologies ...................................................... 30

3.2.5 Increasing Penetration of Renewable Resources Exacerbate the Problem of Balancing30

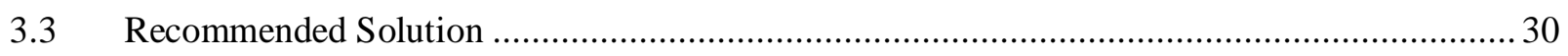

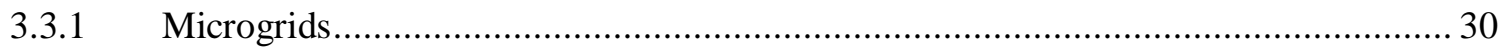

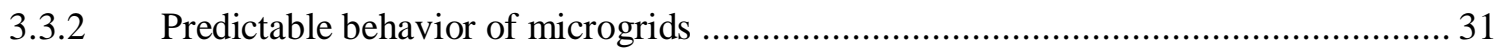

3.3.3 Response of Utility System Operator ........................................................... 32

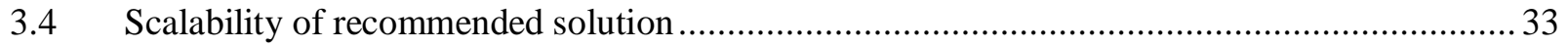

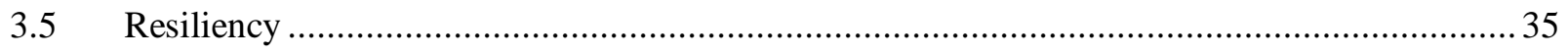

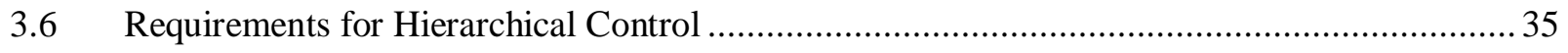

3.6.1 Improved communications, control, and Interoperability ..................................... 35 


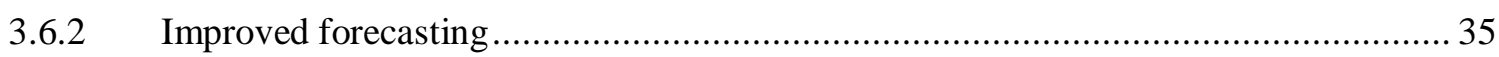

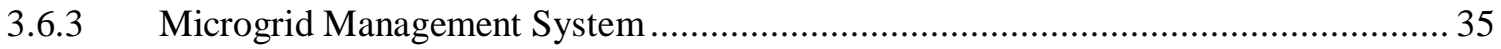

4 Open FMB

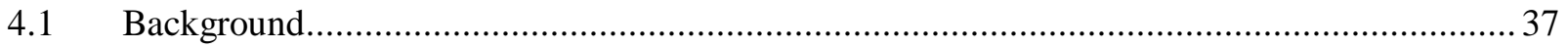

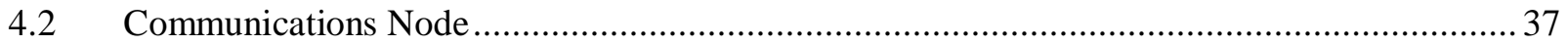

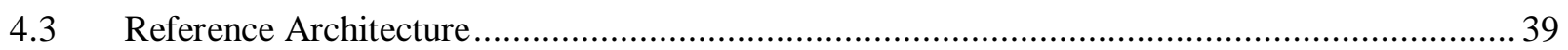

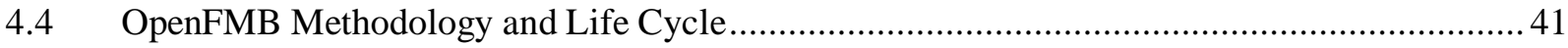

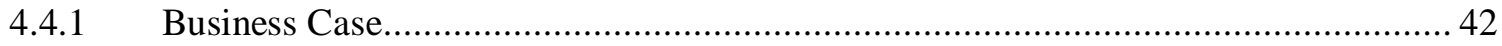

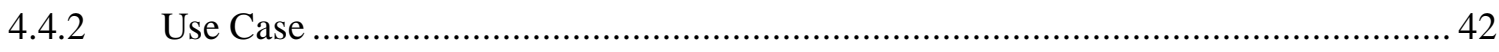

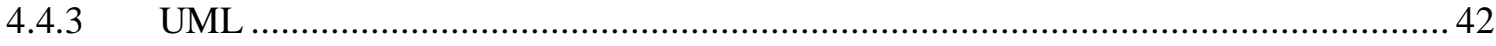

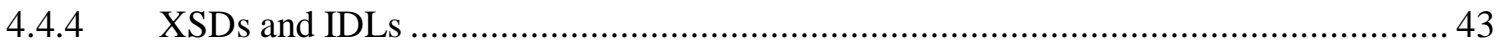

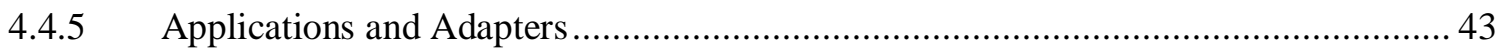

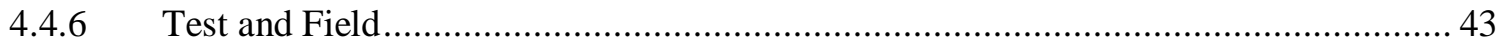

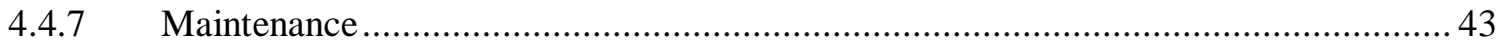

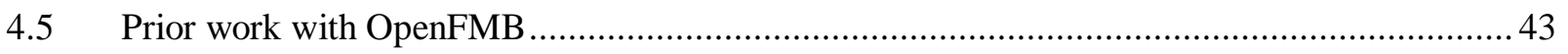

4.6 Application of OpenFMB to Enhancing the Ability of the Grid to Support Renewables .......... 44

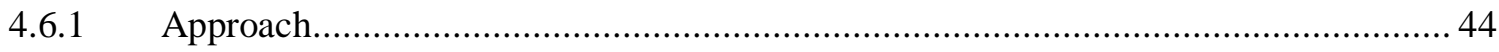

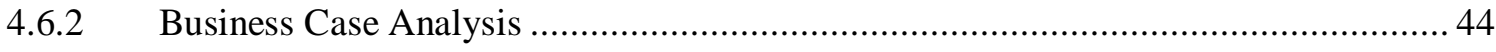

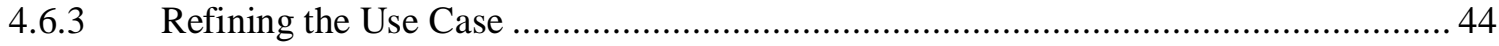

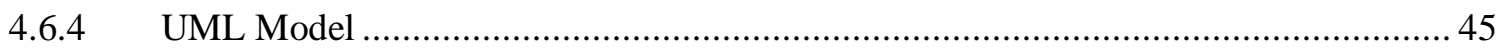

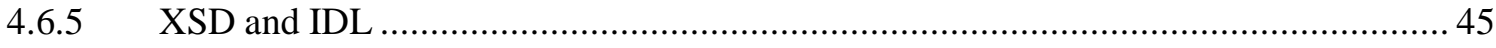

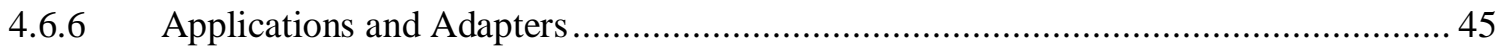

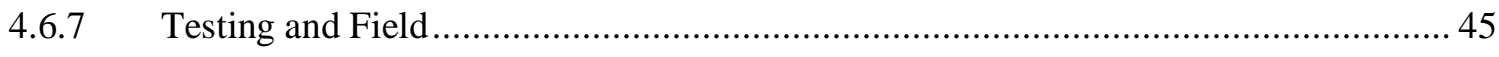

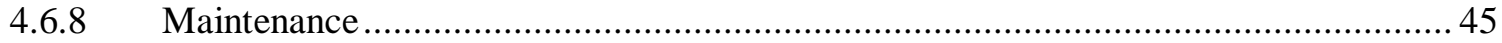

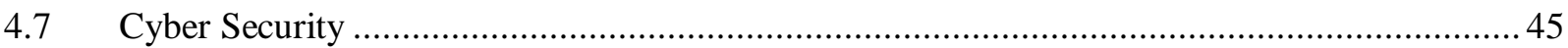

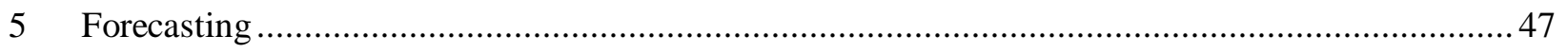

5.1 Current State of Art - Load and PV Production Forecasting ........................................... 47

5.1.1 Load forecasting and day-ahead PV production forecasting .................................. 47

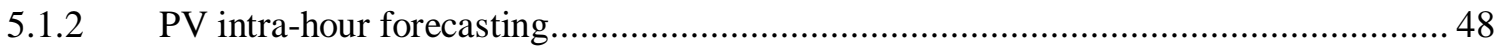

$5.2 \quad$ UTSA Advanced Load and Solar Forecasting Methodology .............................................. 51 


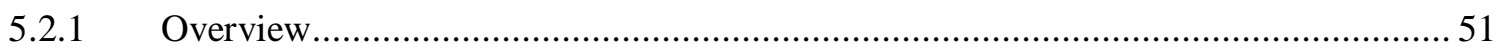

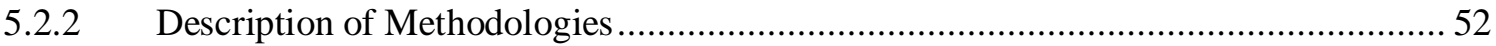

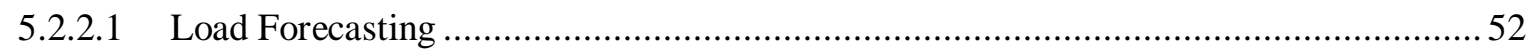

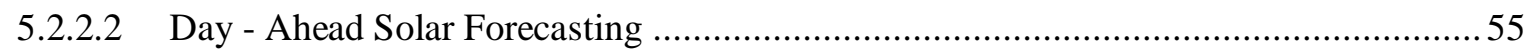

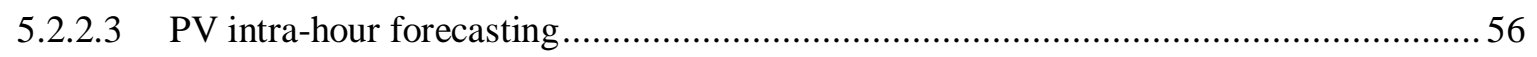

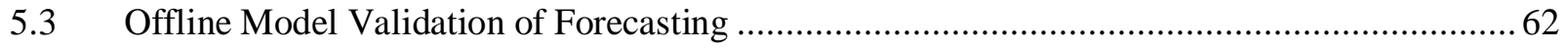

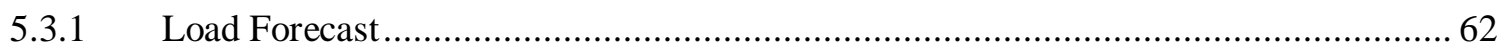

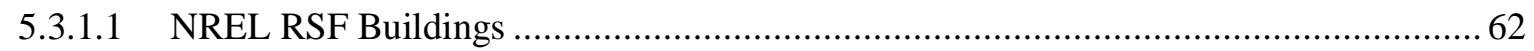

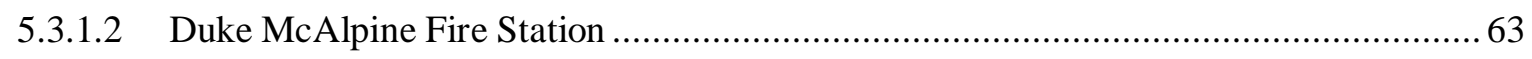

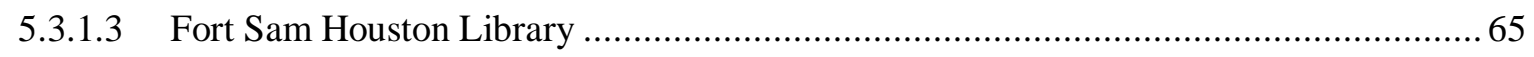

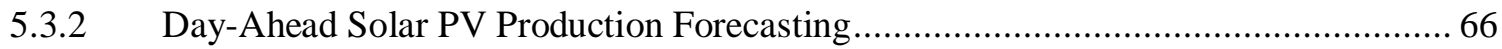

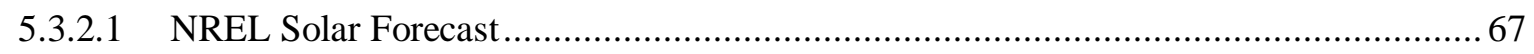

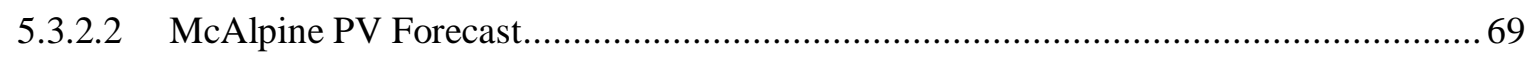

5.3.2.3 Fort Sam Houston.................................................................................................. 70

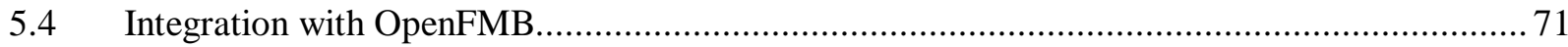

5.5 Description of the Online Deployment Platform ........................................................ 75

5.5.1 Load forecasting and day-ahead PV production forecasting .................................. 75

5.5.2 Sky Imager Intra-hour Forecasting at NREL …................................................... 76

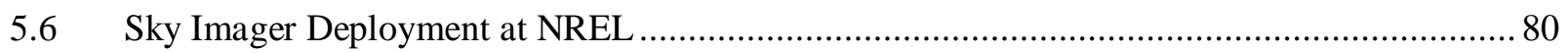

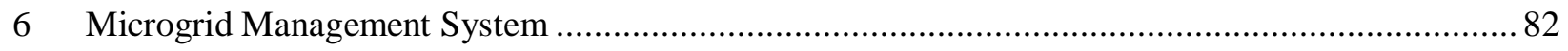

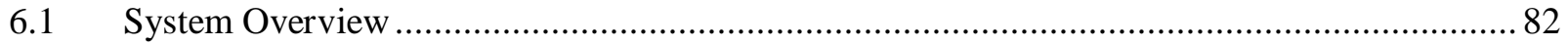

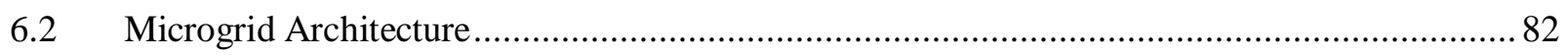

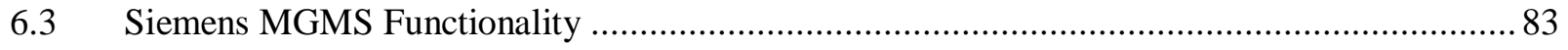

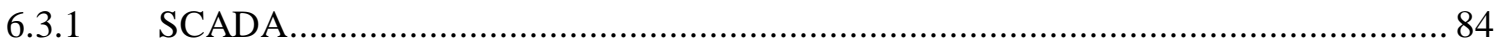

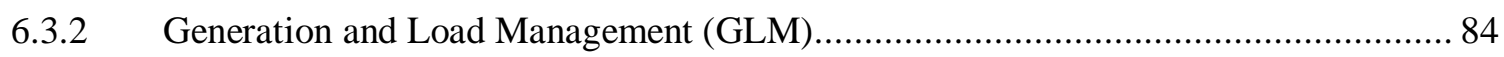

6.3.3 Generation Forecast and Optimization …............................................................ 84

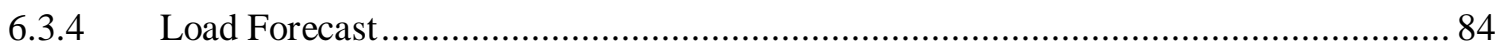

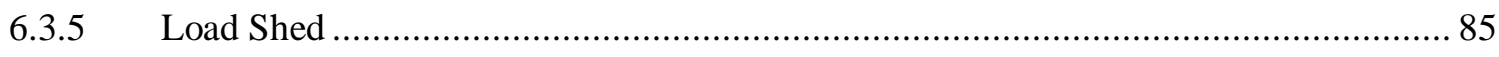

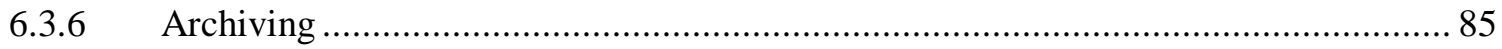




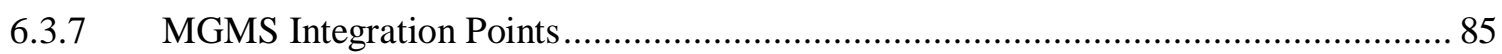

6.4 Integration of the Siemens MGMS with the OpenFMB architecture ................................... 85

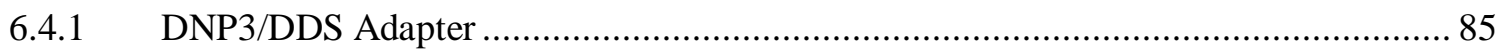

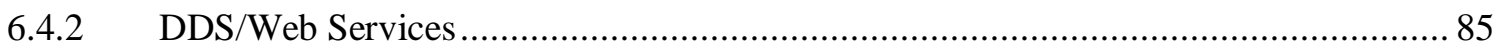

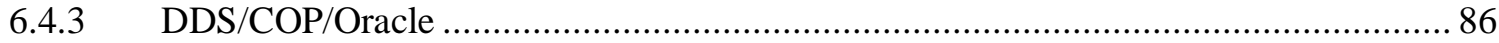

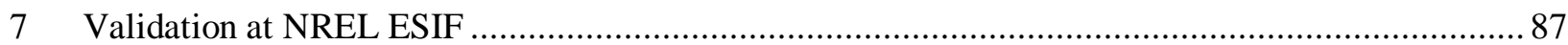

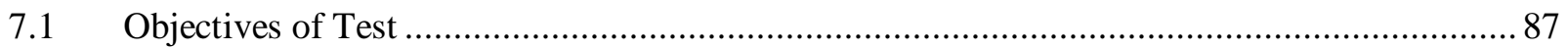

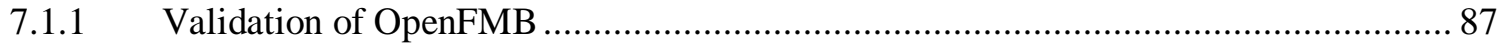

7.1.2 Validation of the Siemens MGMS ability to control a generic microgrid.................. 87

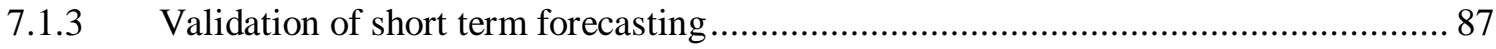

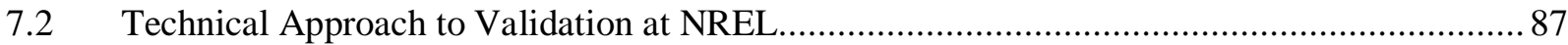

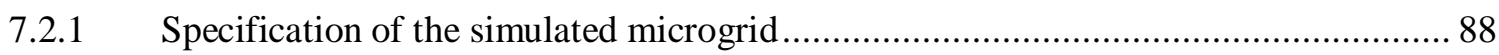

7.2.2 Development of the Control System Architecture ............................................... 88

7.2.3 Component Interfaces Information Exchange Modeling ......................................... 88

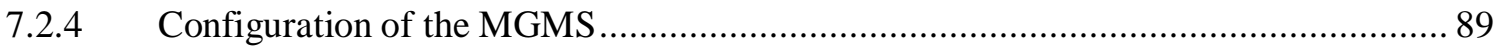

7.2.5 Development of the Frame Test Specification ...................................................... 89

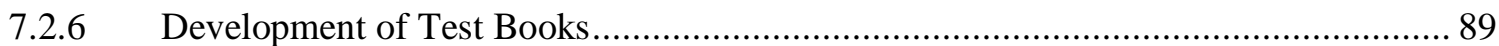

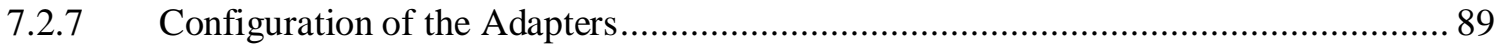

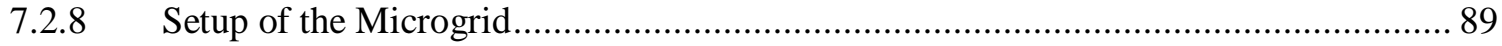

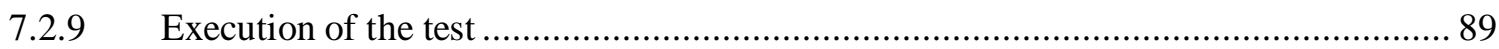

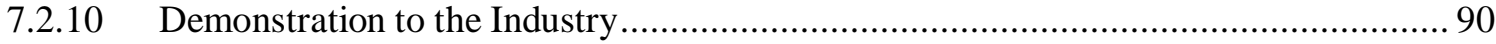

7.3 Configuration of NREL Equipment into a Simulated Microgrid........................................... 90

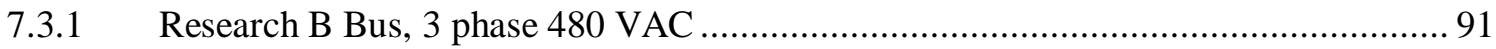

7.3.2 The Erigo Eagle Picher 300 kW Battery Energy Storage System............................. 91

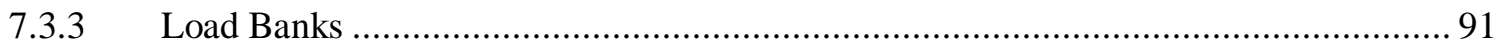

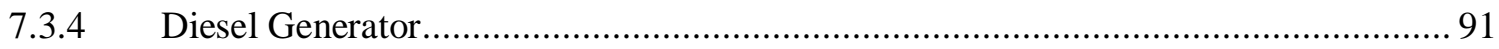

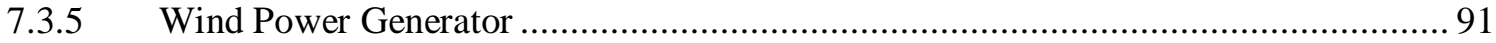

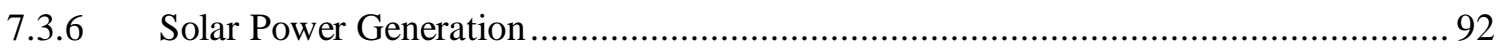

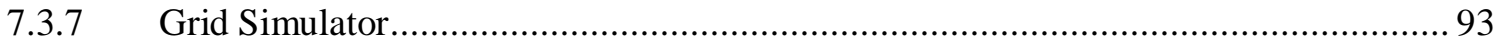

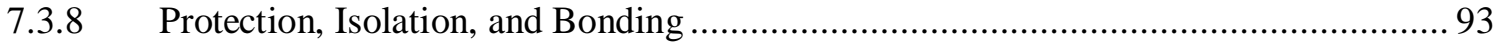




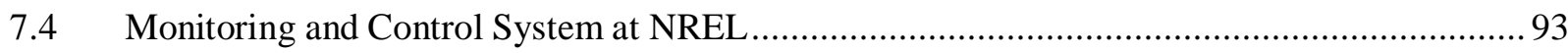

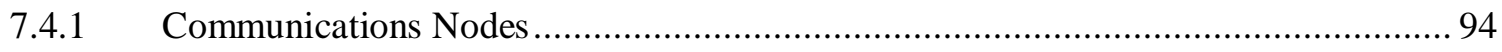

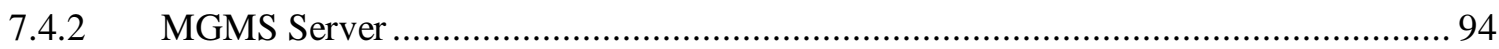

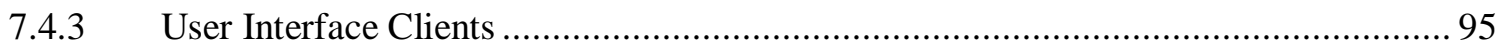

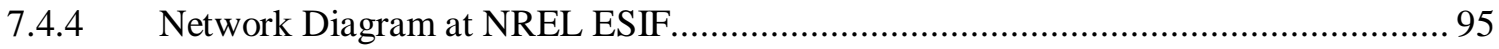

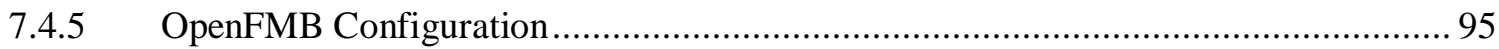

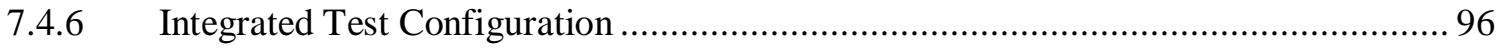

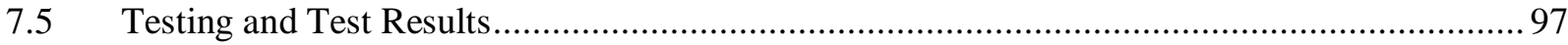

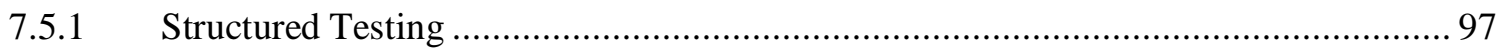

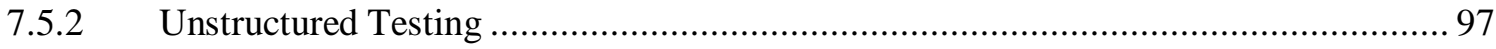

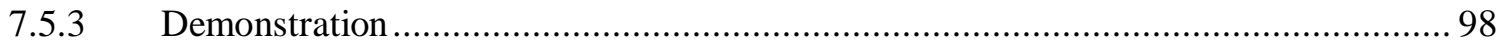

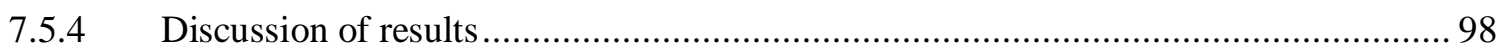

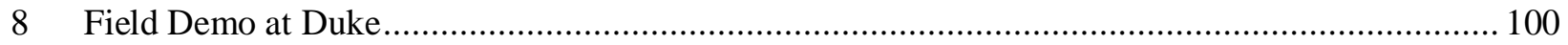

8.1 Duke Energy Microgrid at Mt Holly …................................................................................ 100

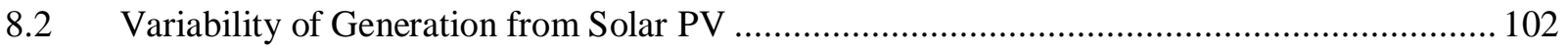

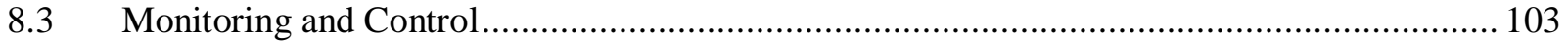

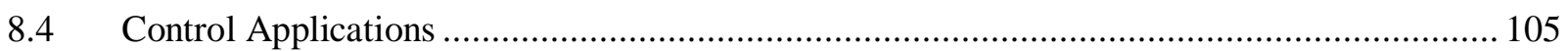

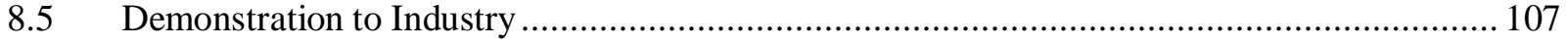

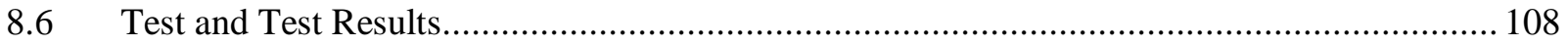

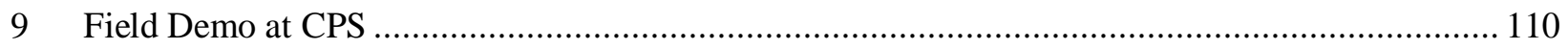

$9.1 \quad$ CPS Energy Microgrid at Joint Base San Antonio …..................................................... 110

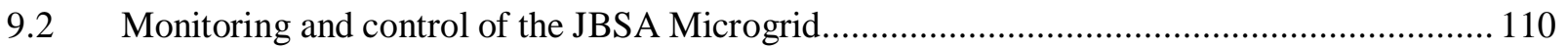

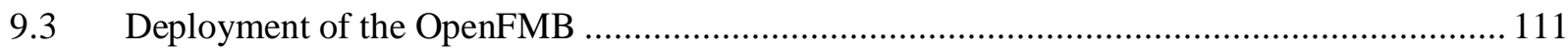

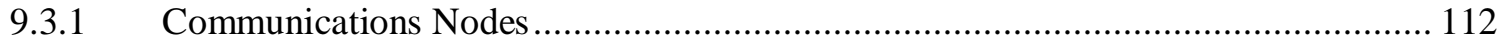

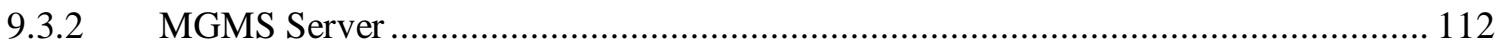

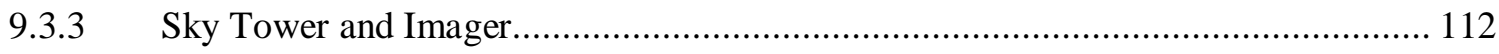

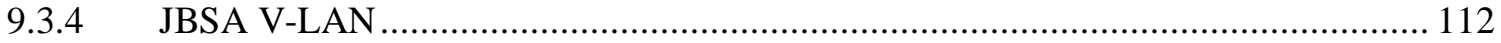

9.4 Sky Imager System Deployment at Fort Sam Houston Library........................................ 113

9.4.1 Load forecasting and day-ahead PV production forecasting ................................. 115

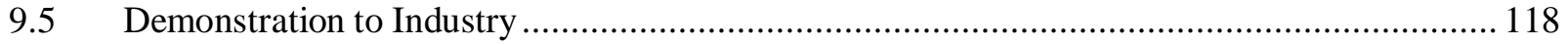




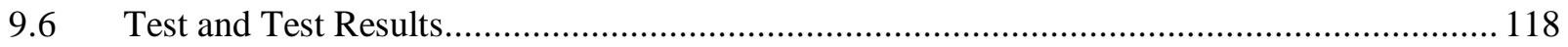

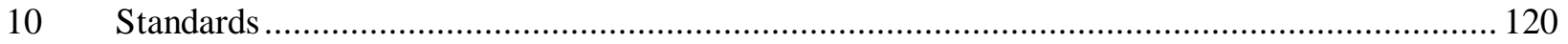

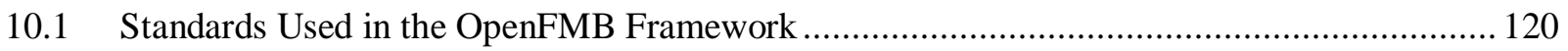

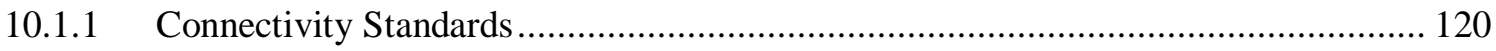

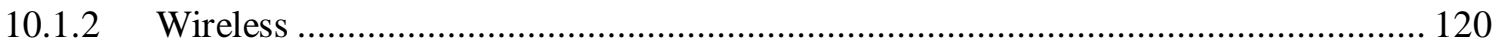

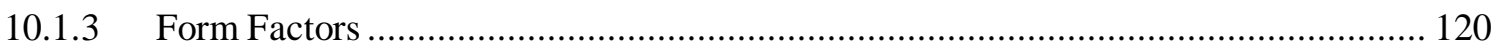

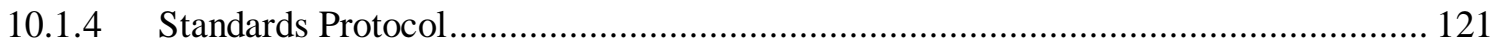

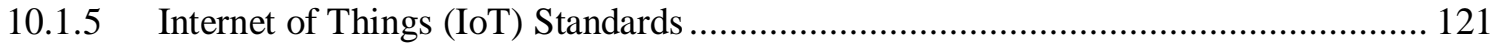

10.1.6 Semantic Data Model Standards ...................................................................... 121

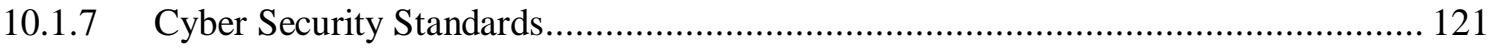

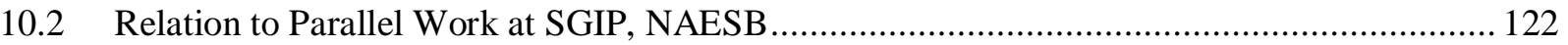

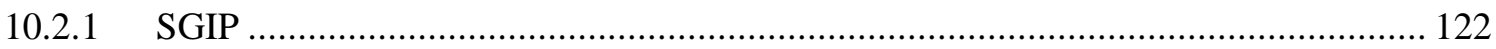

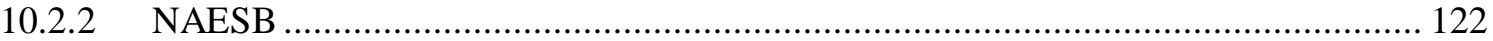

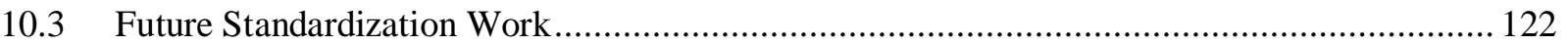

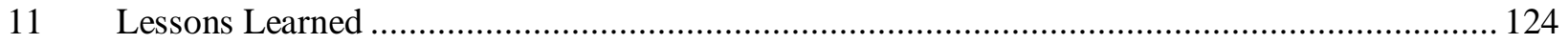

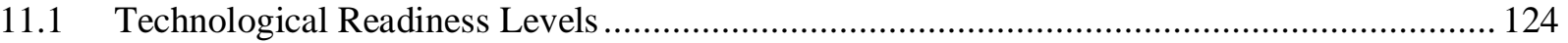

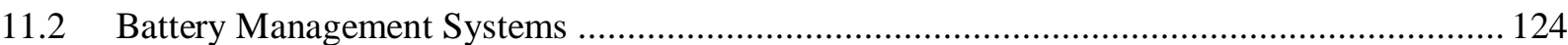

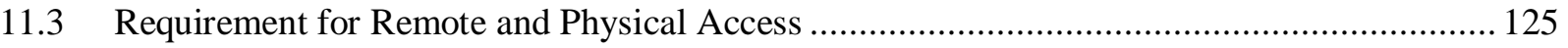

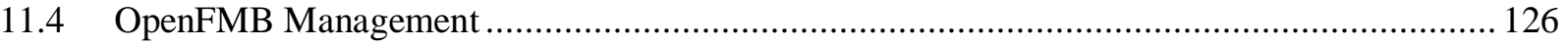

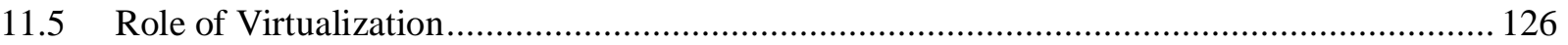

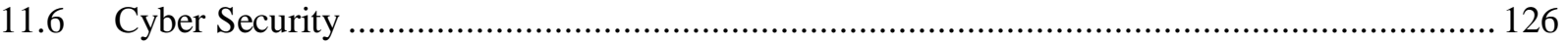

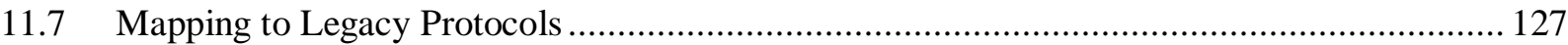

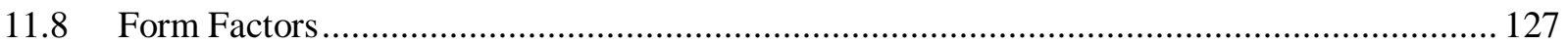

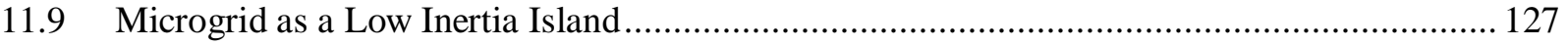

11.10 Changes in Design and Operational Standards at Utilities ........................................... 128

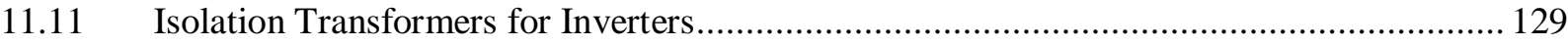

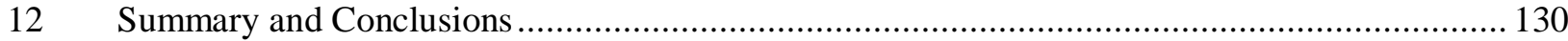

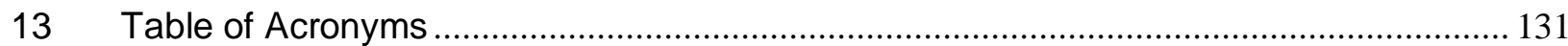

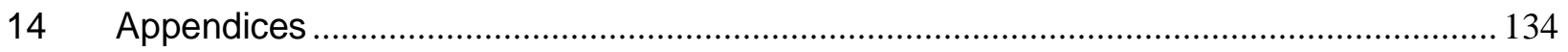

14.1 Appendix A Use Case 1: Optimization of Microgrid........................................................... 134 
14.1.1 Optimization Use Case Background.............................................................. 134

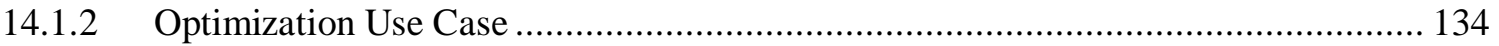

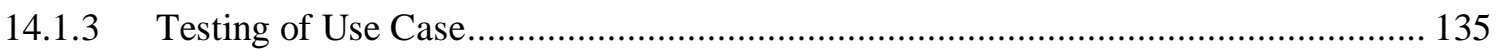

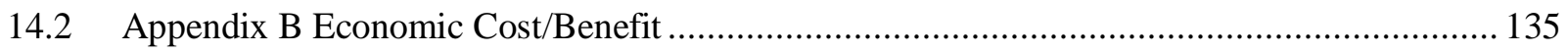

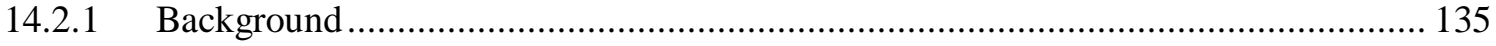

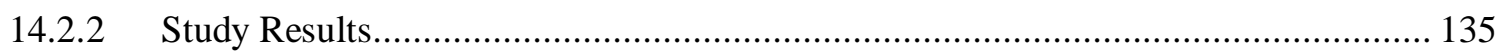

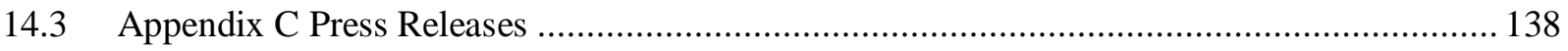

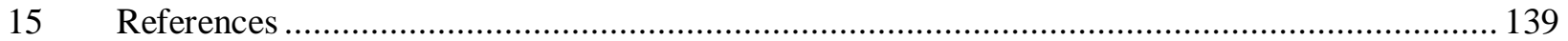




\section{Table of Figures}

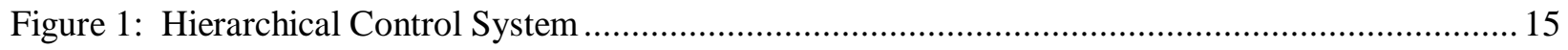

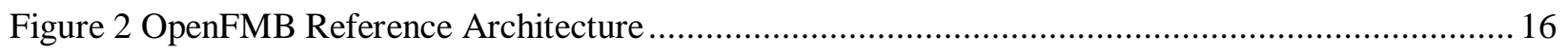

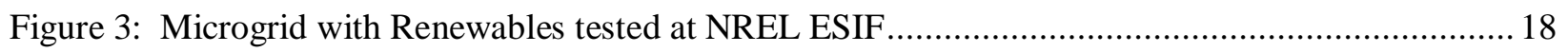

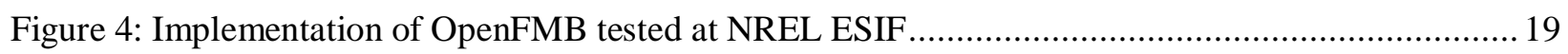

Figure 5: Implementation of OpenFMB tested at Mt Holly Training Center .........................................2 21

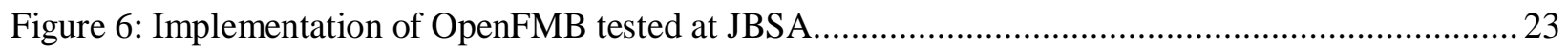

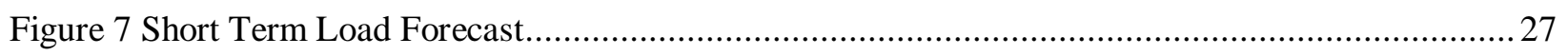

Figure 8: Short Term Solar PV Generation Forecast...................................................................28

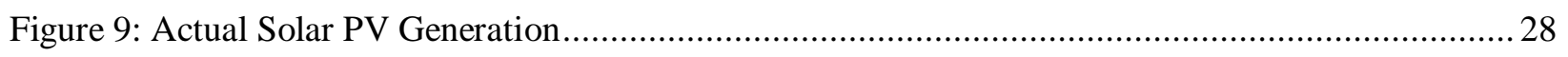

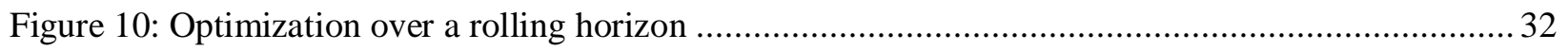

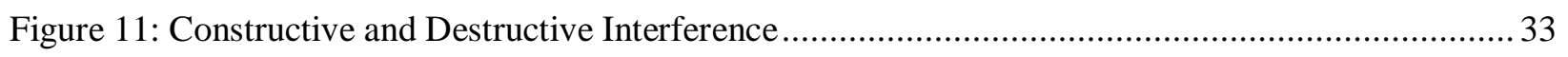

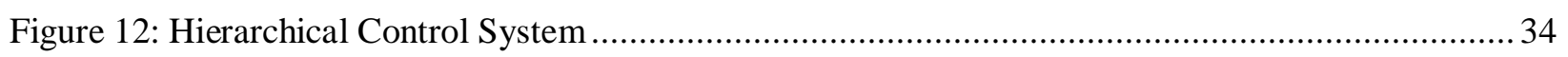

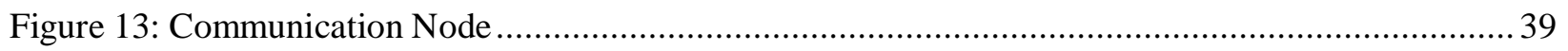

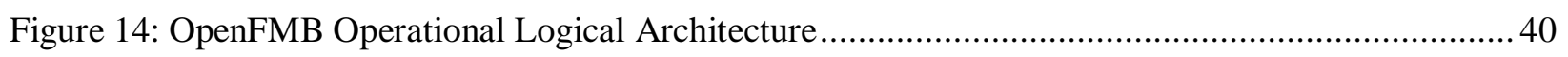

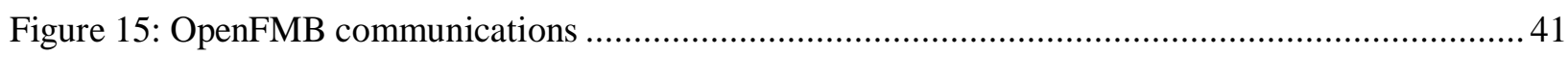

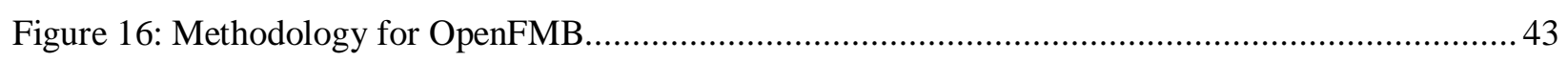

Figure 17 Typical GOES West Image of Texas......................................................................... 48

Figure 18 Comparison between satellite irradiance estimates and those obtained from a solar panel placed

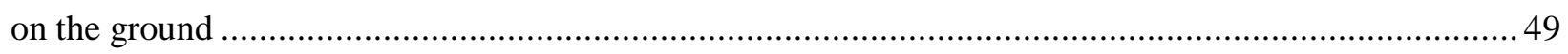

Figure 19: (a) Image of a typical sky imager, (b) scheme of a typical sky imager; and (c) artifacts placed in the image due to the camera and the shadow band used to avoid direct reflection of the sun on the

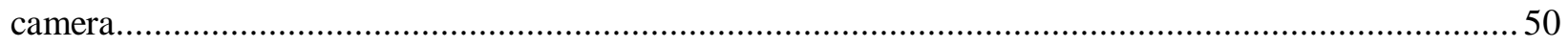

Figure 20: Typical sky image of a partly cloudy day (a) before; and (b) after masking the obstructions .. 50 Figure 21: (a) Typical ceilometer and (b) scheme the calculation of cloud lower height........................ 51

Figure 22: General flow of information and analyses for the UTSA solar and building load forecast

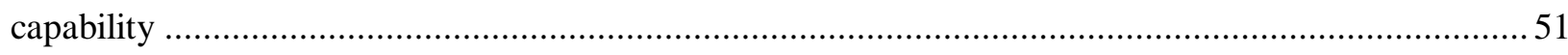

Figure 23: Scheme of the building energy forecasting methodology developed by UTSA.....................53

Figure 24: RSF Building Load at different time periods .....................................................................5

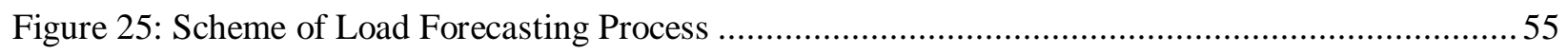

Figure 26: Scheme of the day ahead solar forecasting methodology developed by UTSA.....................55

Figure 27: Scheme of the load forecasting process .....................................................................56

Figure 28: (a) View of the UTSA Sky Imager placed on the roof of a house and 9b0 scheme of the UTSA sky imager and main components and alternatives ......................................................................5 57

Figure 29 Scheme of the treatment of on sky image using the Fusion Technique developed by UTSA....57 Figure 30: Sky Image (a) before; and (b) after the transformation into a Cartesian plan image. (c) representation of the transformation process (top to bottom) .......................................................5

Figure 31 Vectorization of the individual cloud motion by analyzing consecutive sky images ...............58

Figure 32: Cloud match results from current time for different clouds ............................................59 
Figure 33: True North orientation based on physical location and 5-min vector forecast based on previous vectors from feature points

Figure 34: Scheme of the tracing procedure to provide a pixel intensity value from the sky image for any point on the ground.

Figure 35: Red to blue ratio calibration normalized (0-255). In a clear sky blue is much larger than read, and in a cloudy sky blue is more equal to red 60

Figure 36: Scheme of the Monte Carlo Simulation 61

Figure 37: Capture of the user interface provided by UTSA for the short term forecasting system 61

Figure 38: The fire station variance compared to NREL in a normalized score for 15 minute resolution . 65 Figure 39: Comparison of measured and forecasted energy consumption for the Fort Sam Houston Library 66

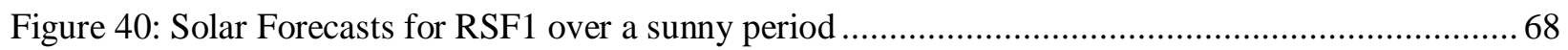

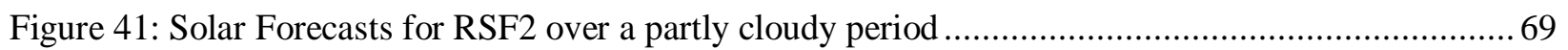

Figure 42: Fort Sam Houston PV Forecasting during sunny days .................................................. 71

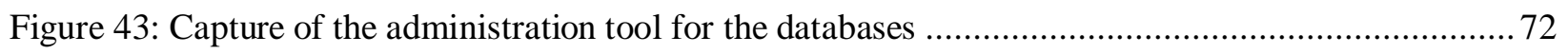

Figure 44: A synchronization scheme implemented through OpenFMB .............................................. 72

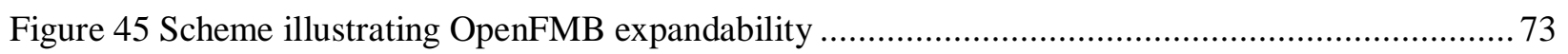

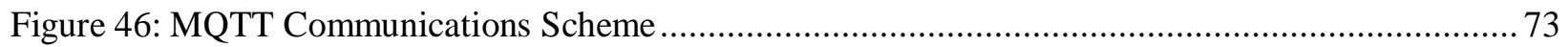

Figure 47: Screen capture of the OpenFMB process when input data is provided and forecasting is

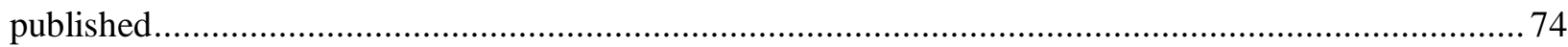

Figure 48: Scheme of the suggested deployment in the MGMS .................................................... 75

Figure 49: Screen capture showing an extract of the routines for forecasting ...................................... 76

Figure 50: Screen capture showing how the forecasting tools are provided ........................................ 76

Figure 51: NREL site instrumentation for the sky imager, MET tower and PV plants...........................77

Figure 52: (a) Current cloud cover at NREL; and (b) cloud cover over NREL 5-min ahead. Note: clear

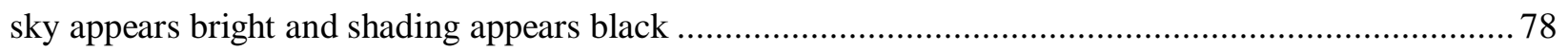

Figure 53: Direct Solar Irradiance values for five days at NREL. Blue is the measured value during daylight hours and orange is the predicted cloud/no cloud value during the hours 10am - 4 pm.............79 Figure 54: Location of Sky Imager (orange arrow) and the $408 \mathrm{PV}$ plant monitored (yellow arrow) at

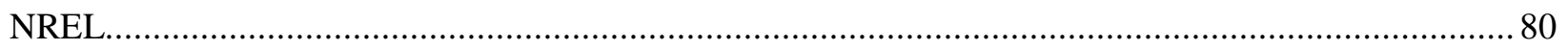

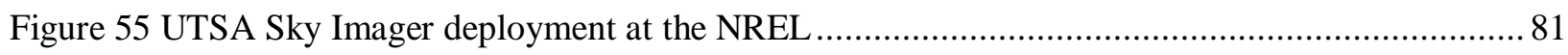

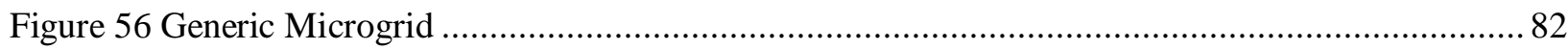

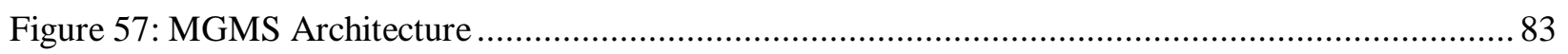

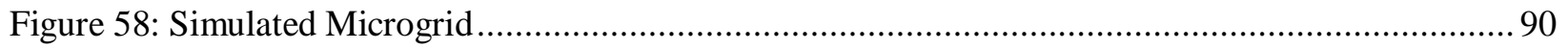

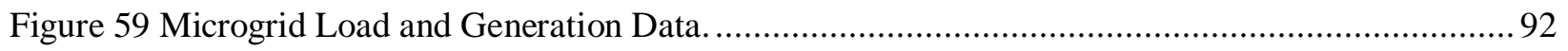

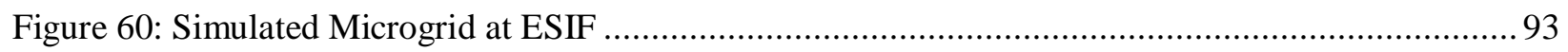

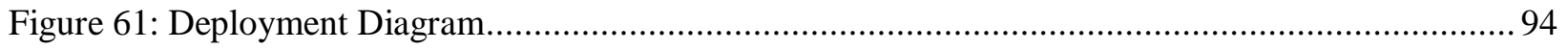

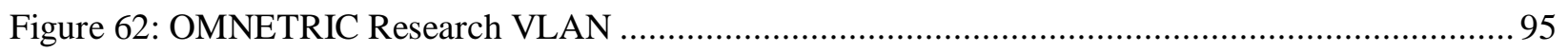

Figure 63 Microgrid Power System with Metering and Communications Interfaces ............................96

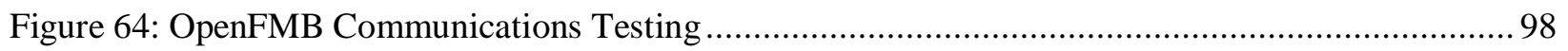




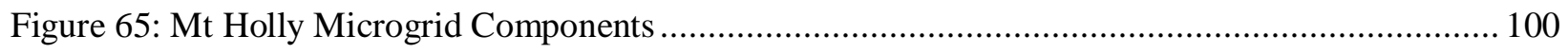

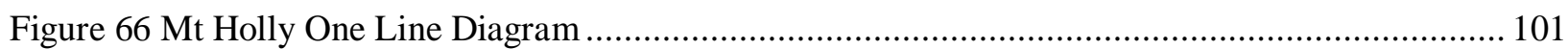

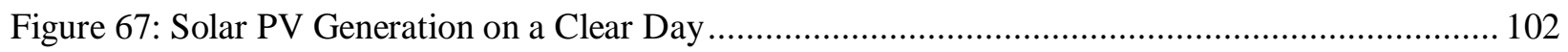

Figure 68 Solar PV Generation on a Cloudy Day with Rapidly Moving Sirius Clouds......................... 103

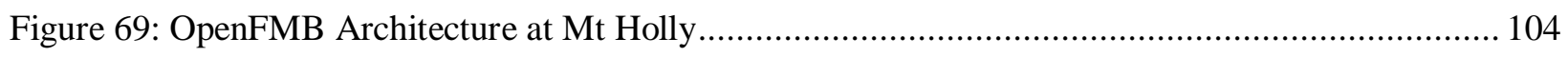

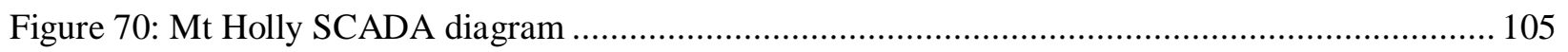

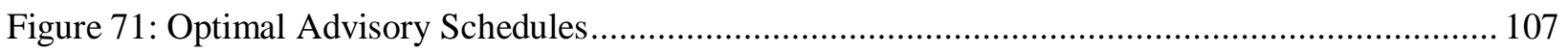

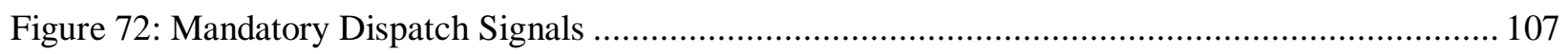

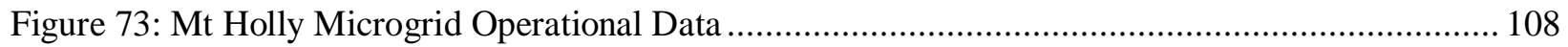

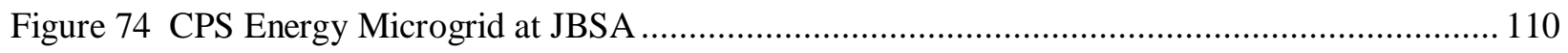

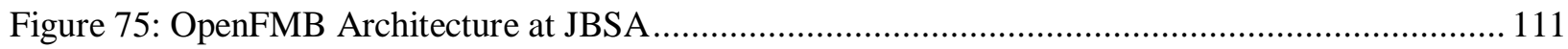

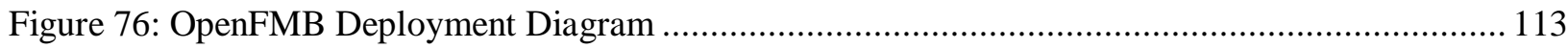

Figure 77: Location and layout of the Fort Sam Houston Library deployment .................................... 114

Figure 78: Internal Components of the Campbell Scientific Box with the battery, MyRio and transformer

Figure 79: Comparison of measured PV production and ASOS cloud cover forecast for a partly cloudy

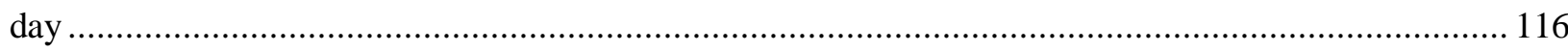

Figure 80: Comparison of measured PV production and ASOS cloud cover forecast for a cloudy day on

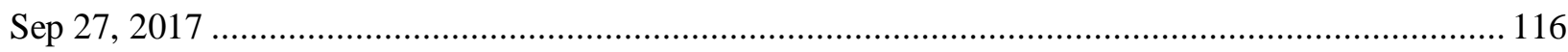

Figure 81: Comparison of measured PV production and ASOS cloud cover forecast for a cloudy day at

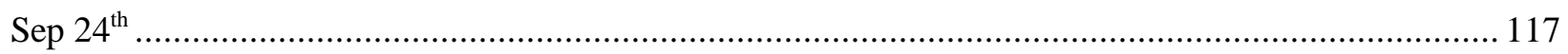

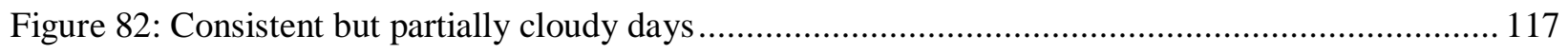

Figure 83: Inconsistent days with mixed cloudy and sunny days .................................................. 118

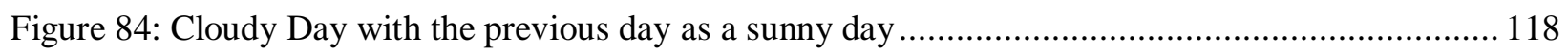

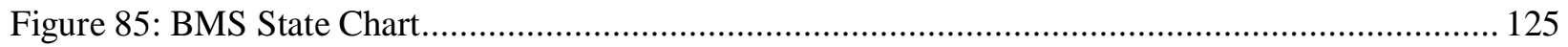




\section{Executive Summary}

\subsection{Introduction}

OMNETRIC Corp., Duke Energy, CPS Energy, and the University of Texas at San Antonio (UTSA) created a project team to execute the project NREL Integrate RCS-4-42326, Topic Area 3: Integrated Systems: "OpenFMB Reference Architecture Demonstration." The project included development and demonstration of concepts that will enable the electric utility grid to host larger penetrations of renewable resources. The project concept calls for the aggregation of renewable resources and loads into microgrids and the control of these microgrids with an implementation of the OpenFMB Reference Architecture.

\subsection{Barriers to adopting more renewable energy}

The production of power from the renewable resources that are appearing on the grid today is very closely linked to the weather. The difficulty of forecasting the weather, which is well understood, leads to difficulty in forecasting the production of renewable resources. The current state of the art in forecasting the power production from renewables (solar PV and wind) are accuracies in the range of 12-25 percent NMAE. In contrast the demand for electricity aggregated to the system level, is easier to predict. The state of the art of demand forecasting done, 24 hours ahead, is about 2-3\% MAPE. Forecasting the load to be supplied from conventional resources (demand minus generation from renewable resources) is thus very hard to forecast. This means that even a few hours before the time of consumption, there can be considerable uncertainty over what must be done to balance supply and demand.

Adding to the problem of difficulty of forecasting, is the reality of the variability of the actual production of power from renewables. Due to the variability of wind speeds and solar insolation, the actual output of power from renewable resources can vary significantly over a short period of time. Gusts of winds result is variation of power output of wind turbines. The shadows of clouds moving over solar PV arrays result in the variation of power production of the array. This compounds the problem of balancing supply and demand in real time.

Establishing a control system that can manage distribution systems with large penetrations of renewable resources is difficult due to two major issues: (1) the lack of standardization and interoperability between the vast array of equipment in operation and on the market, most of which use different and proprietary means of communication and (2) the magnitude of the network and the information it generates and consumes.

The objective of this project is to provide the industry with a design concept and tools that will enable the electric power grid to overcome these barriers and support a larger penetration of clean energy from renewable resources. 


\subsection{Microgrid as an element of the power system control hierarchy}

The electric grid in North America is a multi-level hierarchical system with a (possible) Independent System Operator/Regional Transmission Operator (ISO/RTO) level managed by an Energy Management System (EMS) and an Energy Market Management System (EMM); a utility level managed by an EMS; and one or more levels of utility Distribution Management System (DMS) systems (Figure 1). Recent innovations have begun to create a microgrid level that consists of small subsets of resources and grid assets including load, renewable resources, conventional resources, demand response, and energy storage systems within clearly defined boundaries which may be disconnected from the larger grid and operated with local supply. Microgrids may be owned by the utility or by some other party. This project proposes the concept of a Microgrid Management System (MGMS) that manages the operation of the microgrid and cooperates with the next level of the control hierarchy by presenting an aggregated, abstracted view of the microgrid to the utility that can be easily incorporated into the DMS and EMS systems that utilities use to manage the grid today. This approach will allow the grid to operate with a very large number of microgrids, each of which may contain large amounts of variable renewable resources, in a fully integrated and reliable manner. 


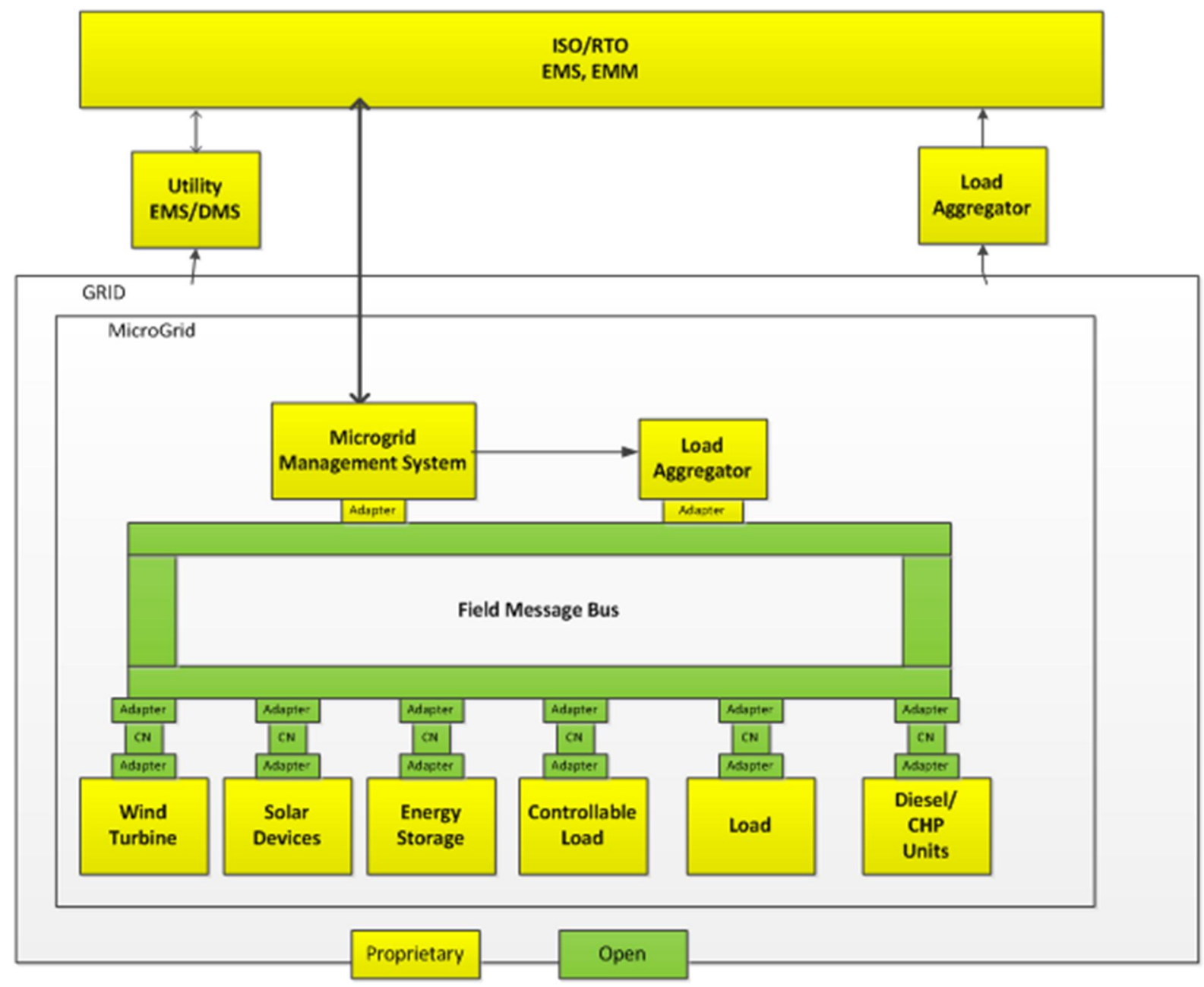

Figure 1: Hierarchical Control System

In the solution recommended by this project, each microgrid forecasts its behavior, and controls its resources to follow its predictions. The MGMS includes a state of the art forecasting application which forecasts the load demand of the microgrid, and the production of power from renewable resources. Using these forecasts as inputs the MGMS optimizes the resources of the microgrid according to a selected optimization objective. For example if the selected objective is to use the most economic resources in a situation where the utility grid has a time-of-use tariff, the optimization may select the use of renewable and storage resources during times of high utility prices and use utility resources during the times when utility prices are lower. A use case was developed that described the information flows associated with the optimization of the resources of the microgrid. This use case was used as the foundation of the Frame Test Specification for testing at the NREL ESIF.

\subsection{Open Field Message Bus}

The OpenFMB is an Architectural Framework and Reference Architecture, shown in Figure 2, which may be used to integrate distributed intelligent nodes to exchange data and interoperate with each other to 
support a large variety of electric utility operations. The OpenFMB extends the concepts of integration of enterprise applications using an enterprise service bus (ESB) to an integration framework that may be applied at the edge of the utility distribution grid as a field message bus to integrate grid edge nodes that acquire and process data locally for control and reporting. It applies concepts from the Industrial Internet Consortium to the electric utility smart grid.

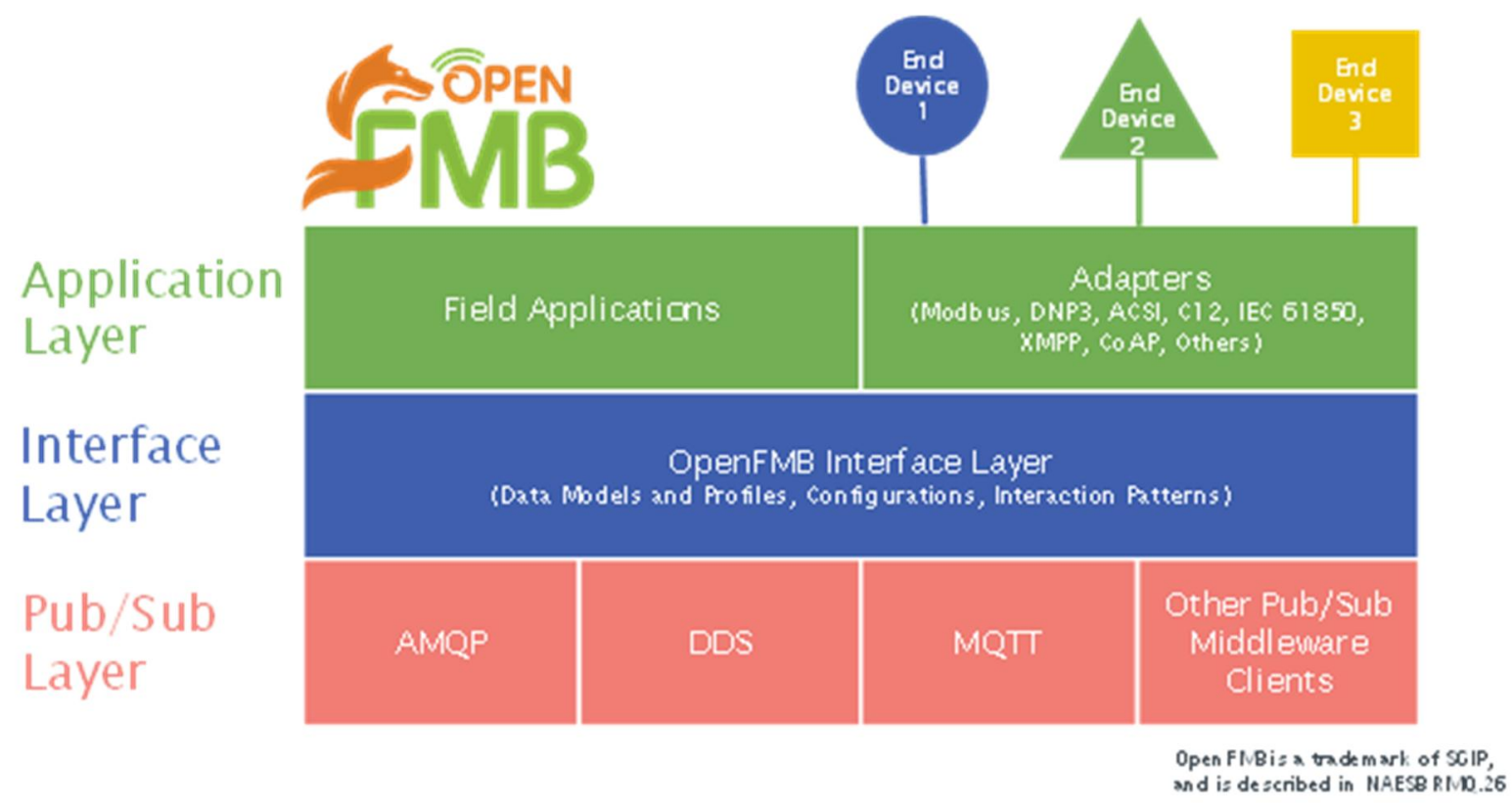

Figure 2 OpenFMB Reference Architecture

Key characteristics of the OpenFMB are: its publish/subscribe integration pattern, a global data space based on a standard semantic model, and its ability to support distributed logic as well as centralized logic. These characteristics enable peer to peer communications and provide a standardized way for grid edge applications to communicate and access data. The use of the standard semantic model enables the development of adapters to gain access to power system data from legacy components that only support closed proprietary protocols. Applications requiring low latency communications can be deployed to run locally at the edge of the grid.

\subsection{Forecasting Applications}

Forecasting Applications were developed by UTSA for the prediction of short term demand and generation from renewable resources. These applications were based on a neural networks, support vector machines, the processing of sky images and the prediction of solar insolation on the PV arrays. 
These applications were deployed on a newly developed model of a weather station and hosted on the edge of the grid. The forecasting applications were integrated into the proposed control hierarchy at the edge of the grid in the validation at NREL ESIF and in the demonstration at JBSA in San Antonio.

\subsection{MGMS}

The Siemens Spectrum Power Microgrid Management System (MGMS) is a product (pre-existing technology) available under license from Siemens that was integrated with the OpenFMB as part of this project. It is a software solution for optimal microgrid management and control that is based on the Siemens Spectrum Power 7 SCADA/EMS platform. The Siemens MGMS includes advanced functions such as seven-day load and generation forecasting, resource optimization, load shed, seamless planned transition to and from island mode, and market participation applications. The MGMS platform can be applied to all microgrids, whether they are grid connected or islanded, and whether the microgrid is a participant in an electricity market or not. MGMS can be deployed with implementation of a new microgrid or as an enhancement in an existing microgrid.

\subsection{Validation at NREL}

The project team, along with NREL staff validated the concepts of this project at the NREL ESIF in Golden Colorado. The project focused on three major areas; validation of management of a microgrid, validation of an implementation of the OpenFMB Reference Architecture, and validation of innovative methods of forecasting power production from solar PV resources as well as microgrid loads. A set of tests was conducted to validate that the Siemens MGMS can be integrated with the OpenFMB and used to monitor and control a microgrid. The facilities of ESIF were used to create a simulation of a microgrid including typical behavior of loads and renewable resources at a MW scale as shown in Figure 3. 


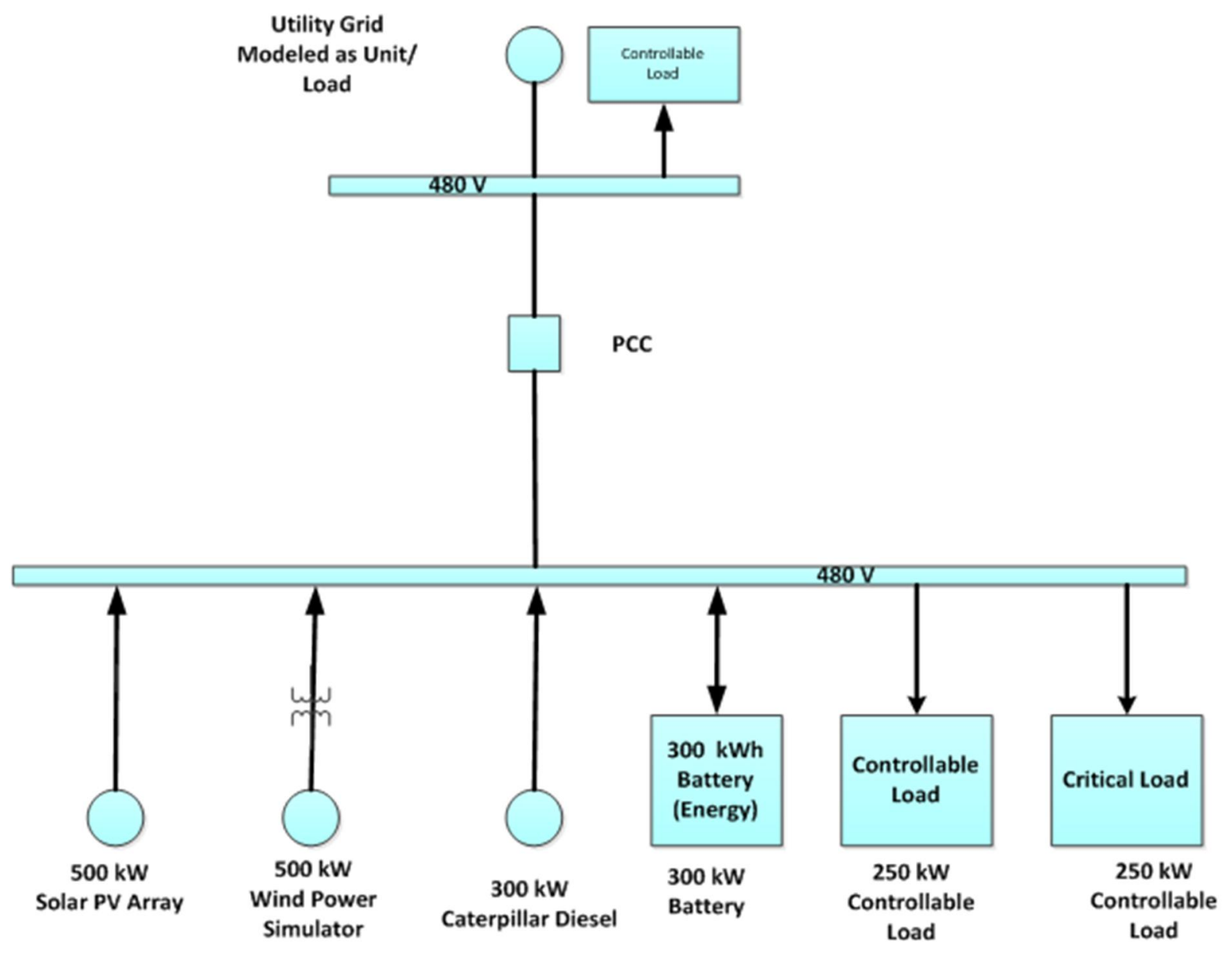

Figure 3: Microgrid with Renewables tested at NREL ESIF

The test results showed that the MGMS can successfully optimize the resources of a microgrid to give a day ahead schedule and to adaptively update that schedule during the operating day. The ability of the MGMS to provide and anticipatory schedules and mandatory dispatch instructions to the controllable resources of the microgrid was validated.

Another set of tests validated the concepts of the OpenFMB architecture. Particular attention was placed on validating the ability of the OpenFMB to support a Monitoring and Control System on a distributed platform that can be hosted at the edge of a distribution grid. Similar attention was also focused on validating the ability of the OpenFMB to allow disparate intelligent devices with different native protocols to interoperate using adapters to the OpenFMB. The ability of the OpenFMB to host different transport protocols (MQTT and DDS) at the middleware level was also included in the test. Figure 4 summaries the OpenFMB implementation tested at NREL. 


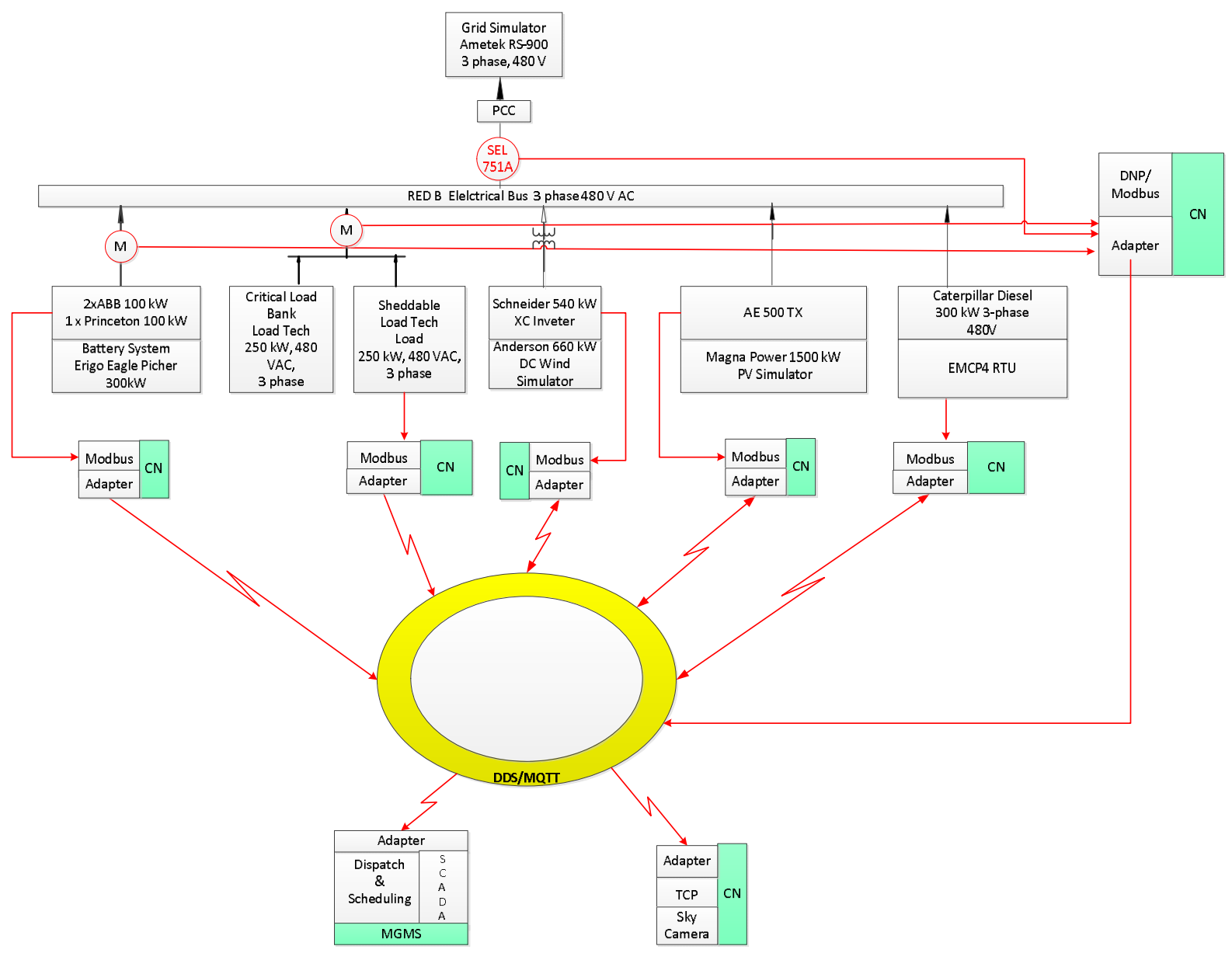

Figure 4: Implementation of OpenFMB tested at NREL ESIF

Forecasting applications were also validated at NREL ESIF. These tests validated the concept of acquiring forecast input data locally and forecasting load and solar PV generation locally. The ability of the OpenFMB architecture to host forecasting applications on a distributed platform was also tested. The accuracy of the forecast applications was also measured with various standard error statics.

Validation testing at the NREL ESIF was followed by a demonstration to NREL and the Industry on May 24, 2016.

\subsection{Demonstration at Duke}

Duke Energy has set up a microgrid at its Training Center in Mount Holly, NC. A microgrid was established in the training center that includes the laboratory workshop facilities as the load of the microgrid. The microgrid resources included a $100 \mathrm{~kW}$ solar PV array and associated inverters that are connected to the $480 \mathrm{~V}$ distribution system. A $250 \mathrm{kWh}$ battery energy storage system (BESS) and a 500 $\mathrm{kW}$ controllable load bank made up the remaining microgrid resources. 
The Mt Holly microgrid operates under an agreement with Duke Energy that requires no reverse power flow from the microgrid into the feeder to which it is connected (Feeder 1201 from the Rankin substation.) Duke has developed a real time control system that varies the consumption of the load bank and the charging/discharging of the BESS to ensure that there is no flow from the microgrid back into the utility feeder. The Mt Holly microgrid also has a protection and control system provided by Duke which supports seamless, unplanned transition from grid connected to islanded operations, and planned reconnection from islanded to grid connected operations.

Using an implementation of the OpenFMB Reference Architecture, the Siemens MGMS was integrated with the real time control application developed by Duke. The Siemens MGMS included load and solar PV generation forecasting applications that are based on a similar day/pattern matching algorithm and an optimization function that produces advisory day-ahead schedules for the next 24-36 hours for the interchange of the microgrid with the feeder at the point of common coupling, and for the desired state of charge of the BESS. The MGMS also produces mandatory dispatch instructions for the next 5-minute dispatch interval in the form of a desired state of charge for the battery at the end of the interval.

In the implementation at Mt Holly, optimal schedules were produced by the MGMS which respected the constraint of no reverse power flow to the utility grid and were published as advisory schedules. A dispatch signal was sent to the BESS as the desired state of charge for the end of the next 5-minute interval. This signal was integrated with the Duke developed real time control system that varies the consumption of the load bank and the charging/discharging of the BESS to ensure that there is no flow from the microgrid back into the utility feeder.

These applications were hosted on distributed platforms and were integrated with an implementation of the OpenFMB Reference Architecture as shown in Figure 5. This implementation was successfully site tested and demonstrated to NREL and the industry on June 28, 2016. 


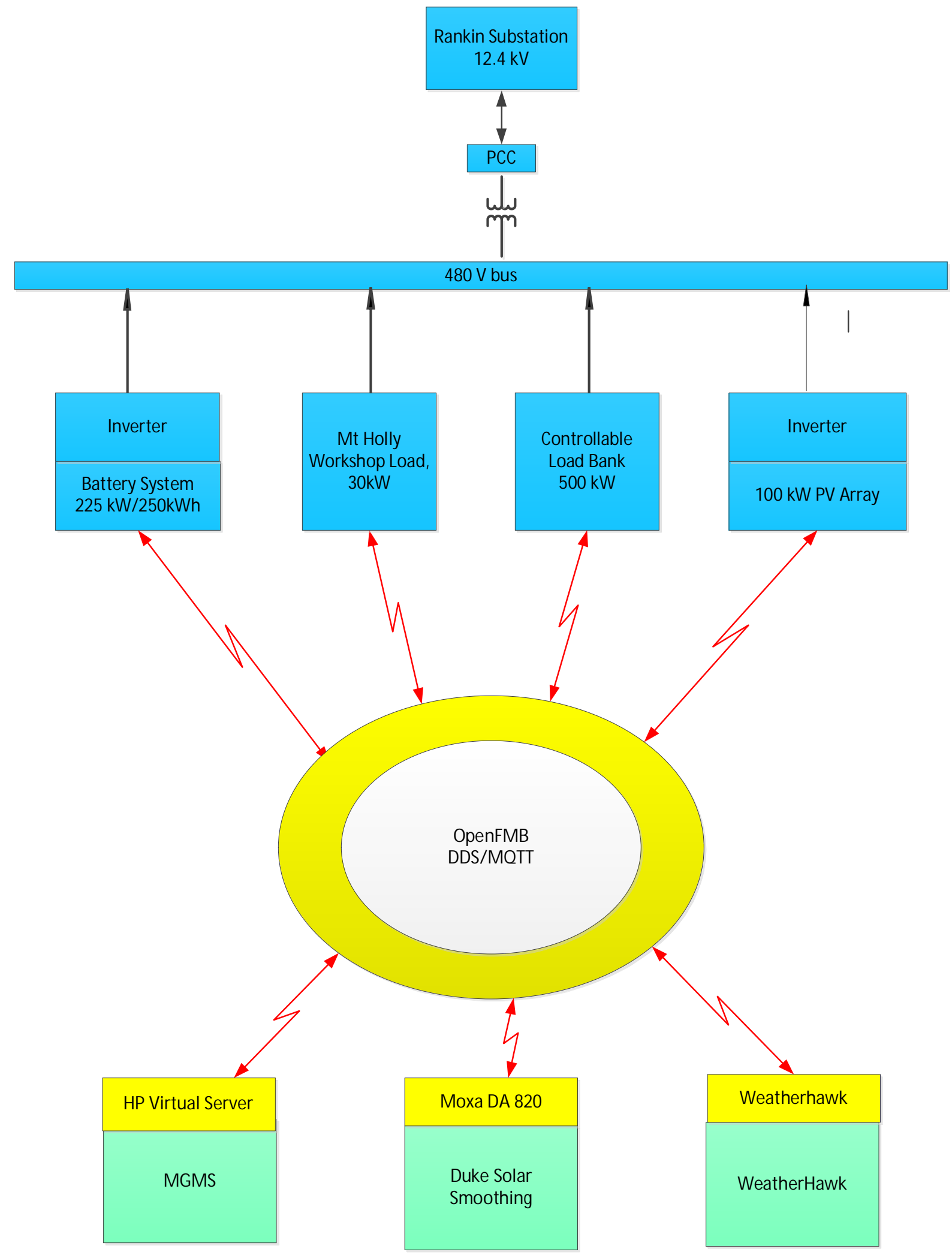

Figure 5: Implementation of OpenFMB tested at Mt Holly Training Center 


\subsection{Demo at CPS}

CPS Energy of San Antonio TX (CPS Energy) has developed a microgrid at the Joint Base San Antonio which is a military facility for the US Army, Navy, Airforce, Marines and Coast Guard. The microgrid includes a solar PV array of $20 \mathrm{~kW}$ and a $75 \mathrm{~kW} / 48 \mathrm{kWh}$ battery located in close proximity to the JBSA library that comprises the majority of the microgrid load which varies in the range 40-68 $\mathrm{kW}$ depending on the time of day and seasonal cycles.

An implementation of the OpenFMB architecture was set up at the JBSA that integrated the Siemens MGMS, the Battery Management System, and the set of Forecasting Applications developed by UTSA. The implementation was successfully site tested and demonstrated to NREL and the industry on September 27, 2016. 


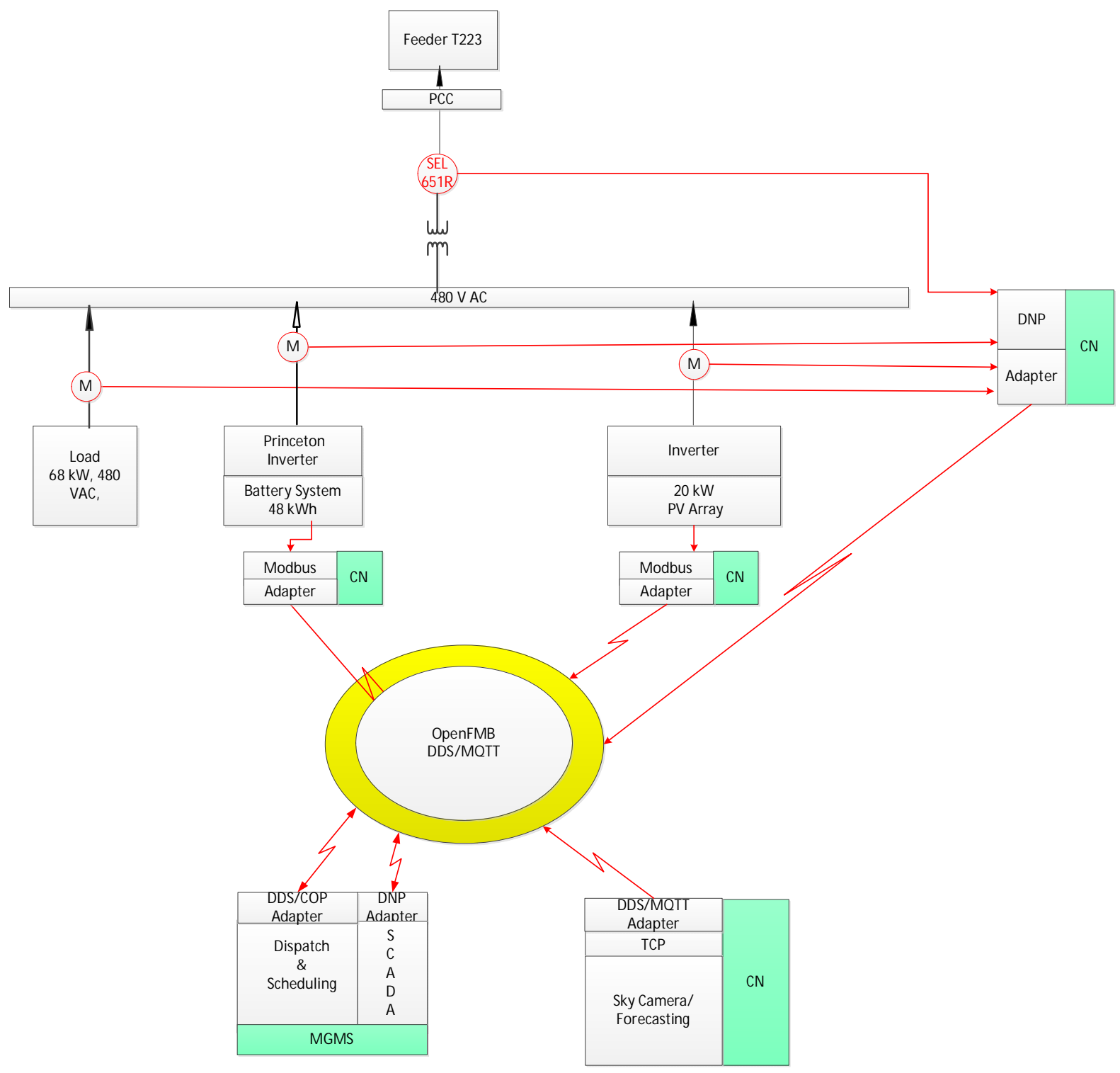

Figure 6: Implementation of OpenFMB tested at JBSA

\subsection{Standards}

The Open Field Message Bus is based on commonly available "open" standards to achieve its mission of enabling interoperability of new equipment with older equipment that may support only proprietary, closed protocols. A key to the OpenFMB is the publish/subscribe design pattern using the protocols Data Distribution Services (DDS), MQTT (Message Queuing Telemetry Transport), and the Advanced Message Queuing Protocol (AMQP). The Global Data Space used by OpenFMB is based on the IEC CIM UML model. The results of the project are available either through NREL or through the SGIP under an open source license. The OpenFMB Architectural Framework is available from NAESB as RMQ.26. 


\subsection{Lessons Learned}

During the courses of the project, lessons were learned that should prove valuable to future implantations of the OpenFMB. These lessons as well as suggestions for further research are detailed in Section 11 of the main report.

\subsection{Cost Benefit Analysis}

The project includes an assessment of the costs and the benefits of a microgrid. The findings of this part of the project were that the costs and benefits vary depending on several key factors: geography (urban vs rural), climate (number of sunny days), the design of the microgrid, whether the microgrid participates in a regional electricity market and the tariff structure of the utility to which the microgrid is connected. The cost benefit analysis includes a base case for providing reliable power, and several cases that vary the parameters mentioned above. The cost benefit report shows which parameters are the most influential in determining whether a microgrid is beneficial from a net present value and cost/benefit point of view. 


\section{Introduction}

OMNETRIC Corp., Duke Energy, CPS Energy, and the University of Texas at San Antonio (UTSA) created a project team to execute the project NREL Integrate RCS-4-42326, Topic Area 3: Integrated Systems: "OpenFMB Reference Architecture Demonstration." The project included development and demonstration of concepts that will enable the electric utility grid to host larger penetrations of renewable resources. The project concept calls for aggregation of renewable resources and loads into microgrids and the control of these microgrids with an implementation of the OpenFMB Reference Architecture.

The OpenFMB Reference Architecture will enable the grid to effectively support large-scale complex operations, such as with distribution systems at electric utilities, which allow for an integration and wider penetration of renewable, clean energy resources. Management of distribution systems with large penetrations of renewable resources is impacted by two major issues: (1) the lack of standardization and interoperability between the vast array of equipment in operation and on the market, most of which use different and proprietary means of communication and (2) the magnitude of the network and the information it generates and consumes.

The OpenFMB is an open-source platform with a reference architecture that is scalable, reliable and secure and that accommodates highly diverse types and brands of equipment. This solution provides an integrated and interoperable working system that provides "real time" risk management and cost control. Scalability is achieved through the deployment of computing resources and intelligence at various levels (i.e. control center versus device level) throughout the grid. The OpenFMB strives to optimize locations for key operational functionality, including locations nearest to the renewable resources that are managed "at the edge of the grid".

This report includes a description of the validation of the project concepts in a laboratory setting at the National Renewable Energy Laboratory (NREL), Energy Systems Integration Facility (ESIF), and in field demonstrations at both Duke Energy and CPS Energy. An assessment was made of both technical and economic costs/benefits of the project concept. 


\section{Microgrid as an element of a control hierarchy}

The electric grid in North America is a multi-level hierarchical system with a (possible) Independent System Operator/Regional Transmission Operator (ISO/RTO) level managed by an Energy Management System (EMS) and an Energy Market Management System (EMM); a utility level managed by an EMS; and one or more levels of utility Distribution Management System (DMS) systems (Figure 1). Recent innovations have begun to create a microgrid level that consists of small subsets of resources and grid assets including load, renewable resources, conventional resources, demand response, and energy storage systems within clearly defined boundaries which may be disconnected from the larger grid and operated with local supply. Microgrids may be owned by the utility or by some other party. This project proposes the concept of a Microgrid Management System (MGMS) that manages the operation of the microgrid and cooperates with the next level of the control hierarchy by presenting an aggregated, abstracted view of the microgrid to the utility that can be easily incorporated into the DMS and EMS systems that utilities use to manage the grid today. This approach will allow the grid to operate with a very large number of microgrids, each of which may contain large amounts of variable renewable resources, in a fully integrated and reliable manner.

\subsection{Problem statement}

In the past few years, there has been tremendous growth in the deployment of renewable resources on the power grid in North America. These resources provide electricity from sources that are both clean and renewable at a cost that is increasing competitive to traditional fossil fueled and nuclear resources. While the use of these resources brings very many benefits such as clean air, clean water and the promise of national energy independence, the use of these resources also brings new challenges, especially in the realm of power system operations.

In the absence of significant energy storage capacity on the grid, the production of power from all sources must identically match the demand for power at each instant of the day. When the percentage of power from variable renewable resources becomes a significant percent of total demand, power system operators responsible for balancing total production with total demand are faced with increasingly severe operating problems.

The production of power from the renewable resources that are appearing on the grid today is very closely linked to the weather. The difficulty of forecasting the weather, which is well understood, extends to the difficulty in forecasting the production of renewable resources. The current state of the art in forecasting the power production from renewables (solar PV and wind) are accuracies in the range of 12-25 percent NMAE as shown in Figure 8. In contrast the use of electricity aggregated to the system level, is easier to predict. The state of the art of load forecasting done 24 hours ahead, is about 2-3\% MAPE, as shown in Figure 7. Forecasting the load to be supplied from conventional resources (load minus generation from 
renewable resources) is thus very hard to forecast. This means that even a few hours before the time of operations, there can be considerable uncertainty over what is needed to balance supply and demand.

Adding to the problem of difficulty of forecasting, is the reality of variability of the actual production of power from renewables, as shown in Figure 9. Due to the variability of wind speeds and solar insolation, the actual output of power from renewable resources can vary significantly. Gusts of winds result is variation of power output of wind turbines. The shadows of clouds moving over solar PV arrays result in the variation of power production of the array. This compounds the problem of balancing supply and demand in real time.

These problems can be managed using one or more the methods described below.

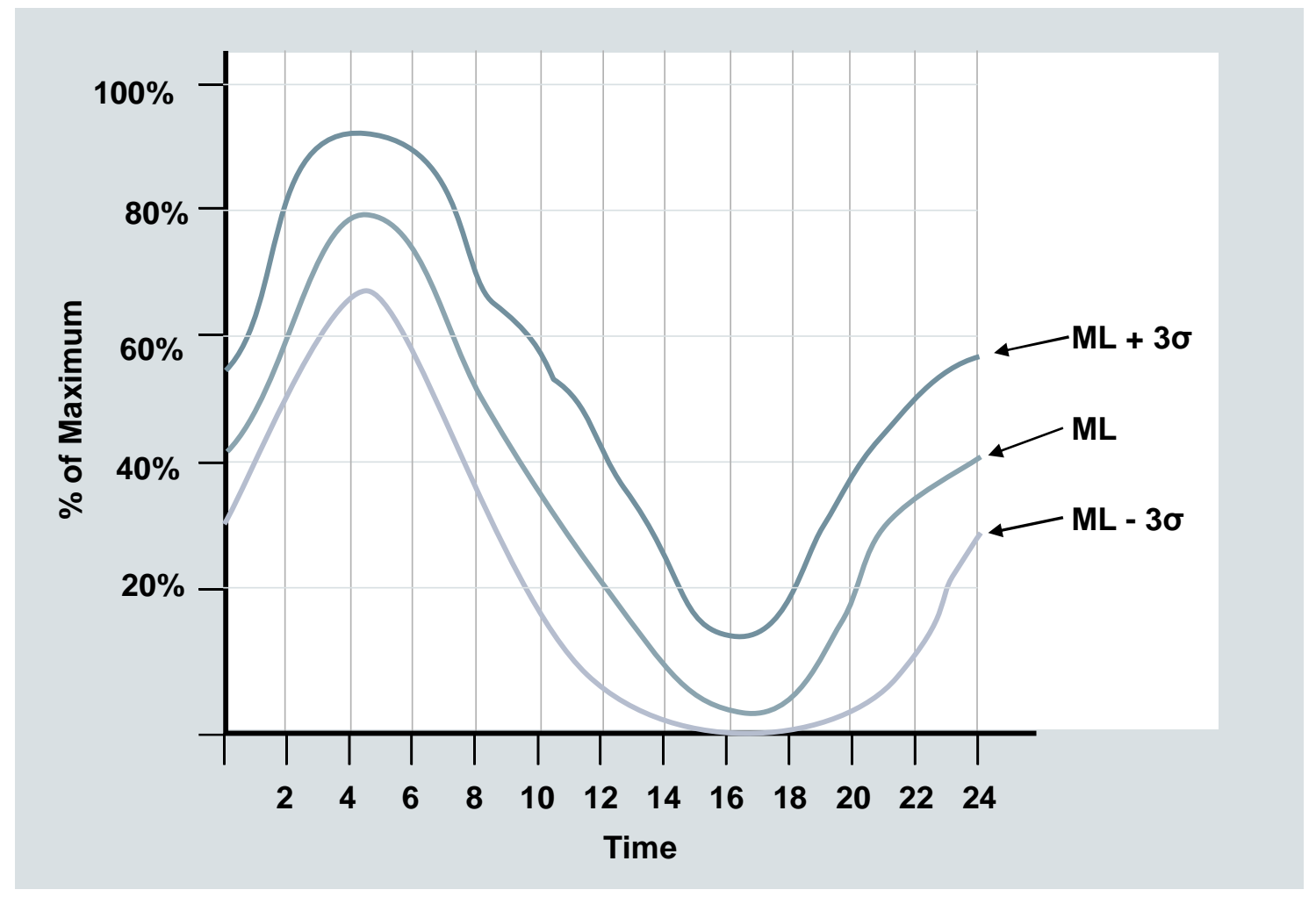

Picture of forecast of demand with cone of forecast uncertainty

Figure 7 Short Term Load Forecast 


\section{Figure 1: Probabilistic Display Concept}

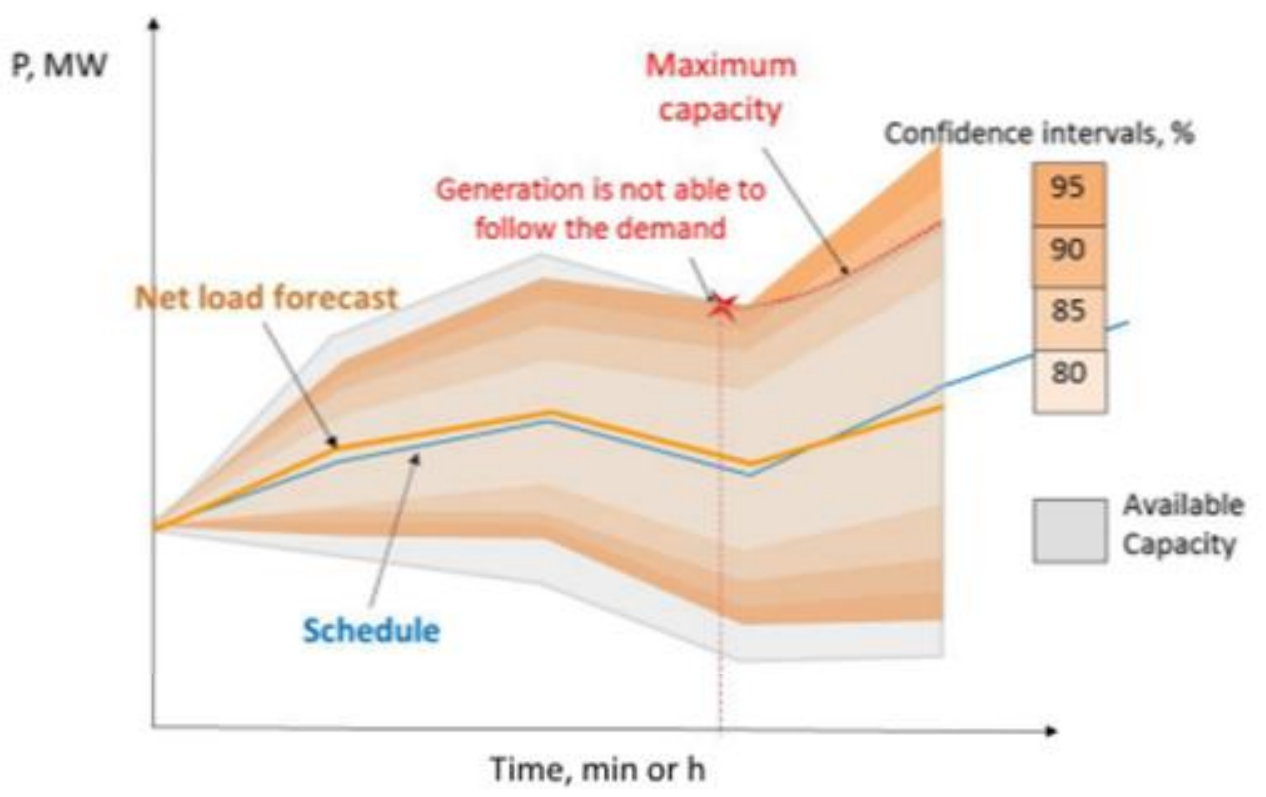

Figure 8 Picture of forecast of renewables with larger cone of uncertainty-will be replaced with better graphic

Figure 8: Short Term Solar PV Generation Forecast

\section{April 15}

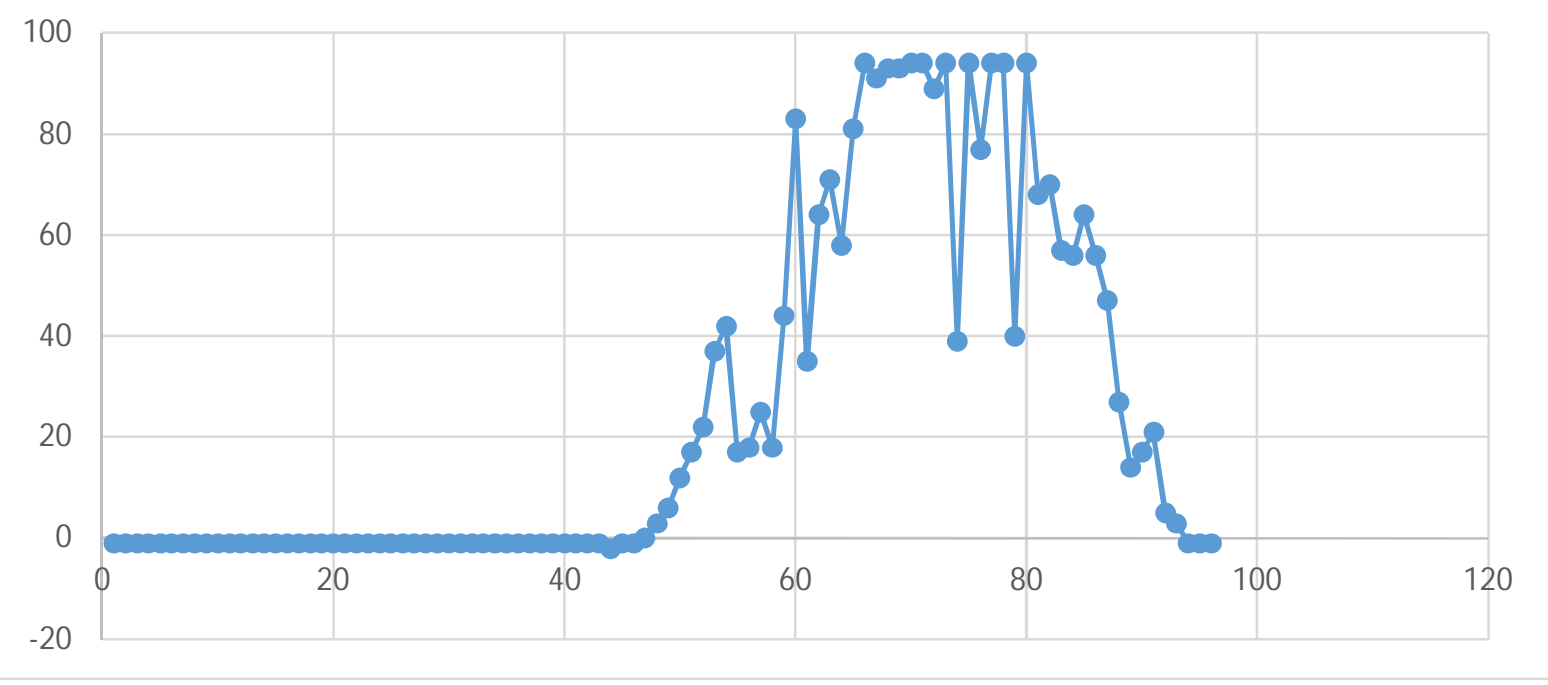

Figure 9: Actual Solar PV Generation 


\subsection{Possible solutions}

The problem of balancing supply and demand can be addressed in a number of ways:

- Adjustment of production from renewables

- Deployment of flexible loads

- Use of energy storage systems

- Use of reserves produced by energy systems based on different technologies

\subsubsection{Adjustment of Production from Renewables}

In times of over-generation, it is possible to reduce or curtail the production of power from renewable resources. Wind energy systems may be "dispatched down" by sending a setpoint to the wind energy control system that controls the pitch of the turbine blades ("feathering") or that takes the turbine off-line. In a similar fashion, a solar PV system may be dispatched down by sending a setpoint to a smart inverter instructing it to produce less power than it is currently capable of given the solar insolation.

In times of under-generation, there may be the option of increasing the steady state power output of a wind energy or solar PV system up to its current operating limit if it has been previously dispatched to operate below its current operating capability.

\subsubsection{Deployment of Flexible Loads}

Flexible loads may also be used to balance variable power production from renewable resources.

Demand response programs and loads that participate in wholesale markets can adjust consumption when there is under-generation from renewables. Some forms of flexible load (e.g. pumping water for irrigation in agriculture) can be increased to balance the system during periods of over-generation.

\subsubsection{Energy Storage Systems}

Energy Storage Systems may be used to balance variable power production with demand requirements by adding to stored energy in times of over-generation and releasing stored energy in times of undergeneration. Early forms of stored energy were pumped hydro systems. This form of energy storage system requires both suitable geographic terrain and available water systems that are used by electric motor generator sets to pump water to a higher elevation or release water to turn a water turbine and generate electricity

Recently there have been developments in the area of batteries of various chemistries that can be charged and discharged as electrical energy. 


\subsubsection{Reserves Based on Different Technologies}

Balancing of variable renewable generation with required demand may also be done with flexible generation based on a different technology. Natural gas fueled combined cycle plants with short startup/shutdown times and very fast ramp rates may be used to correct for over-generation/undergeneration and ramping of variable resources. Hydro resources may also be used for this purpose, when available on the grid.

The power system operator may include these resources either on a standby basis, or may require the resource to be synchronized on the grid depending on the time frame in which the reserves may be called on to balance variable renewable generation.

\subsubsection{Increasing Penetration of Renewable Resources Exacerbate the Problem of Balancing}

When the amount of renewables on the grid was a small percentage of the total system load, it was relatively easy for operators to manage the variability of their production. This was often accomplished by simply maintaining system reserves at already established levels required for reliability (regulating reserves, spinning reserves, ready reserves, quick start reserves, etc.). However, as the amount of renewables increases, new approaches are required. As the production from renewable resources increases to 10 percent, 20 percent or more of system load, the effects of the variability of the resources begin to have larger effects on system operations, in terms of high and low frequency excursions, unpredicted flows on lines, and over/undervoltages at key buses on the system. At even higher levels of penetration (say 50 percent) it is no longer economic or even feasible to carry enough reserves on-line for balancing. A new approach is required.

\subsection{Recommended Solution}

One possible solution is to create a hierarchical control scheme made up of a set of microgrids. Each microgrid will have responsibility for managing its resources so that its behavior is predictable. The following sections elaborate on these concepts.

\subsubsection{Microgrids}

A microgrid can be considered to be a set of renewable resources, conventional resources, flexible loads, critical loads, and energy storage within clearly defined boundaries which can operate while connected to a utility grid, or can operate while disconnected from the utility. The resources of the microgrid may be utility owned or may be owned by a third party. A microgrid may be as small as a facility with a solar PV array, a load, and an energy storage system (battery) or as large as a campus with several loads, solar PV arrays, CHP plants, diesel generators, and energy storage. 
A microgrid is connected to the utility grid at a point of common coupling (PCC). Typically this is a load break switch which may be used to separate the microgrid from the utility grid for stand-alone operations, or to re-synchronize the microgrid with the utility grid (possibly with an auto synchronization control systems). It is also possible to have several PCCs for a microgrid, but this requires more complex relaying schemes for isolation/resynchronization.

In the recommended solution, a microgrid is controlled by a microgrid management system (MGMS). This MGMS has responsibility for transition of the microgrid from grid connected to islanded operations and from islanded operations to grid connected operations. The MGMS also has the responsibility for managing and optimizing the operations of the microgrid.

\subsubsection{Predictable behavior of microgrids}

In the recommended solution, each microgrid forecasts its behavior, and controls its resources to follow its predictions. The MGMS includes a state of the art forecasting application which forecasts the load demand of the microgrid, and the production of power from renewable resources. Using these forecasts as inputs the MGMS optimizes the resources of the microgrid according to a selected optimization objective. For example if the selected objective is to use the most economic resources in a situation where the utility grid has time-of-use pricing, the optimization may select the use of stored and renewable resources during times of high utility prices and use utility resources during the times when utility prices are lower.

The optimization horizon for the MGMS can range from 6 to 30 hours. It typically optimizes for the remainder of the operating day but at the end of the operating day, the horizon extends to the following operating day. The optimization is typically done in intervals of 15 minutes. The results of the optimization are the operating plan for the time horizon. This includes schedules for:

- Forecast generation from renewable resources (input data)

- Predicted schedules for generation from renewable resources (may include curtailments)

- Forecast demand for microgrid (input data)

- Predicted schedule for flexible loads (may include curtailments)

- Charge/Discharge schedules for energy storage systems

- Import/export of energy with the utility microgrid

The import/export schedules of the microgrid are reported to the utility as the forecast behavior of the microgrid in a day-ahead schedule.

During the operating day the MGMS both re-optimizes and dispatches the resources in the microgrid. Dispatch signals are sent to the microgrid resources for the next operating interval which is typically a 5minute interval. The resources of the microgrid adjust their outputs as a result of these dispatch instructions. 
The MGMS also re-optimizes the operations of the microgrid for the remainder of the operating day, but with the import/export schedules held at the levels reported to the utility in the day ahead schedule. In the case of infeasibility of solution, the MGMS reports an updated import/export schedule to the utility. This is done on a rolling basis, every 15 minutes as shown in Figure 4.

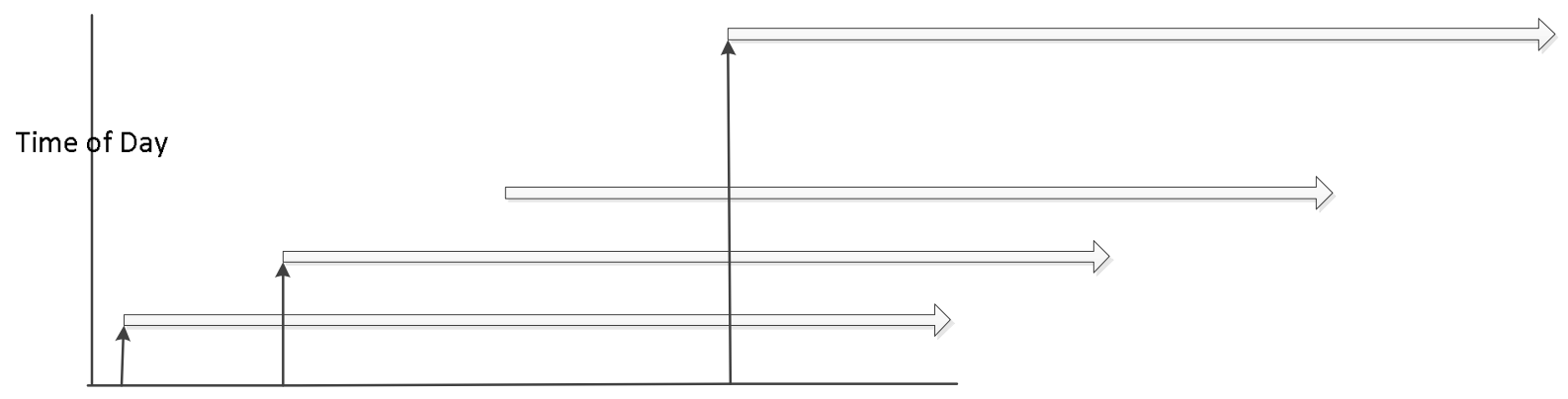

Optimization Horizon

Figure 10 that shows on the y axis the time of run and the $y$ axis the remainder of the operating day.

Figure 10: Optimization over a rolling horizon

\subsubsection{Response of Utility System Operator}

The important point of this rolling horizon is that the MGMS detects any infeasibility of the microgrid to follow the committed day-ahead schedule with a lead time that may allow the utility system operator to take corrective action. For example, in a situation where there is a dramatic decrease in the forecast for solar insolation, the MGMS will may send the utility operator a revised schedule showing increased imports from the utility grid over the original forecast.

The system operator (aided by automated tools) may conclude that the error of this microgrid may be offset by an error of another microgrid showing an increase in exports (destructive interference). On the other hand the system operator may conclude that the error for increased imports is systemic (constructive interference) as shown in Figure 11. If the system operator is aware of a developing operating problem before it actually occurs (situation awareness), the operator may have the lead time required to start (or ramp) a unit that uses another technology (hydro or natural gas) or may deploy demand response elsewhere in the system to the balance the power supplied by renewables with the load demands. 

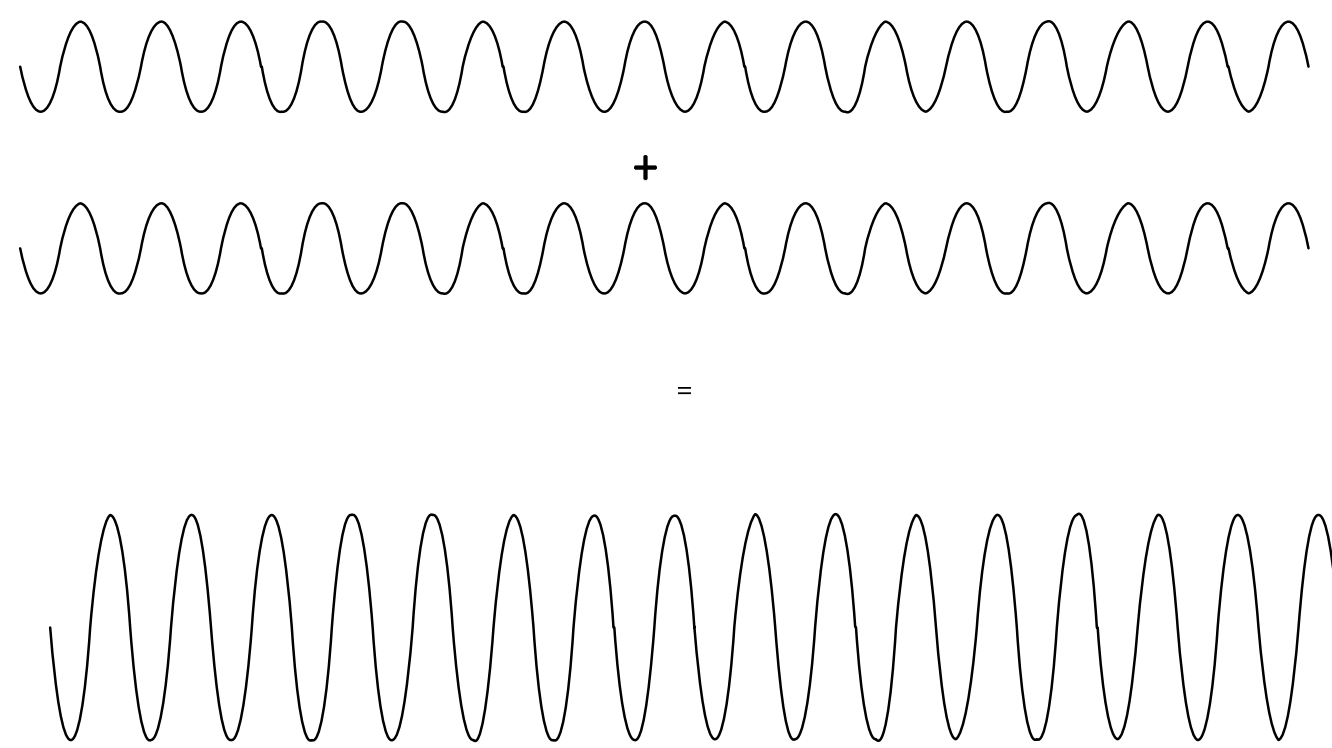

Constructive Interference

or

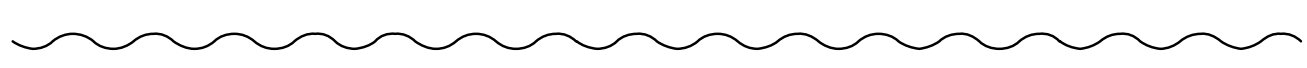

Destructive Interference

Figure 11 destructive and constructive interference will be replaced with better graphic

Figure 11: Constructive and Destructive Interference

In another example, there may be an excess of renewable power over the day ahead forecasts. With enough lead time the system operator may be able to take corrective action such as shutting down a unit, scheduling flexible loads, or arranging a sale of power to another part of the interconnected grid, without having to call for curtailment of renewable energy across the system.

\subsection{Scalability of recommended solution}

The recommended solution is scalable. A large utility may have too many renewable resources to manage centrally. For example a utility with a million customers may have, in a scenario of high penetration, hundreds of thousands of solar PV arrays on its distribution network and hundreds of thousands of flexible loads, and storage systems. Optimization problems of this scale are very impractical to solve in real time with central computer resources. Challenges include:

- Collecting and verifying model parameter data

- Communication of run time data to central optimizer

- Validation of run time data by central optimizer

- Time to solve large central problem

- Time to validate of solution 
- Communication of solution to controllers of end resources

- Actuation of solution as setpoints to devices

The recommended solution partitions the grid into a number of smaller grids, each of which does local optimization and collaborates with the utility control system. The recommended solution can be scaled to 1000's of microgrids and thus enables the grid to accommodate high penetrations of renewable power on the grid (over 50 percent).

Another advantage is that the recommended solution hides the complexity of microgrid resources from the utility system operator, since the entire microgrid may be represented as a single load/generator. The solution can be scaled to include ISO/RTO as an additional higher level controller as shown in Figure 12. The hierarchical control scheme additionally meets the challenges described above.

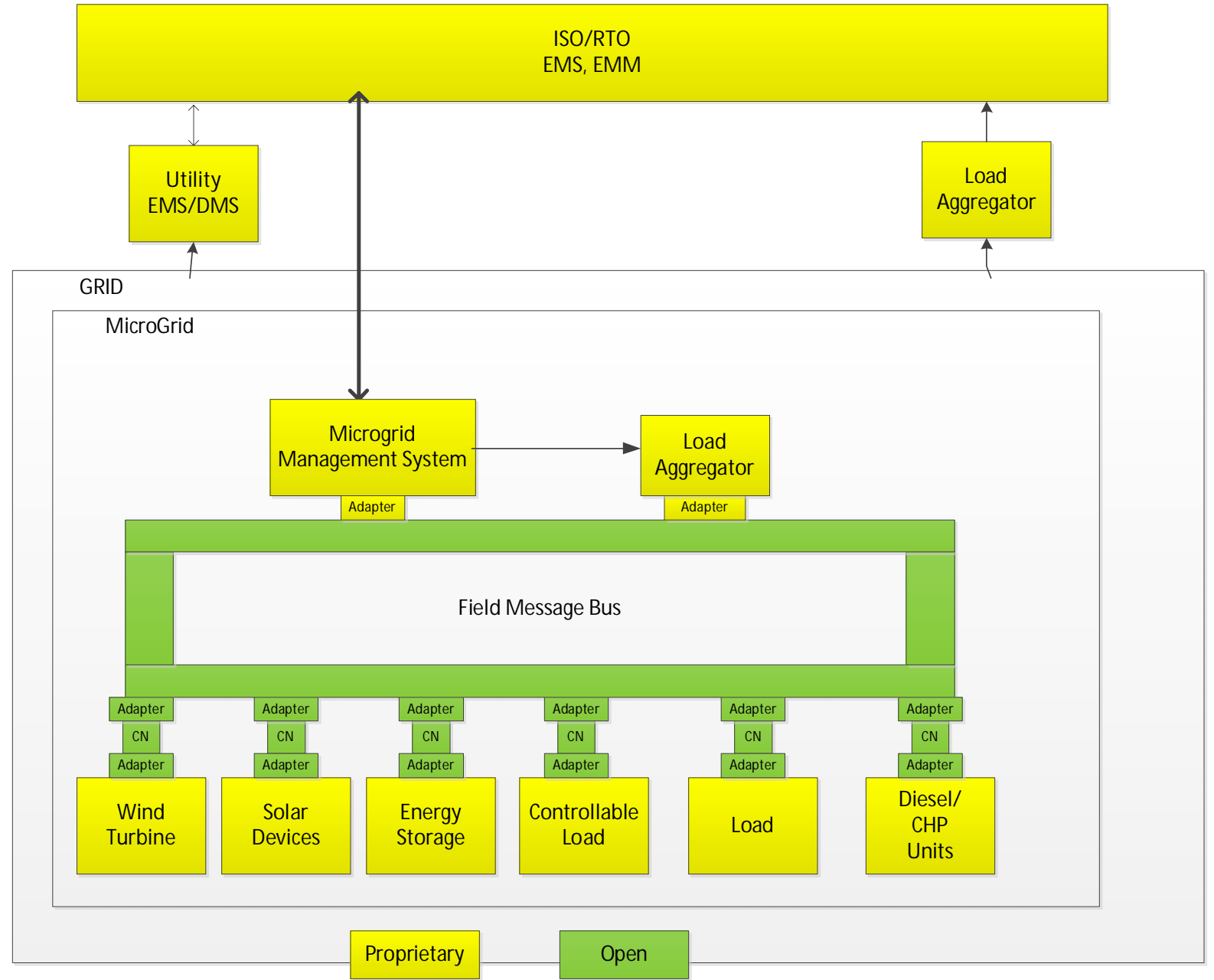

Figure 12: Hierarchical Control System 


\subsection{Resiliency}

Aggregation of renewables into managed microgrids that behave in a predictable manner enables the grid to support much larger penetrations of renewable resources as previously discussed. And there is also the added benefit of increased reliability. Microgrids that have been designed to support unplanned transition to islanded operations will continue to supply power to loads that have been pre-designated as critical loads. In the event of loss of external supply from the grid, the now islanded microgrid will continue to supply local critical loads with generation that is on-site. The designation of which microgrid loads are critical will have to be done at the time of design and implementation of the microgrid management system. The use of a microgrid to provide highly reliable power to critical loads is an additional benefit to the benefit of enabling the grid to support more renewable energy resources. This value has been quantified in one of the use cases studied in this project. Please see Appendix A for a summary of this cost benefit analysis.

\subsection{Requirements for Hierarchical Control}

In order to realize the control scheme described above, the following are areas that required technical improvements that were addressed as part of this project.

\subsubsection{Improved communications, control, and Interoperability}

The recommended solution of aggregation of renewables into microgrids that behave in a predictable way, requires advances in the areas of communications and control. There are many vendors of renewable resources that support only proprietary protocols, and a vast array of Intelligent End Devices (IEDs) that also support different connectivity, protocols, and semantic models for communications but that do not easily interoperate with each other. This project included an in depth evaluation of the newly developed integration framework, The Open Field Message Bus (OpenFMB), ability to integrate disparate resources within a microgrid. The technical approach is discussed in Section 4.

\subsubsection{Improved forecasting}

Improvements are required in both the areas of load forecasting and generation forecasting. Improvements in the areas of load and generation forecasting are described in Section 5.

\subsubsection{Microgrid Management System}

A Microgrid Management System is required to monitor and control the resources inside the microgrid, and to establish and communicate its behavior (import/export schedules) to the utility grid management system, typically a Distribution Management System. There are several vendors of MGMS systems on the market. This project included the Siemens Spectrum Power 7 MGMS and its capability to determine 
optimum schedules over an operating horizon of a few minutes to a few days. This MGMS is further described in Section 6. 


\section{Open FMB}

\subsection{Background}

This project included an in depth evaluation of the newly developed Open Field Message Bus (OpenFMB). The OpenFMB was initiated by Duke Energy, initially as a Distributed Intelligence Reference Architecture [1], and then rebranded as the OpenFMB. The OpenFMB was adopted by the Smart Grid Interoperability Panel (SGIP) [2] and was documented by the North American Energy Standards Board (NAESB) Retail Market Quadrant model business practice [3]. The following paragraphs briefly describe the fundamentals of the OpenFMB.

The OpenFMB is an Architectural Framework which may be used to integrate distributed intelligent nodes to exchange data and interoperate with each other to support a large variety of electric utility operations. The OpenFMB extends the concepts of integration of enterprise applications using an enterprise service bus (ESB) to an integration framework that may be applied in the field at the edge of the utility distribution grid to integrate grid edge nodes that acquire and process data locally for control and reporting. It applies concepts from the Industrial Internet Consortium [4] to the electric utility smart grid.

Key characteristics of the OpenFMB are: its publish/subscribe integration pattern, a global data space based on a standard semantic model, and its ability to support distributed logic as well as centralized logic. These characteristics enable peer to peer communications and provide a standardized way for grid edge applications to communicate and access data. The use of the standard semantic model enables the development of adapters to gain access to power system data from legacy components that only support proprietary closed protocols. Applications requiring low latency communications can be deployed to run locally at the edge of the grid.

\subsection{Communications Node}

Key enablers of the OpenFMB are the newly available smart devices from the field of the Internet of Things, which include a variety of computing and connectivity features. Duke Energy has envisioned an idealized Communication Node (CN), Figure 13, with the key functionality required to support the OpenFMB. Such a communication node will support connectivity for measurement, control and communications using a wide variety of standard interfaces and protocols:

- $\quad$ serial, (RS 232/RS-485),

- Ethernet (8P8C/RJ45)

- Ethernet Fiber Optic)

- Ethernet (4G LTE) 
- Wi-Fi (IEEE $802.11 \mathrm{a} / \mathrm{b} / \mathrm{g} / \mathrm{n})$

- GPS (precise time)

- Power Line Carrier

- Radio Frequency ISM

- Bluetooth

- I/O Metrology

The Communication Node will include Internet Protocol Router capabilities, protocol translation, and a platform for distributed computing. In summary, an idealized Communication Node will support the communications suites shown in Table 1, with relationships to OSI Reference Model [5].

The platform for distributed computing will include a general purpose CPU, addressable memory, standard operating systems, cyber security, and an open API which will enable $3^{\text {rd }}$ party applications to run on the platform and communicate with other CNs.

The idealized Communication node will be deployable in various form factors, also from Duke [1]:

- Hardened for mounting in an existing outdoor enclosure (IEEE 1613)

- Ruggedized for mounting outdoors (NEMA 4+)

- Rack-mounted for inside a substation control house (IEC 61850-3 or IEEE 1613)

- Integrated (DIP requirements) for new endpoint grid device.

Another option for deployment of the $\mathrm{CN}$ is as a virtual node (on a remote server rack or in the "cloud"), but with local interconnection interfaces that are deployed in one of the form factors above.

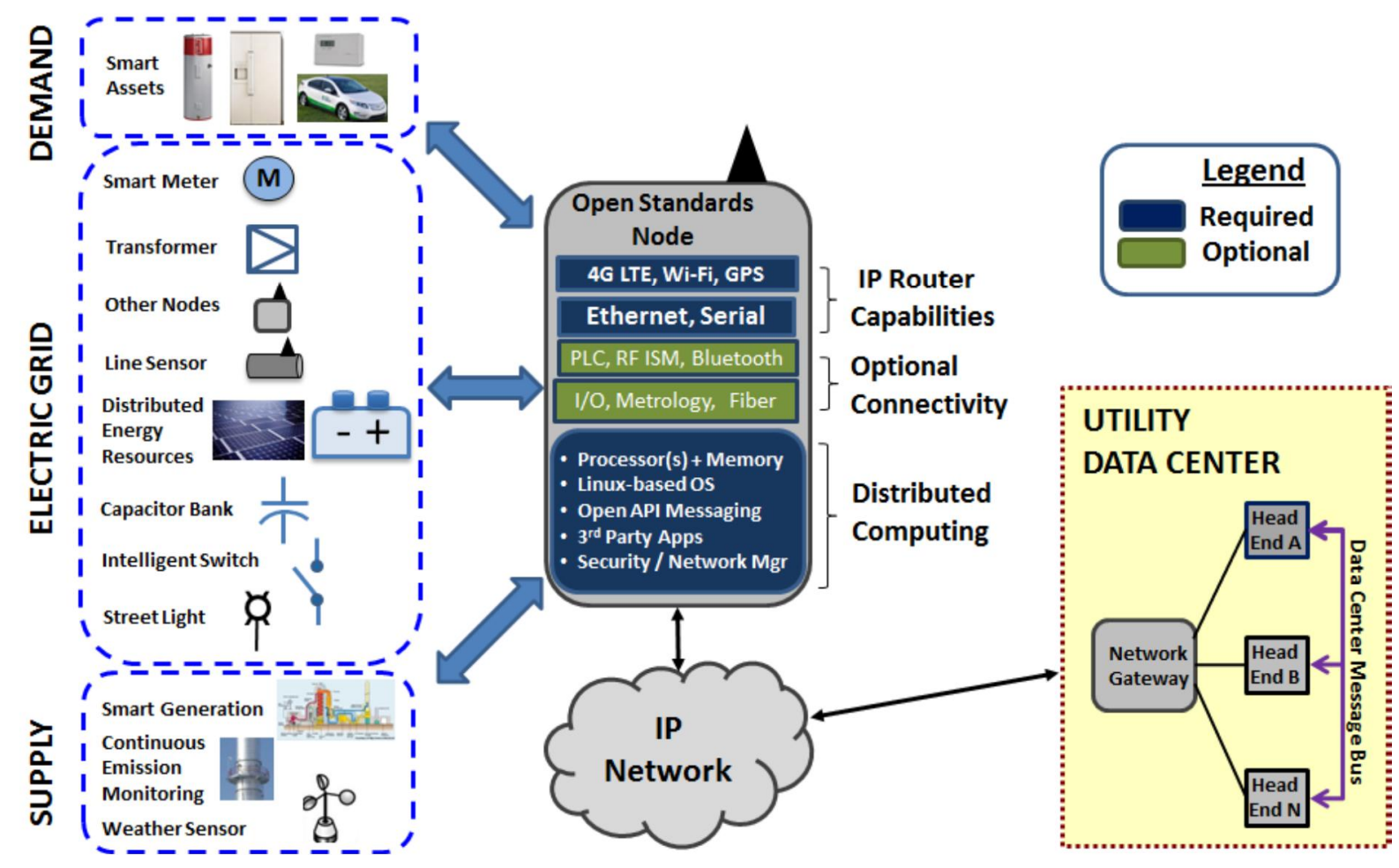


Figure 13: Communication Node

Table 1: OSI Model and CN Internet Protocol (TCP/IP) suite and other relevant protocols

\begin{tabular}{|c|c|c|c|}
\hline Layer & OSI Model & TCP/IP & Protocols \\
\hline 7 & Application & \multirow{3}{*}{ Application } & \multirow{3}{*}{$\begin{array}{l}\text { HTTP, Telnet, SMTP, DHCP, DNS, TLS, SSH, } \\
\text { SSL, SNMP, XML, DNP, C12, REST, MQTT, } \\
\text { DDS, AMQP, CoAP, Modbus, ICCP, OPC }\end{array}$} \\
\hline 6 & Presentation & & \\
\hline 5 & Session & & \\
\hline 4 & Transport & Transport & TCP, UDP, DCCP \\
\hline 3 & Network & Internet & IPsec, IPv4, IPv6 \\
\hline 2 & Data Link & \multirow{2}{*}{$\begin{array}{l}\text { Network } \\
\text { Interface }\end{array}$} & \multirow{2}{*}{$\begin{array}{l}\text { IEEE 802.3, 802.11, 802.15.4, 802.16, Bluetooth, } \\
\text { MAC, PDCP, RLC }\end{array}$} \\
\hline 1 & Physical & & \\
\hline
\end{tabular}

In the OpenFMB framework, the communications node is used to host functionality that is most effectively realized at the edge of the grid, including the following:

- data aggregation, filtering, and prioritization of data from several end points

- short term storage of data, audit information and diagnostics

- Routing, bridging gateway to IP networks

- Conversion of serial to IP

- Remote configuration and device provisioning

- An open API to allow $3^{\text {rd }}$ parties to write applications

- Standards based publish-subscribe messaging

- Hosting of adapters from pub/sub message protocols to proprietary protocols used by legacy assets

- Hosting of $3^{\text {rd }}$ party applications

- Support for distributed cyber security functions

- Support for remote configuration, updates, and provisioning

There are several commercially available IEDs, Utility Grade Routers, Industrial Controllers, and Ruggedized PCs available in the market place today that approximate the idealized communications node, with various subsets of the listed functionality. Many of these devices were included in the deployments of the OpenFMB in this project, as described in subsequent chapters.

\subsection{Reference Architecture}

The OpenFMB Framework was balloted and accepted by the North American Energy Standards Board (NAESB) Retail Market Quadrant as a model business practice [3]. This section briefly summarizes the Architectural Framework. The OpenFMB Operational Logical Architecture [3], as shown in Figure 14, consists of three layers: an application layer, an interface layer and a middleware (pub/sub) layer. 


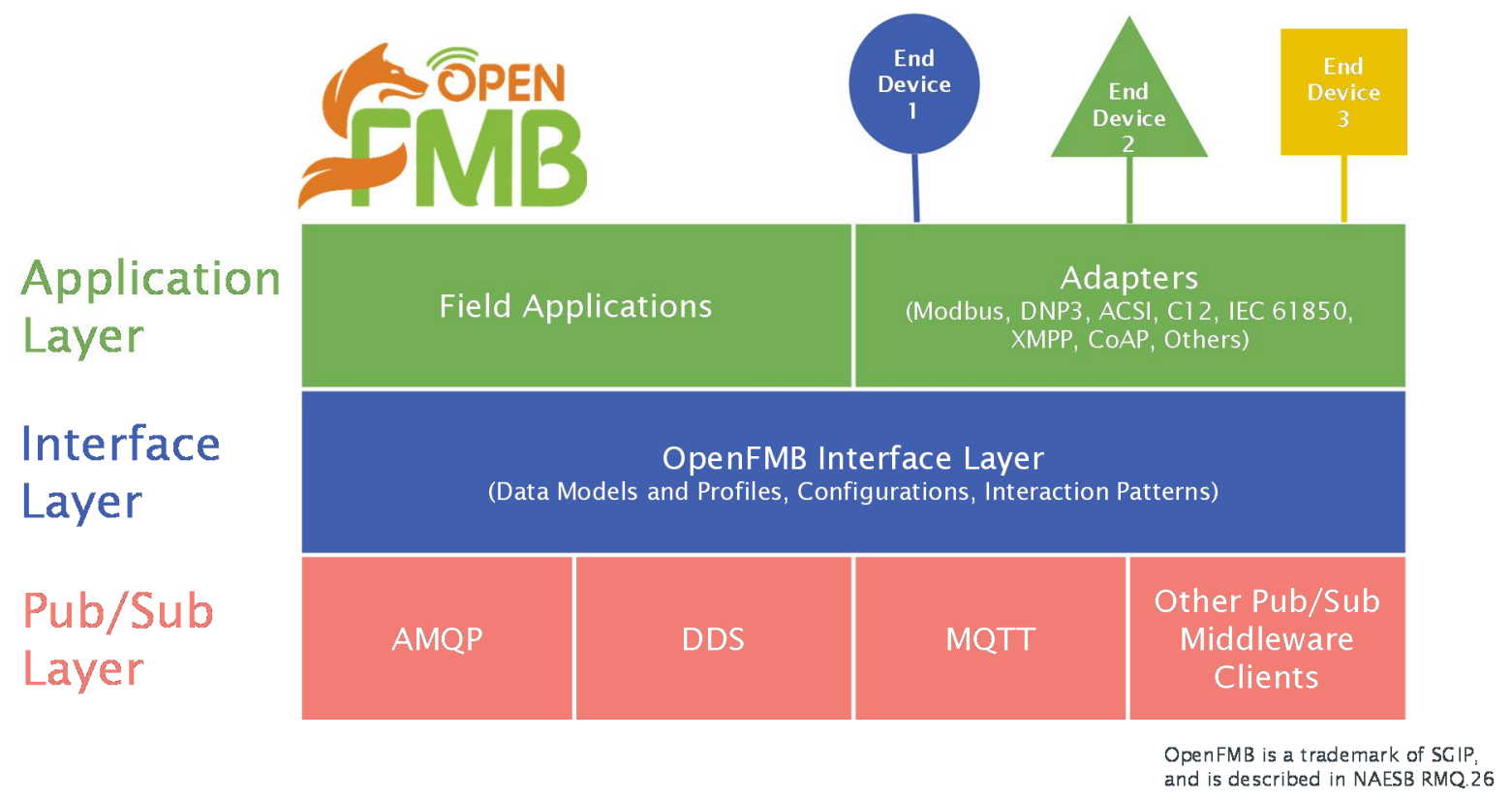

Figure 14: OpenFMB Operational Logical Architecture

OpenFMB applications and adapters exists in the application layer of the framework and run in an OpenFMB Communication Node using the Open API to the other functionality of CN. It is anticipated that the OpenFMB Framework will be used to enable a wide range of new applications that acquire data and issue controls locally with very low latency.

The application layer also includes adapters that are used to translate information between the end devices and the message bus of the middleware layer. These adapters marshal, mediate, and map data between end devices and the field message bus.

The Interface layer includes data models, profiles, configurations and interaction patterns. The data model provides a common semantic understanding of the data that can be shared by all components of the architecture, depending on the data exchange requirements. Several data models are suitable candidates for use in the OpenFMB architecture. This project selected the semantic model of the International Electrotechnical Commissions standards 61970-301 [6], 61968-13[7], and 62325-301[8], that are known as the IEC CIM. This model which supports communications for Power System Management and Associated Information Exchange is quite large. Specific business processes and use cases require specific exchanges of data (interaction patterns) that use only subsets or profiles of the complete model that are called "based on the CIM". These profiles constitute a global data space that may be accessed by all components that participate in the OpenFMB architecture. A set of such profiles, were created by Duke Energy and by the SGIP for demonstration to the industry at various industry events in 2016. These profiles are available to the industry under the terms of an open source license. 
The publish/subscribe paradigm is a key feature of the OpenFMB Architecture. It allows CN to communicate with other CNs peer to peer, as well as to communicate with end devices and a master station. The OpenFMB supports the following the following publish middleware standards:

- $\quad$ Message Queue Telemetry Transport (MQTT) protocol (IEC/ISO RPF 20922)

- Data Distribution Services (DDS) protocol (OMG Data Distribution Services)

- Advanced Message Queuing Protocol (AMQP) ISO/IEC 19464

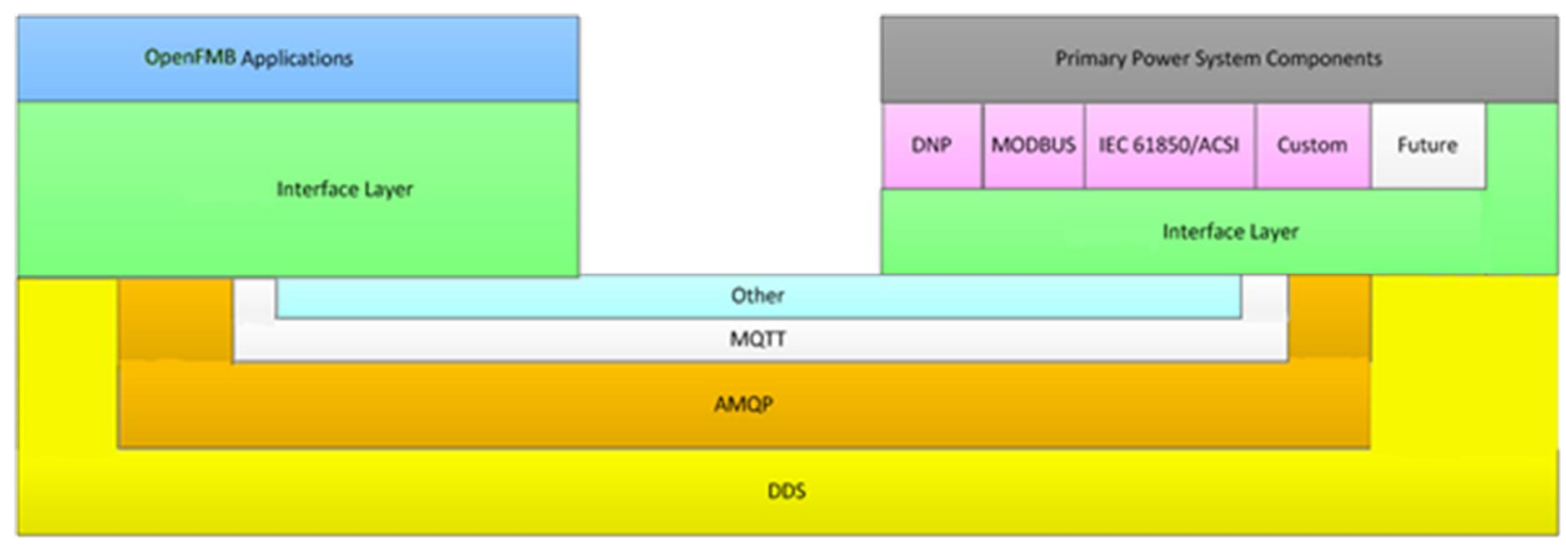

Figure 15: OpenFMB communications

Figure 15 illustrates peer to peer communications between OpenFMB Communications nodes. The Open Node on the right acquires data locally from power system comments using protocols such as DNP3, Modbus, IEC 61850 ACSI or custom protocol. Adapters in the right CN publish data to the global data space using one of the publish/subscribe protocols MQTT, AMQP or DDS. The Communication Node on the left may host an application that subscribes to the profiles in the global data space that it may need to access as input data required by the application functionality.

The example of Figure 15, may be easily scaled so that the application may subscribe to data from multiple power system components that is published by multiple communication nodes. The application may also publish results that are used by other applications running in additional communications nodes, realizing a truly distributed communications and control architecture without the bottlenecks and performance degradation challenges seen in traditional master/slave architectures.

\subsection{OpenFMB Methodology and Life Cycle}


The recommended methodology [9] for applying the OpenFMB in a smart grid setting is shown in Figure 16. The methodology consists of a set of steps represented by arcs that are followed sequentially. This methodology is used in conjunction with other processes for development of the smart grid control system proper.

\subsubsection{Business Case}

The first step in the process is the business case which identifies the business problem to be solved and the costs and benefits associated with the solution. This analysis leads to a go/no-go decision to pursue a solution to the problem.

\subsubsection{Use Case}

Following the decision to proceed with a solution, the next step in the methodology is the development of a use case that identifies the actors and systems that participate in the workflow associated with the business problem identified above. The flows of information between actors and systems are identified, along with the timing and frequency of exchanges. The exact information that is exchanged is specified. The OpenFMB methodology calls for documenting a use case as described in the IEC standard 62559-2. [10]

\subsubsection{UML}

The next step is the mapping of the exchanged message payload to the semantic model of the IEC CIM and the creation of the message payload structures. The mapping process relates each item of message payload to a corresponding class and attribute of the IEC CIM. In the event that the IEC CIM does not have class/attributes that correspond to the information in the message payload, the IEC CIM will be extended to include the required information and is called the extended CIM UML model. This is done with using Unified Modeling Language (UML) a modeling tool such as Enterprise Architect by Sparx Systems Ltd.

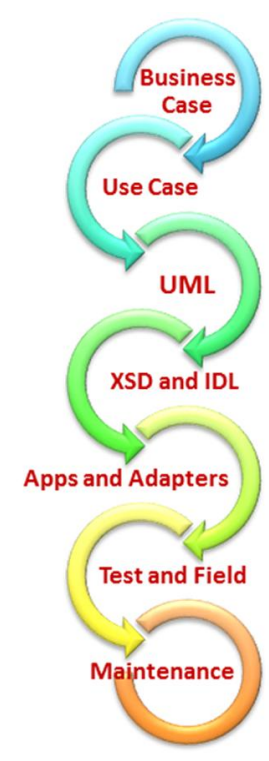




\subsubsection{XSDs and IDLs}

The message payload structures are produced as profiles or subsets of the extended CIM using the information maps and a serialization tool such as the open source CIMtool [12], or using a special plug in to the Enterprise Architect for exporting message XSDs. The resulting structures may be in XSD or IDL formats that are based on the IEC CIM UML model.

\subsubsection{Applications and Adapters}

This step consist of developing or configuring adapters to publish or subscribe to messages that are on the field message bus. The data in these messages are used as inputs to or outputs from the distributed applications that are hosted in the OpenFMB communications nodes described in section 4.2.

The SGIP PAP working group has created a repository [11] which hosts OpenFMB software, test cases, documentation and test simulators that are available under the terms of the open source Apache License Version 2.

\subsubsection{Test and Field}

These steps of the OpenFMB methodology involve the various forms of testing (unit, module, application, system, and end-to-end) that are typically used in the development of power system management software. Testing is followed by field work typically consisting of installation, commissioning, and cutover to operations.

\subsubsection{Maintenance}

This step involves keeping the software up to date including installation on a periodic basis of cyber security patches, bug fixes, revision upgrades, and finally decommissioning and replacement.

\subsection{Prior work with OpenFMB}

The SGIP created a priority action plan (PAP) to develop specifications for the OpenFMB. By November 2015, work and prototyping of the OpenFMB had progressed to the point where a demonstration of the concepts was possible at the SGIP Annual Meeting in New Orleans [13]. This demonstration consisted of a simulation of a microgrid on distributed communications nodes.

This was followed by a larger demonstration at Distributech in February 2016[14]. This demonstration was also included a simulated microgrid deployed on various CNs on the exhibition floor and a data link to an actual microgrid deployed at the Duke Energy Mt Holly Training Center. 


\subsection{Application of OpenFMB to Enhancing the Ability of the Grid to Support Renewables}

\subsubsection{Approach}

This section describes application of the OpenFMB to the recommended approach to enhancing the ability of the grid to support renewables that was introduced in section 3.3. The recommended solution approach is to create a hierarchical control scheme made up of a set of microgrids that report to a higher level control center such as an EMS or DMS. Each microgrid includes an aggregation of loads, critical loads, and renewable resources that are locally managed using an OpenFMB Architecture. Each microgrid includes a Microgrid Management System (MGMS) which forecasts its local loads and local production of power from its renewable resources. The MGMS will optimize the schedules for energy storage systems local to the microgrid, including schedules for net import or export from the utility grid at the point of common coupling (PCC). The resulting schedules for import/export at the PCC are communicated to a higher level utility DMS or EMS, in the day ahead time frame. The MGMS also reoptimizes during the operating day and also communicates any forecast schedule changes. The higher level EMS uses these inputs from microgrids in creating and updating its current operating plan. The higher level controller uses intra-day updates to adjust the current operating plan with the benefit of advanced warning of newly developed schedule changes (anticipatory schedules). Anticipatory schedules allow the utility operator to adjust plans to balance the system in the near term using utility resources which may require multi-hour lead times to deploy. For example with advance warning of a decline in solar production, the utility operator may be able to commit a unit that takes several hours to start. Without advance warning, this course of action may not be available to the operator.

\subsubsection{Business Case Analysis}

Business case analysis [15] examined the costs and the benefits of creating a microgrid to supply reliable power to critical loads. The cost components used in the study were taken from public sources and include typical values for different technologies. Several scenarios were considered for different geographies (urban vs rural), for different utility tariffs $(\$ 0.08, \$ 0.15, \$ 0.20 / \mathrm{kWh})$ and for different alternatives for supplying reliable power:

- 2nd feeder,

- large BESS plus solar PV,

- moderate BESS plus solar PV plus Diesel generator

The business case results identified the scenarios for which microgrids are economically justifiable. (As a side note, this points out that the case for microgrids with renewable power should be re-studied by a potential developer with the precise data for the site and using cost components obtained from current manufacturer quotations.)

\subsubsection{Refining the Use Case}

Three use cases were analyzed by the project: 
- Optimization of microgrid (grid connected and islanded)

- Unplanned transition from operating connected to grid to islanded

- Transition from islanded operations back to grid connected operations

The first use case was selected for demonstration at the NREL ESIF, at the Duke Energy site and the CPS Energy site. This use case is included is summarized in section 15.1.

\subsubsection{UML Model}

The information exchanges required by the use case were reviewed. Required changes were made to the UML model where needed for the demonstrations at NREL, Duke and CPS described in sections 7, 8, and 9.

\subsubsection{XSD and IDL}

The XSDs and IDLs that were created for the SGIP and Distributech were reused with only minor modifications. The project team participated in the work done for the SGIP and Distributech demonstrations as part of another project and was able to influence and include the required changes in the software that is available on the SGIP repository under the terms of the open source license.

\subsubsection{Applications and Adapters}

This work included integrating the Siemens MGMS with the OpenFMB and deploying and integrating adapters with various communication nodes and power systems components used in the respective microgrids. Please see sections 7, 8, and 9 for further details.

\subsubsection{Testing and Field}

The integration of the MGMS was tested in the OMNETRIC lab in Minnetonka using an ASE Test Set along with various software stubs. Commissioning and demonstrations at NREL, Duke and CPS are described in Section 7, 8, 9.

\subsubsection{Maintenance}

This step involves keeping the software up to date including installation on a periodic basis of cyber security patches, bug fixes, revision upgrades, and finally decommissioning and replacement. Please see Sections 7, 8, 9 as the arrangements for maintenance were different at each demonstration site.

\subsection{Cyber Security}

During the course of the project, the project team complied with all cyber security rules in place at the respective work sites: OMNETRIC, Duke, NREL, and CPS [18]. The scope of the project did not include advancing the state of the art for the security of the OpenFMB. However the project did follow existing cyber security rules and did include recommendations for follow on work to enhance the security of the OpenFMB [17], [18]. 
These recommendations include:

- Closing all unused device ports on all devices in the configuration

- Disabling all unused device operating system services

- Creation of a microgrid control system subnetwork with firewalls to the outside world

- Creation and maintenance of a blueprint showing all cyber equipment in the subnetwork

- Authentication \& authorization of all joiners/leavers to the network

- Maintenance of a certificate issuance/revocation capability

- Use of PKI technology for encryption

- Use of RTI DDS security for all data \& information flow

- Compliance with relevant parts of NERC CIP v5, standard [16] 


\section{Forecasting}

\subsection{Current State of Art - Load and PV Production Forecasting}

\subsubsection{Load forecasting and day-ahead PV production forecasting}

In the last decade, there is an increasing recognition that data-driven models can be used effectively to develop building baseline energy models [1-8]. These baseline models can be used for acquiring physical insight into building operation patterns, determining retrofit savings and for energy system fault diagnostics. Baseline energy modeling approaches are usually classified into two basic model categories: forward (classical) modeling, and inverse (data-driven) modeling. Typically, a model form is a priori assumed and measured data are used to find the parameters that provide the best fit for the chosen model form and data set. The forward modeling approach generally takes the physical parameters that describe the building as input, which can include building location, local weather, geometry, envelope construction materials, operational schedule, and HVAC system type, etc. The forward modeling approach is typically used in the design phase to facilitate building designers to make early design decisions. Inverse models use the monitored building energy consumption data (and possibly other available monitored behavior data) as inputs that are expressed in terms of one or more driving variables and a set of empirical parameters. Inverse models have been widely used in building retrofit projects [7], performance monitoring and system fault diagnostics [9], control strategy development [10], and online control applications [11]. In practice, inverse models trained by actual building energy consumption data can provide reliable load estimation and have been widely adopted for measurement, verification and ongoing commissioning of building performance [12].

Currently, the most popular approaches for inverse modeling of building energy performance are based on regression techniques. This is typically done in an ad-hoc manner relying on the assumption that the nonlinear energy behavior arising from complex multivariable relationships between ambient conditions, occupancy levels, and building operating conditions can be captured adequately through regression. Previous work [13] has introduced different linear regression methods to forecast building energy load and conducted studies using artificial neural networks to predict building cooling load [14]; utilized neural networks to estimate energy and demand savings from building retrofits [15,16]; developed temperature-based generalized Fourier series models to estimate hourly heating and cooling energy use in buildings [17]; use support vector regression methods to predict building energy load [4,18]; implemented Gaussian process regression baseline models to access total building energy load in the post-retrofit phase, which is a less time-consuming and efficient process [19]; and presents a Gaussian mixture regression model to predict building energy load with parameterized and locally adaptive uncertainty quantification for simulation data only [20]. A comparison of five whole building baseline models [10] that are widely used in the machine-learning field (i.e., Pulse Adaptive Model, multi-parameter changepoint model, mean week model, day-time-temperature model, and Lawrence Berkeley National Laboratory (LBNL) model) concluded that the Pulse Adaptive Model, LBNL model, and day-timetemperature model shows better performance compared to the change-point models. These models are 
recognized for representing the complex non-linear system with a limited number of physical parameters, but in general there is limited research that has investigated the integrated effectiveness, applicability and relative accuracy using data from real measurements.

\subsubsection{PV intra-hour forecasting}

Satellite and numerical weather prediction (NWP) are the preferred methods for day-ahead and intra-day PV forecasting. Statistical and learning methods based on historical data are also used for PV forecasting [21] although in the case of intra-hour or site specific PV forecasting, the use of publicly available satellite data is not very common due to the infrequent sampling interval (i.e. 30-60 minutes) and the low image resolution. For example, the NetCDF satellite data provided by GOES East and GOES West offer images of 430-605MB in time intervals of 45-minutes and 15-minutes with a pixel size of $6.8 \mathrm{~km}$ (Figure 17). The frequency of the data provided is insufficient to support 5- to 15-minute solar forecasting. A review of the state-of-the-art in solar forecasting is presented in [21].

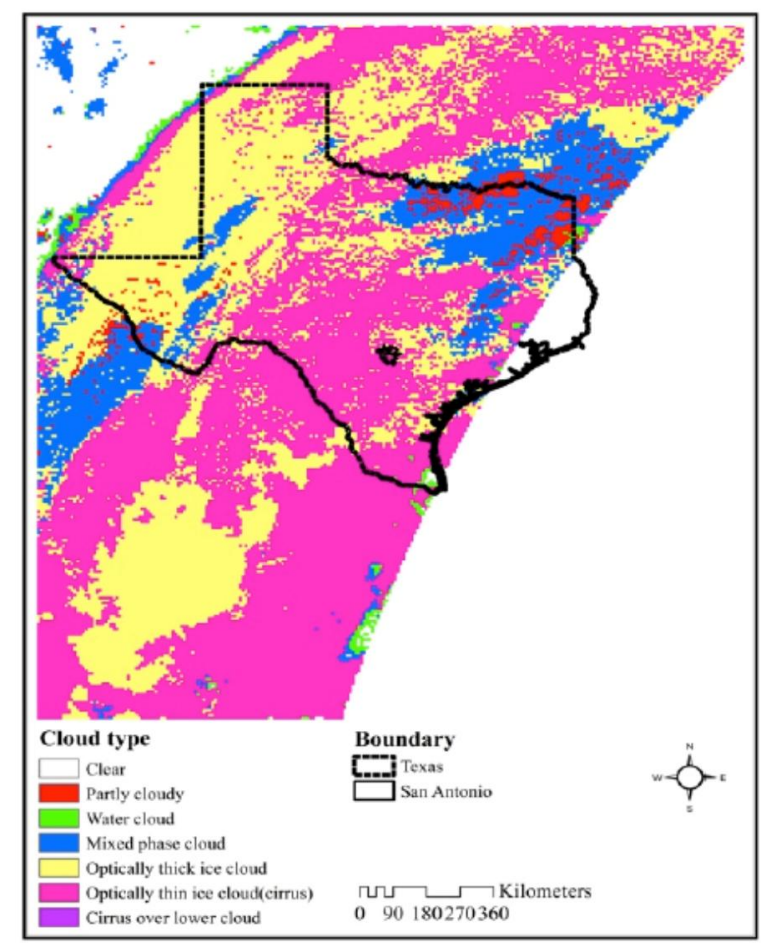

Figure 17 Typical GOES West Image of Texas

The correlation between satellite data versus ground observations from solar panels in a defined location can be quite inaccurate (Figure 18) based on actual measurements. This problem is further amplified in the NWP method due to larger sampling intervals and lower cloud development staging precision when compared to site-specific sky imaging cameras that produce high-resolution images at faster intervals (i.e., 10-15 seconds). However, it is noted that several basic assumptions regarding cloud shape and linear cloud movement vectors reduce the potential accuracy of forecasting based on sky imaging cameras [2223]. 
Many popular sky imager designs (e.g., Total Sky Imager TSI-440, cost \$ 7000) place the camera above and facing down on a reflective dome to capture their images (Figure 19). Due to the nature of this design, a "camera arm" and "camera housing" become constant artifacts in every image (Figure 19 B). Another artifact in each image is the "shadow band" tape used to avoid direct reflections from the sun into the camera (Figure 19 C). The "shadow band" does not remain in a fixed location in all images but moves in response to the sun's track over the day. Image processing software for commercially available sky imagers is not optimized for intra-hour forecasting but is typically designed to optimize the collection of meteorological information (e.g., \% cloud coverage). To mitigate some of these performance issues, an algorithm is used to mask the obstructions from the images, reducing the overall accuracy of the image provided (Figure 20)

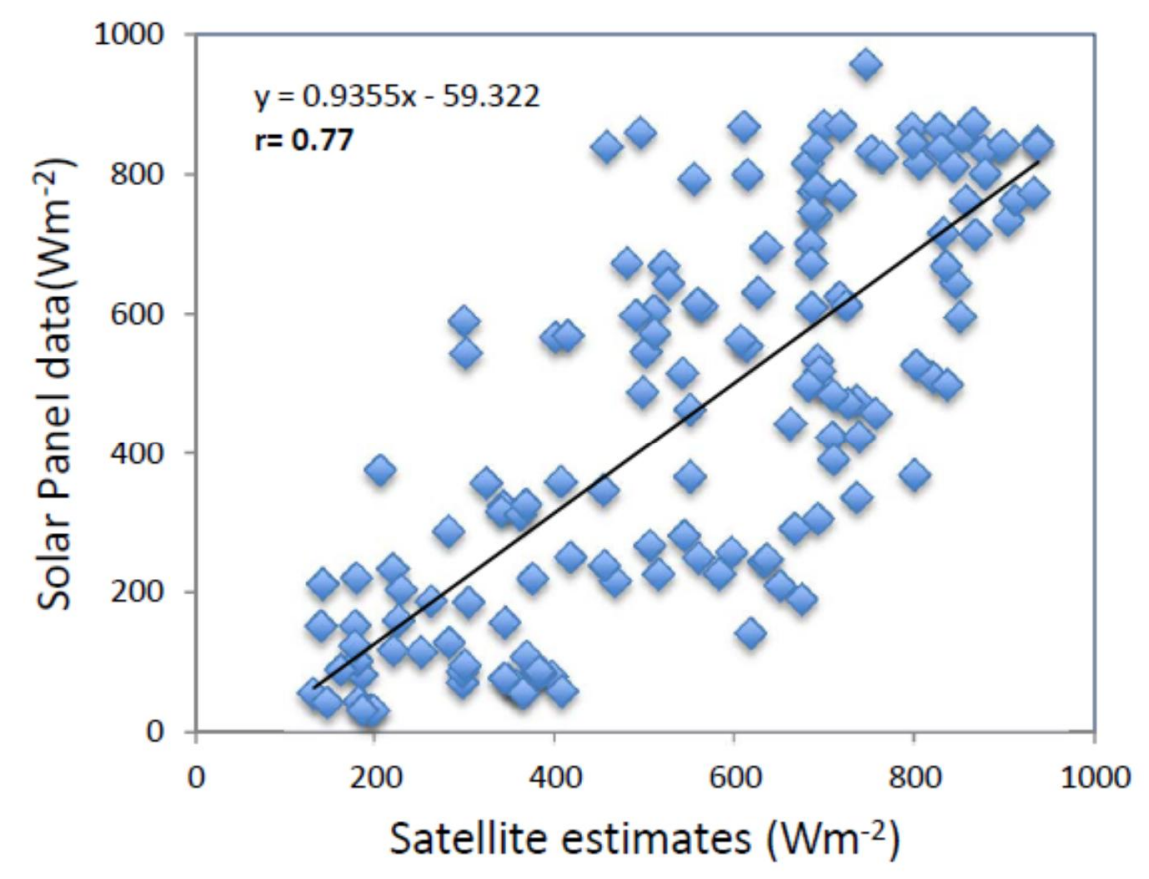

Figure 18 Comparison between satellite irradiance estimates and those obtained from a solar panel placed on the ground 


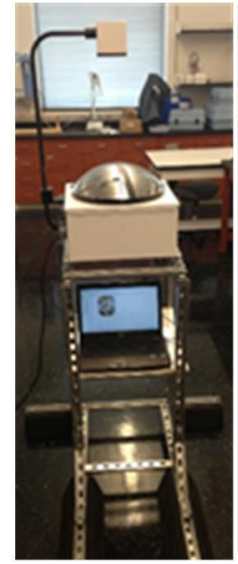

(a)

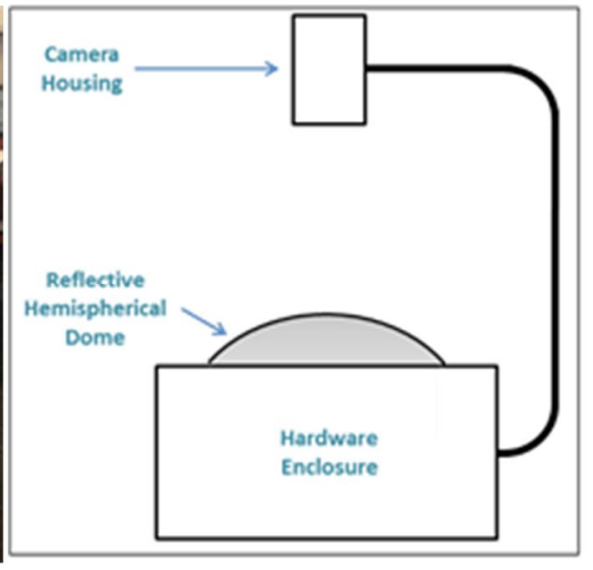

(b)

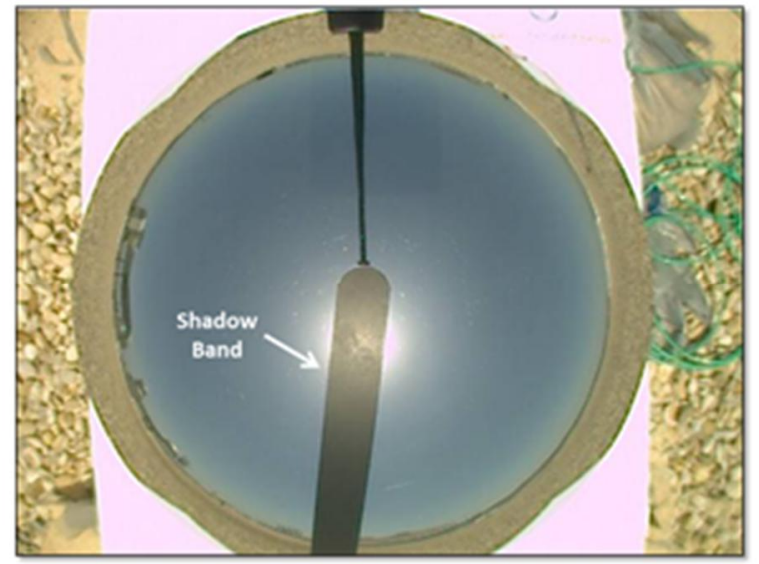

(c)

Figure 19: (a) Image of a typical sky imager, (b) scheme of a typical sky imager; and (c) artifacts placed in the image due to the camera and the shadow band used to avoid direct reflection of the sun on the camera

After correcting for image artifacts, the next data processing step is to represent the sky at a given height by calculating the maximum cloud base radius. The radius is usually obtained from a nearby ceilometer (Figure 21) located close to the sky imager deployment (e.g. an airport) and the minimum sky altitude that is a user-defined value. When the sky altitude is set to zero degrees, the radius of the sky image is infinite. The size represented by each pixel is then calculated. For detecting clouds in an image, different intensity values for clear and cloudy areas are assigned with the difference in intensity used to isolate the clouds.

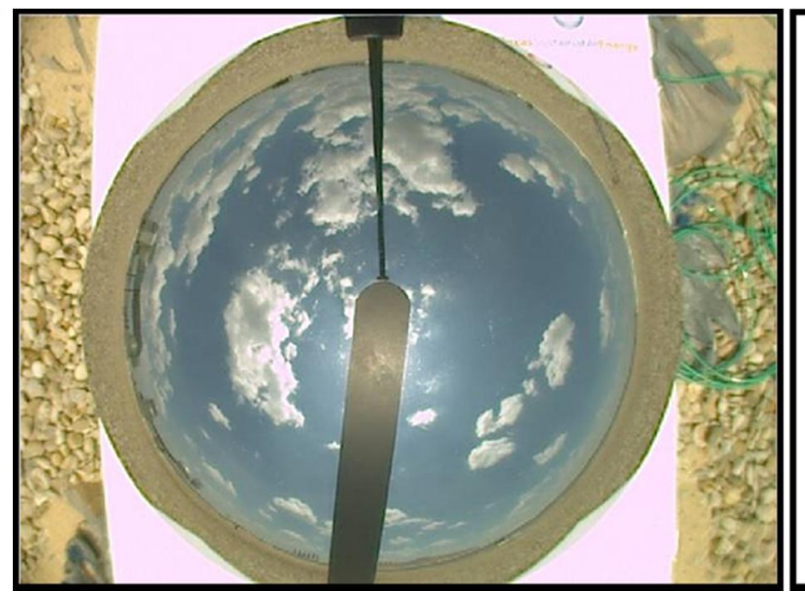

(a)

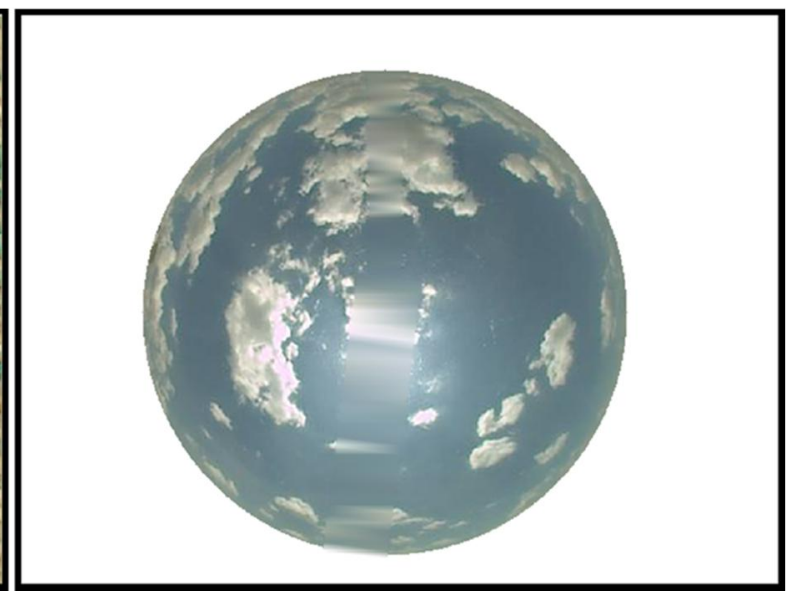

(b)

Figure 20: Typical sky image of a partly cloudy day (a) before; and (b) after masking the obstructions 


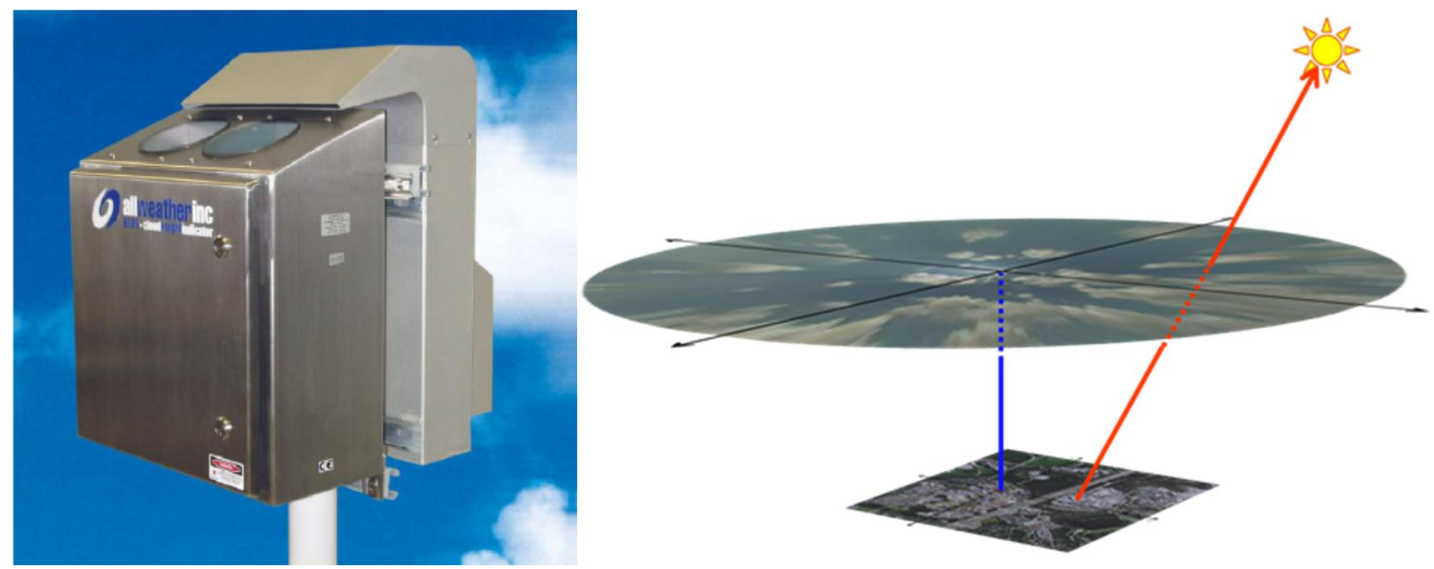

Figure 21: (a) Typical ceilometer and (b) scheme the calculation of cloud lower height

Three methods commonly used for predicting future solar irradiation include phase correlation between two images [24], cross correlation (Pearson's cross correlation coefficient) between rectangular and polar sub-frames within an image [25], and local feature-based regression models [26, 27]. The first two methods are homogeneous area-based approaches comparing intensity characteristics of block- or sectorwise areas while the third one uses local descriptors (such as viewpoint, scaling and affine transformation) of cloud objects and features. The regions, sub-frames or features identified in consecutive images are then projected into the future in the direction and with the characteristics specified by area-based or local feature-based methods. Opportunities that may exist to modify one or more of these approaches to increase both the accuracy of the forecasting methodology and reduce costs are described below.

\subsection{UTSA Advanced Load and Solar Forecasting Methodology}

\subsubsection{Overview}

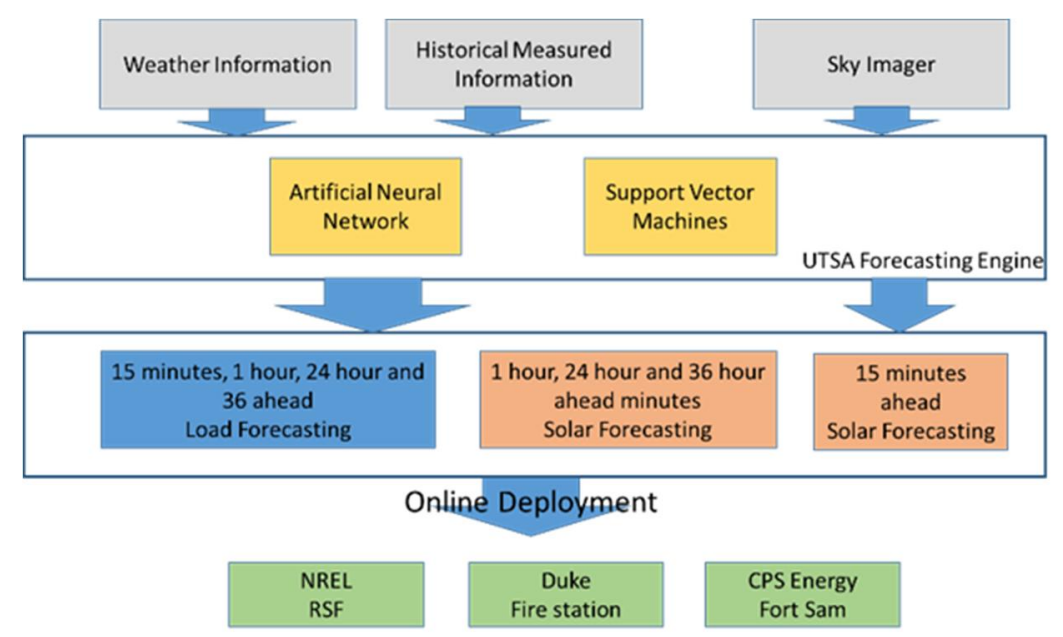

Figure 22: General flow of information and analyses for the UTSA solar and building load forecast capability 
The UTSA load and PV forecasting approach integrates and innovates on several aspects compared to the more traditional approaches discussed in 5.1.1. Two artificial machine-learning methods, Artificial Neural Network and Support Vector Machine, are specially designed for the on-line forecasting. They were selected among many other popular black box approaches, including Gaussian Mixture Model, Gaussian Process Approach, and Autoregressive-Moving-Average Model. The inputs for providing the 1- hour ahead or longer forecast include weather information and historical measured information (historian data) for the building load and PV systems. Intra-hour forecasts (less than 1 hour) for buildings use the same information as the longer forecasts but may also include Sky Imager input to enhance the forecast. Sky Imager input was used in test demonstration mode to assess technology integration and deployment, communication and data processing procedures at NREL and offsite for Fort Sam Houston. At NREL, Direct Solar Irradiance (DSI) values were obtained for five separate days. Forecast values were used to provide information of an impending irradiance drop and accompanying ramp in PV array power output. The forecasting methodology uses observations from the Automated Surface Observing System (ASOS) and cloud cover forecasts from a third-party source (Weather Underground) as inputs to the ANN/SVM codes that predict the 36 hours ahead time series in 15-minute increments. Models are specifically divided into two stages: online deployment and offline optimization. The online predictions are forecasted using prefixed parameters which guarantee the fast simulation speeds (i.e., within 1 minute) needed to provide a sequence of 5-minute forecast intervals followed by a sequence of 15-minute forecast intervals and publishing from the 15-minute ahead to 36-hour ahead window. The inputs are limited to temperature and historical information only, owing to the weather forecast source and on-line computation time constraints. The prefixed parameters are constantly updated once the operator initiates the offline optimization. This optimization utilizes the cross validation to verify the model stability according to the grid search of a range of parameters. The most accurate forecasts substitute the outdated "prefixed" parameters with the new "prefixed" parameters as they become available. Forecast results are demonstrated using NREL RSF1 and RSF2 buildings, Duke McAlpine Fire Station, and the CPS Energy Fort Sam Houston Library location.

\subsubsection{Description of Methodologies}

\subsubsection{Load Forecasting}

The UTSA advances to forecast building load and PV day-ahead forecasting have been driven by statistics methodology and machine learning methods (Fig. 5.7) that incorporate Artificial Neural Networks (ANN) and Support Vector Regression (SVR). ANN is based in a network model connecting input, hidden and output layers of neurons, and the appropriate learning algorithms. In this project, feed forward neural networks (FFNN) of a single layer and a double layer configuration were explored. However, due to over-fitting problems, accurate forecasts were not provided from the double hidden layer structure, similar to the layers tuning tests in building load forecasting. The FFNN was modeled as one input layer, one hidden layer with more than 20 training neurons and one output neuron (i.e., the prediction). The model was implemented using the NeuroLab Library 0.3.5 in Python, the transfer functions were the hyperbolic tangent sigmoid functions for each layer. FNNN trained with gradient 
descent back-propagation algorithm. FFNN's weights were learned by minimizing Sum Squared Error (SSE). To select the specific number of hidden neurons, the training algorithms were performed on multiple epochs of historical data. For each epoch selected (e.g., one week), 75\% of the data was used for training and the remaining $25 \%$ was held out for validation. The prediction was optimized using all input data normalized between 0 and 1 . At least 50 epochs are simulated until the calculated SSE reaches the setting error goal of 0.001 .

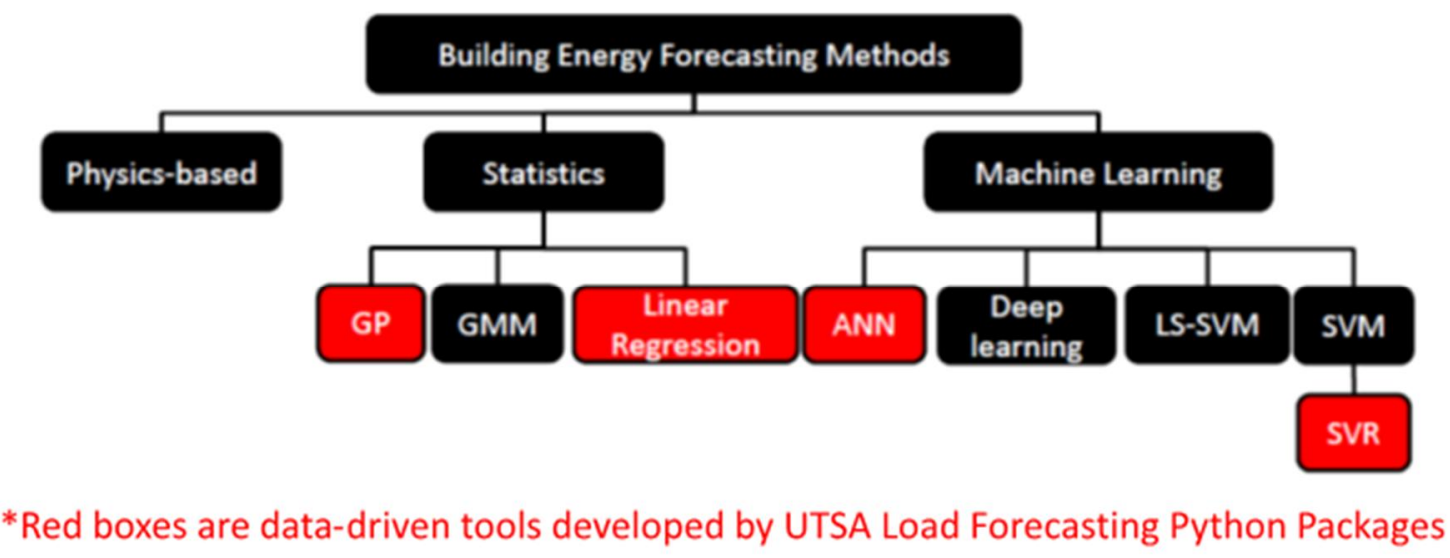

Figure 23: Scheme of the building energy forecasting methodology developed by UTSA.

Support vector machines are statistics learning tools widely used in classification and regression problem. For support vector regression (SVR), a data set is firstly transformed into a high dimension space, and predictions are discriminated from training data as a "tube" enclosed with a desired pattern curve with certain tolerances. The support vectors are the points that mark the margins of the tube. The commonly used choices of kernel function used are linear, polynomial, and radial basis function (RBF). Scikit-learn Library 0.15.0 in Python was used in this project. The RBF uses a 3-degree kernel function. The parameters were determined with 10 -fold cross-validation based on mean square error.

Two building samples were used to illustrate how to forecast building load and PV production in 15 minute intervals for 15-minute ahead, 1-hour ahead, 24-hour ahead head and 36-hour ahead: the NREL Research Support Facility (RSF) Buildings 1 and 2, and the McAlpine Fire Station.

The initial forecasting activity examined a 137,000 ft2 RSF2 building in NREL (Golden, CO) that houses more than 500 occupants. The RSF2 building energy use budget is $10 \mathrm{kWh} / \mathrm{m} 2 / \mathrm{yr}$. ( $34.4 \mathrm{kBtu} / \mathrm{ft} 2 / \mathrm{yr})$ based on the results of the design modeling. The expected RSF2 usage is 1.0 million $\mathrm{kWh} / \mathrm{yr}$ that is offset by the on-site solar energy systems. Building orientation, insulation, passive solar design and external overhang contribute to a stable load profile during weekdays, but substantial variations occur on weekends (Figure 24). 

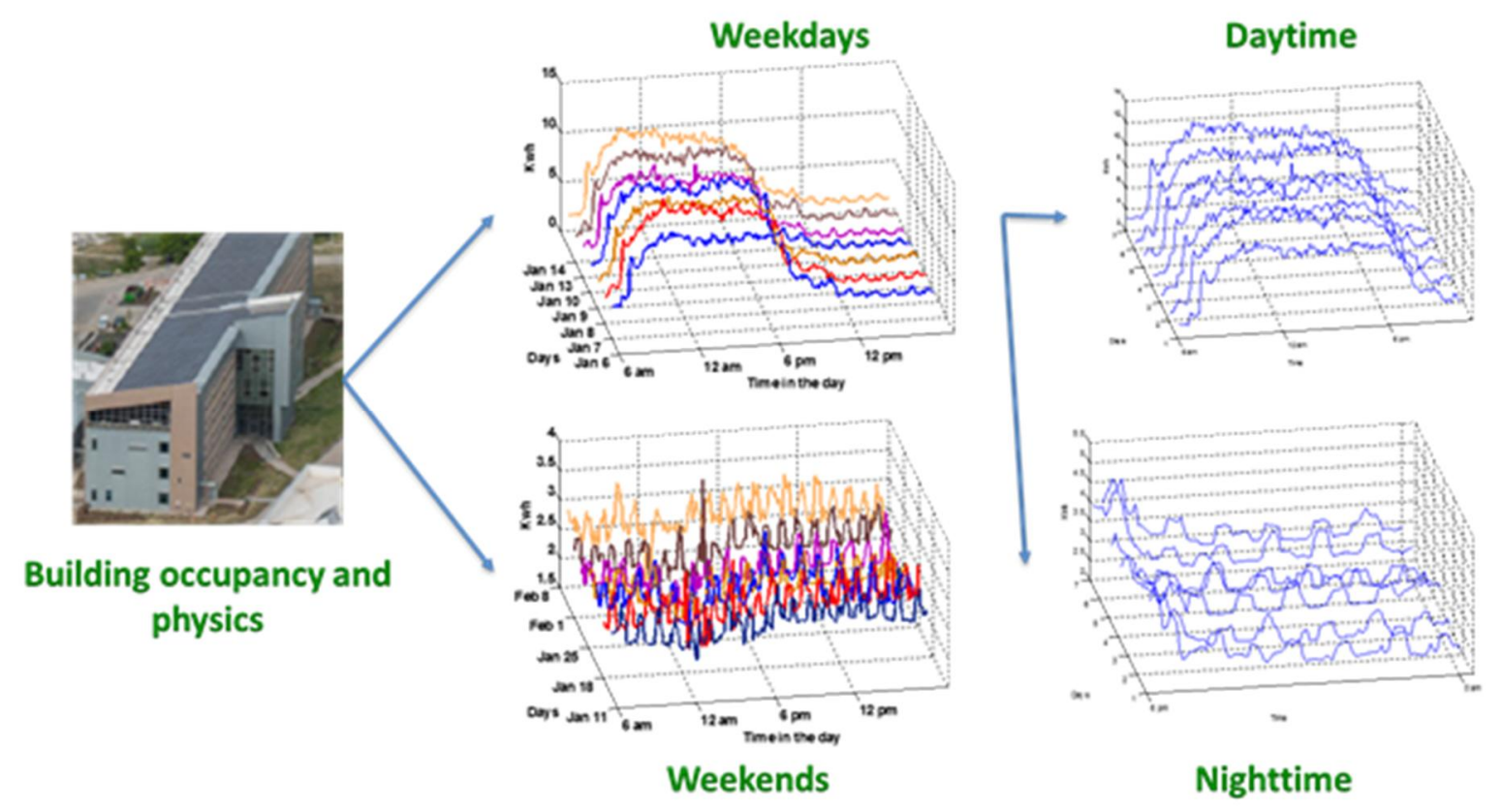

Figure 24: RSF Building Load at different time periods

The input set for the one step ahead forecast for building load (e.g., 15-min, and 1-hour ahead) included time information (outdoor air temperature, relative humidity, and the wind speed) and one to four inputs of the historical building electricity consumption. The main steps in obtaining the forecast included interpolation and filtering, data mapping, a selected training period and the design of the trained model (Figure 25). The 24-hour ahead case needed the information of the load measured at the exact same time on the previous day and over the previous 5 most recent historical days.

We recommend using a maximum of 15 working days (three work weeks) in the inputs to avoid an overfitting model that adversely impacts forecast performance. For the NREL RSF2 load forecasting, a demonstration period lasting 4 months was used (from 9/1/2015 to 12/31/2015). The process corrects the 
- Historical Load

- Outdoor air temperature

- Wind speed

- Relative humidity

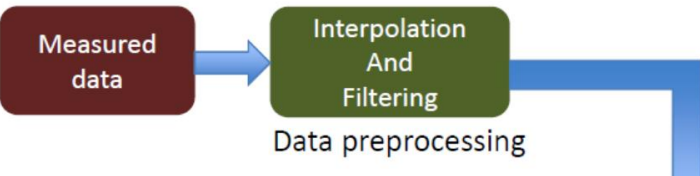

Select

Training

Period

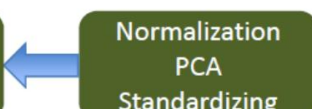

Standardizing

Data mapping

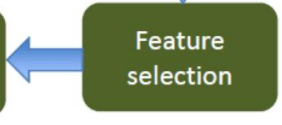

selection

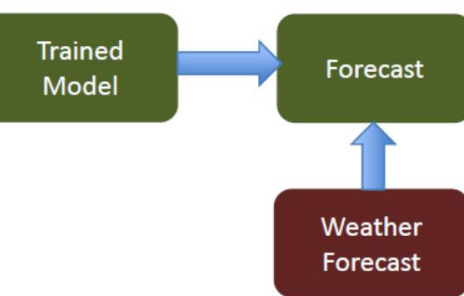

Figure 25: Scheme of Load Forecasting Process

wrong labels, revises the load calculations, and eliminates the periods without weather information. The main matrix to evaluate the forecasting performances were calculated by Mean Absolute Percentage Error (MAPE):

$$
\operatorname{MAPE}(\%)=\frac{1}{N} \sum_{i=1}^{N} \frac{\left|y_{i}-y_{p}\right|}{y_{i}} \times 100
$$

\subsubsection{Day - Ahead Solar Forecasting}

For day-ahead solar forecasting the goal was to implement data-driven methods for building load forecasts 24-hours and 36-hours ahead using a similar methodology as that used for building load forecasting based on the ANN and SVR methods (Figure 26).

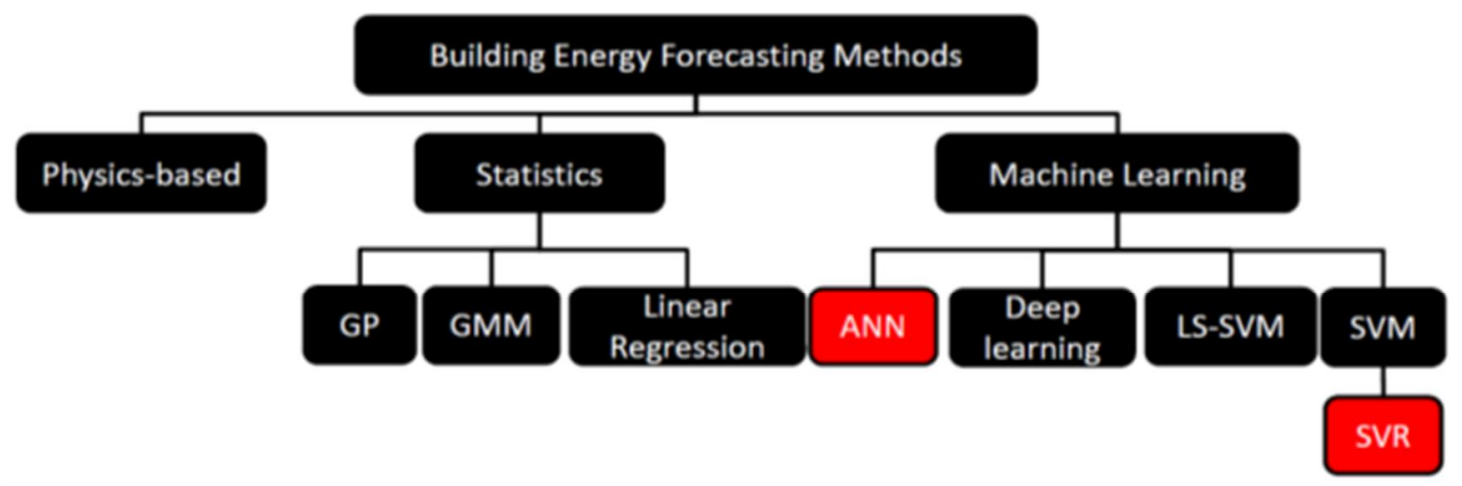

*Red boxes are data-driven tools developed by UTSA Solar Forecasting Python Packages

Figure 26: Scheme of the day ahead solar forecasting methodology developed by UTSA 
In this case, the data driven approach is based on historical PV, air temperature, solar radiation, relative humidity and solar position inputs (Figure 27).

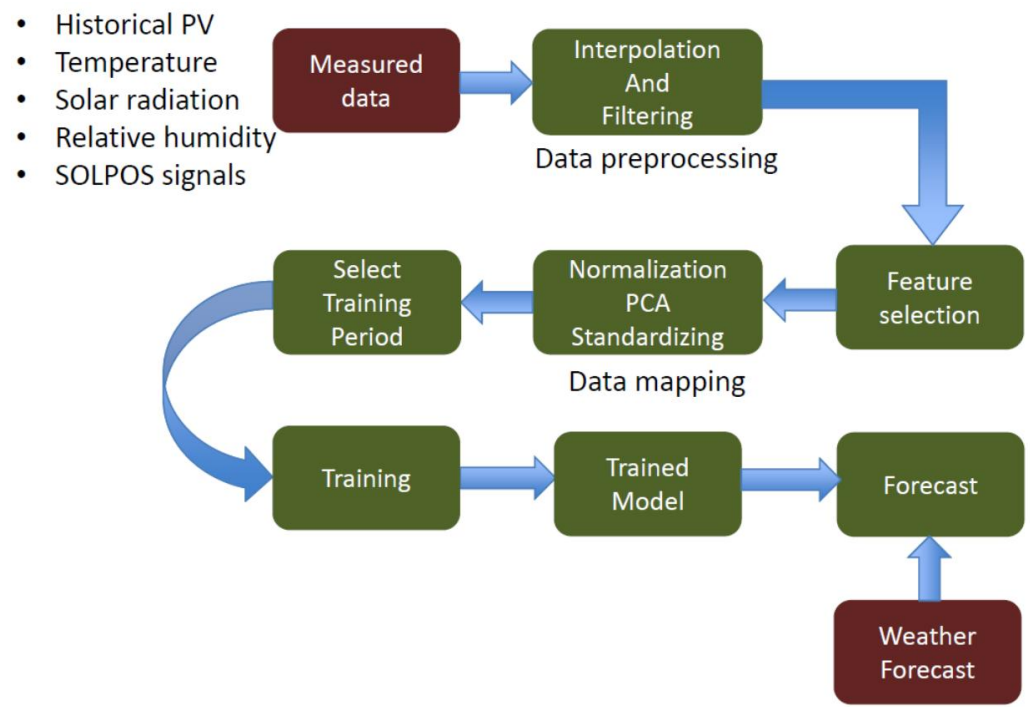

Figure 27: Scheme of the load forecasting process

The UTSA PV forecasting methodology has incorporated many of the advances developed for load forecasting including the methods being designed for the on-line forecasting, an offline optimization to update the model parameters by utilizing cross validation, and a hybrid approach combining the machine learning forecasts and the persistent predictions. In addition, the PV forecasting models can accommodate the missing or negative values in the actual PV production and has an option to input cloud cover forecasts (currently not available from the online weather forecast) to differentiate between cloudy and sunny days in the training set and prepare the models separately for predictions under different weather conditions. Although the models are tuned to be as flexible as possible for multiple types of model inputs, it is strongly recommended to include as many as solar-related input signals as possible. As expected, predictions improved markedly for PV production when model inputs were strongly correlated with solar irradiance and clear sky conditions.

\subsubsection{PV intra-hour forecasting}

This project introduces a newly designed, low cost ( \$ 500), sky-imaging system designed to provide forecasted output to system operators for improved resource allocation. The system has an unobstructed view of the full sky and employs a novel methodology for intra-hour cloud forecasting that improves upon some assumptions of cloud shape and cloud movement (Figure 28). The novel approach to the detection of cloud motion distinguishes the motion and motion characteristics of individual clouds. The system improves the predictability of distributed solar generation through the use of an independent irradiance estimation method capable of making forecasts for spatially scattered locations. 


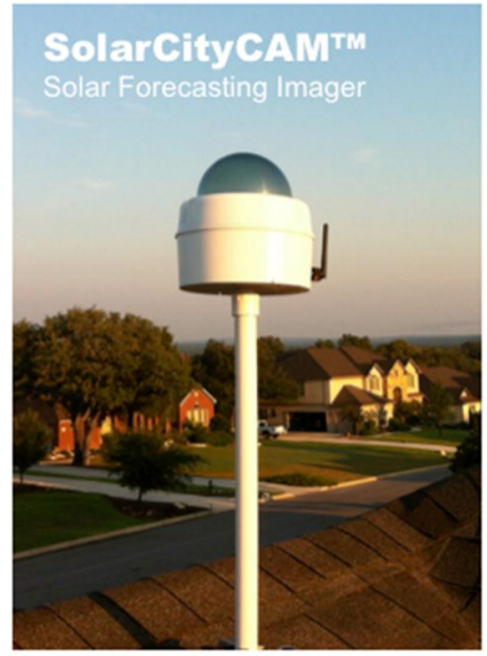

(a)

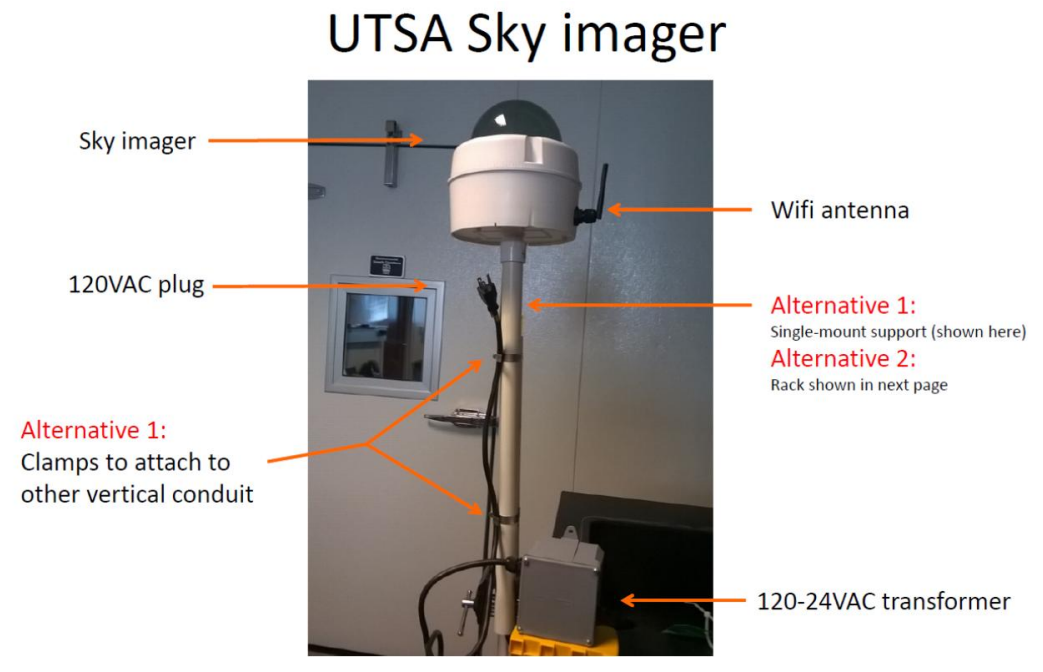

(b)

Figure 28: (a) View of the UTSA Sky Imager placed on the roof of a house and 9b0 scheme of the UTSA sky imager and main components and alternatives

As mentioned earlier, the method employed for the treatment of images is a special case of the local feature-based regression model which identifies and stores features of individual clouds in order to make future predictions of cloud locations. The first step in the process is to capture the sky images with the sky imager using a Fusion Technique and then perform post processing or the images to avoid sun disturbance of the cloud images and to remove unnecessary information (Figure 29). This post processing effort allows image processing to focus on relevant information in the image (i.e., cloud and sky pixels).

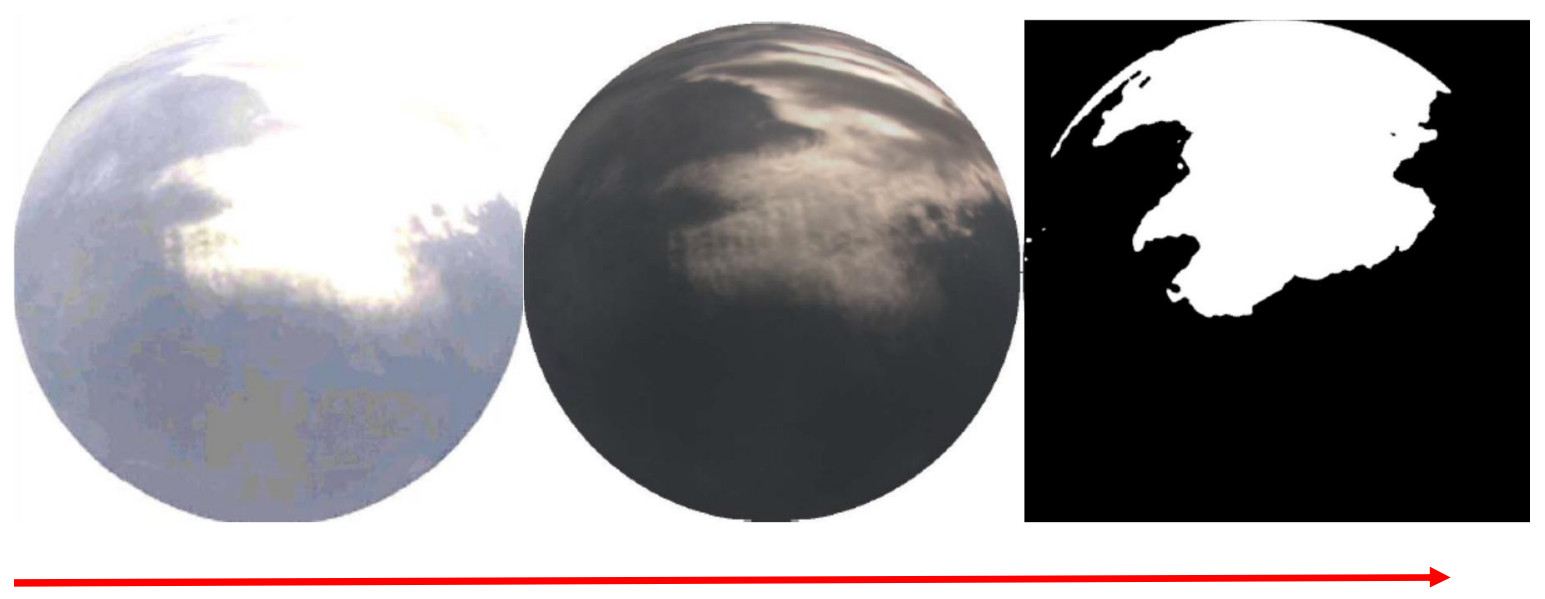

Figure 29 Scheme of the treatment of on sky image using the Fusion Technique developed by UTSA

Additional processing entails a geometric transformation to convert an image in polar coordinates to Cartesian coordinates (Figure 30). This transformation is needed to obtain the projection of the sun's rays onto the ground for irradiance calculations. The transformation process includes an assessment of camera matrix and distortion coefficients and the transfer of the images to a Cartesian plane image used for 
irradiance calculations. The motion of each individual cloud is used to define the search path in which to look for individual cloud matches. The boundaries, centroids and characteristics of whitespace regions in the binary image are defined within the Cartesian coordinates (Figure 31).

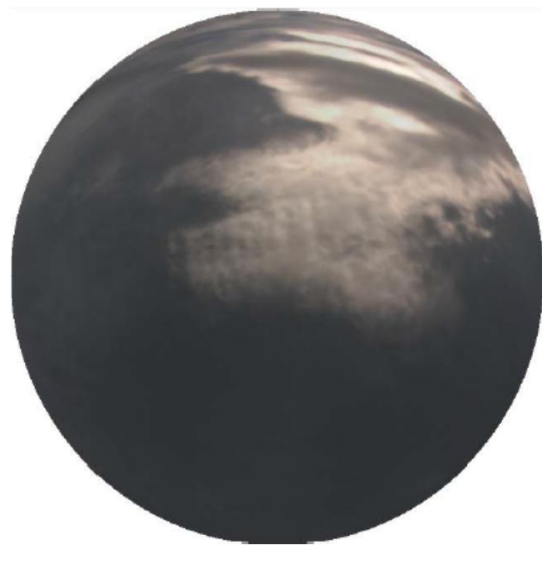

(a)

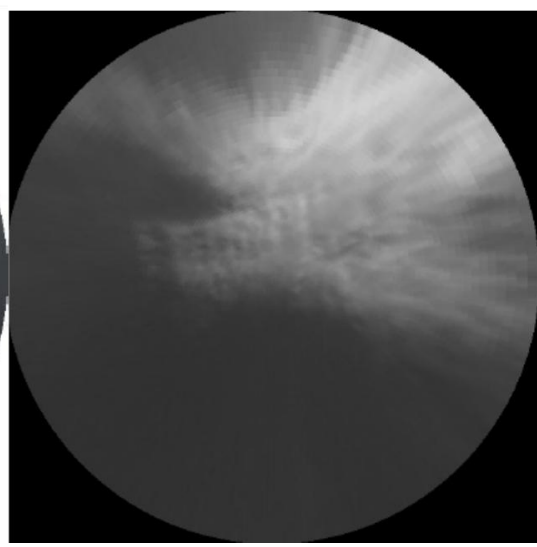

(b)

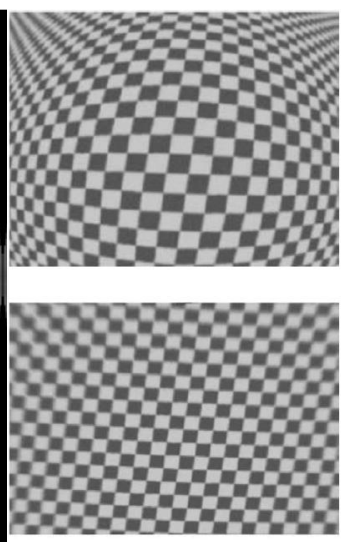

(c)

Figure 30: Sky Image (a) before; and (b) after the transformation into a Cartesian plan image. (c) representation of the transformation process (top to bottom)

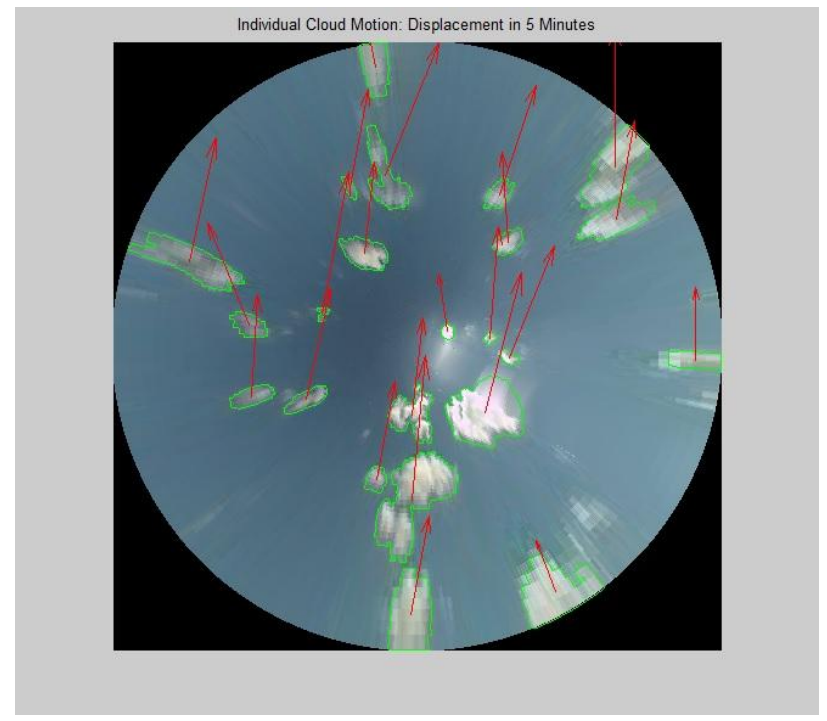

Figure 31 Vectorization of the individual cloud motion by analyzing consecutive sky images

After identifying each individual cloud within both the current and the previous image, each cloud in the previous image is overlain onto the current image and the motion (distance and direction) to every cloud in the current image is calculated. This process is repeated until each cloud in the previous image has been overlain and analyzed. In most cases, the median of all cloud matches most closely resembles the actual general motion of all clouds, and the desired matching cloud is the shortest distance away from the 
reference cloud. For this reason, only the closest centroid distance for both the movement speed and direction is preserved.

After the general direction for the current time clouds have been established, the following procedure is used to generate the motion characteristics of each individual cloud. The first part of the procedure is to define the cloud motion search path. When a previous cloud is overlain onto the current image a search path is defined at the cloud centroid. The purpose of the search path is to exclude clouds that lay beyond this region from the cloud matching process. Figure 32 shows the results of the cloud matching process, where the green highlighted region is the current cloud. The leftmost and the middle images show the detection of single cloud matches and the image on the right shows an example of a current cloud with two matching results.
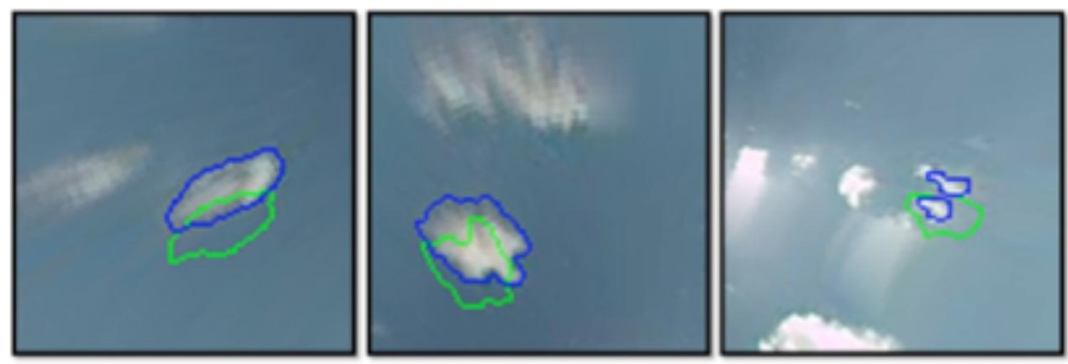

Figure 32: Cloud match results from current time for different clouds

The last step in the process is to create the future images from which predictions can be made. Each cloud (white) is individually cropped out of the image and placed in a new location based on the direction and distance travelled and re-sized according to the change in area. To produce an irradiance measurement or a cloud shadow projection, a ray tracing procedure is performed from the ground pixel location through the cloud image layer to the sun (Figure 33).

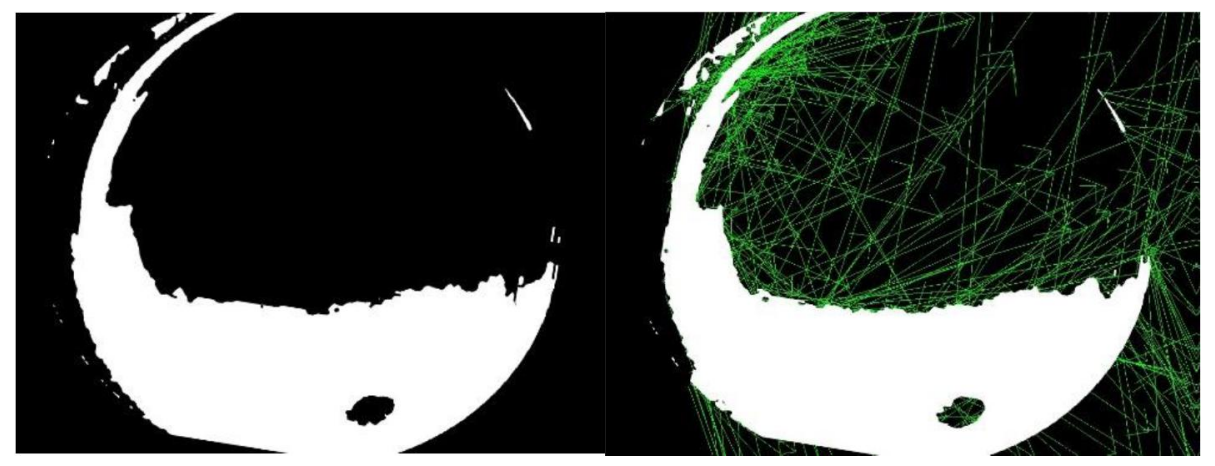

Figure 33: True North orientation based on physical location and 5-min vector forecast based on previous vectors from feature points

The tracing procedure provides a pixel intensity value from the sky image for any point on the ground (Figure 34), which is another novelty in this approach, thereby significantly enhancing the resolution. A binary NDI on/off signal from the binary image is used for ramp prediction, while a second method gives a DNI value range from the subtracted red to blue ratio image, which results in more accurate NDI 
estimates. Both methods are then multiplied by a clear sky NDI reference curve to provide the actual NDI estimate. Cross-hair approaches are used to determine the value for the NDI estimation when the pixel location in the sky image is situated in the interpolated shadow band region between the sky and the cloud.

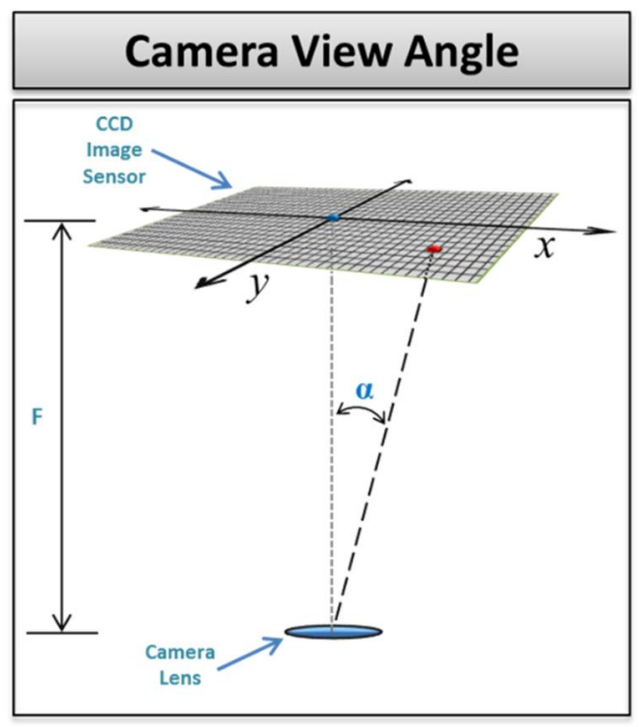

Figure 34: Scheme of the tracing procedure to provide a pixel intensity value from the sky image for any point on the ground

The diffuse horizontal irradiation (DHI) calculation begins by scanning the entire binary image and detecting all the cloud pixels. For each detected cloud pixel, the subtracted red to blue ratio image value is acquired and divided by its distance from the reference point squared to give more weight to pixels closer to the reference pixel (Figure 35). The values are then summed and normalized on a scale from zero to $20 \%$ of the DNI reference. Lastly, the estimated DNI and DHI are summed to produce a GHI value.

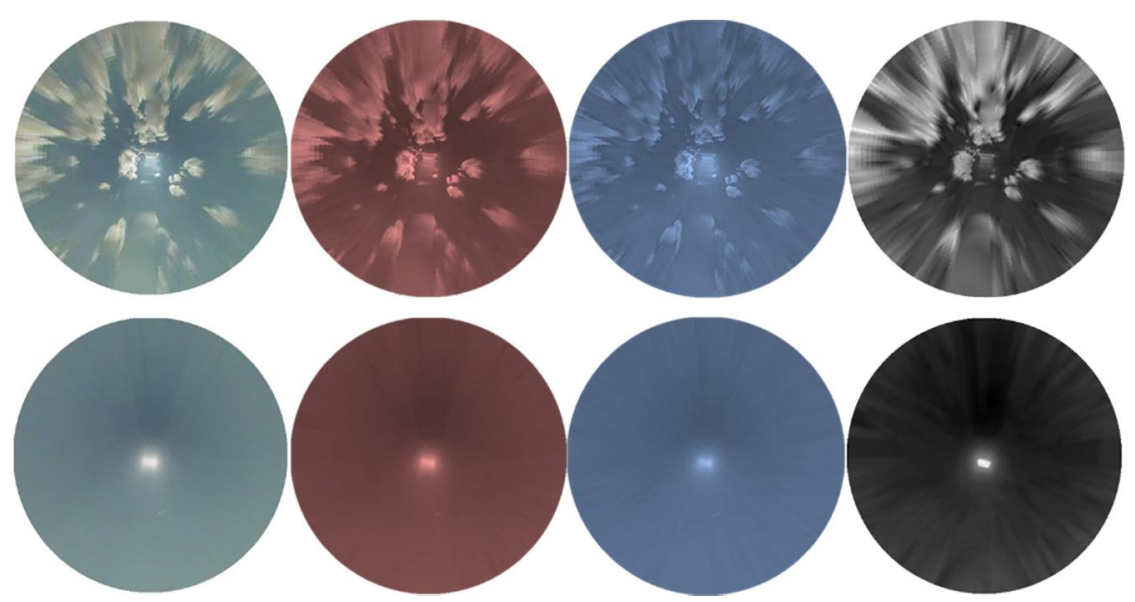

Figure 35: Red to blue ratio calibration normalized (0-255). In a clear sky blue is much larger than read, and in a cloudy sky blue is more equal to red 
A Monte Carlo simulation is performed (Figure 36) that provides grid operators with an analysis of the uncertainty associated with each forecast. After the first pass through the solar forecasting engine, five possible variables with uncertainty need to be analyzed: cloud base height, individual cloud speed, individual cloud direction, individual cloud delta area (change in area), and DNI/DHI reference.

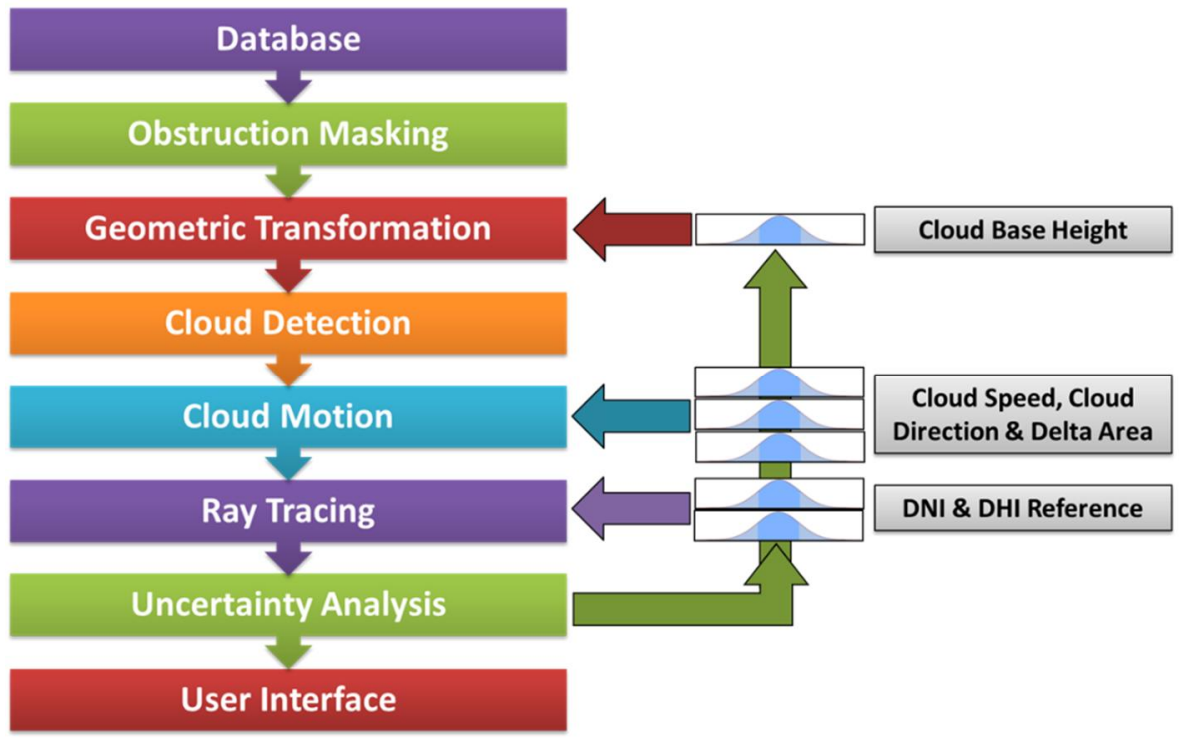

Figure 36: Scheme of the Monte Carlo Simulation

A user interface is also provided to show the cloud cover time lapse on the area where the PV systems are deployed, a graph showing the real GHI and forecasted up to 15-minutes embedded in different confidence bands, and a table where the values are exposed with different confidence bands (Figure 37).

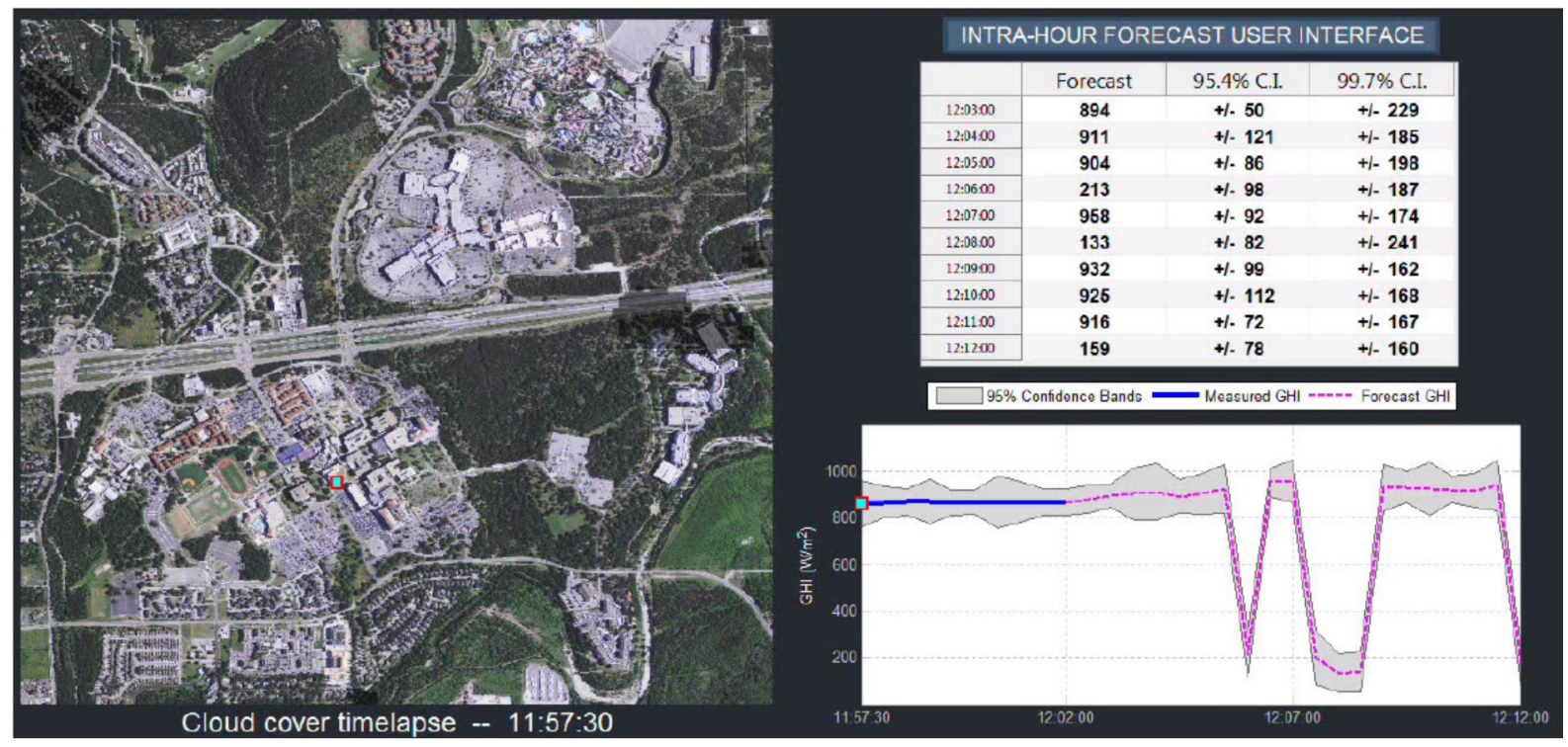

Figure 37: Capture of the user interface provided by UTSA for the short term forecasting system 


\subsection{Offline Model Validation of Forecasting}

\subsubsection{Load Forecast}

\subsubsection{NREL RSF Buildings}

Results for weekdays in NREL are summarized in Table 5.1. Predictions are made every 5-minutes with 15-minute ahead, 1-hour ahead, 24-hour ahead and 36-hour ahead simultaneously. A hybrid approach is further developed for the one step ahead forecast in the project. It is a weighted sum between the machine learning forecasts and the persistent forecasts. The accuracies of the forecasting results are summarized in Table 3.

Table 2: NREL Load Forecasting MAPE Statistics

\begin{tabular}{c|c|c|c}
\hline RSF 1 & ANN & SVR & $\begin{array}{c}\text { Hybrid (SVR + } \\
\text { Persistent)* }\end{array}$ \\
\hline & & & \\
\hline 15-min ahead & $7.31 \%$ & $6.23 \%$ & $5.04 \%$ \\
\hline 1-hour ahead & $7.92 \%$ & $7.51 \%$ & $6.63 \%$ \\
\hline 24-hour ahead & $9.11 \%$ & $8.16 \%$ & - \\
\hline 36-hour ahead & $10.24 \%$ & $9.93 \%$ & Hybrid (SVR + \\
\hline RSF 2 & ANN & Persistent) \\
\hline 15-min ahead & $9.71 \%$ & & $7.95 \%$ \\
\hline 1-hour ahead & $14.20 \%$ & $9.02 \%$ & $12.42 \%$ \\
\hline 24-hour ahead & $13.23 \%$ & $13.33 \%$ & - \\
\hline 36-hour ahead & $13.46 \%$ & $12.83 \%$ & - \\
\hline
\end{tabular}

The table illustrates which method has the best and most consistent performance for the RSF buildings in different forecasting windows. The proposed hybrid model and common applied machine-learning methods, ANN and SVR, perform similarly but the hybrid model consistently shows better results. A comparison of the longer forecast results also show similar performance among the methods. However, the forecast error in Table 4 comparing results with the hour-ahead forecast is statistically distinct (i.e., MAPE values are increasing gradually comparing hour ahead forecast for the same buildings). This implies that the temporal variance in the forecast window has significant impact on the predictive power of both machine learning and hybrid models.

Table 3: NREL RSF Building Long Term Forecasting MAPE Statistics

\begin{tabular}{c|c|c}
\hline RSF 1 & ANN & SVR \\
\hline 24-hour ahead & $9.11 \%$ & $8.16 \%$ \\
\hline
\end{tabular}




\begin{tabular}{c|c|c}
\hline 36-hour ahead & $10.24 \%$ & $9.93 \%$ \\
\hline RSF 2 & & \\
\hline 24-hour ahead & ANN & SVR \\
\hline 36-hour ahead & $14.23 \%$ & $14.83 \%$ \\
\hline
\end{tabular}

The MPAE results in both tables illustrate the different performances for different buildings within the same forecast window. The differences result from fundamental differences in energy usage for RSF1 and RSF2 . We postulate that the large forecast errors of RSF2 (>10\%) can be reduced with additional occupancy-related signals such as working schedules. The accuracy of the current models are limited by the available input energy characteristics even where the same type of inputs and parameter optimization techniques are used. Buildings are built with various materials and are often operated with different equipment schedules. From the results of RSF2, it is obvious that the models in the test cases are reaching their limitations unless more consumption-correlated input signals (e.g., occupancy behavior signals) are incorporated within the input data.

\subsubsection{Duke McAlpine Fire Station}

Duke Energy has modified an existing facility to create a technology innovation laboratory in the McAlpine Creek neighborhood in Charlotte. The test case building used in this study is the Charlotte Fire Department Station that is a retrofitted building in use since 1981 (compared to the newly designed NREL RSF buildings). Our initial assessment suggested that because of the building size and limited energy consumption the forecast predictions would be more straightforward compared to the NREL RSF buildings, however, the data is quite irregular because of load dynamics associated with the emergency response mission that even the one step ahead forecast cannot guarantee a reasonably accurate 15-minute and 1-hour ahead forecast. The input parameters are very similar to those specified in Section 5.1.1 and three months of results are presented in Table 5.

Table 4: Fire station load forecasting -- MAPE Statistics

\begin{tabular}{c|c|c|c}
\hline Fire Station & ANN & SVR & Persistent \\
\hline 15-min ahead & $17.53 \%$ & $17.15 \%$ & $18.30 \%$ \\
\hline 1-hour ahead & $19.00 \%$ & $18.19 \%$ & $21.72 \%$ \\
\hline 24-hour ahead & $19.40 \%$ & $18.78 \%$ & - \\
\hline
\end{tabular}

Variance differences summarized in Figure 38 use 1.5 interquartile range (99\% confidence interval) boxand-whisker plots. Large variances are observed in McAlpine fire station, with a span of 0.2 for mean 
value (blue box) and a normal variance that ranges from 0.5-0.8 (black line extended out the blue box). In contrast, NREL has a more regular shape with a span of 0.1 for mean value and a normal variance range of $0.15-0.3$, which are significantly smaller. It is extremely difficult to compare predictions of building energy consumption if the energy pattern has highly varying patterns driven by stochastic occupancy behavior and equipment usage levels. Hence, the fire station building predictions are sensitive to building size (i.e., small), building age (i.e., old) and building usage (i.e., not a normal office but an emergency center). The forecast model application for comparison purposes must recognize these fundamental differences in the building character and usage.

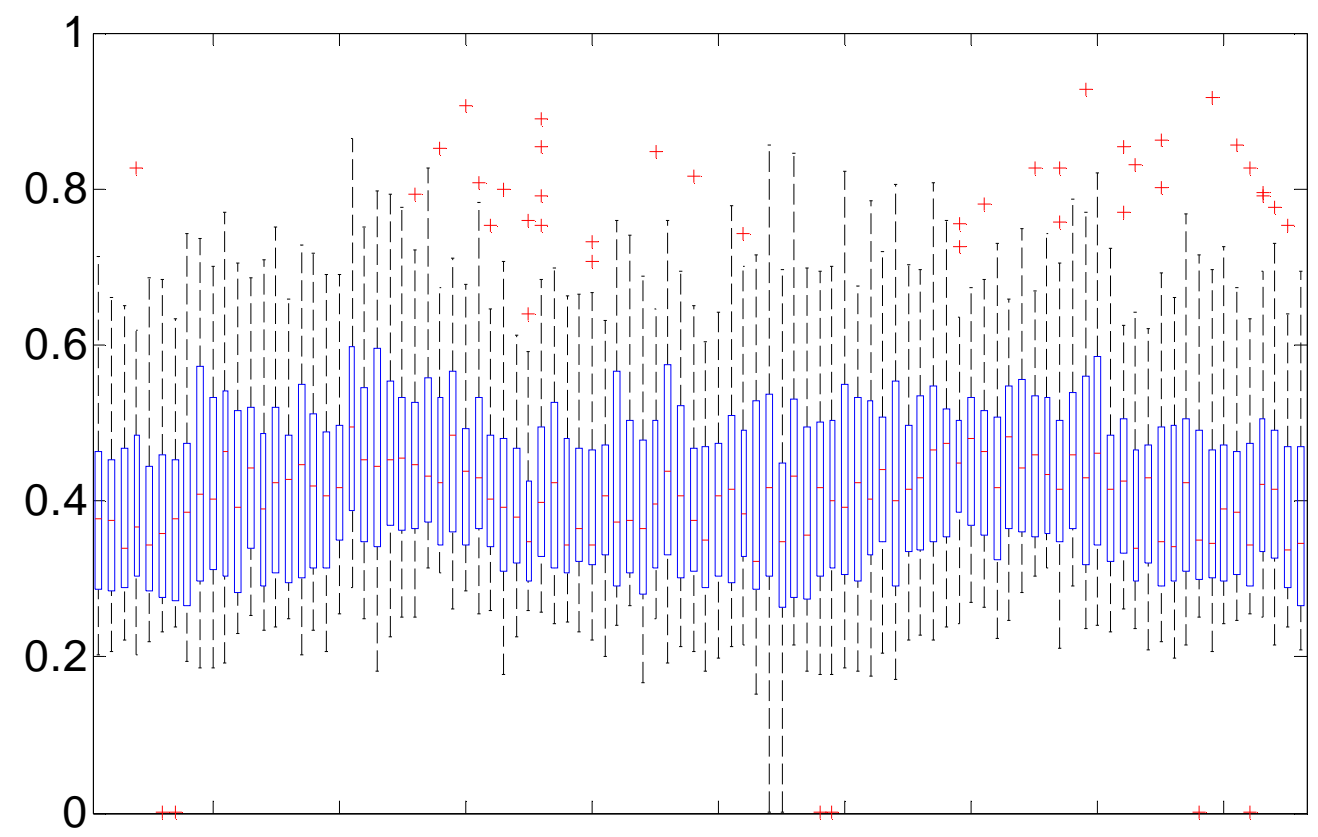




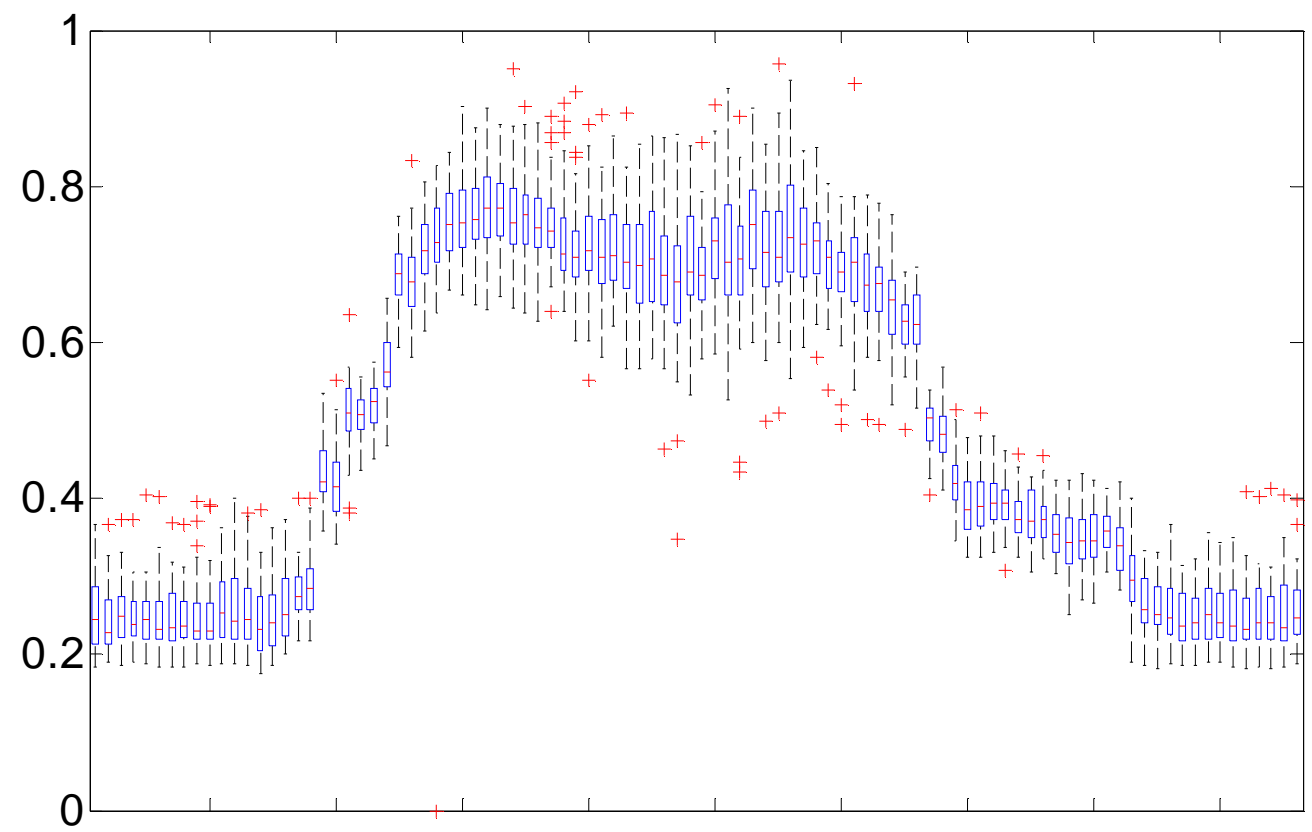

Figure 38: The fire station variance compared to NREL in a normalized score for 15 minute resolution

\subsubsection{Fort Sam Houston Library}

The Fort Sam Houston Library load forecasting demonstration period spanned from 6/18/2016 to 7/18/2016 (Table 6). The main matrix to evaluate the forecasting performances are calculated using MAPE. However competitive MAPE results in this case study are not expected because the small building load values at night provide very large MAPE errors.

Table 5: Fort Sam Houston Library Load Forecasting--MAPE Statistics

\begin{tabular}{c|c|c|c}
\hline Fort Sam & ANN & SVR & $\begin{array}{c}\text { Hybrid (SVR + } \\
\text { Persistent) }\end{array}$ \\
\hline 15-min ahead & $20.58 \%$ & $19.21 \%$ & $18.33 \%$ \\
\hline 1-hour ahead & $27.10 \%$ & $24.77 \%$ & $21.52 \%$ \\
\hline 24-hour ahead & $28.24 \%$ & $26.13 \%$ & - \\
\hline 36-hour ahead & $29.41 \%$ & $27.70 \%$ & - \\
\hline
\end{tabular}

The energy consumption pattern of the Fort Sam Library is comparable to the NREL buildings (Figure 39) with the performance notably closer to the RSF 2 building. This pattern is a result of minimum and maximum energy consumption peaks that are fairly regular but with different magnitudes than the NREL 
buildings. The regular spikes in energy consumption occurring in the morning and evening result from large equipment "on' and "off" periods tied directly to the operation schedule or feedback from the system providers and are very difficult to predict.

In conclusion, the load forecasting capability demonstrated as part of the INTEGRATE effort provides new innovative solutions that enhance our ability to predict building energy consumption in time scales of significant interest to building operators, utilities and distributed energy providers. The capability extends well beyond traditional linear regression or persistent approaches and embodies two artificial machinelearning methods, Artificial Neural Network and Support Vector Machine that are specifically designed for on-line forecasting. These methods were selected among many other popular black box approaches, including Gaussian Mixture Model, Gaussian Process Approach, and Autoregressive-Moving-Average Model. These models are specifically divided into two parallel stages: online forecasting and offline optimization. The online predictions are forecasted by the prefixed parameters which guarantee fast simulation speed within 1 minute and allow a total of 5 minute limit forecasts publishing from 15-minute ahead to 36-hour ahead window. The prefixed parameters are constantly updated once the operator initiates the offline optimization that utilizes cross validation to verify model stability according to a grid

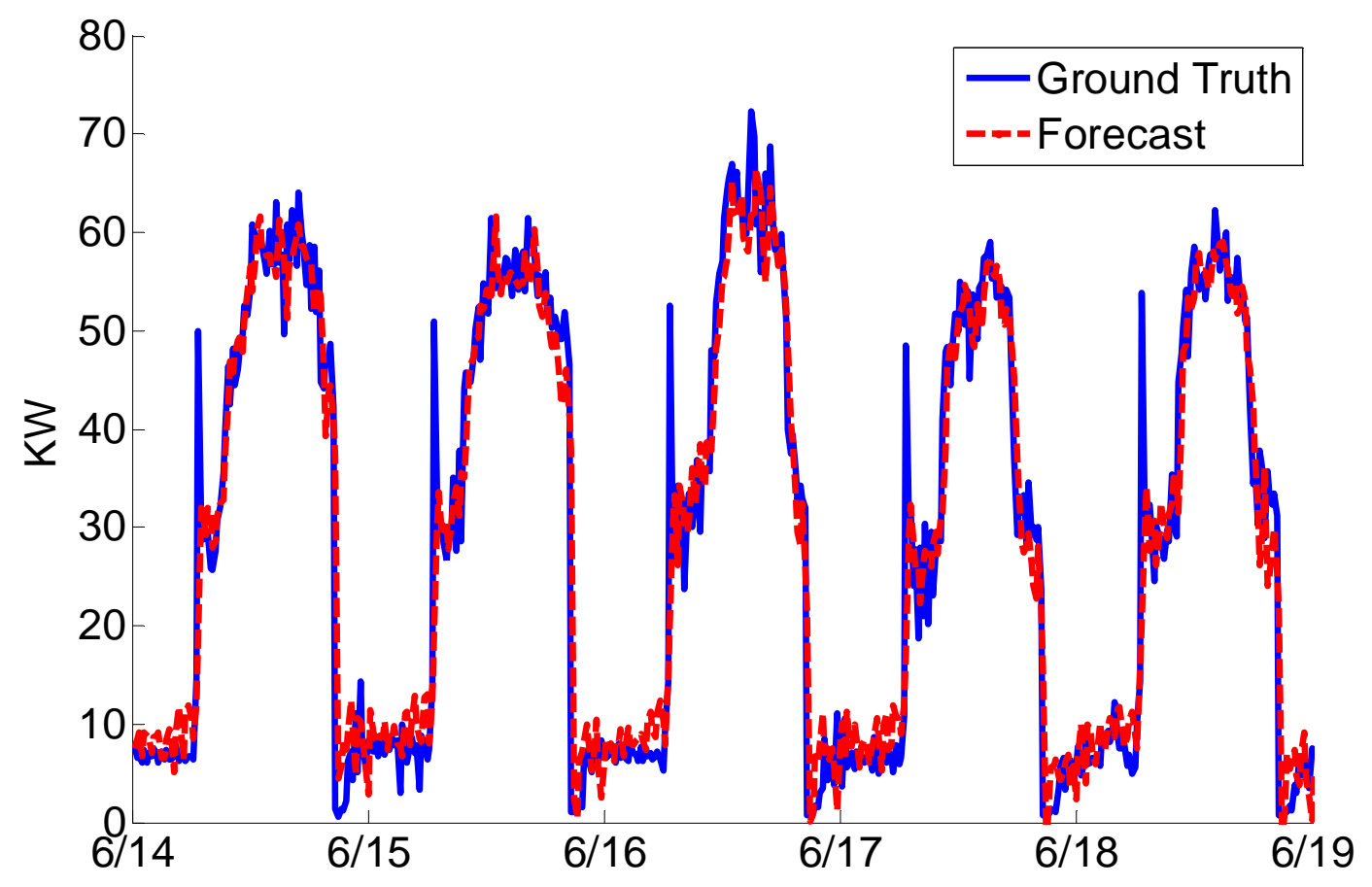

Figure 39: Comparison of measured and forecasted energy consumption for the Fort Sam Houston Library

\subsubsection{Day-Ahead Solar PV Production Forecasting}

The results, challenges and opportunities associated with the day-ahead solar PV forecasting varied between the three different test locations at NREL, McAlpine and Fort Sam Houston, often for different reasons. Clearly, site control and access, site operational and information technology procedures and 
protocols and the differences in the mission of each facility had a significant impact on the results. A summary of results for each site is presented for 15-minute ahead, 1-hour ahead, 24-hour ahead and 36hour ahead increments using 15-minute interval data.

\subsubsection{NREL Solar Forecast}

In general, there are two types of inputs for PV energy output forecasting: exogenous inputs from the meteorological forecasts and endogenous inputs from the direct system production outputs. The meteorological forecasts using solar irradiance and related parameters have been studied extensively. However, even with the cloud graph from synchronous meteorological satellites, large variability in key parameters, namely the diffuse component from the sky hemisphere, makes solar irradiance much less reliable than temperature as a forecast input. In this study, we assume two cases: one is that the inputs can be as accurate as possible from the solar forecast, and the other is solely based on non-solar inputs. For the NREL RSF building PV day-ahead forecast it is a challenging task in part because of the variability and limitations of the input data.

The NREL RSF building PV forecasting effort collected data for four months to produce forecasts for both RSF buildings. The data collection period extended from 9/1/2015 to 12/31/2015. The results of the forecast predictions were calculated using a Normalized Mean Absolute Error (NMAE), as follows.

$$
N M A E=\frac{1}{N} \sum_{i=1}^{N}\left|y_{i}-y_{p}\right| / C
$$

where $y_{i}$ is the actual energy consumption, $y_{p}$ is the predicted energy consumption, $\mathrm{N}$ is the total number of predictions, and $\mathrm{C}$ is the capacity of the system. The smaller the NMAE calculated, the better the forecast.

All prediction performances are summarized in Tables 7 and 8 .

Table 6: NREL Short Term PV Forecasting NMAE Statistics

\begin{tabular}{c|c|c|c|c|c}
\hline RSF 1 & ANN & SVR & $\begin{array}{c}\text { SVR (without } \\
\text { solar) }\end{array}$ & $\begin{array}{c}\text { Hybrid (SVR + } \\
\text { Persistent) }\end{array}$ & $\begin{array}{c}\text { Hybrid (without } \\
\text { solar) }\end{array}$ \\
\hline 15-min ahead & $7.34 \%$ & $6.80 \%$ & $7.91 \%$ & $6.46 \%$ & $7.46 \%$ \\
\hline 1-hour ahead & $11.16 \%$ & $10.12 \%$ & $10.72 \%$ & $8.61 \%$ & $9.55 \%$ \\
\hline RSF 2 & ANN & SVR & SVR (without & Hybrid (SVR + & Hybrid (without \\
\hline 15-min ahead & $8.60 \%$ & $7.09 \%$ & $8.38 \%$ & Persistent) & solar) \\
\hline 1-hour ahead & $10.41 \%$ & $10.18 \%$ & $10.24 \%$ & $9.63 \%$ & $8.14 \%$ \\
\hline
\end{tabular}

Table 7: NREL Long Term PV Forecasting NMAE Statistics 


\begin{tabular}{c|c|c|c}
\hline RSF 1 & ANN & SVR & $\begin{array}{c}\text { SVR (without } \\
\text { solar) }\end{array}$ \\
\hline 24-hour ahead & $16.24 \%$ & $15.91 \%$ & $16.39 \%$ \\
\hline 36-hour ahead & $21.34 \%$ & $21.55 \%$ & $22.16 \%$ \\
\hline RSF 2 & ANN & SVR & $\begin{array}{c}\text { SVR (without } \\
\text { solar) }\end{array}$ \\
\hline 24-hour ahead & $15.52 \%$ & $15.88 \%$ & $16.61 \%$ \\
\hline 36-hour ahead & $21.24 \%$ & $22.73 \%$ & $22.84 \%$ \\
\hline
\end{tabular}

The results from both RSF1 and RSF2 demonstrate that the forecast model accuracy is greatly influenced by the quality and nature of the available input data for calibrating the model outputs. The temporal variability and quality of the data feeds becomes even more critical during those times of the year where local weather conditions are highly variable and extensive periods of time exist with wind, rain and clouds, hence the quality of the forecasts reflect real-world variability in the local weather. Where weather conditions appear stable for extended periods, either sunny or partly cloudy (Figure 40 and 41) forecast predictions and measured outputs are very consistent. However, as discussed later in the report, for those conditions where the weather is highly variable over time frames of 24- and 36-hour periods, which is "real-world" in many locations, the NMAE increases significantly.

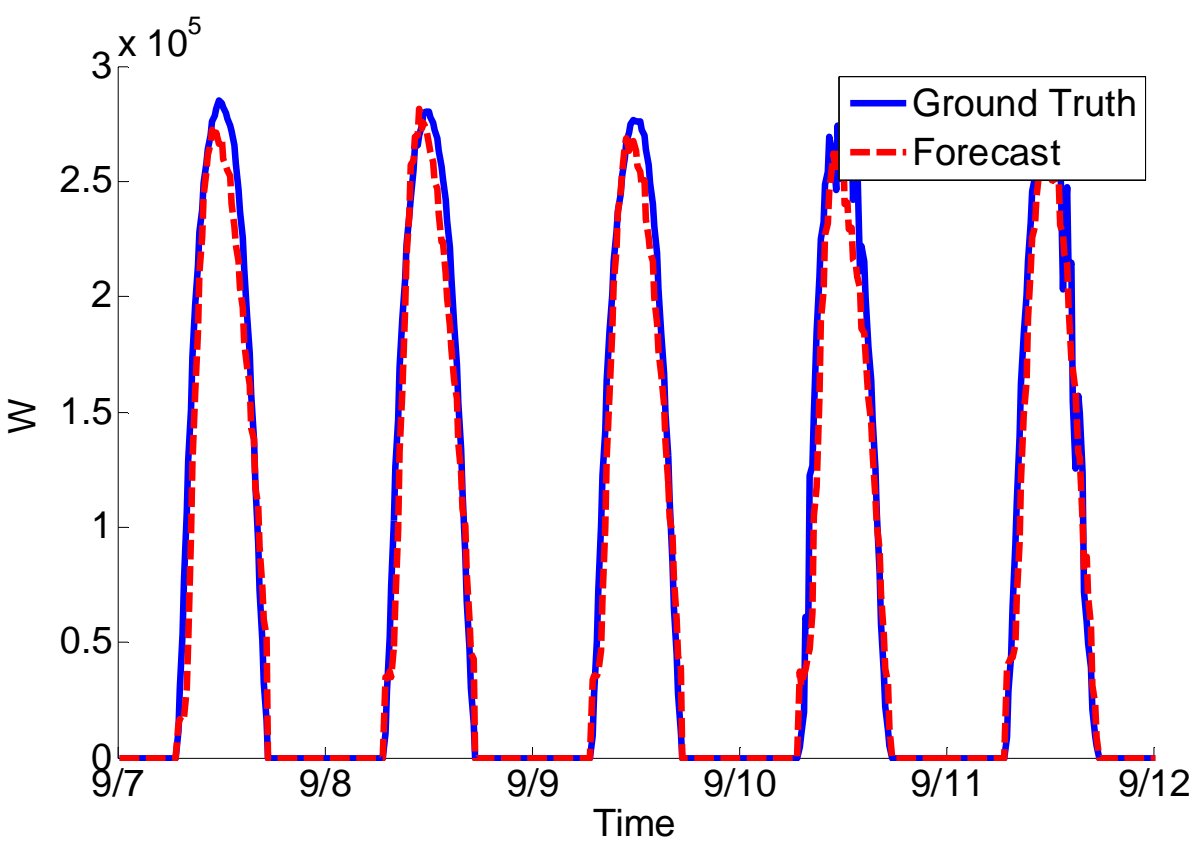

Figure 40: Solar Forecasts for RSF1 over a sunny period 


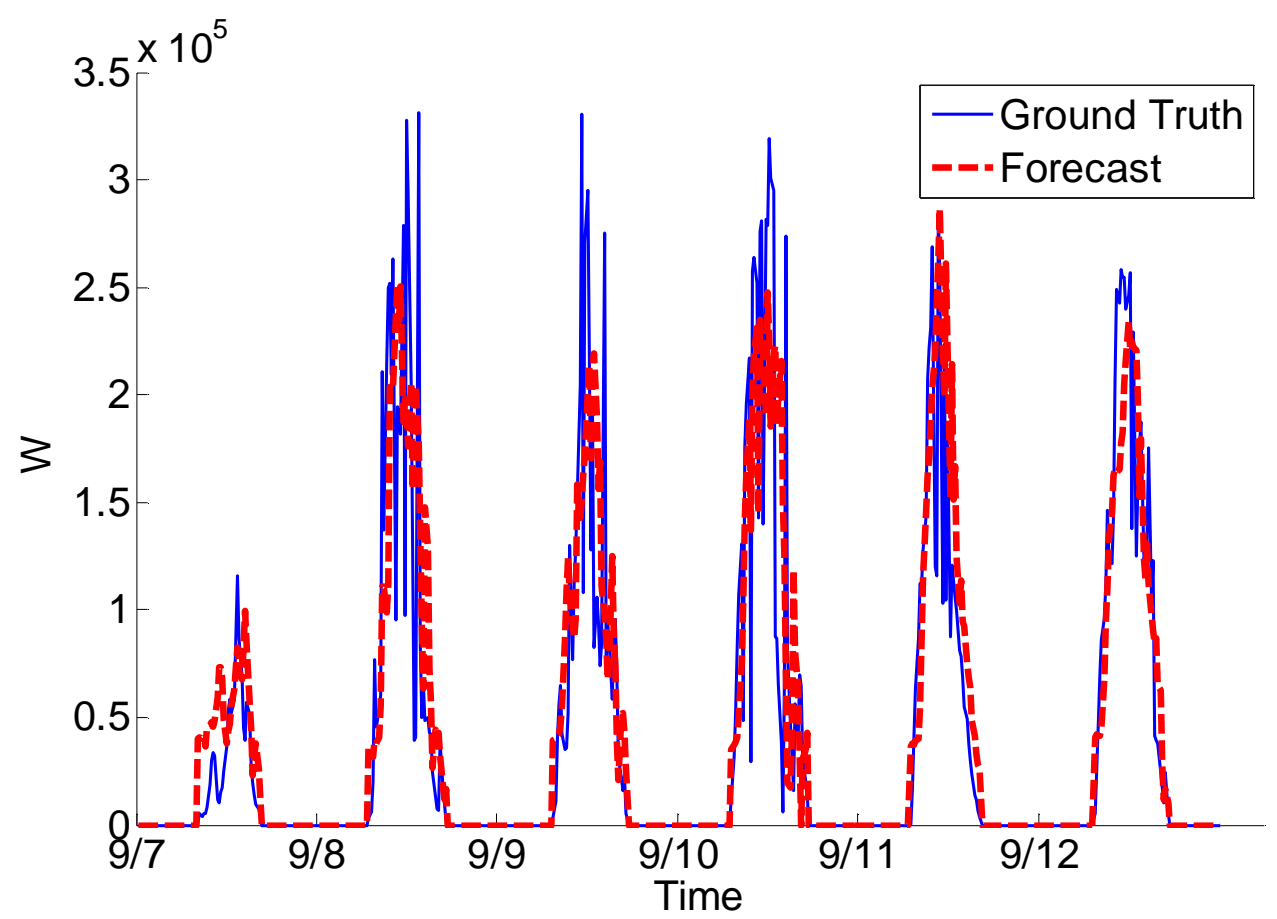

Figure 41: Solar Forecasts for RSF2 over a partly cloudy period

\subsubsection{McAlpine PV Forecast}

The McAlpine fire station demonstration period lasted nearly 2 months extending from 3/3/2014 to $5 / 14 / 2014$. The results of the short term, intra-hour forecasts (Table 9) were calculated using NMAE.

Table 8: Short term forecasting NMAE statistics

\begin{tabular}{c|c|c|c|c|c}
\hline $\begin{array}{c}\text { Fire Station's } \\
\text { PV }\end{array}$ & ANN & SVR & $\begin{array}{c}\text { SVR (without } \\
\text { solar) }\end{array}$ & $\begin{array}{c}\text { Hybrid (SVR } \\
\text { + Persistent) }\end{array}$ & $\begin{array}{c}\text { Hybrid } \\
\text { (without solar) }\end{array}$ \\
\hline 15-min ahead & $12.84 \%$ & $12.37 \%$ & $11.40 \%$ & $10.72 \%$ & $10.87 \%$ \\
\hline 1-hour ahead & $14.63 \%$ & $14.57 \%$ & $15.42 \%$ & $13.69 \%$ & $13.95 \%$ \\
\hline
\end{tabular}

From the NMAE results, the PV system forecasts at McAlpine are much less accurate than those calculated for either NREL building. The evaluation period at McAlpine was influenced significantly by local variations in weather with variable winds and clouds, creating a highly variable production cycle over the course of the performance period (Figure 42). The variable weather conditions dampen the model performance because of the extreme peak values of solar irradiance and significantly distinct daily patterns. Examination of the 24-hour results shows similar results (Table 10). To accommodate these 
highly variable weather conditions, detailed weather models may be required as additional inputs to the forecast methodologies to improve the approach's predictive capability of PV generation.

Table 9: Long Term PV Forecasting NMAE Statistics

\begin{tabular}{c|c|c|c}
\hline $\begin{array}{c}\text { Fire Station's } \\
\text { PV }\end{array}$ & ANN & SVR & $\begin{array}{c}\text { SVR (without } \\
\text { solar) }\end{array}$ \\
\hline 24-hour ahead & $22.92 \%$ & $22.86 \%$ & $23.64 \%$ \\
\hline 36-hour ahead & $26.33 \%$ & $25.47 \%$ & $26.38 \%$ \\
\hline
\end{tabular}

\subsubsection{Fort Sam Houston}

The Fort Sam Houston forecasts were calculated using only non-solar related meteorological forecasts (Table 11) because of the lack of solar irradiance measurements and predictions. The PV forecast results were assessed over the period from 6/18/2016 to 7/18/2016. The PV solar forecast results (Table 11) were again influenced by the highly variable weather conditions influenced by variability in cloud cover.

Table 10: Fort Sam Houston PV Forecasting—NMAE Statistics

\begin{tabular}{c|c|c|c|c|c}
\hline Fort Sam & ANN & SVR & $\begin{array}{c}\text { Hybrid (SVR } \\
\text { + Persistent) }\end{array}$ & $\begin{array}{c}\text { SVR } \\
\text { (Sunny } \\
\text { days) }\end{array}$ & $\begin{array}{c}\text { Hybrid } \\
\text { (Sunny } \\
\text { days) }\end{array}$ \\
\hline 15-min ahead & $8.05 \%$ & $8.14 \%$ & $7.62 \%$ & $7.23 \%$ & $7.12 \%$ \\
\hline 1-hour ahead & $9.07 \%$ & $9.13 \%$ & $8.32 \%$ & $7.78 \%$ & $7.69 \%$ \\
\hline 24-hour ahead & $14.95 \%$ & $15.23 \%$ & - & $14.85 \%$ & - \\
\hline 36-hour ahead & $16.03 \%$ & $16.61 \%$ & - & $15.19 \%$ & - \\
\hline
\end{tabular}




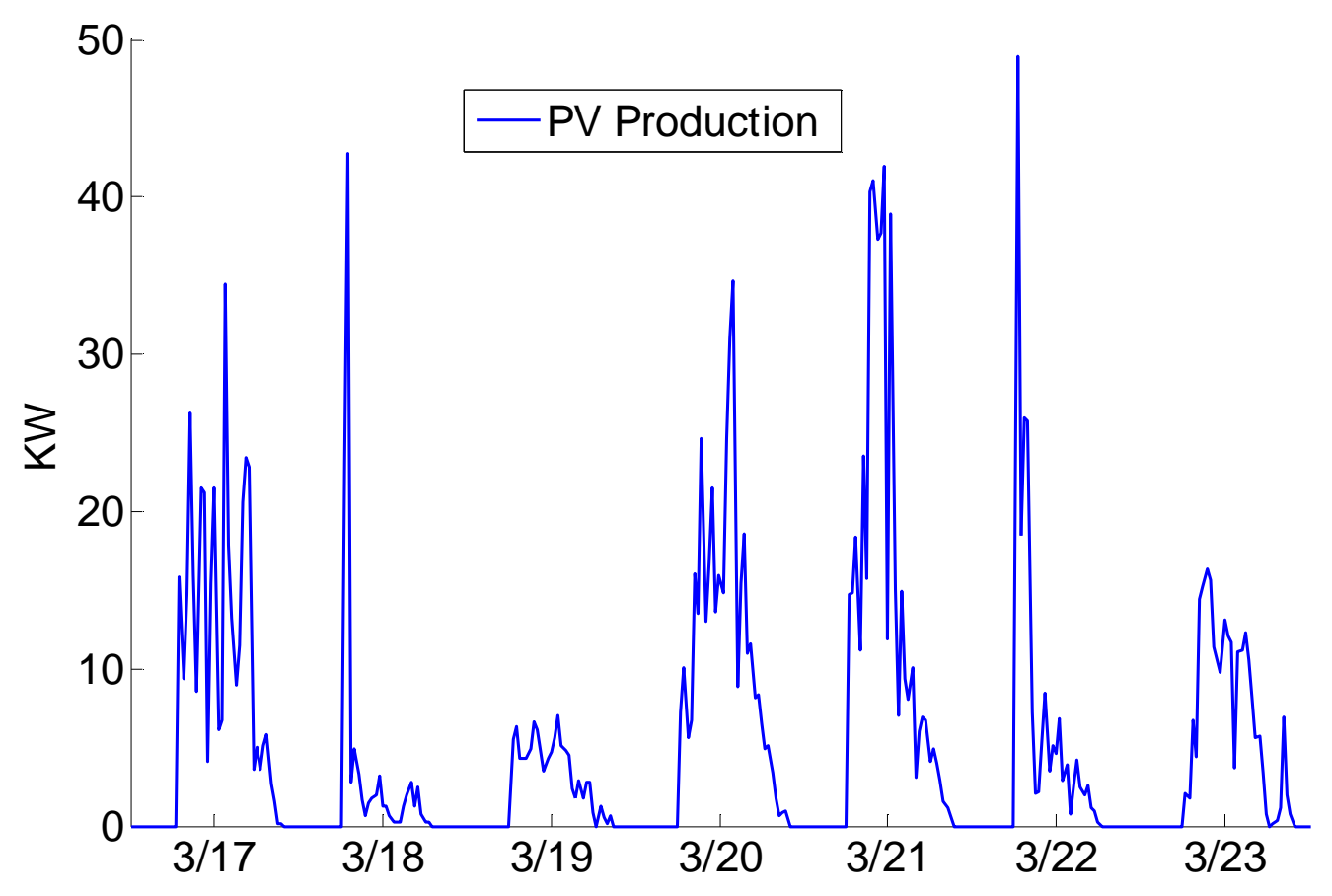

Figure 42: Fort Sam Houston PV Forecasting during sunny days

\subsection{Integration with OpenFMB}

In parallel to the forecasting activities, real-time data management (Figure 43) is needed to provide near real-time forecasting of building load and solar PV generation for micro-grid environments. The challenges of real-time data management are:

- Synchronization: getting data where it needs to be and when it needs to be there

- Expandability: capacity to expand the system to meet real world challenges (e.g., demand for new forecasts)

- Portability: producing both cloud and local data exchange API's and maintaining interoperability.

OpenFMB is a framework for distributed intelligent nodes interacting with each other through loosely coupled, peer-to-peer messaging for fielded devices and systems at the grid edge. The OpenFMB framework provides a specification for power systems field devices to leverage a non-proprietary and standards-based reference architecture consisting of internet protocol (IP) networking and Internet of Things (IoT) messaging protocols. OpenFMB can act both as a means to pass data and as a triggering function, removing some of the difficulties with synchronization (Figure 44).

OpenFMB facilitates an expandable framework (Figure 45) because the design is hierarchical in structure. Subscribers can access information without requiring complete code rewrites and can decide which 
information they want to collect from all of the information offered by the broker. Providers also benefit as the structure readily accommodates new inputs with limited effort.

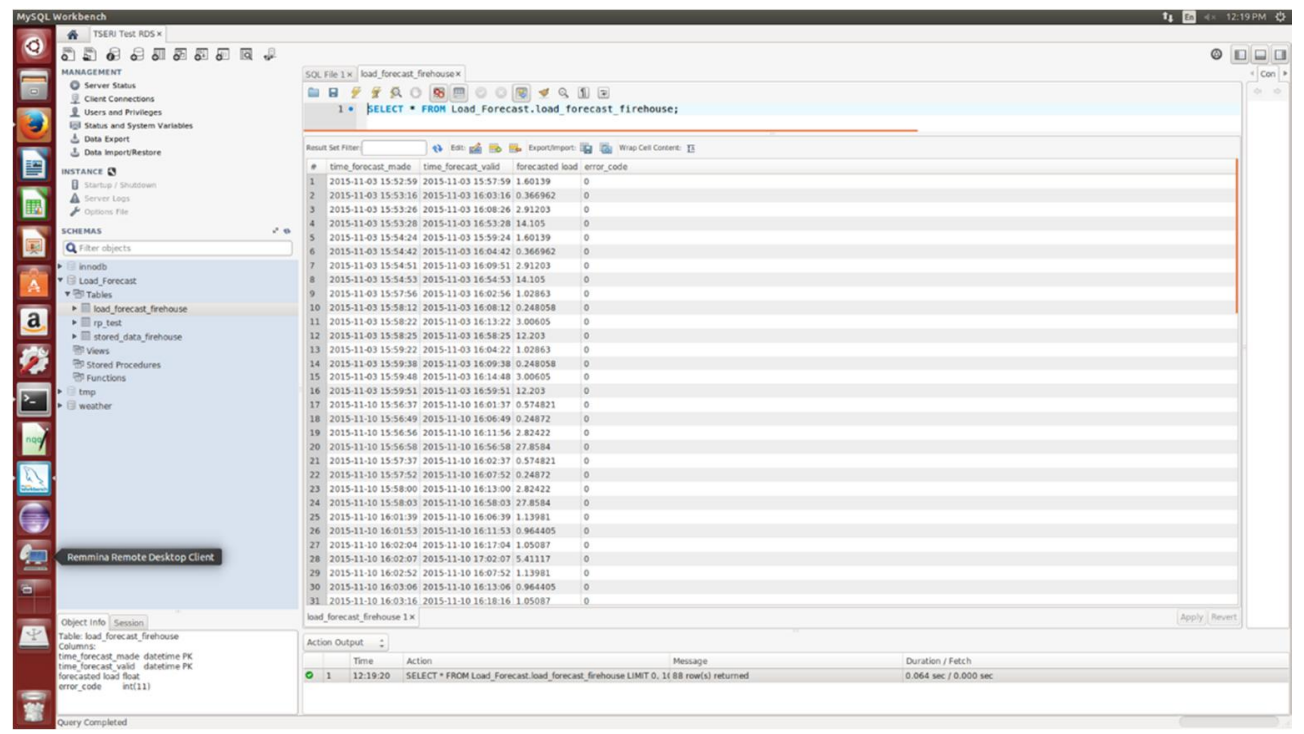

Figure 43: Capture of the administration tool for the databases

The system includes an MQTT (Message Que Telemetry Transport) communication layer, a Python based historian that translates MQTT messages into a MySQL database, real-time publisher of data, a weather information gathering application to scrape online weather content, and subscriber framework for building applications that leverage the published data in the MQTT broker. Additionally, the load and solar forecasting applications create outputs that are in the OpenFMB XSD scheme for utilization of XML subscriber applications. The system is deployed in a cloud environment and on a private network.

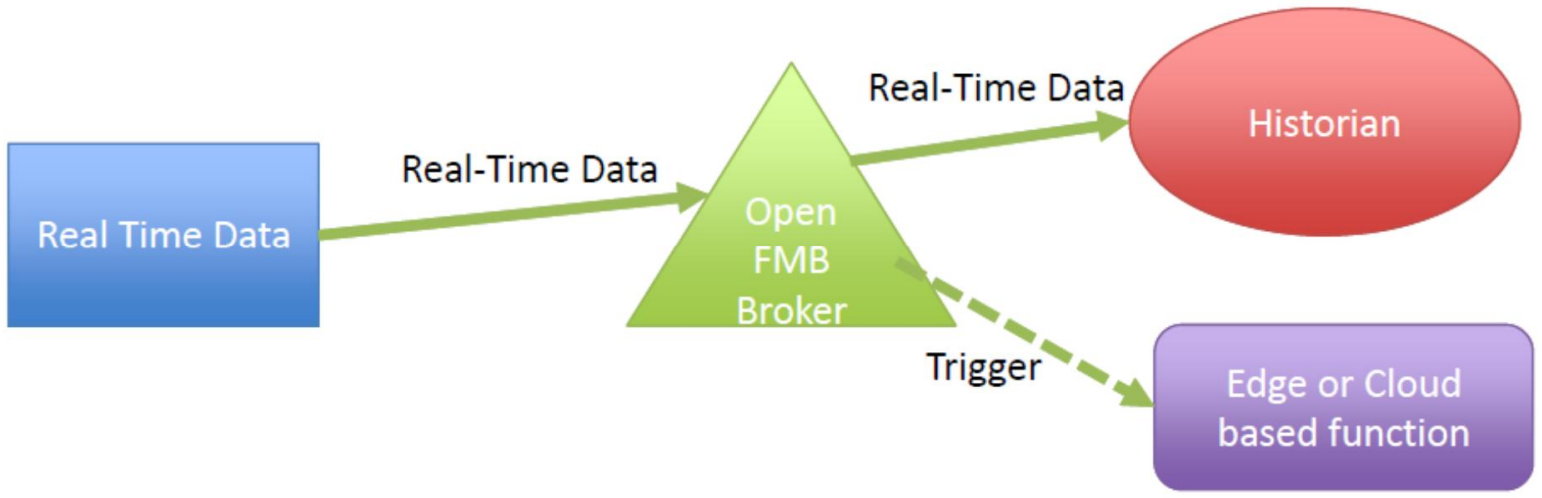

Figure 44: A synchronization scheme implemented through OpenFMB 


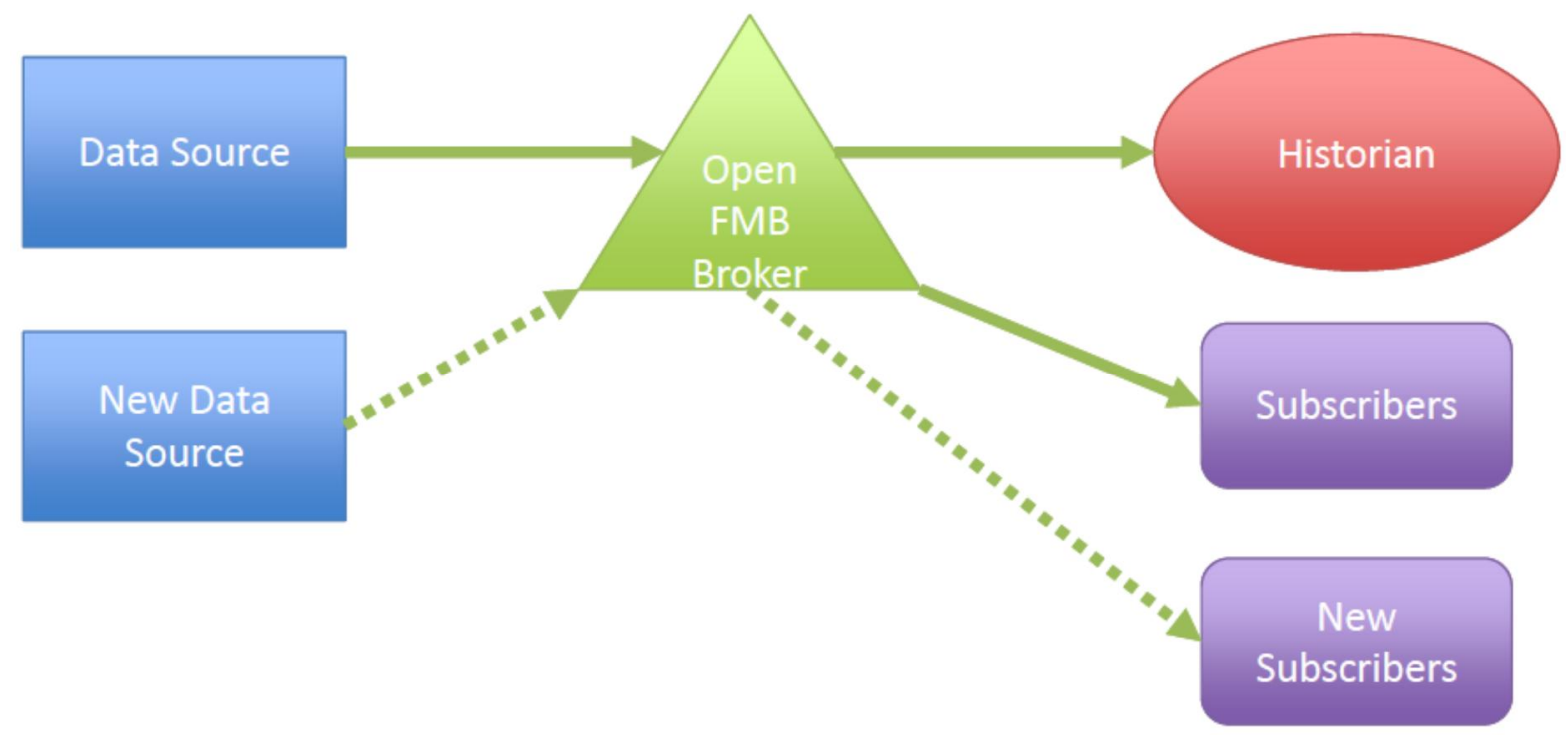

Figure 45 Scheme illustrating OpenFMB expandability

MQTT is a centralized, publish/subscribe framework organized by a centralized broker and "messages" anything from strings and integers to data and files- are published on specific topics (Figure 46). These topics follow a file system like hierarchy:/topic/subtopic/sub-subtopic. Messages pass through the following process:

1 Publisher publishes a message on a topic. That message arrives to the broker

2 The broker passes the message to all of the subscribers who are subscribed to that topic

3 The subscribers receive the message

4 Lastly, qualities of service statuses are resolved

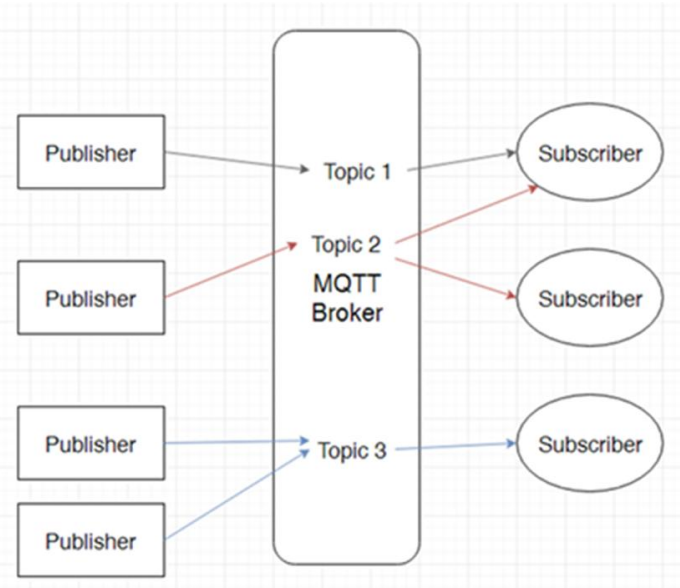

Figure 46: MQTT Communications Scheme 
To set up the MQTT Broker and the historian, several installation files have been created for automated faster deployment. The MQTT Broker is the simplest component of the system to install but also the most important. There are two possible modes of installation: a mode where the broker and historian reside on the same machine and a mode where the broker and historian reside on separate machines.

MQTT is also a publish/subscribe framework. The broker provides a file system-like topic hierarchy that publishers publish into and subscribers subscribe to. How publishers and subscribers interact with the broker is covered in detail based on the MQTT Python Library. For this project, the broker is relatively passive; publishers and subscribers define our system, but it is possible for the broker to be more active with security and backup or clustered brokers.

A demonstration of one of our forecasts coming online is illustrated in Figure 47. It is connected to the broker and waits until the data is received, and once the data is received, the forecast is produced and published.

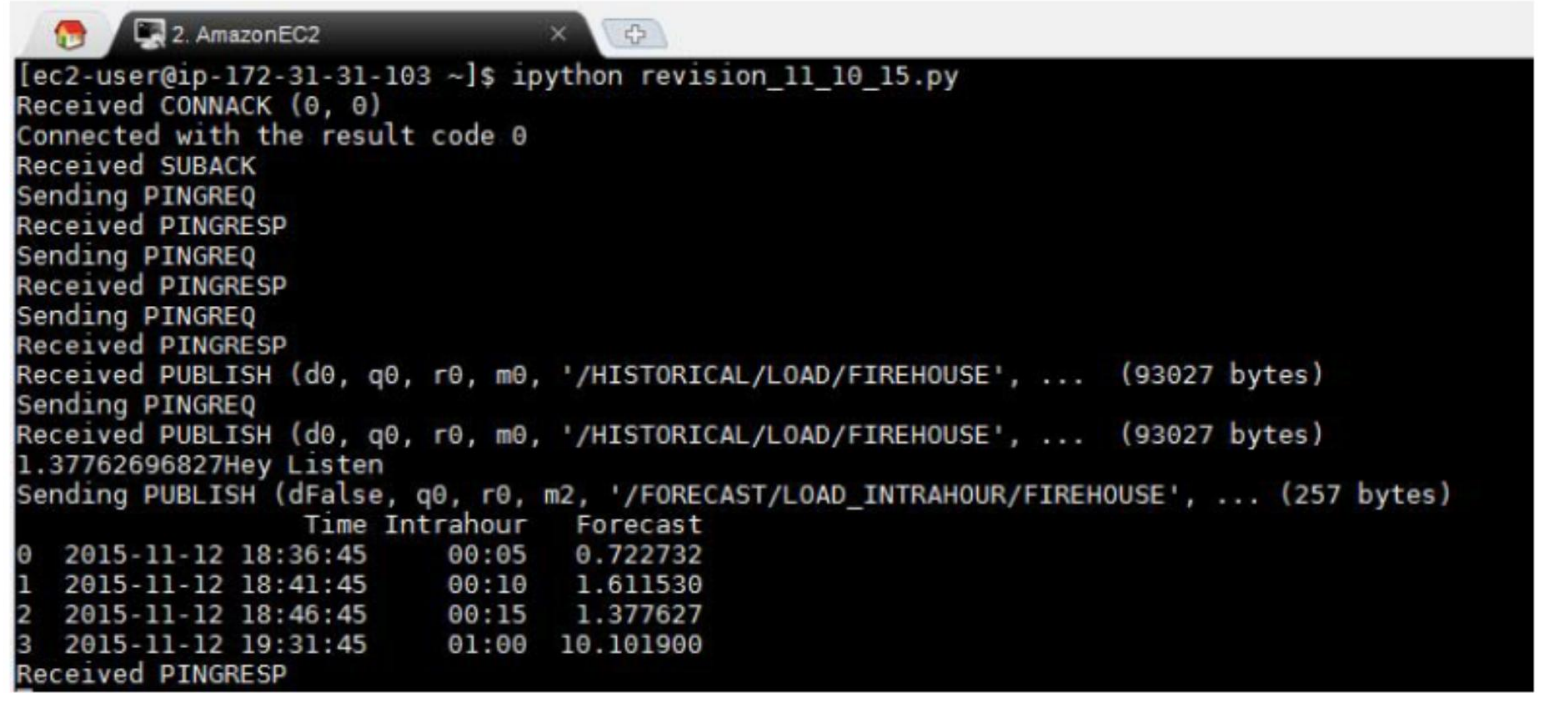

Figure 47: Screen capture of the OpenFMB process when input data is provided and forecasting is published

Quality of service (QoS) is used and defined in MQTT to indicate the level of confidence the message has been received. The lowest level, 0 , means that a publisher sends out a message with no expectation that it will receive any confirmation that the message was sent. The next quality of service is 1 , which is known as "at least once." The publisher will continually resend a message until it gets a confirmation from the broker that the message has been received (Called a Puback, short for "publish acknowledgement" packet). A QoS of 2 is known as "only once" in this case there is a multipart handshake process. The specific implementation means that each device at a QoS of 2 will receive each message only once and 
more specifically ignore rebroadcasts of the same message. Note that subscribers and publishers can have different QoS levels (however, the subscribers' QoS can never be higher than the publishers').

\subsection{Description of the Online Deployment Platform}

The deployment in the MGMS is illustrated in Figure 48. The deployment is based on inputs of two different solar forecasting hardware applications (day-ahead and intra-hour), running on Python codes and the capture of different weather parameters. The hard-kernel produces the forecast outputs that are published by a MQTT publisher/subscriber framework in the IoT cloud. The load forecasting application acts as MQTT subscriber of the solar forecasting publisher, but also use the MQTT framework to publish the load forecast. Finally, the Omnetric MGMS acts as subscriber of the solar and load forecasting subscribers to manage the smart grid.

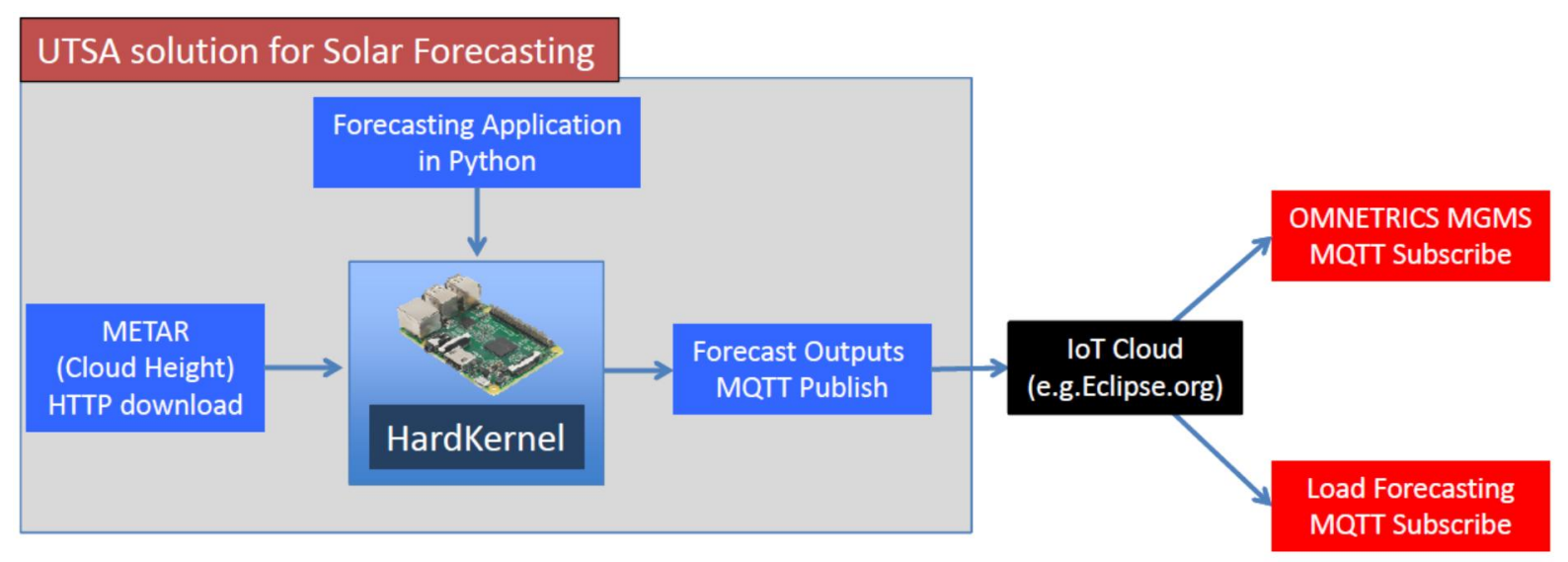

Figure 48: Scheme of the suggested deployment in the MGMS

\subsubsection{Load forecasting and day-ahead PV production forecasting}

The UTSA solution for load and solar forecasting has been tested in RUGGEDCOM VM by MQTT publishing (Figure 49). Data is collected each day for the entire 24-hour period at 10-15 seconds intervals. 


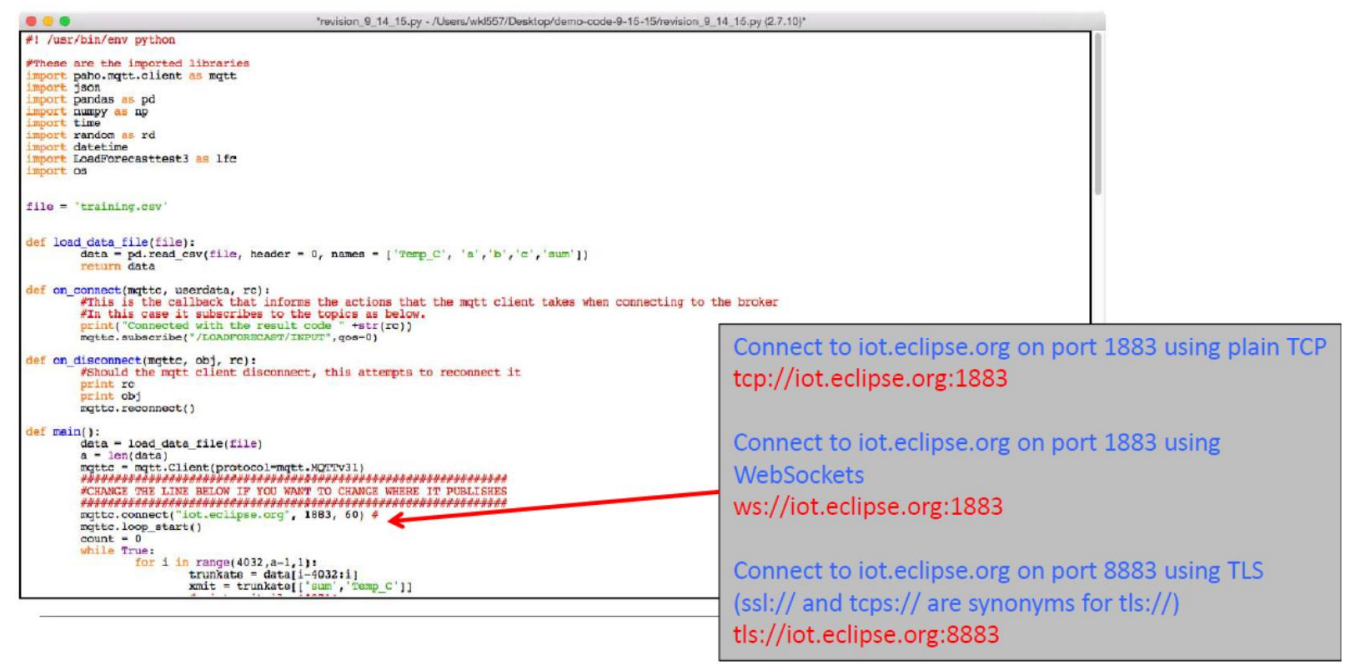

Figure 49: Screen capture showing an extract of the routines for forecasting

The forecasting tool setup (install.sh created) in RUGGEDCOM VM was successful (Figure 50)

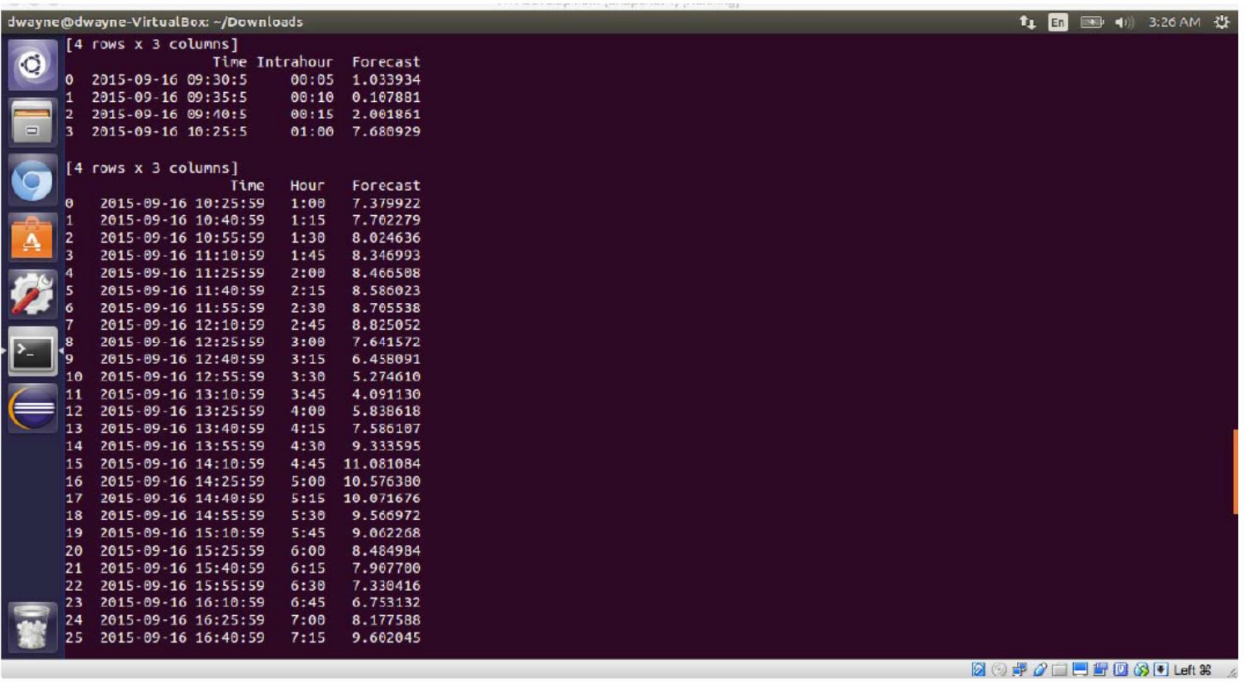

Figure 50: Screen capture showing how the forecasting tools are provided

\subsubsection{Sky Imager Intra-hour Forecasting at NREL}

The Sky Imager was located at the NREL facility to analyze the irradiance spikes based on cloud shading and its effect on a solar PV plant production. The sky-imager was located close to the $408 \mathrm{~kW}$ PV plant tested on the roof of the RSF2 building (Figure 51). The images where captured using the Fusion Technique, blocking the sun, masking images, orienting these images to the true North based on the sun trajectory, cloud segmentation, geometric transformation, cloud motion analysis, cloud shadow ray-trace analysis, and irradiance prediction for 5, 10 and 15 minutes. 


\section{OMNETRIC $_{\text {Group }}$}
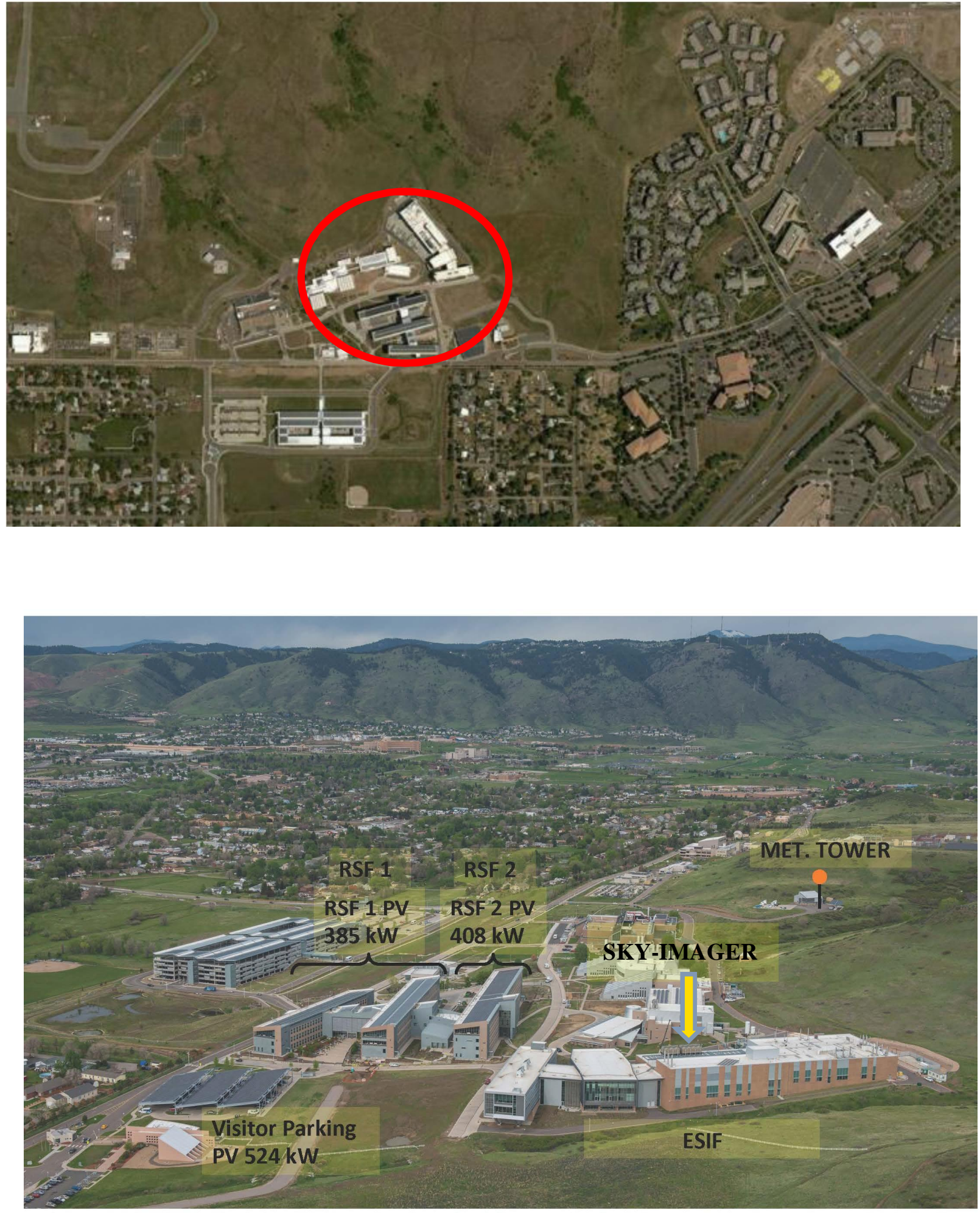

Figure 51: NREL site instrumentation for the sky imager, MET tower and PV plants 
The regions of interest were the cloud areas used to ray trace a "shading" onto the NREL facility (Figure 52) and their predicted movement over time. If there is a shadow forecasted in the PV plant, the irradiance observed by the panels will be much lower. If there are no shadows forecasted, then the PV panels should expect the irradiance profile that is forecasted for the day under clear sky conditions.

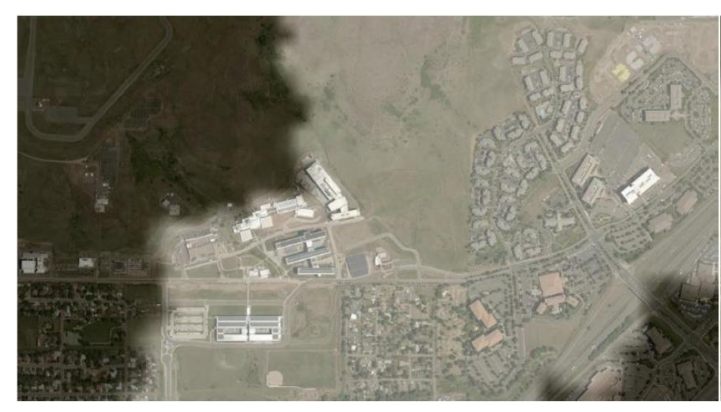

(a)

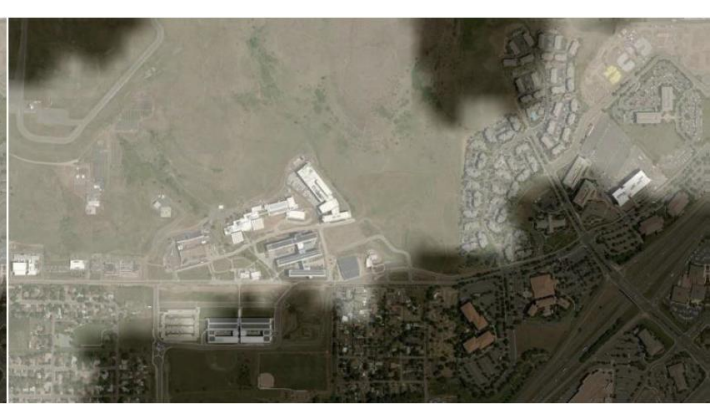

(b)

Figure 52: (a) Current cloud cover at NREL; and (b) cloud cover over NREL 5-min ahead. Note: clear sky appears bright and shading appears black

The graphs in Figure 53 show the measured direct solar irradiation (DSI) for five different days and the associated variance in irradiance profiles (i.e., shadow/no shadow). The measured DSI values where recorded using NREL's CHP1 Pyrheliometer. Also included in these graphs are the forecasted irradiance spikes generated by the methodology described previously. The images use data collected daily between 10 am and $4 \mathrm{pm}$ to bound the period of time where the greatest variability in irradiance is likely to occur. 


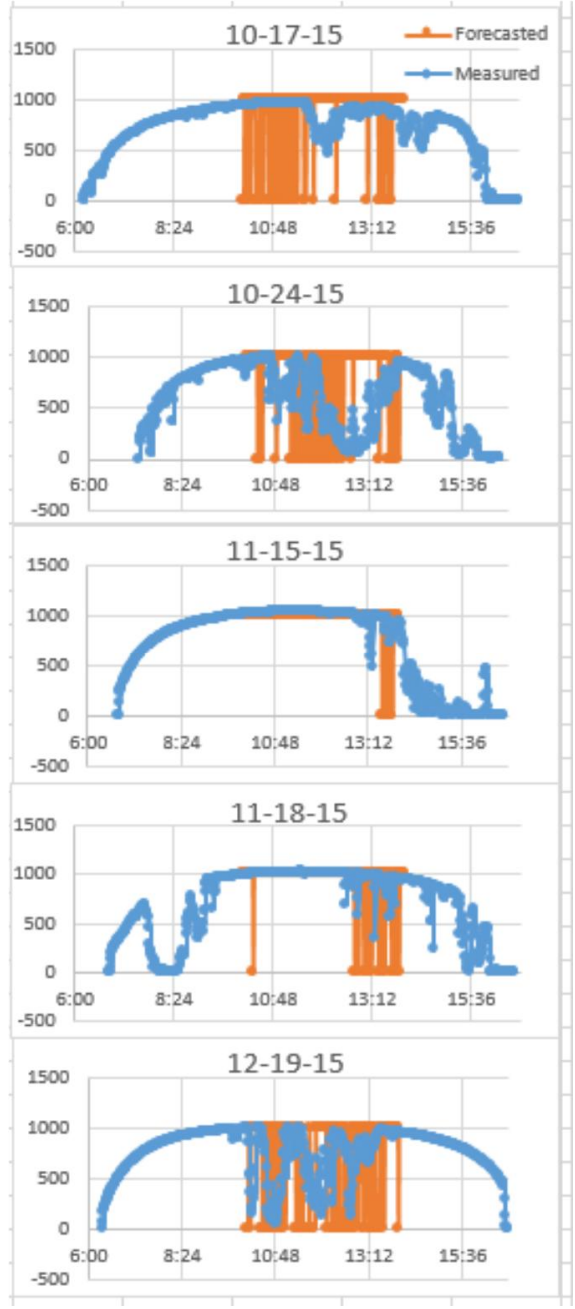

Figure 53: Direct Solar Irradiance values for five days at NREL. Blue is the measured value during daylight hours and orange is the predicted cloud/no cloud value during the hours $10 \mathrm{am}-4 \mathrm{pm}$

The mean absolute percentage error (MAPE) calculated for these days is summarized in Table 12. The results highlight how well the methodology performs under various conditions. As anticipated, the days dominated by clear skies with limited clouds are associated with the lowest MAPE.

Table 11: Mean Absolute Percentage Error (MAPE) of solar irradiance for the days specified

\begin{tabular}{|l|l|l|l|l|l|}
\hline Date: & $\mathbf{1 0 - 1 7 - 2 0 1 5}$ & $\mathbf{1 0 - 2 4 - 2 0 1 5}$ & $\mathbf{1 1 - 1 5 - 2 0 1 5}$ & $\mathbf{1 1 - 1 8 - 2 0 1 5}$ & $\mathbf{1 2 - 1 9 - 2 0 1 5}$ \\
\hline 5 -min forecast & $18.51 \%$ & $37.23 \%$ & $3.61 \%$ & $14.46 \%$ & $34.89 \%$ \\
\hline
\end{tabular}


Many lessons were learning during the deployment at NREL. For example, finding optimal versions of Linux, python, and OpenCV, for a particular single board computer and providing effective weatherproof enclosures, heater/fan assemblies, and heat sinks. Another important challenge was developing an efficient interface between the Sky Imager and the Micro-Grid Management System (MGMS).

\subsection{Sky Imager Deployment at NREL}

The UTSA Sky-Imager was deployed on the ESIF roof at NREL (Figures 54 and 55) using a tri-pod system connected to the MGMS via VLAN for testing and evaluation purposes. The Sky Imager was integrated using OpenFMB into the MGMS with the objective of testing for the end user (utility or other party) a highly reliable supply of electricity from renewable resources, conventional resources and energy storage, configured in a microgrid.

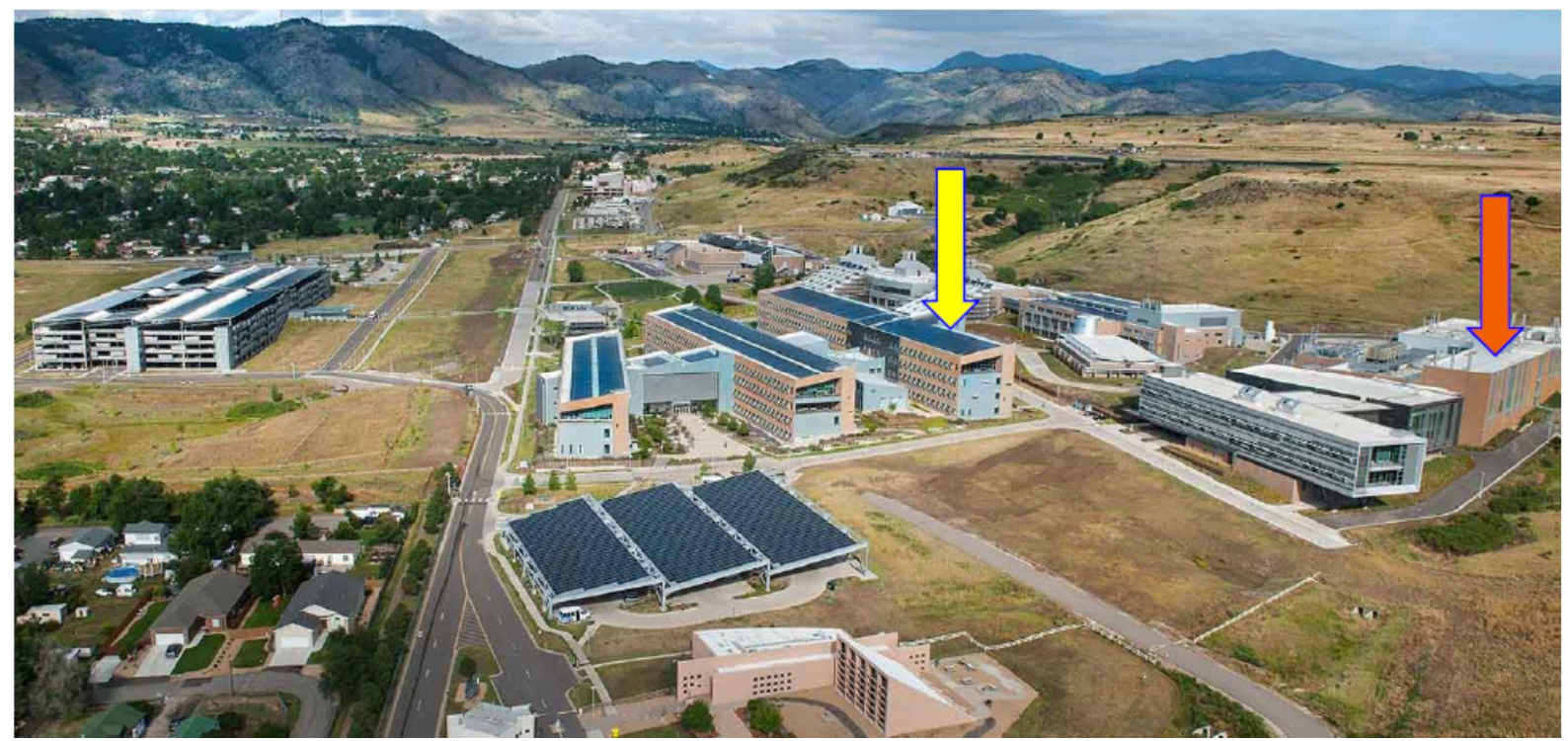

Figure 54: Location of Sky Imager (orange arrow) and the $408 \mathrm{PV}$ plant monitored (yellow arrow) at NREL 


\section{OMNETRIC $C_{\text {oroup }}$ \\ A Siemens \& Accenture Company}

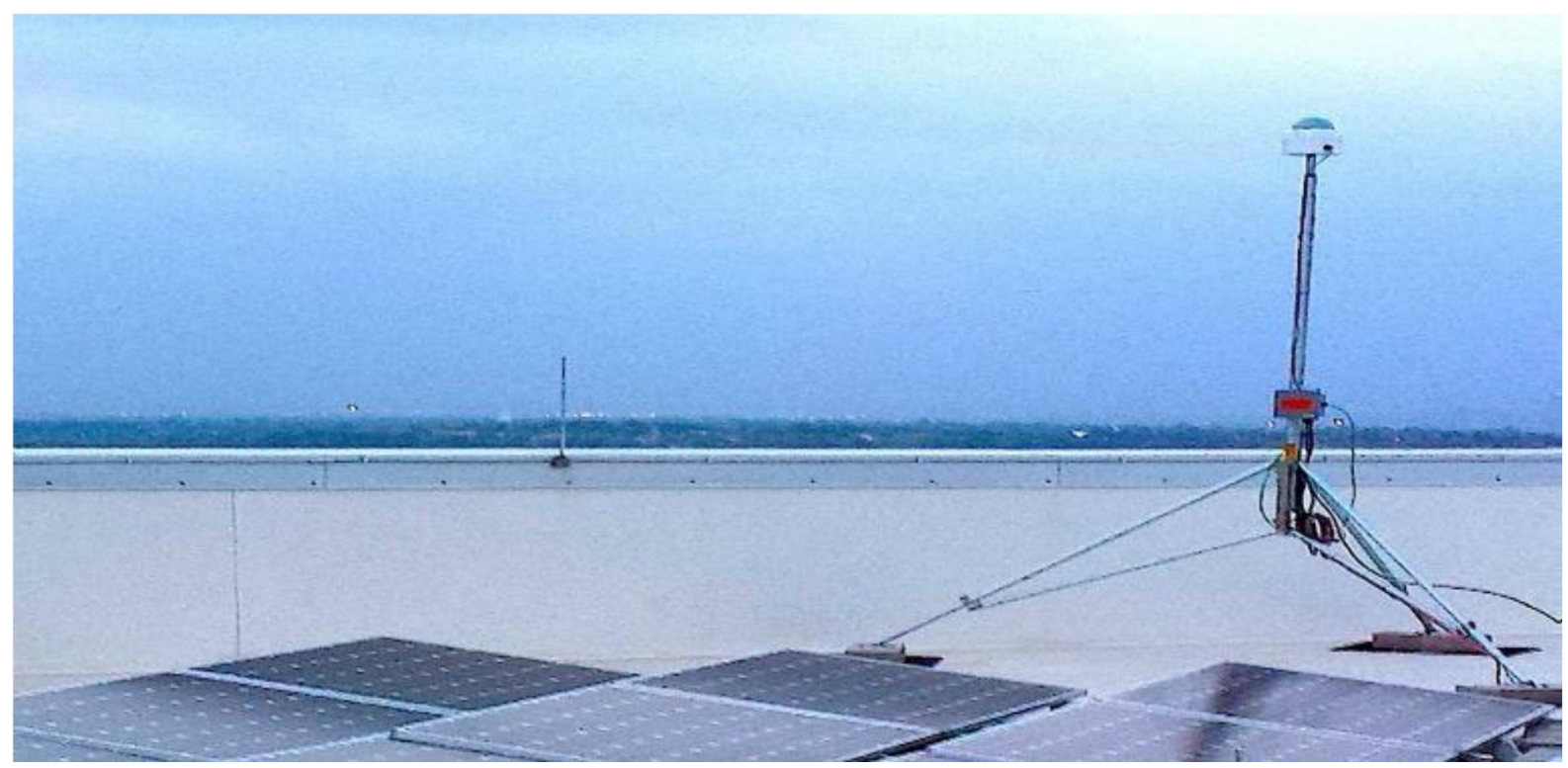

Figure 55 UTSA Sky Imager deployment at the NREL 


\section{Microgrid Management System}

\subsection{System Overview}

The Siemens Spectrum Power 7 Microgrid Management System (MGMS) is a product (pre-existing technology) available under license from Siemens that was integrated with the OpenFMB as part of this project. It is a software solution for optimal microgrid management and control that is based on the Siemens Spectrum Power 7 SCADA/EMS platform. The Siemens MGMS includes advanced functions such as seven-day load and generation forecasting, resource optimization, load shed, seamless planned transition to and from island mode, and market participation applications. The MGMS platform can be applied to all microgrids, whether they are grid connected or islanded, and whether the microgrid is a participant in an electricity market or not. MGMS can be deployed with implementation of a new microgrid or as an enhancement in an existing microgrid.

\subsection{Microgrid Architecture}

A microgrid is a set of co-located loads, and generation resources which may be connected to a utility grid through one or more points of common coupling as shown in Figure 56. In the context of this project, the generic microgrid includes generation from renewable resource, loads, and energy storage, and may be disconnected form the utility grid for islanded operations. This ability to operate either connected to the grid or when separated from the grid as an island, provides the critical loads within the microgrid with highly reliable power.

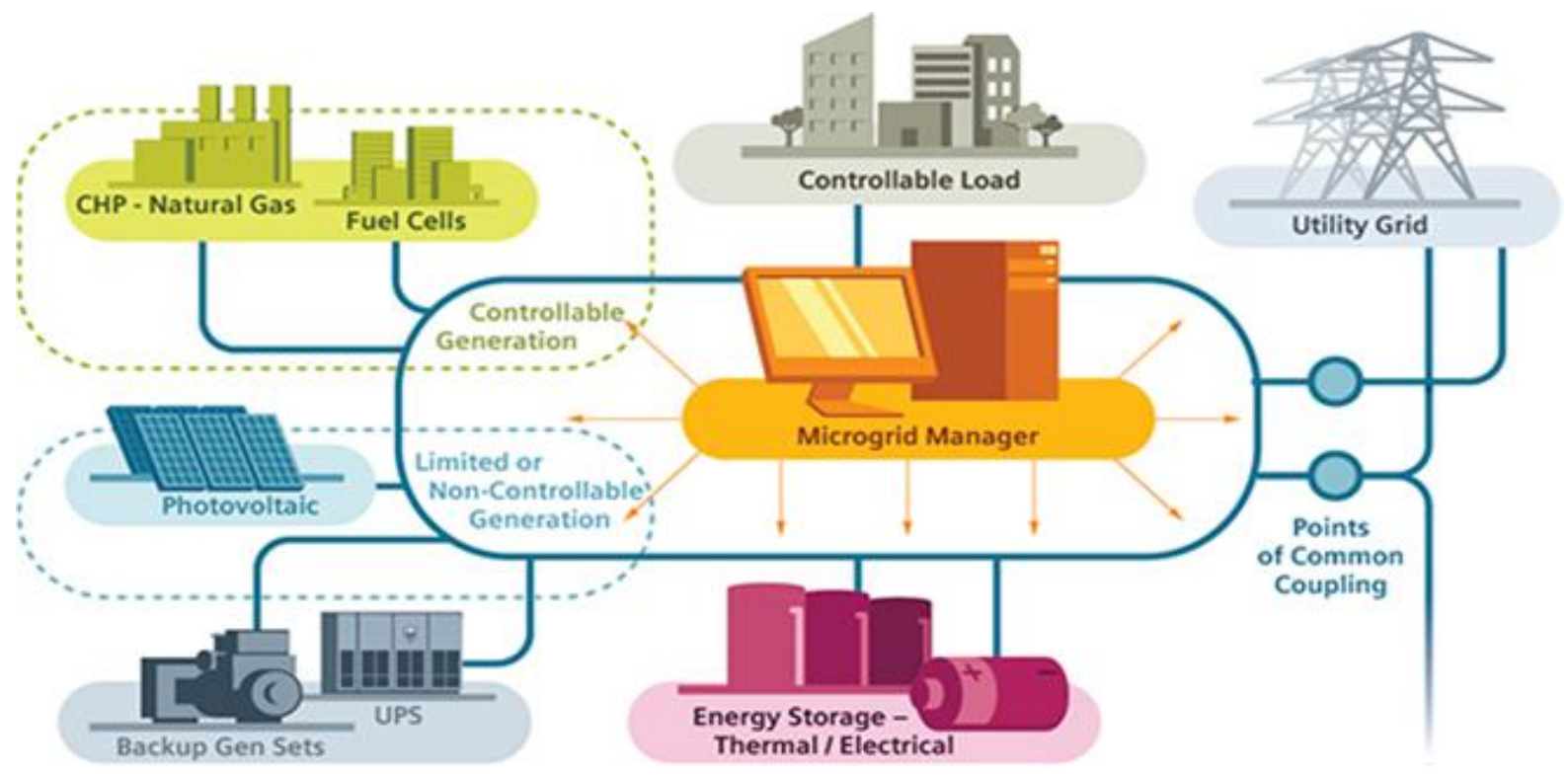

Figure 56 Generic Microgrid 
An MGMS provides for the management of the microgrid in terms of monitoring, control and optimization of the use of resources within the microgrid. The Architecture of the Siemens MGMS, shown in Figure 57, consists of several domains that manage the primary power system equipment and renewable energy components. The Primary Control Domain consists of controllers that operate in real time such as relays, fast voltage control, etc. The Secondary Control domain includes control that occurs on the order of a few seconds such as SCADA monitoring and control, frequency regulation, voltage setpoint control, and archiving. The tertiary control includes control that occurs on the order of minutes such as optimization, forecasting, scheduling and dispatch.

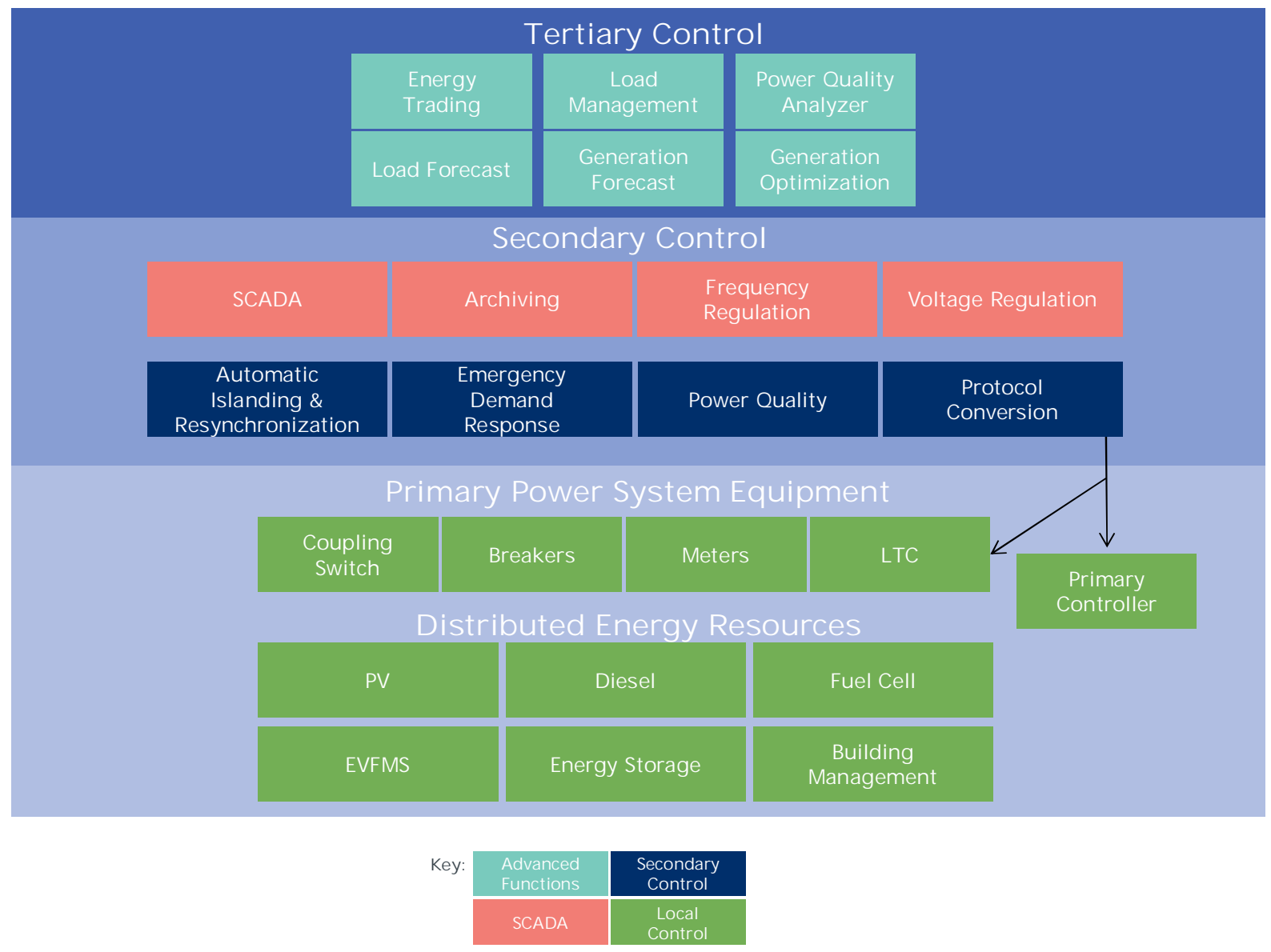

Figure 57: MGMS Architecture

\subsection{Siemens MGMS Functionality}

The functionality of the Siemens MGMS is selectable through configuration. For this project the following functionality was configured for use in the NREL ESIF and utility demonstrations. 


\subsubsection{SCADA}

The Siemens MGMS provides complete SCADA functionality for secure, reliable and efficient operation. The user interface provides a clear and easy-to-operate user environment including:

- A common look and feel for control centers, offices, remote, and mobile workplaces using web-based technology.

- Webstart technology that assures minimum start-up times of the user interface and provides zero installation clients

- Intuitive operation based on windows, menus, tooltips, toolbars, context menus, drag \& drop, context-sensitive help

- Powerful search, filter and sorting functions in all lists

- Easy data export to spreadsheets for reporting and further evaluation

- Task-oriented views including advanced visualization, for example, contouring.

\subsubsection{Generation and Load Management (GLM)}

The GLM module regulates the real power output of the generating units within the microgrid to maintain the desired frequency when in island mode and to maintain net interchange with the external grid when in grid connected mode. GLM uses an optimization function to satisfy system economic and regulating requirements in the most economic manner consistent with the operating capabilities of the microgrid.

\subsubsection{Generation Forecast and Optimization}

The MGMS advanced optimization model allows each microgrid owner to determine at an aggregate level whether to optimize generation dispatch based on economics or emissions or a combination of the two. Interfaces to a variety of weather forecasting services are supported to forecast renewable generation production while other interfaces to local building automation, metering systems, or SCADA systems provide the data for on-site generation needs. Based on demand forecasts, MGMS will optimize the mix of energy produced between all forms of generation, both electrical and thermal as well as the optimal use of battery energy storage systems within the microgrid. MGMS provides day-ahead and week-ahead generation schedules for resources such as solar, wind, storage, diesel, combined heat and power (thermal) that are the results of advanced economic and emission optimization.

\subsubsection{Load Forecast}

MGMS utilizes historical load data as well as seasonal weather conditions to forecast load profiles within the microgrid over hourly and weekly intervals. This forecast is then used to optimize the mix of energy generation, which results in the generation forecast. MGMS collects load data from a variety of sources including building automation systems, sub-meters and more to obtain a detailed view of the entire microgrid system. When loads are adjusted, MGMS makes automatic corrections to ensure forecast stability. 


\subsubsection{Load Shed}

MGMS performs the shedding or disconnecting of loads when requested by an operator or automatically during disturbance conditions to assist the utility to maintain system stability. The function has a modular design that can be adapted to meet specific requirements. The loads are virtually ordered according to predetermined priority schemes. Thus the sequence of events can be controlled and the most important loads remain connected.

\subsubsection{Archiving}

The Spectrum Power Historical Information System (HIS) provides a solid and reliable archive to store power system historical data. Features include periodic and spontaneous data collection, integrated data reduction and compression, a calculation engine, manual update capability, disturbance data collection, replay, easy integration with external tools, and an energy accounting module.

\subsubsection{MGMS Integration Points}

The Siemens MGMS integrates seamlessly with existing IT systems applications and power system assets. The Siemens Service-Oriented Architecture (SOA) Adapter Framework (SAF) provides web services-based APIs and configurable patterns which can be used to transmit and receive data to other applications using an Enterprise Service Bus or JBoss interface. Standard telecontrol communications capabilities include IEC 60870-6 TASE2 (ICCP), IEC 60870-5-101, IEC 60870-5-104, MODBUS, and DNP3.

\subsection{Integration of the Siemens MGMS with the OpenFMB architecture}

The Siemens MGMS was integrated with the OpenFMB Architecture using several different integration points described in Section 6.3.7.

- DNP3/DDS Adapter

- DDS/Web Services Adapter

- DDS/COP/Oracle

\subsubsection{DNP3/DDS Adapter}

In this integration approach, the MGMS, acquires data and issues control using the DNP3 protocol. These scans and commands were translated in a DNP3/DDS adapter to achieve integration and interoperability with other components in the OpenFMB Architecture. This approach was used as part of the Duke Energy and CPS Energy demonstrations (Section 8, 9)

\subsubsection{DDS/Web Services}

In this integration approach the MGMS transmits and receives data using its web services API. An adapter from web services to DDS was created to achieve integration and interoperability with other components that are part of the OpenFMB Architecture. 


\subsubsection{DDS/COP/Oracle}

The MGMS maintains schedules for load and generation forecasts in an application called the Current

Operating Plan (COP), which is based on the Oracle RDBMS. The results of the optimization processes can also be accessed from the COP through a Siemens API based on Oracle. As part of this project an adapter was created that allows these schedules to be transmitted/read to the OpenFMB Global Data Space. This adapter was used to integrate the UTSA forecasting applications with the MGMS optimal scheduling and dispatch functionality. 


\section{Validation at NREL ESIF}

\subsection{Objectives of Test}

This chapter describes the validation of the project concept at the NREL ESIF lab in Golden, Colorado. The objective of the testing and validation at NREL included the following major topics

- Validate OpenFMB

- Validate Siemens MGMS ability to control a generic microgrid

- Validate UTSA's STLF

\subsubsection{Validation of OpenFMB}

The objective of this set of tests at NREL was to validate the concepts of the OpenFMB architecture. Particular attention was placed on validating the ability of the OpenFMB to support a Monitoring and Control System on a distributed platform that can be hosted at the edge of a distribution grid. Particular attention was also focused on validating the ability of the OpenFMB to allow disparate intelligent devices with different native protocols to interoperate using adapters to the OpenFMB. The ability of the OpenFMB to host different transport protocols at the middleware level was also an objective of the test.

\subsubsection{Validation of the Siemens MGMS ability to control a generic microgrid}

The objective of this set of tests was to validate that the Siemens MGMS can be integrated with the OpenFMB and used to monitor and control a microgrid. It was also a test objective to validate whether the MGMS can successfully optimize the resources of a microgrid to give a day ahead schedule and to adaptively update that schedule during the operating day. The ability of the MGMS to provide anticipatory schedules and mandatory dispatch instructions to the controllable resources of the microgrid was validated.

\subsubsection{Validation of short term forecasting}

The objective of this set of tests was to validate the concept of acquiring forecast input data locally and forecasting load and solar PV generation locally. The ability of the OpenFMB architecture to host forecasting applications on a distributed platform was also tested. The accuracy of the forecast applications was also measured with various standard error statics.

\subsection{Technical Approach to Validation at NREL}

The project concept calls for a hierarchical control system made up a set of microgrids that are controlled by individual control systems that collaborate with higher level utility or regional control systems as an approach to allowing the grid to support higher levels of contributions from renewable resources. The 
scope of the project called for the use of the NREL ESIF to set up a generic microgrid using NREL simulators and to apply the control concept to the simulated microgrid.

After successfully demonstrating control of a simulated microgrid, it can be shown by induction, that it is possible to control two or more microgrids that are part of the hierarchy and that the proposed control scheme should work. The technical approach consists of focusing on a simulated microgrid in each of the following major project steps:

- Specification of the simulated microgrid

- Development of the Architecture of the control system

- Design of the component interfaces

- Configuration of the MGMS

- Development of a Frame Test Specification

- Development of Test Books

- Configuration of the Adapters

- Set up of the Microgrid

- Execution of the tests and recording of results

- Demonstration to industry

\subsubsection{Specification of the simulated microgrid}

This step consisted of specifying a microgrid using the simulators at the NREL ESIF. The simulated microgrid contained critical and non-critical loads, generation from simulated wind power resources, generation from simulated solar power resources, a battery energy storage systems and a diesel generator. The microgrid was documented in [4].

\subsubsection{Development of the Control System Architecture}

This step included the development of the architecture of the system that controls the microgrid. The functional and non-functional requirements for the control system, the architectural drivers, the basic principles, the basic decisions, a high level system decomposition, and interfaces between various components in the microgrid were documented in [1], [2]. The relationships of the OpenFMB Architecture to other widely recognized industry accepted architectures, such as the Gridwise Architecture Council's Interoperability Context Setting Diagram (the GWAC stack) and the Smart Grid Architectural Model (SGAM), were developed and the key concepts of the OpenFMB Architecture were applied to the control scheme.

\subsubsection{Component Interfaces Information Exchange Modeling}

This step consisted of designing the information exchanges among the various components of the microgrid system. The methods and processes suitable for determining the structure of the message payloads were documented in [2]. 


\subsubsection{Configuration of the MGMS}

This step consisted of configuration of the Siemens Spectrum 7 MGMS and population of the MGMS primitive data management system models with the data that describes both the power system characteristics of the simulated microgrid and the communications and control system used to manage the behavior of the microgrid [3].

\subsubsection{Development of the Frame Test Specification}

This step developed the Frame Test Specification as the basis for the testing of the key elements of Open Field Message Bus Architecture at the NREL ESIF laboratory. It provided for the demonstration and proof of concept of the overall approach as well as the testing of the products and standards chosen. It contains all super ordinate process steps that focus on the quality of the solution. [4]

\subsubsection{Development of Test Books}

Test books were written that documented test procedures with sections for each component or subsystem that was under test. In each section, the pre-requisites for the test section were recorded. Required test cases were described, and the tests were documented in a step by step manner. Each step has a description of the action to be taken by the tester, and of the expected results following execution of the test step.

Two test books were written [5], and [6]. The first test book documented the testing of the MGMS controller, and the second test book treated the testing of the Forecasting Applications. In the case of the Siemens MGMS, the MGMS itself was tested as part of the formal release of the MGMS as a Siemens product. This meant that testing at NREL could be focused on testing on the specific areas of interest to the project: integration of the MGMS with the OpenFMB, and the ability of the MGMS to produce Day Ahead and Intra-day optimal schedules. The test book for the Forecasting Applications, focused on testing the integration of the applications with the OpenFMB, and on the accuracy of the application.

\subsubsection{Configuration of the Adapters}

This step consisted of configuration of the adapters with the maps that were developed as part of [5]. Configuration included editing XML to depict the relationship been the addresses of the target protocol and the topic and attributes of the Global Data Space that exists on the distributed OpenFMB filed bus.

\subsubsection{Setup of the Microgrid}

The next steps are described in summary format in this section to maintain the logical flow of the steps of the project. Additional information is provided in later sections.

This step consisted of setting up the microgrid at NREL ESIF using the simulators for various renewable resources. The setup was done with the assistance of NREL staff engineers

\subsubsection{Execution of the test}

The next steps are described in summary format in this section to maintain the logical flow of the steps of the project. Additional information is provided in later sections. 
The validation tests were conducted on site at ESIF by OMNETRIC and NREL staff working as a team. The test results were recorded in a test report. [7]

\subsubsection{Demonstration to the Industry}

Following completion of the formal test, a demonstration was held for the NREL staff and for stakeholders from the industry. This demonstration was conducted on May 24, 2016.

\subsection{Configuration of NREL Equipment into a Simulated Microgrid}

The facilities of the NREL Energy Systems Integration Facility in Golden, CO were used to configure a simulated generic MW scale microgrid as shown in Figure 58, Simulated Microgrid. The simulated microgrid included a variety of different types of resources including renewable and conventional resources: generation from wind power, generation from solar PV arrays, a battery energy storage system, as well as controllable loads and critical loads.

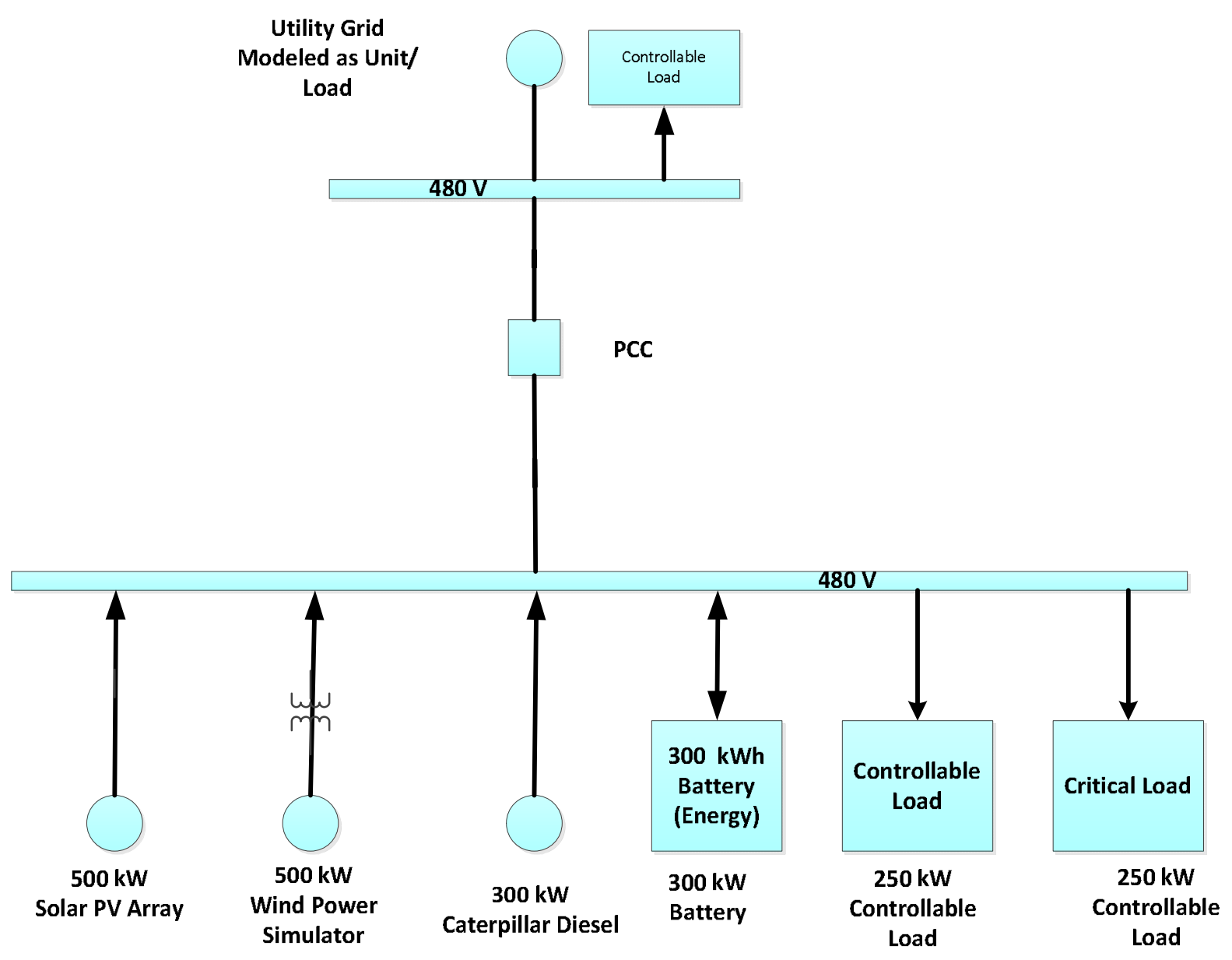

Figure 58: Simulated Microgrid 
The simulated microgrid used the following facilities of the laboratory:

\subsubsection{Research B Bus, 3 phase 480 VAC}

The ESIF Research Electrical Distribution Bus (REDB) was used to connect the resources of the microgrid. This bus was operated at $480 \mathrm{Vac}$, with a five wire design: A, B, C phases plus neutral and ground wires at $60 \mathrm{~Hz}$. The bus has the capacity to operate at 600 amperes

\subsubsection{The Erigo Eagle Picher $300 \mathrm{~kW}$ Battery Energy Storage System}

The Battery Energy Storage System used in the microgrid was a $300 \mathrm{~kW} / 410 \mathrm{kWh}$ battery from Erigo Eagle-Picher. The BESS includes three tiers with different technologies:

- Lead Acid (PbA)

- Lithium Ion Iron Phosphate (LPF)

- $\quad$ Nickel Cadmium $(\mathrm{NiCd})$

The battery includes built-in bi-directional inverters. One ABB PCS 100 inverter supports the NiCd tier, a second ABB PCS 100 inverter supports the LFP tier, and a Princeton GTIB 480-100 MXFD inverter supports the $\mathrm{PbA}$ tier.

\subsubsection{Load Banks}

The microgrid loads were represented by two R-L-C load banks supplied to the NREL ESIF by LoadTec that can be controlled by software over a range of 0 to $390 \mathrm{~kW}$ in steps of $50 \mathrm{VA}$ per phase. One of the load banks was configured as a critical load while the other was configured as shedable load. Both loads were driven by software scripts during the demonstration. Load data from the NREL RSF 2 building was recorded at 15-second intervals and used as input to the scripts driving the load banks in the microgrid. This control script reproduced the variable behavior of a building at scale in the microgrid. The control script allowed this behavior to recreated (started/stopped/etc.) on demand during the testing of the project concepts.

\subsubsection{Diesel Generator}

The microgrid generation includes a variety of types of generation. The conventional generation was represented by a $300 \mathrm{~kW} 3$-phase Caterpillar XQ30 diesel generator. The conventional unit EMCP4 RTU interface was used for remote monitoring and control of the unit.

\subsubsection{Wind Power Generator}

Wind power generation for the microgrid was simulated at scale with the ESIF's AEC 2660 DC supply and a Schneider XC 540 Inverter. The project recorded the output of NREL wind turbines at 15-second interval over time horizon of several days and used this data to create control inputs to the DC supply, which was then used as input to the Schneider XC540 inverter. Using this approach the output of the XC 
540 inverter reproduced the variable behavior of a typical wind farm at scale in the simulated microgrid. The control script allowed this behavior to be recreated (started/stopped/etc.) on demand during the testing of the project concepts.

\subsubsection{Solar Power Generation}

A similar approach was used to simulation of Solar PV generation in the microgrid. The output of the Solar PV system at the NREL RSF 2(Research Facility building two), was recorded in 15-second intervals over a three day period. This data was used to create control inputs to the ESIF Magna Power MTD 1000 PV Simulator which was then used as input to the ESIF AE 500TX Inverter. The AE 500TX inverter was connected to the REB B bus at $480 \mathrm{VAC}$.

Figure 59 illustrates the load and renewable generation patterns that were recorded and used to develop the control scripts in the simulated microgrid.

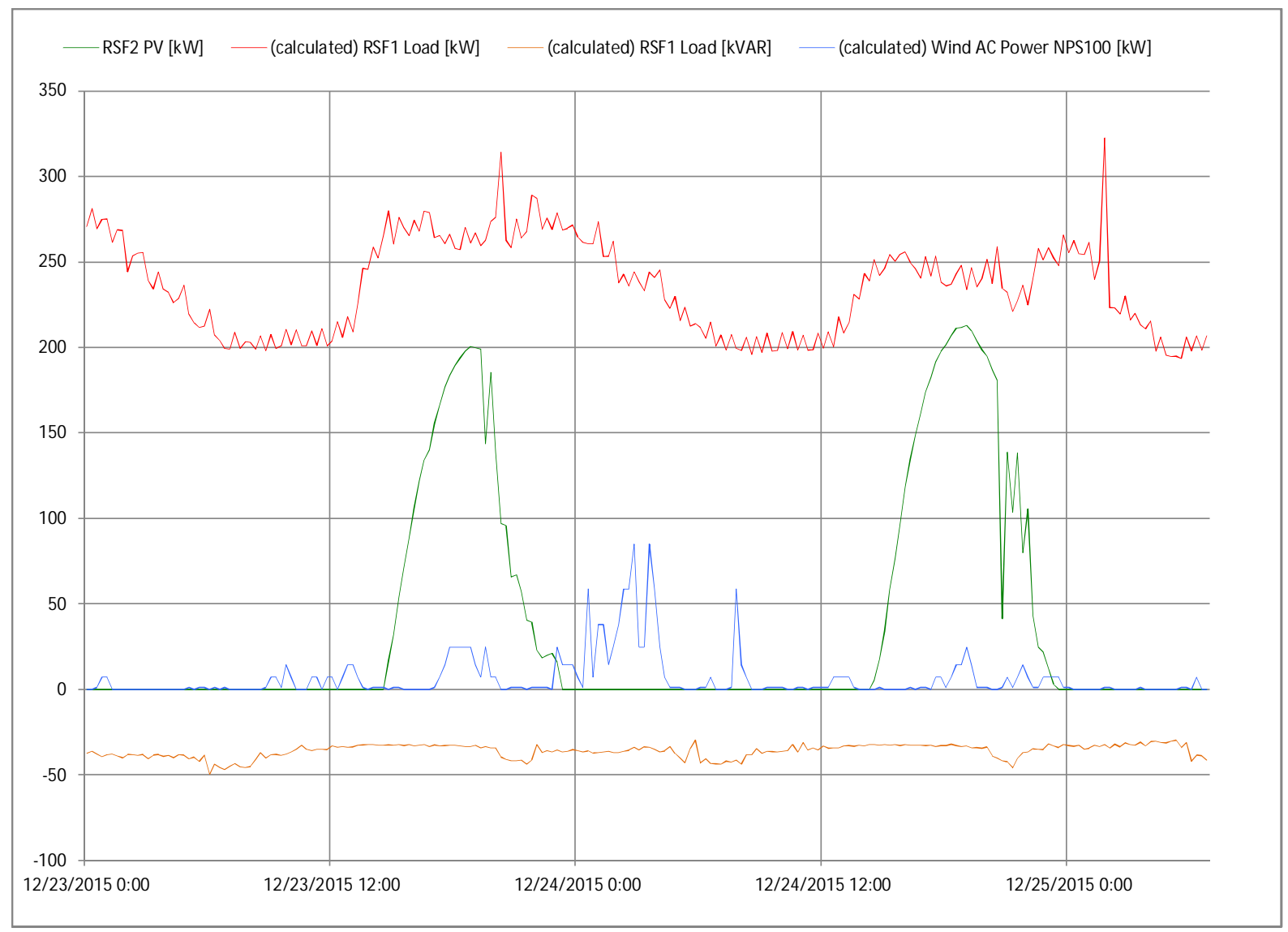

Figure 59 Microgrid Load and Generation Data. 


\subsubsection{Grid Simulator}

Microgrids are usually interconnected to a larger utility grid at a point of common coupling (PCC). The simulated micro grid at NREL ESIF was configured so that it could either connect to the actual XCEL Energy utility grid through a protected switch or to a Grid Simulator, as shown in Figure 60. The Grid Simulator was supplied by Ametek was set up to operate as a constant voltage source of 480 Vac.

\subsubsection{Protection, Isolation, and Bonding}

Protection, Isolation and grounding analyses were done on the simulated microgrid by the project team and by NREL staff. The results were included in the microgrid with relays, fuses, and switchgear as shown in Figure 63.

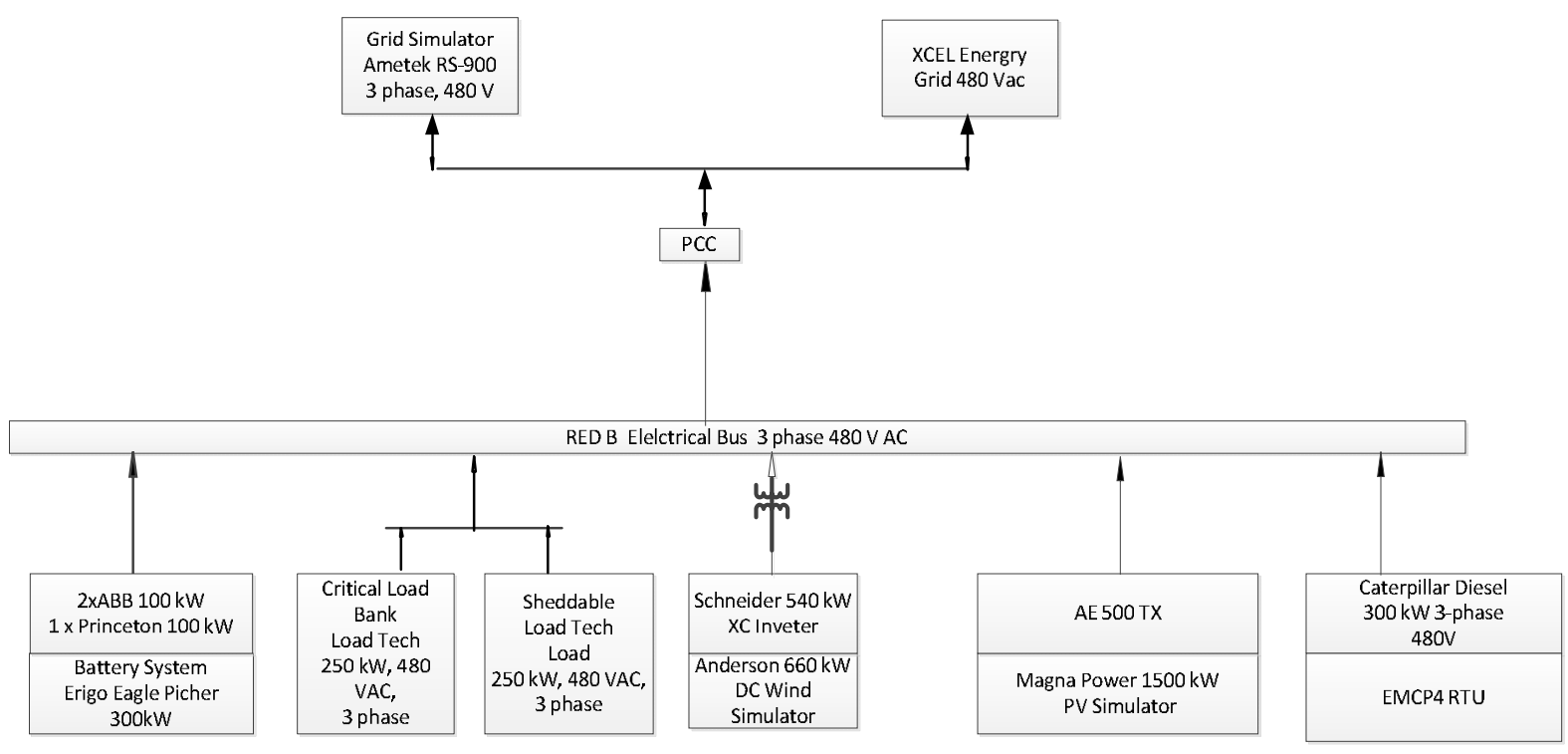

Figure 60: Simulated Microgrid at ESIF

\subsection{Monitoring and Control System at NREL}

A system was set up to monitor and control the simulated microgrid at NREL ESIF described in Section 7.3. The system was hosted as shown in the deployment diagram in Figure 61. The following are the major components of the deployment. 


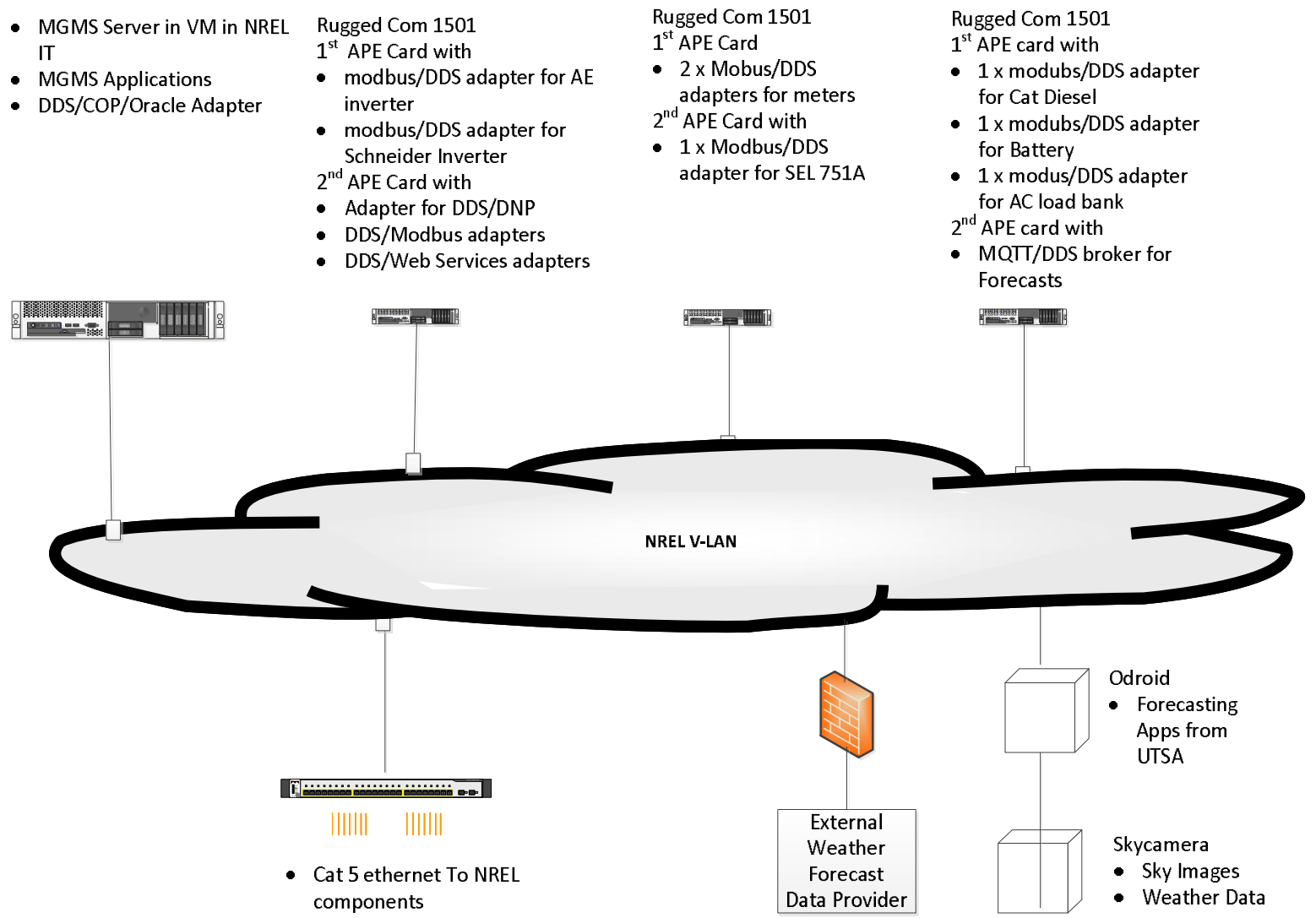

Figure 61: Deployment Diagram

\subsubsection{Communications Nodes}

Two Siemens RuggedCom 1501 Utility Grade Layer 2/3 Switch and Routers with Application Programming Environment (APE) cards were used as communications nodes to host the OpenFMB including: OpenFMB adapters, DDS, and the MQTT broker. These communications nodes support the Ubuntu operating system, and the Java programming environment. It was used to support applications, communications, and the OpenFMB Interface layer. The form factor selected for deployment was the 19inch rack mount subsystems, were located in the NREL ESIF PSIL control room.

The Hardkernel Odroid was used to host the forecasting application and to publish forecast results as schedules using MQTT protocol. This Odroid was located in the NREL ESIF PSIL control room

\subsubsection{MGMS Server}

The Siemens MGMS was deployed on a Hewlett Packard mobile server. The mobile server supported Spectrum 7 SCADA functionality, Optimal Scheduling and Dispatch, and a Historical Information System (HIS). The MGMS platform incudes the Redhat Linux operating system, the Oracle RDBMS, and IBM Cplex MIP solver. The server was packaged as a mobile workstation and was located in the NREL ESIF PSIL control room. 


\subsubsection{User Interface Clients}

User interface clients for the Siemens MGMS and for the Forecasting Applications hosted on the Odroid were provided. These UI clients were also located in the NREL ESIF PSIL control room. These clients ran on standard notebook computers that support the Internet Explorer or Firefox browser.

\subsubsection{Network Diagram at NREL ESIF}

The simulated microgrid communications and control system was set up on a virtual LAN at NREL as show in Figure 62. This VLAN was set up as a subnetwork in the ESIF to validate the OpenFMB concepts, and for testing of the MGMS and hierarchical control system concepts. The LAN used Ethernet over copper for combinations within the PSIL, and Wi-Fi (IEEE 802.X) for communications with the sky camera on the rooftop of the ESIF facility. The VLAN had controlled access through multiple firewalls to an external weather forecast data provider as described in section 5.

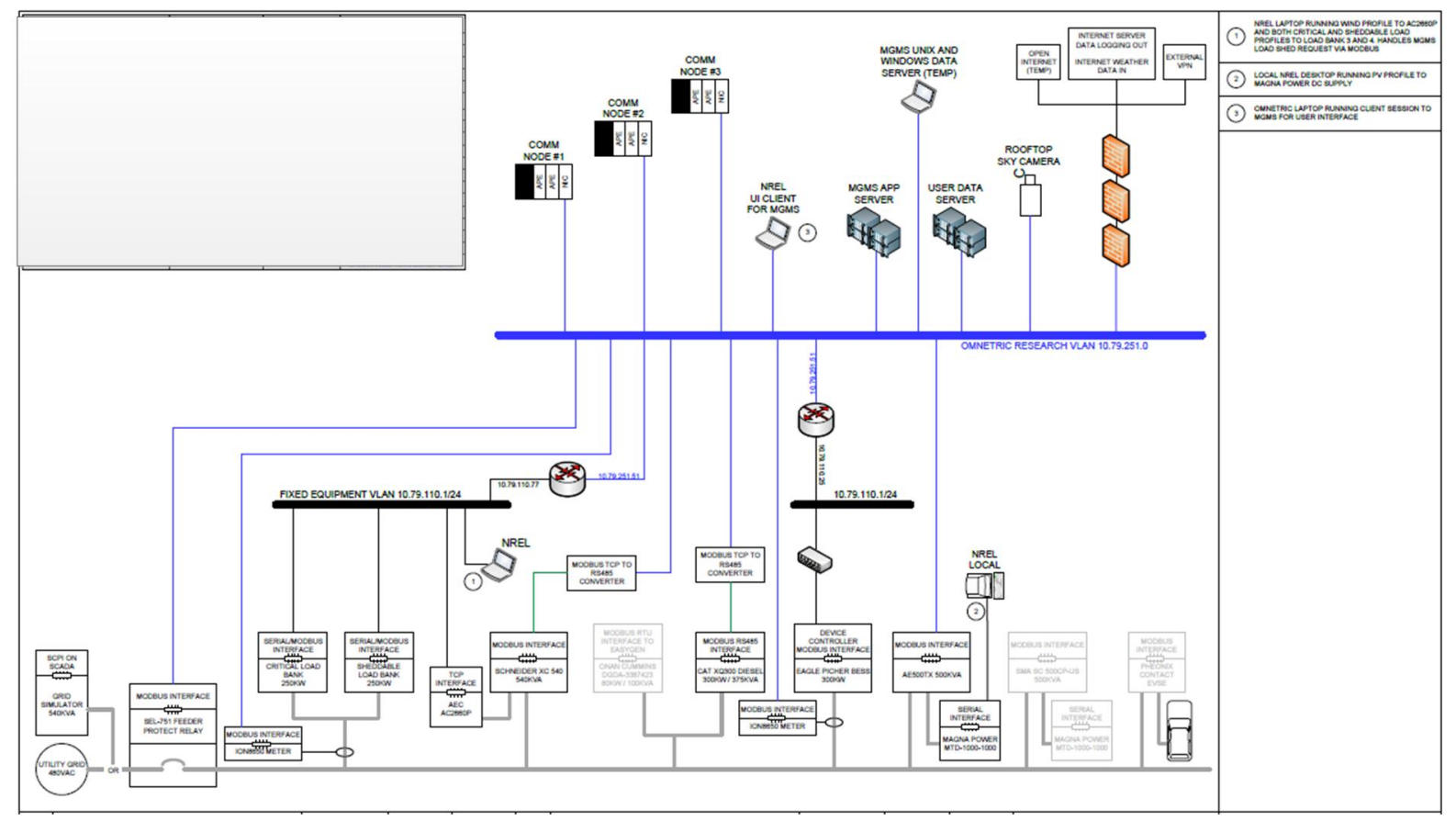

Figure 62: OMNETRIC Research VLAN

\subsubsection{OpenFMB Configuration}

This section describes the configuration of the OpenFMB. The Data Distribution Services licensable product from Real Time Innovations (RTI) configured with UDP/IP was selected for demonstration as DDS publish subscribe services. The Hive MQTT was also selected for demonstration as another publish subscribe service. 
OpenFMB adapters are also available from SGIP under open source licenses. These adapters were configured or enhanced to support the following:

- Modbus/DDS

- DDS/Modbus

- DDS/Web Services

- DDS/COP/Oracle

- DNP/DDS

The data model chosen for this implementation of the OpenFMB is based on the IEC CIM UML Model., Profiles of the CIM were developed by the SGIP for demonstration at Distributech 2016. These profiles define the global data space and are available in both IDL and XSD formats under the terms of the Apache License Version 2.0.

\subsubsection{Integrated Test Configuration}

The integrated test configuration is shown in Figure 24. This figure, prepared by NREL staff, gives an overview of the microgrid in the form of a power system one line diagram, with an overlay of the metering, communications, and control system interfaces. It also shows the protection of microgrid resources with relays, fuses, breakers and other associated switchgear.

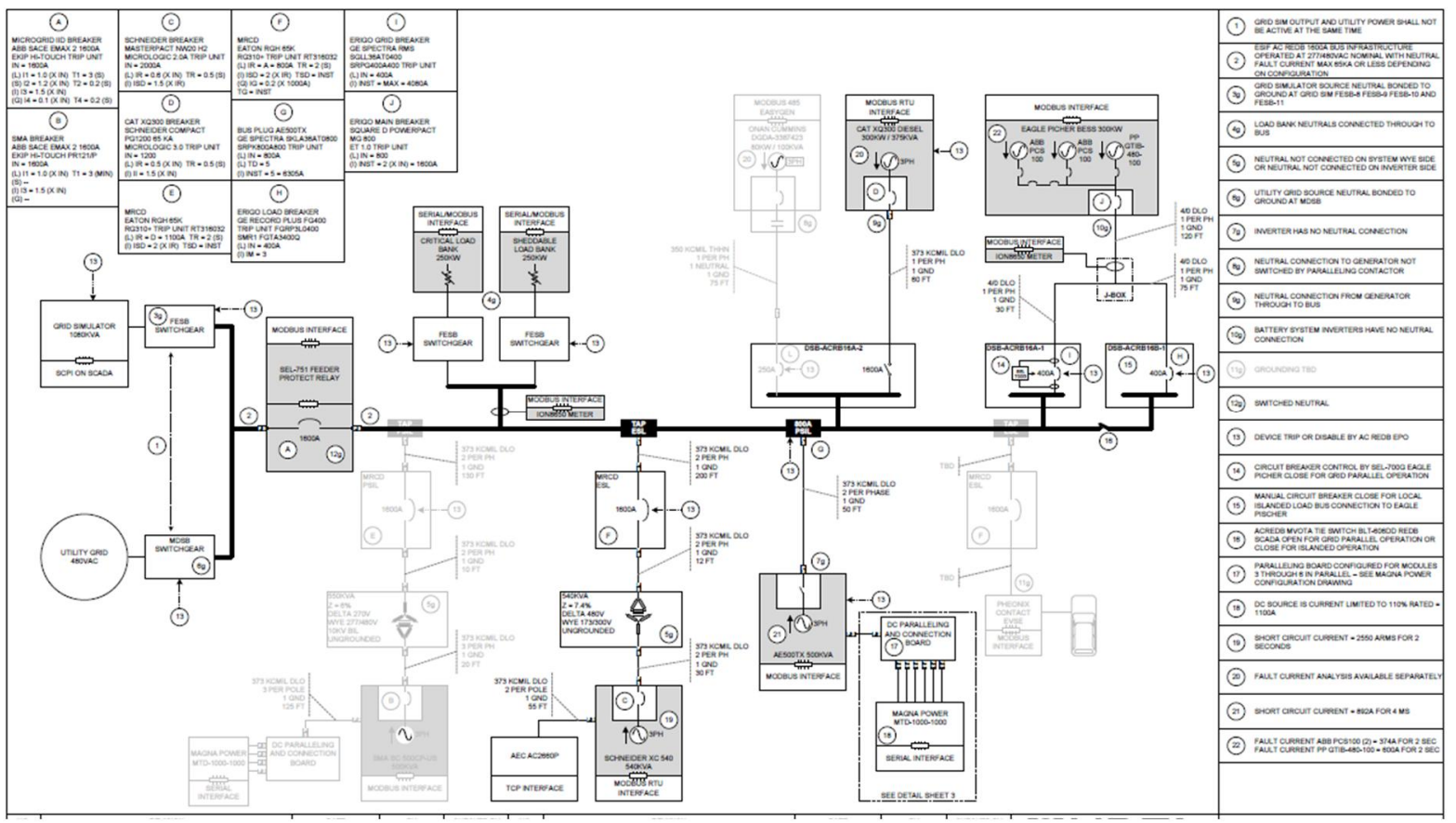

Figure 63 Microgrid Power System with Metering and Communications Interfaces 


\subsection{Testing and Test Results}

\subsubsection{Structured Testing}

Structured and unstructured tests were carried out at the NREL ESIF. Structured testing of the Forecasting Applications was carried using the test book [6]. Results are reported in Section 5 of this report. Testing of the OpenFMB was carried out using the test book [5], which thoroughly tested the publish/subscribe pattern for all communication paths shown in Figure 64. Testing included use of an implementation of the open standard DDS by RTI [8] for the transport layer of the OpenFMB Architecture. The use of the open source implementation of MQTT [9] as a transport layer of the OpenFMB was also verified.

Testing of the integration of the Siemens MGMS with the OpenFMB architecture was also included in the test book. This integration was also verified in the NREL ESIF, as a predecessor to actual demonstration at a utility site.

The results of the tests that validated the OpenFMB are recorded in [7].

\subsubsection{Unstructured Testing}

Additional unstructured testing was performed in the NREL ESIF lab. These tests included startup and shutdown sequences for some of the power system components, and part-task testing of communications to the controllers to support functionality that is like to become available in the near term. 


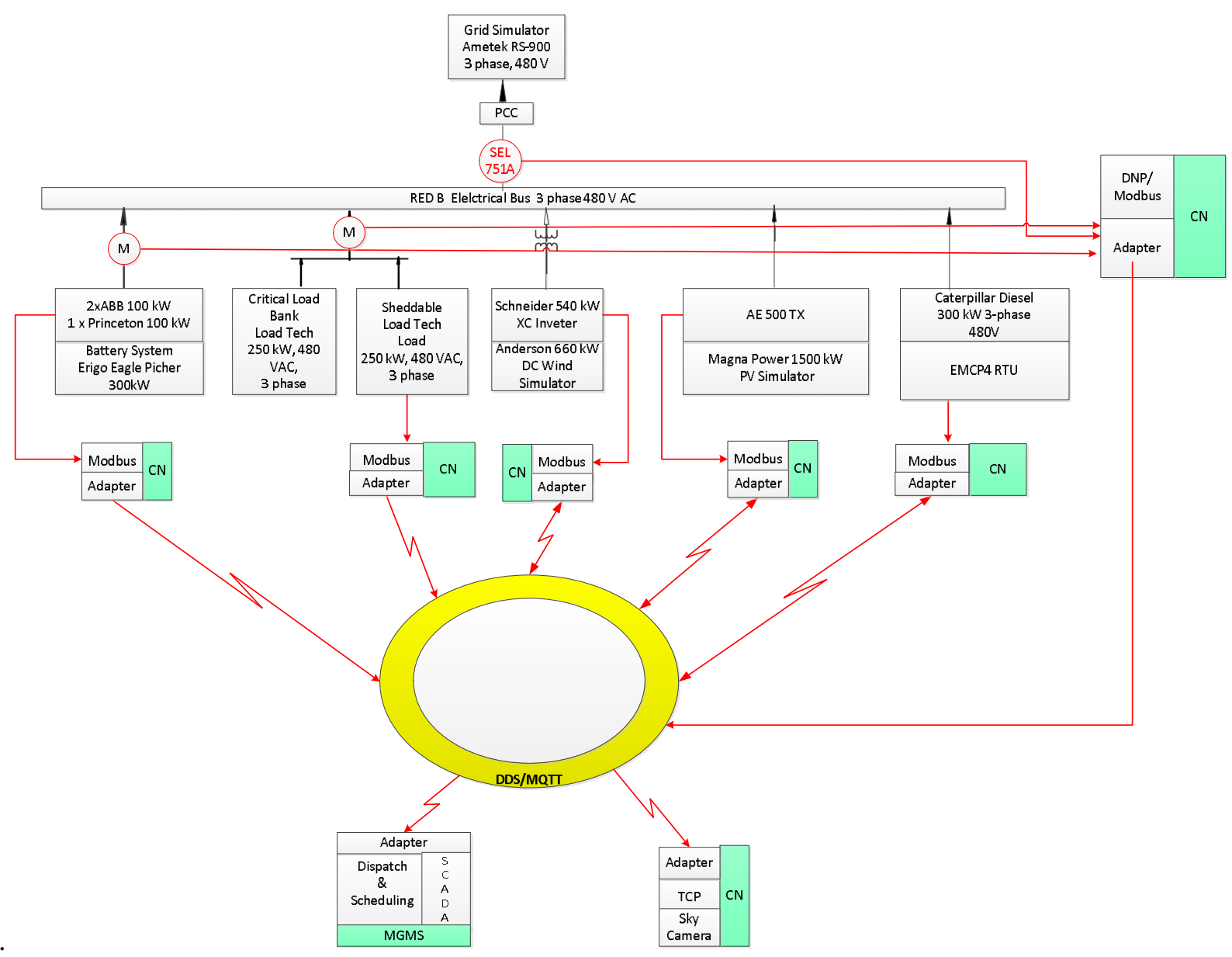

Figure 64: OpenFMB Communications Testing

\subsubsection{Demonstration}

A demonstration was given to NREL staff and to industry stakeholders on May 24, 2016. The demonstration included a review of the project concepts, the use of the ESIF components to setup a microgrid system at MW scale for testing of the OpenFMB, the MGMS, and the Forecasting Applications. The demonstration included optimization of the microgrid when connected to the utility grid, and monitoring and control of the microgrid when operating as an island.

A copy of the press release is included as Appendix A3.

\subsubsection{Discussion of results}

The testing at NREL's ESIF, in the opinion of the project team, verified the ability of an implementation of the OpenFMB Framework to successfully forecast load demand, and solar PV generation. It also demonstrated an MGMS integrated with the OpenFMB architecture that can optimally schedule the resources of a microgrid on both a day-ahead and an intra-day basis, and that can make these optimal schedules available to a hierarchical control system as envisioned in the project concept described in section 3. Real time optimal dispatch of the resources of the microgrid by the MGMS was tested and 
demonstrated. The testing verified the ability of the OpenFMB to support interoperability of multiple protocols, with the latencies required for monitoring, control, scheduling and dispatch of microgrid functions. 


\section{Field Demo at Duke}

\subsection{Duke Energy Microgrid at Mt Holly}

Duke Energy has set up a microgrid at its Training Center in Mount Holly, NC. The microgrid resources, loads, and switchgear components are shown in Figure 65 and in Figure 66 Mt Holly One Line Diagram. This section briefly describes the Mount Holly Microgrid (Mt Holly).

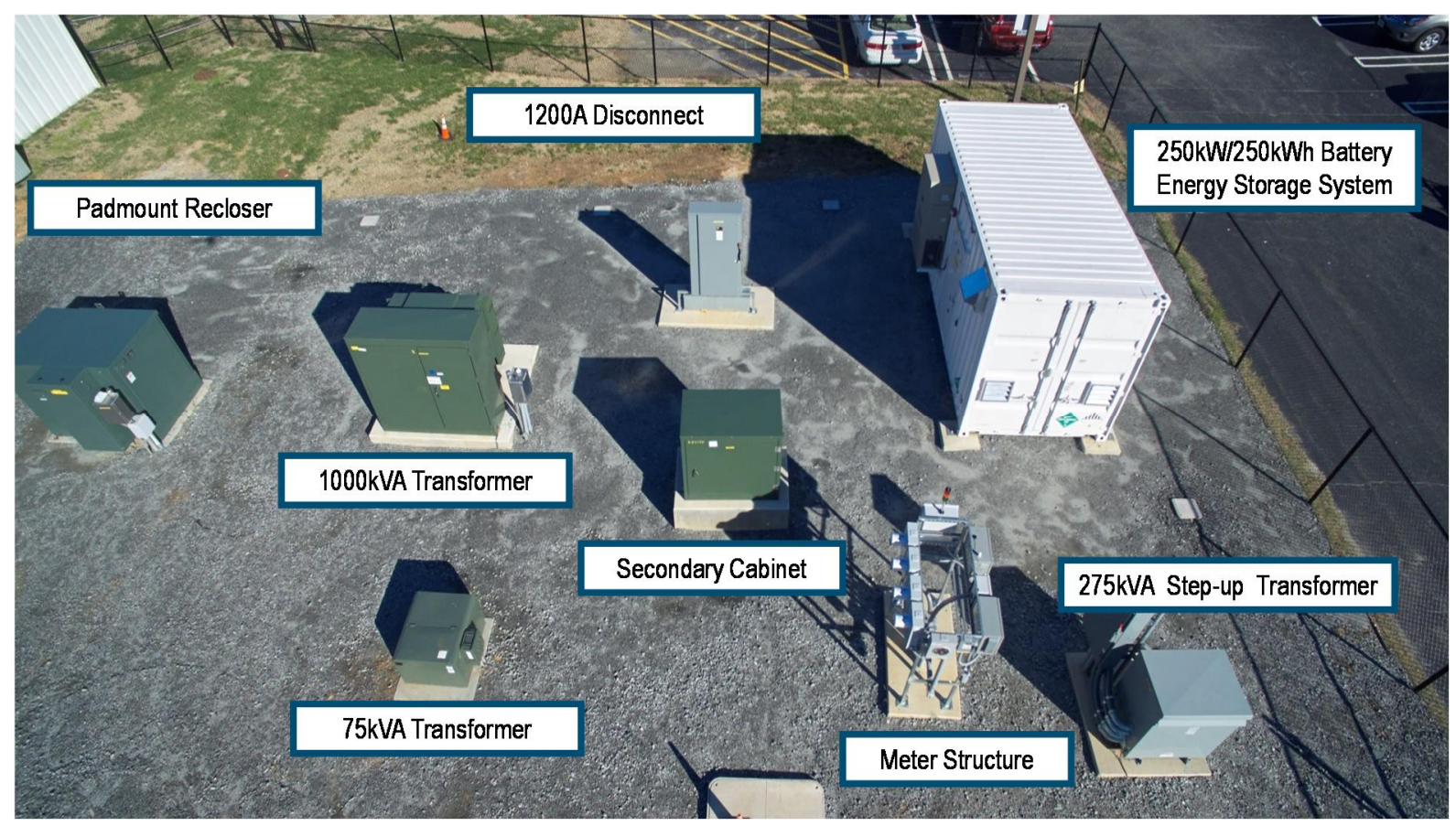

Figure 65: Mt Holly Microgrid Components 


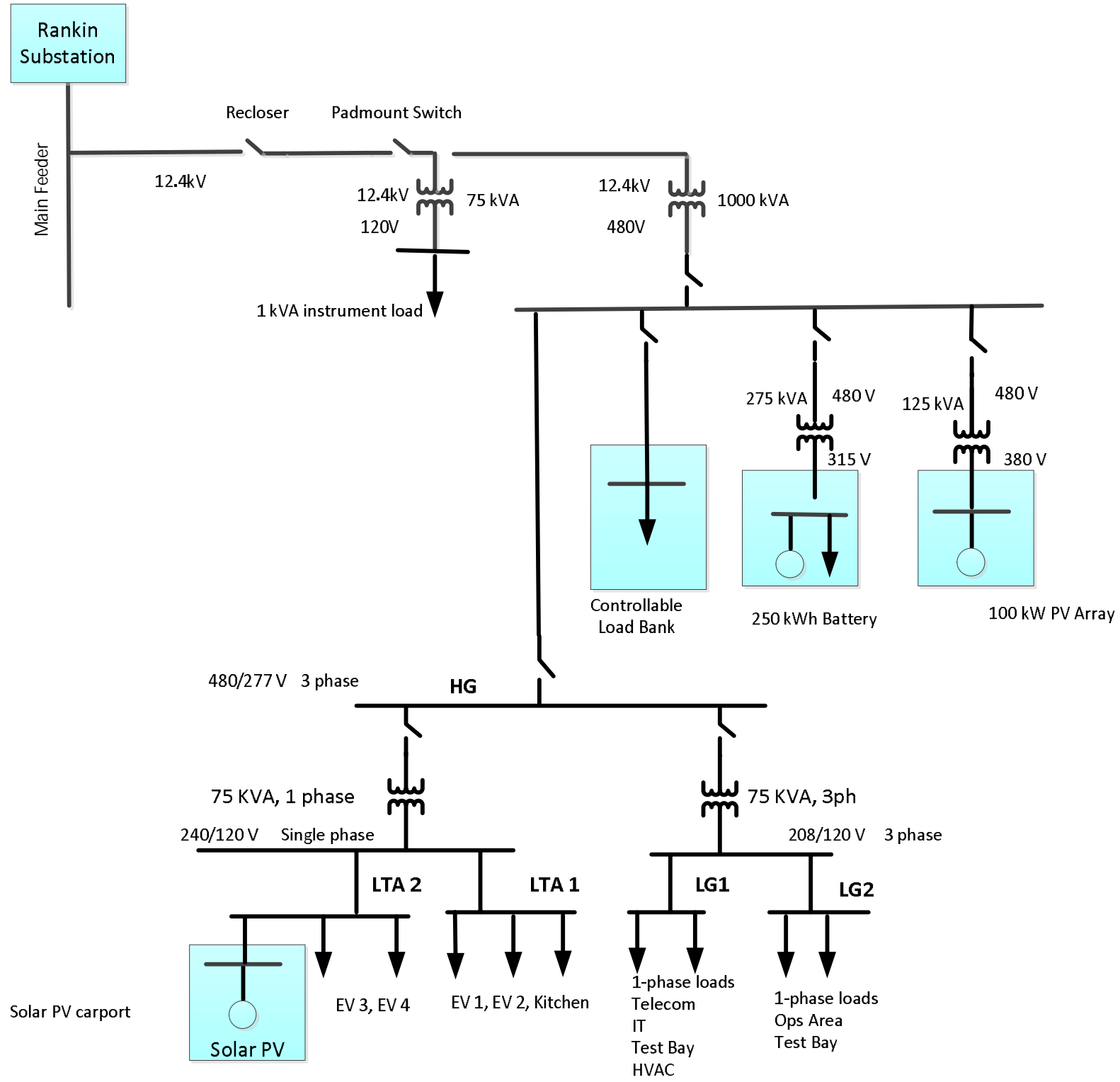

Figure 66 Mt Holly One Line Diagram

The Mt Holly facility includes a training center and laboratory facilities for the Duke Energy Emerging Technologies Office (ETO). A microgrid was established in the training center that includes the laboratory workshop facilities as the load of the microgrid. This load is variable within a typical range of 7 to $30 \mathrm{~kW}$, depending on the time of day and the facilities in use. These loads are connected to the 480 volt microgrid distribution system by two transformers: a $75 \mathrm{kVA} 3$-phase transformer, and a $75 \mathrm{kVA} 1$ phase transformer. The microgrid resources include a $100 \mathrm{~kW}$ solar PV array and associated inverters that are connected to the $480 \mathrm{~V}$ microgrid distribution system by means of a 380/480 volt transformer. A 250 $\mathrm{kWh}$ battery energy storage system (BESS) and a 500 controllable $\mathrm{kW}$ load bank make up the remaining microgrid resources. The Mt Holly microgrid operates under an agreement with Duke Energy that requires no reverse power flow from the microgrid into the utility feeder (feeder 1201) that originates in 
the Rankin substation. Duke has developed a real time control system that varies the consumption of the load bank and the charging/discharging of the BESS to ensure that there is no flow from the microgrid back into the utility feeder. Mt Holly also has a protection and control system provided by Duke which supports seamless, unplanned transition from grid connected to islanded operations, and planned reconnection from islanded to grid connected operations.

\subsection{Variability of Generation from Solar PV}

Duke has collected operating data from the microgrid for an extended period of time, covering a wide variety of weather conditions. The variability of generation from renewable resources is clearly shown for different weather conditions in Figures 67 and 68. Description of the point names on the graphic are referenced in Figure 70.

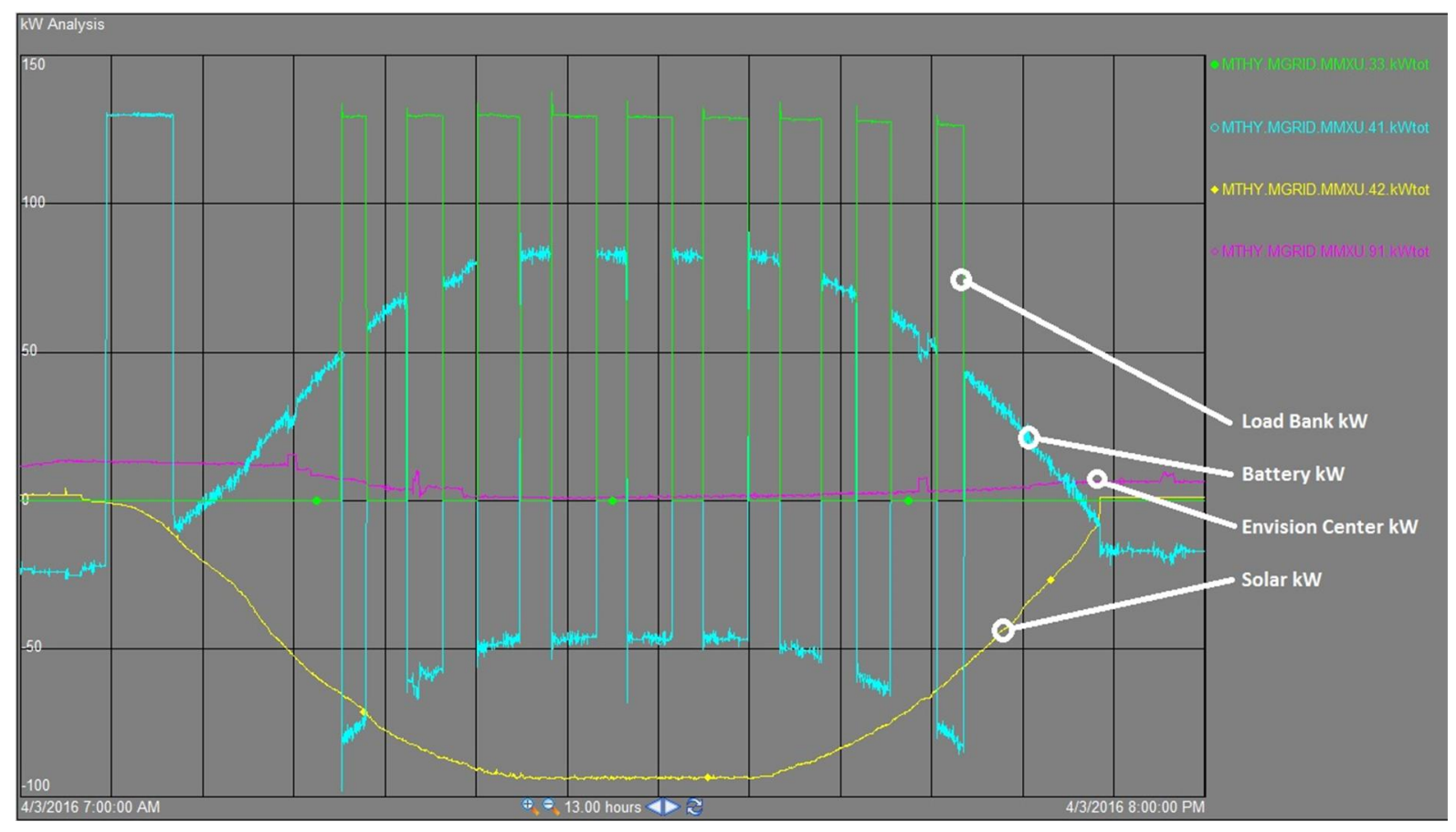

Figure 67: Solar PV Generation on a Clear Day 


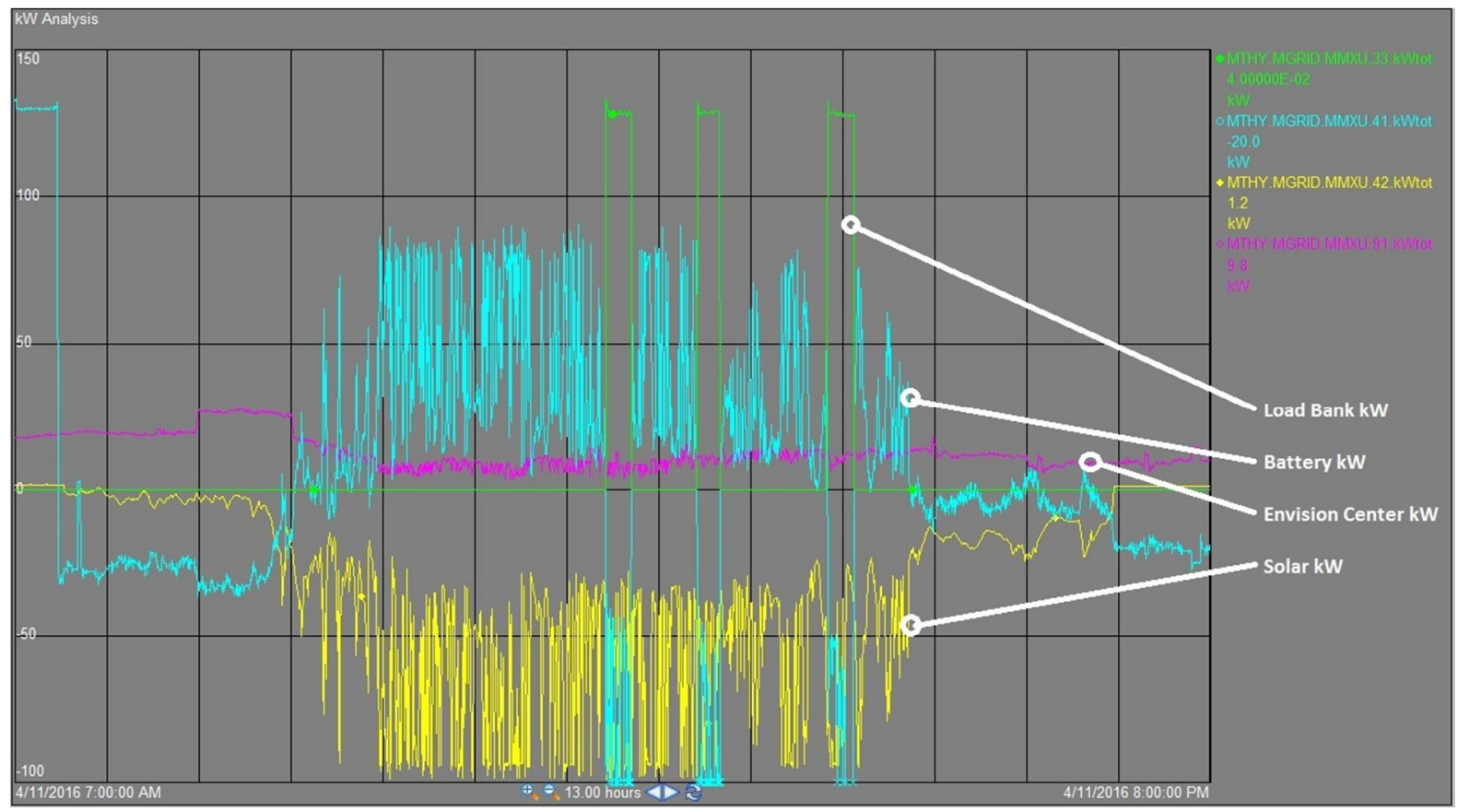

Figure 68 Solar PV Generation on a Cloudy Day with Rapidly Moving Sirius Clouds

\subsection{Monitoring and Control}

An implementation of the OpenFMB Framework and Reference Architecture [1] and [2] was set up by Duke Energy to monitor and control the resources of the Mt Holly Microgrid as shown in Figure 69. The Siemens MGMS was deployed on a virtual machine hosted on a CISCO Server supplied by Duke. The Duke real time control application was deployed on an industrial PC from Moxa. Data was recorded by a weather instrument station supplied by WeatherHawk, and forecast weather data was obtained from the weather underground web service [5]. Real time measurements were collected from Schneider PowerLogic ION meters using the DNP 3 protocol. This data was then converted to DDS by a DNP3/DDS adapter and published to the Global Data Space. Data from the Battery Management Systems (BMS) of the BESS, $100 \mathrm{~kW}$ PV inverter, and from the load bank controller were collected using the Modbus protocol. This data is then converted to DDS and published to the OpenFMB Global Data Space by Modbus /DDS adapters. The adapters were co-developed for the SGIP and the Coalition of the Willing II demonstrations and are available under the terms of an open source license from Apache. Hard wired media was used for physical connectivity of the LAN used by the OpenFMB. The PCC was connected to the OpenFMB LAN using 4GLTE. 


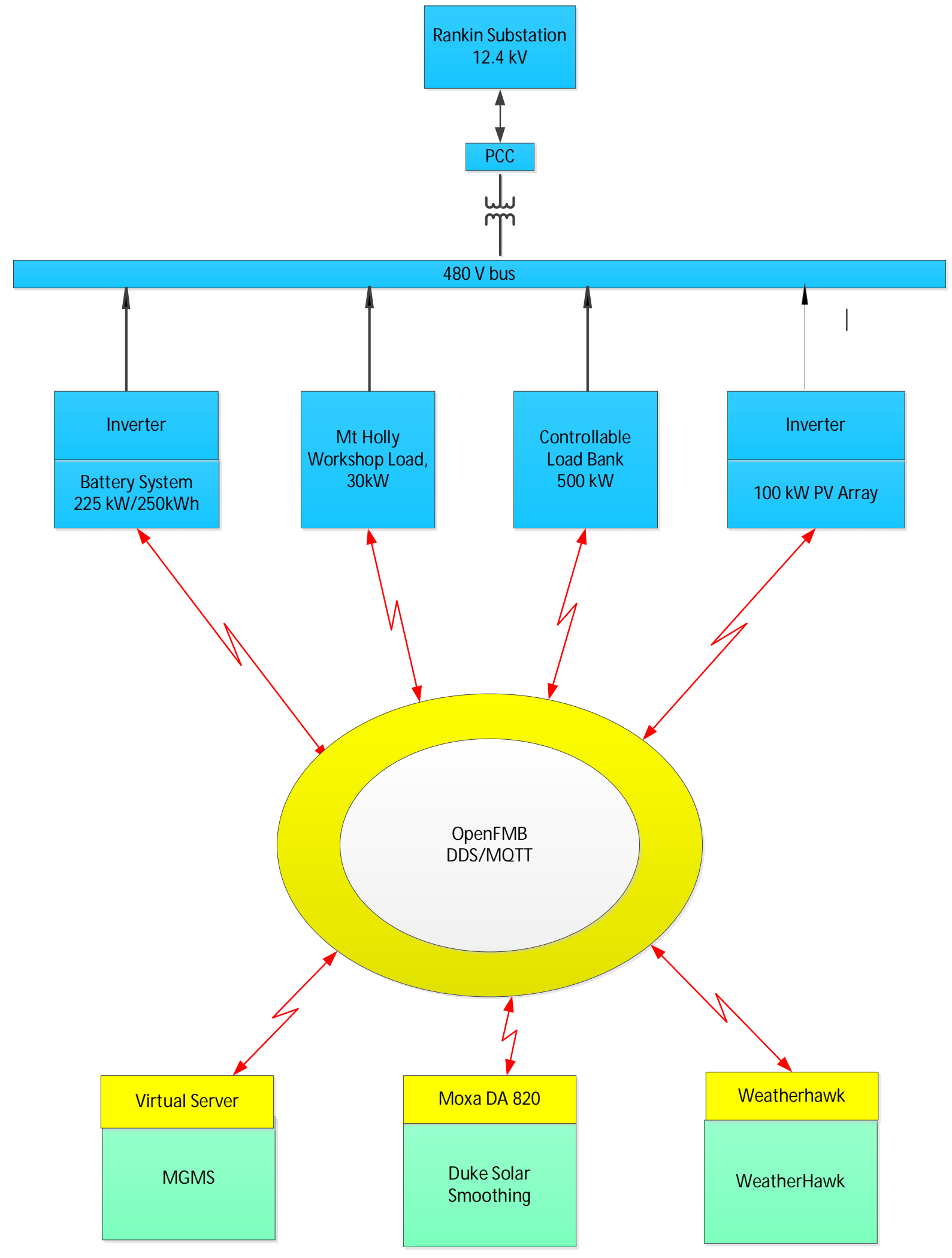

Figure 69: OpenFMB Architecture at Mt Holly 
The Siemens MGMS was configured to monitor elements of the Mt Holly microgrid as shown Figure 70: Mt Holly SCADA diagram. The Siemens MGMS was integrated with the OpenFMB Architecture with DDS/Web Services, and DNP/DDS Adapters.

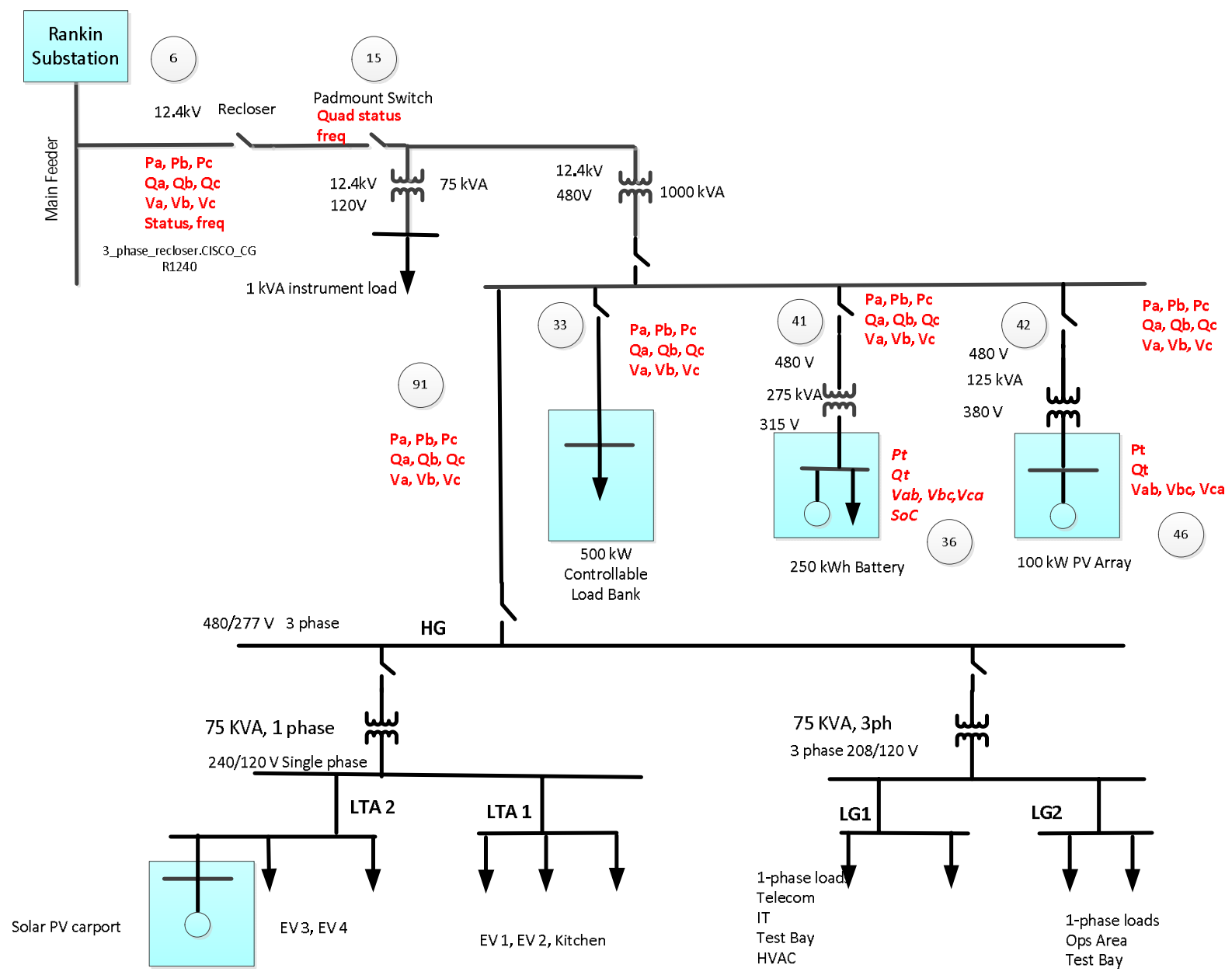

Figure 70: Mt Holly SCADA diagram

\subsection{Control Applications}

The Siemens MGMS was integrated using the OpenFMB Architecture with the real time control application developed by Duke. The Siemens MGMS included load and solar PV generation forecasting applications that are based on a similar day/pattern matching algorithm, in place of the applications described in Section 5. These applications produce the inputs for the optimal scheduling and dispatch of the Mt Holly renewable resources. The Siemens MGMS produces advisory day-ahead schedules for the next 24-36 hours, with typical examples show in Figure 71. These example schedules are for the 
interchange with the feeder at the point of common coupling, and for the desired state of charge of the BESS. The MGMS also produces mandatory dispatch instructions for the next 5-minute dispatch interval.

In the implementation at Mt Holly, the optimal schedules were produced which respected the constraint of no reverse power flow to the utility grid and were published as advisory schedules. A dispatch signal was sent to the BESS as the desired state of charge for the end of the next 5-minute interval. The mandatory dispatch signal is sent on a 5 -minute cycle (slow cycle). The signal was coordinated with the real time control application, which operates on a 100 milli-second cycle to adjust the setting of the load bank and the battery, to ensure that there is no flow from the microgrid back to the utility grid Feeder 1201, from the Rankin Substation within the 5-minute interval. This application ramped the BESS to achieve the desired state of charge at the maximum sustained rate of charge, and then "wiggles" the settings of the battery and the load bank at a 100-milli-second interval (fast cycle) to enforce the constraint of no backflow to the utility grid that may arise from fluctuations in the loads or solar PV generation within the 5-minute dispatch interval of the MGMS, as shown in Figure 72.

The Siemens MGMS was integrated with the Duke supplied applications for seamless, unplanned transition from grid connected to islanded operations. The Siemens MGMS detects the unplanned transition to islanded operations and suspends the joint optimization of utility grid and microgrid resources. It then will start optimization of microgrid resources if there are sufficient controllable resources within the microgrid to perform an optimization. In the case of the Mt Holly microgrid, an island microgrid optimization was not possible as the only controllable resource is the BESS, which is used to balance fluctuations in load and renewable generation. 


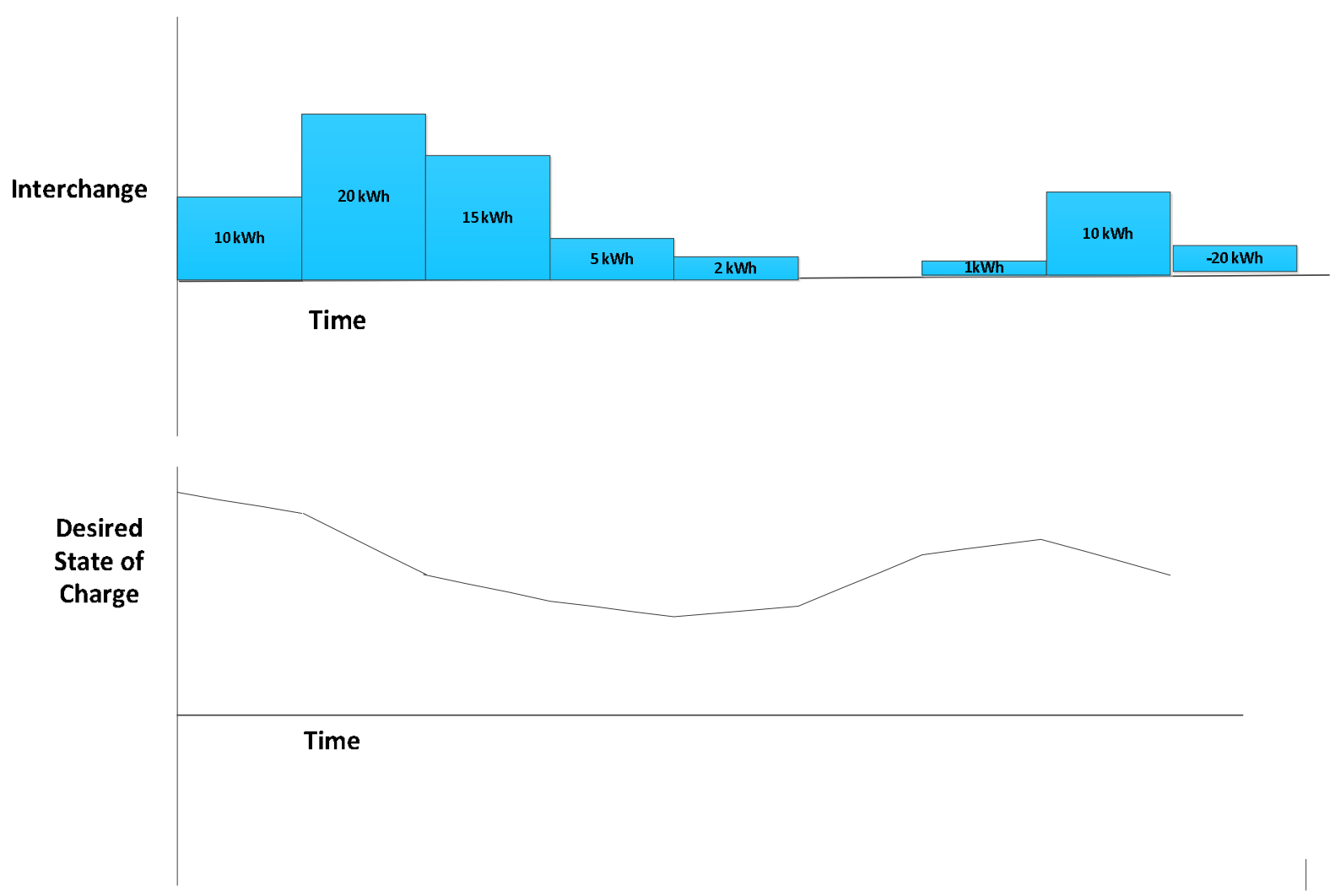

Figure 71: Optimal Advisory Schedules

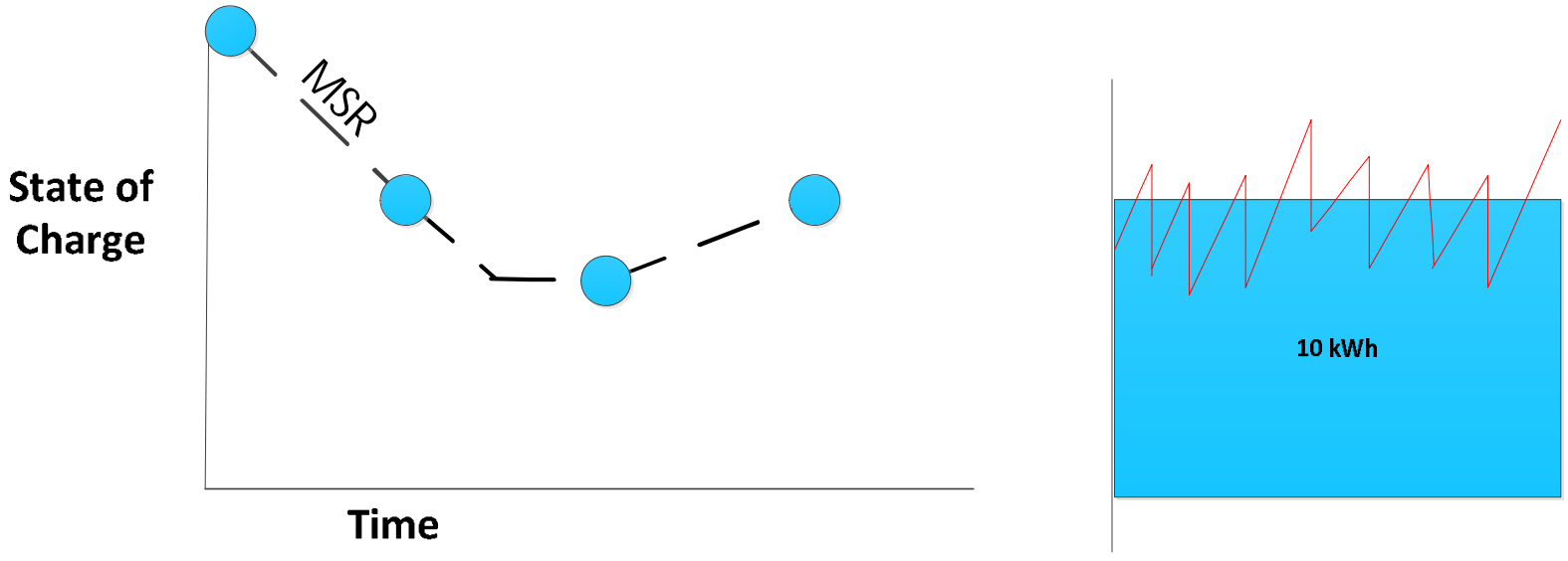

Figure 72: Mandatory Dispatch Signals

\subsection{Demonstration to Industry}

A demonstration was given to NREL staff and to industry stake holders on June 28, 2016 at the Duke Energy Training Center at Mt Holly North Carolina. The demonstration included a review of Mt Holly microgrid, a review of the project concepts, and a review of the OpenFMB Architecture. The demonstration included optimization of the microgrid when connected to the utility grid, a demonstration of the spontaneous transition of the microgrid from operating in grid-connected to islanded operations, 
and a planned transition from islanded back to grid-connected operations. The transition of the microgrid from grid connected to islanded operations was seamless as there was no detectable blink of the lights in the Mt Holly workshop. A sample of the operational data that was recorded in a data historian during the industry demonstration is shown in Figure 73.

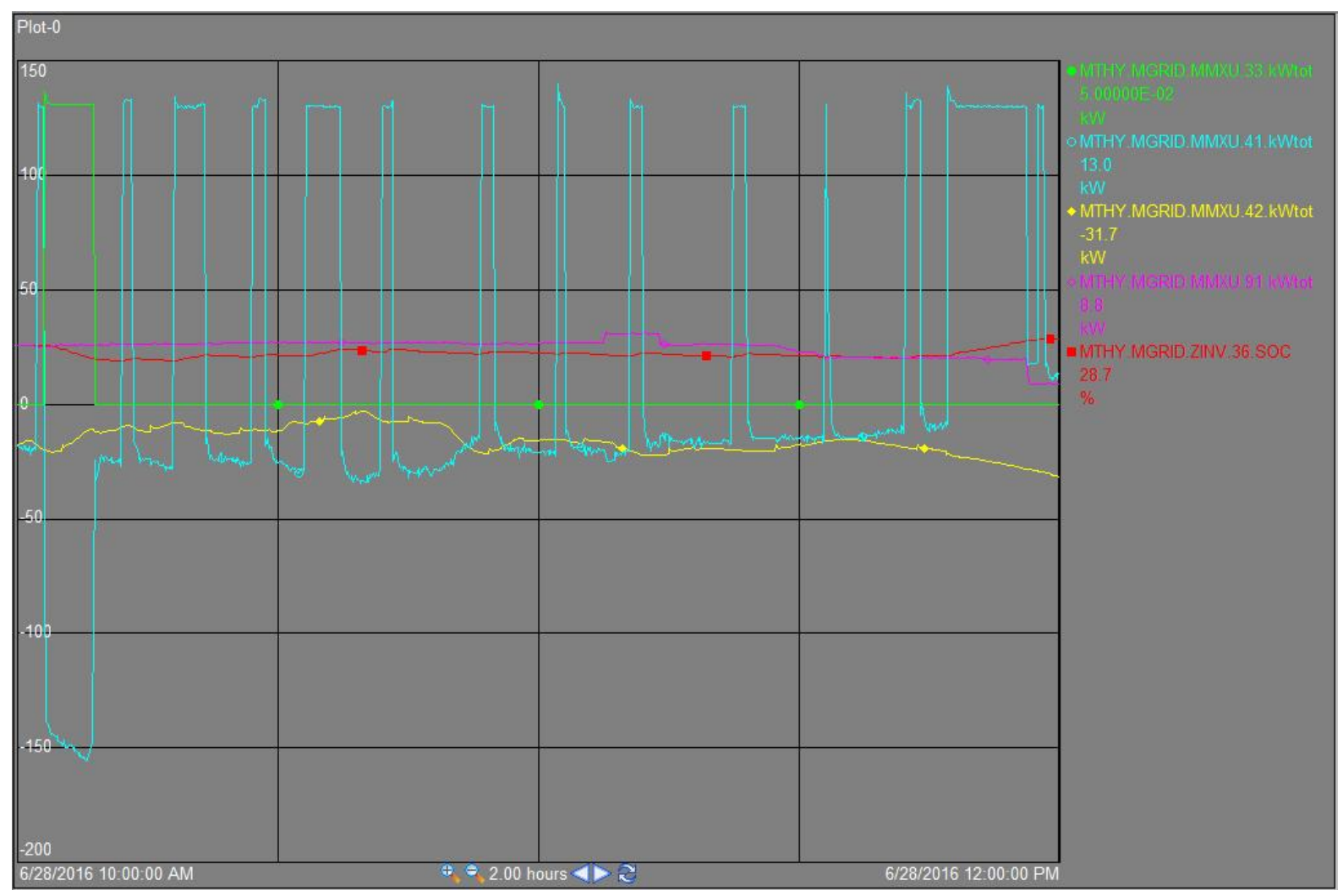

Figure 73: Mt Holly Microgrid Operational Data

A copy of the press release is included as Appendix A4.

\subsection{Test and Test Results}

Structured and unstructured tests were carried out at the Mt Holly microgrid. Testing of the OpenFMB was carried out using the test book [3], which thoroughly tested the publish/subscribe model for all communication paths shown in Figure 30. Testing included use of an implementation of the open 
standard DDS by RTI [8] for the transport layer of the OpenFMB Architecture. The use of the open source implementation of MQTT from the9] as a transport layer of the OpenFMB was also verified.

Testing of the integration of the Siemens MGMS with the OpenFMB architecture was also included in the test book. Results of the tests are recorded in the Test Results [4].

The testing at the Duke Energy Mt Holly, in the opinion of the project team, verified the ability an implementation of the OpenFMB Framework to integrate actual renewable components so that they can support the selected use cases. It also demonstrated an MGMS integrated with the OpenFMB architecture that can optimally schedule and dispatch the resources of a microgrid on both a day-ahead and an intraday basis, and that can also make these optimal schedules available to a hierarchical control system as envisioned in the project concept described in section 2 . The testing also validated the concept of locating applications at the edge of the utility grid to acquire and act on data locally. The testing verified the ability of the OpenFMB to support interoperability of multiple protocols, with the latencies required for monitoring, control, scheduling and dispatch of microgrid functions. 


\section{Field Demo at CPS}

\subsection{CPS Energy Microgrid at Joint Base San Antonio}

CPS Energy of San Antonio TX (CPS Energy) has a microgrid at the Joint Base San Antonio, a military facility for the US Army, Airforce, Navy, Marines, and Coast Guard. The microgrid includes a solar PV array of $20 \mathrm{~kW}$ and a $75 \mathrm{~kW} / 48 \mathrm{kWh}$ battery located in close proximity to the JBSA library which comprises the majority of the microgrid load which varies in the range $40-68 \mathrm{~kW}$ depending on the time of day and seasonal cycles.

The one-line diagram for the microgrid is shown in Figure 74. Superimposed on this diagram are circles with interior numbers that indicate telemetered points with quantities in the color red that indicate realtime data.

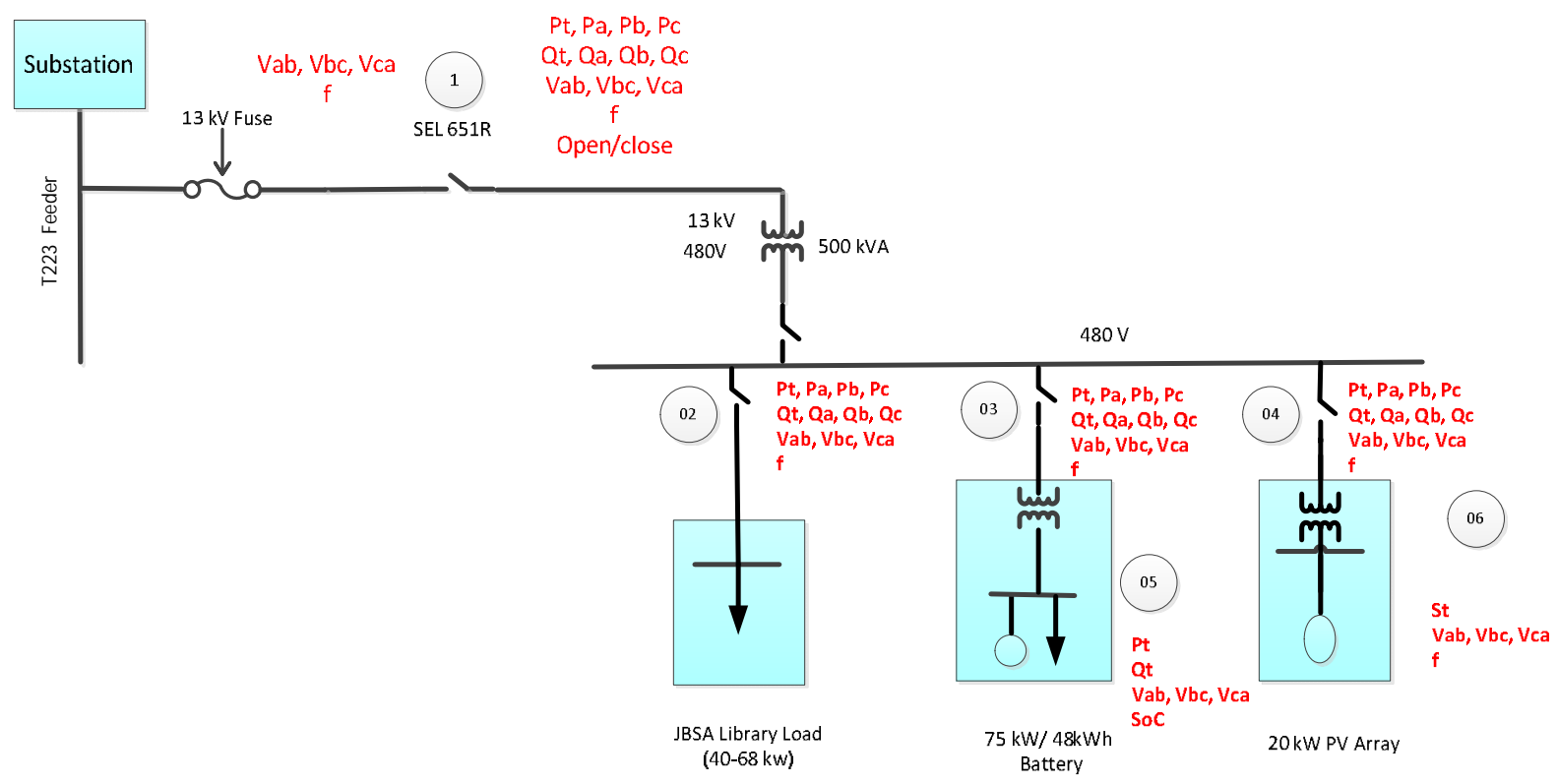

Figure 74 CPS Energy Microgrid at JBSA

\subsection{Monitoring and control of the JBSA Microgrid}

An implementation of the OpenFMB Architecture was set up at CPS Energy to monitor and control the resources of the JBSA microgrid as shown in Figure 75[1]. Schneider ION smart meters were used to measure the output of the Solar PV Array and the charging/discharging of the BESS. Import/Export at 
the Point of Common Coupling was measured from the SEL 651 Relay. The charging/discharging and state of charge of the BESS was additionally measured at the BMS as was the Apparent Power at the solar PV array. Real and Reactive flows and Phase and Line Voltages were measured as shown in Figure 74.

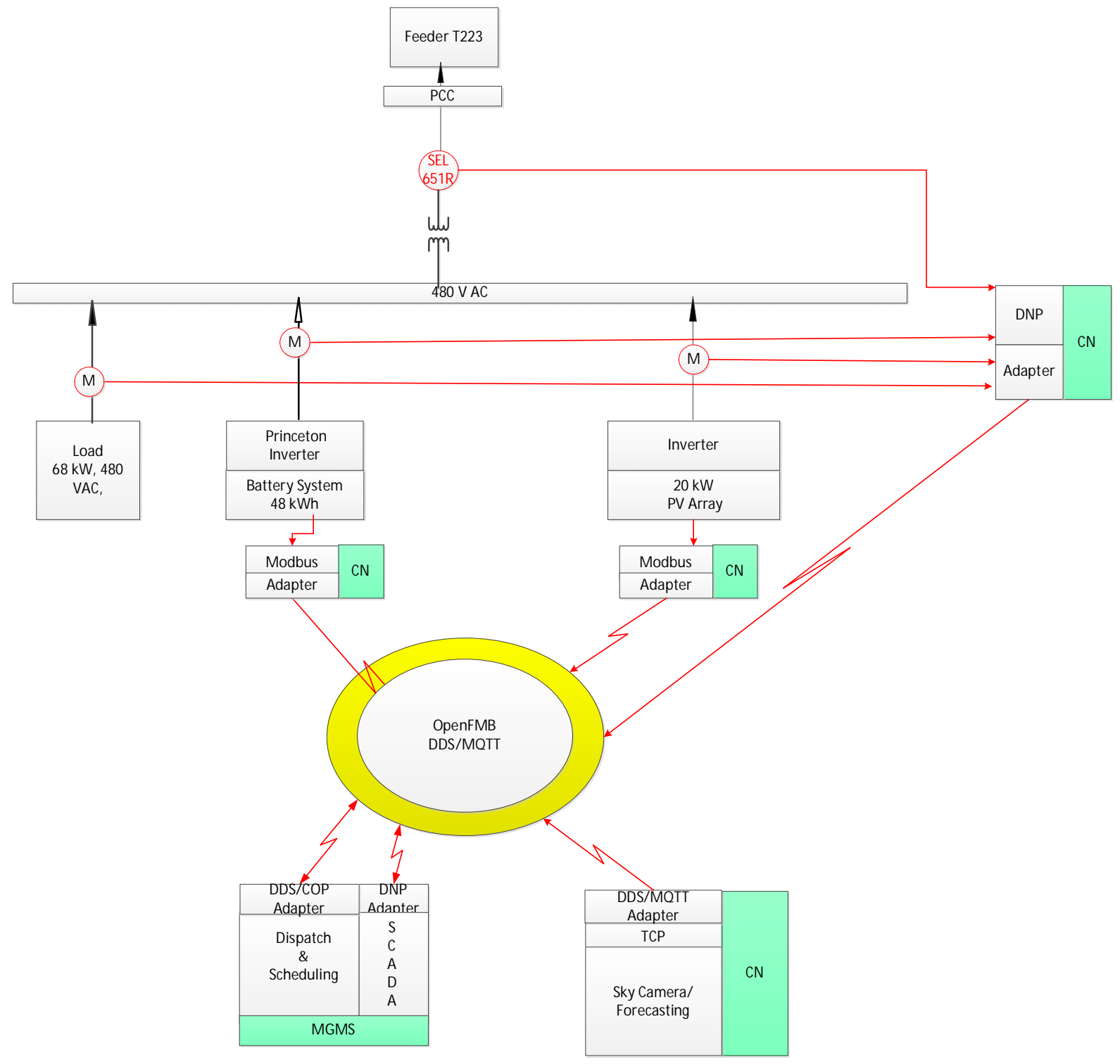

Figure 75: OpenFMB Architecture at JBSA

\subsection{Deployment of the OpenFMB}

The OpenFMB was deployed as shown in Figure 76Figure 75: OpenFMB Architecture at JBSA [1]. 


\subsubsection{Communications Nodes}

Two instances of the Siemens RuggedCom Utility Grade Layer 2/3 Switch and Router were used as

Communication Nodes for hosting the following adapters:

- DDS/Modbus for the BMS

- Modbus/DDS for the BMS

- $\mathrm{DDS} / \mathrm{DNP}$ for the BMS

- DNP/DDS for the BMS

- Modbus/DNP for smart meters

- $\quad$ DNP/DSS for the Solar PV Array Inverter (PowerHub)

- MQTT broker for the UTSA Forecasting Applications

Control of the BESS through the BMS was demonstrated in two steps. The BMS supports two native protocols: Modbus and DNP3. The project team initially used the Modbus protocol with an adapter to DDS during the demonstration to the industry. This was later changed to DNP3 as CPS plans a dual ported implement of the BMS, so that BMS data can be made available to both the CPS DMS (which uses DNP 3) and the Siemens MGMS.

\subsubsection{MGMS Server}

The MGMS sever ran on a Virtual Machine that was hosted on a CPS Energy CISCO Server in the JBSA substation. This server supported VMware software to create a virtual machine running the Redhat Linux operating system for the Siemens Spectrum Power 7 MGMS.

\subsubsection{Sky Tower and Imager}

The Forecasting applications were hosted on a Hardkernel Odroid2 and a MyRio computer from National Instruments. This software included processing of images from the sky camera, the forecast of loads, and Solar PV generation, and an MQTT Adapter that publishes the resulting forecasts.

The forecasting application also had a secure connection to the external internet to access weather forecasts.

\subsubsection{JBSA V-LAN}

A virtual LAN was set up for the OpenFMB at the JBSA microgrid. The DDS and MQTT were used as the transport layer. Optical Fiber and copper were used as the physical media. Wi-Fi was considered but not used due to cyber security concerns. 


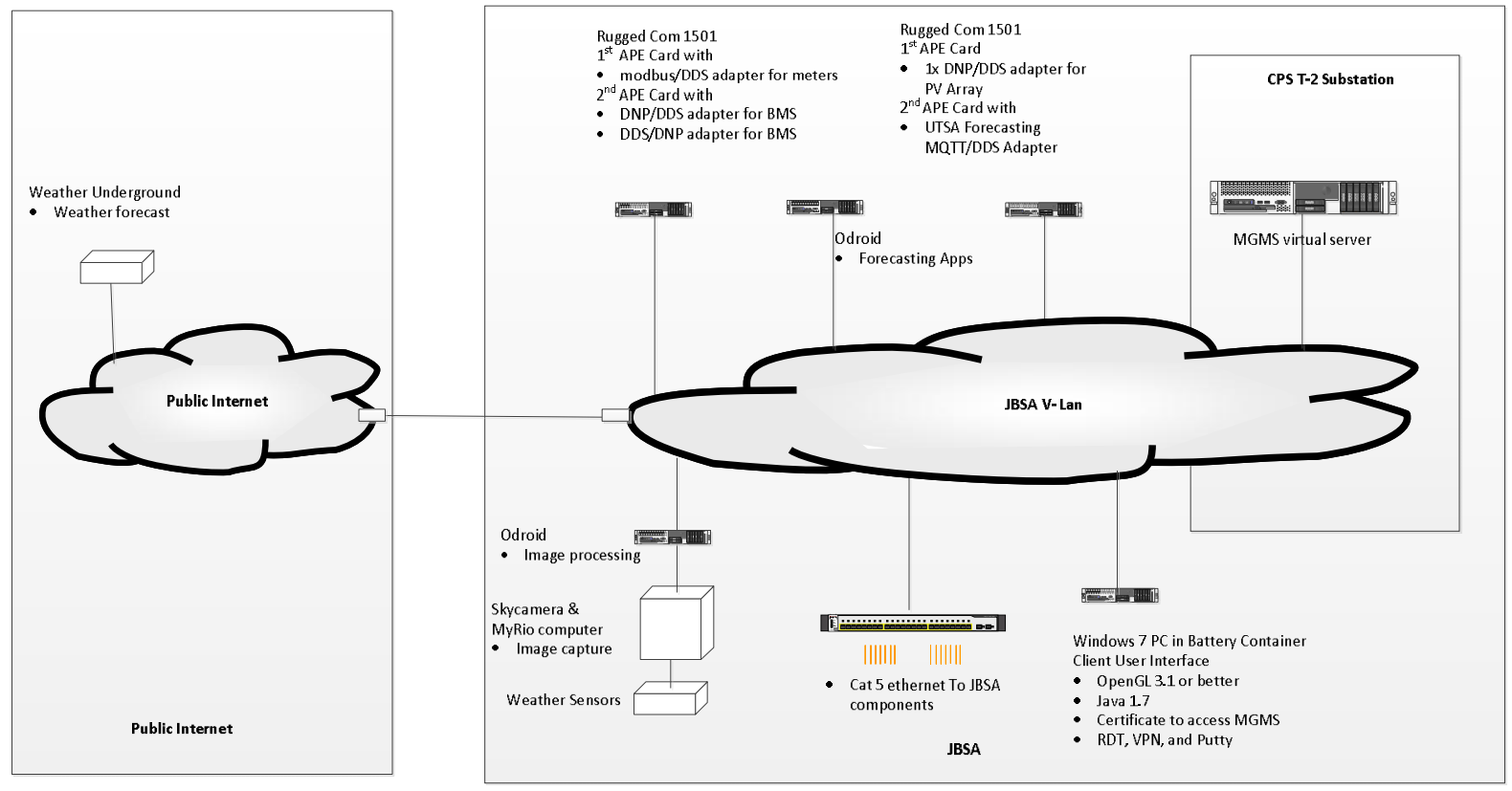

Figure 76: OpenFMB Deployment Diagram

\subsection{Sky Imager System Deployment at Fort Sam Houston Library}

The Fort Sam Houston Library location on Joint Base San Antonio presented several unique challenges for the deployment of the UTSA hardware and software that provide valuable insights into future considerations depending on site location. The ESIF building at NREL had in place the infrastructure necessary for an easy installation of both the Sky Imager and the Hardkernel Odroid C1+ that computed the machine learning Load/PV forecasts. Information was transferred using a Wi-Fi model using the NREL system. NREL also provided un-interruptible 120VAC power, ample Ethernet connectivity, and moreover existing on-site weather and irradiance data. Only cloud base heights $(\mathrm{CBH})$ had to be obtained from the ceilometer at the nearby Denver International Airport.

At Fort Sam Houston, the Sky Imager was deployed using an edge-computing approach with a wired Ethernet connection to address site - specific cyber security concerns. The Fort Sam Houston deployment included the Base Library building, solar arrays, inverters and the pod housing the battery (Figure 77). The need for accurate on-site meteorological observations prompted the decision to install a complete MET Station atop a 10-m guyed antenna-type tower. The concrete base of the MET tower was poured in early spring of 2016 and the tower itself was erected in April, 2016. At the tower base a Campbell Scientific Weatherproof Instrument Box was attached that contained a National Instruments MyRio computer, a transformer, backup battery, and the Hardkernel Odroid C2 for calculating the Load/PV forecasts. For the Fort Sam Houston Library deployment a complete weather station was deployed on a 
horizontal mast atop the MET tower that consisted of a WXT520 Vaisala Weather Transmitter, a pyranometer, and the electronics enclosure for the Sky Imager.

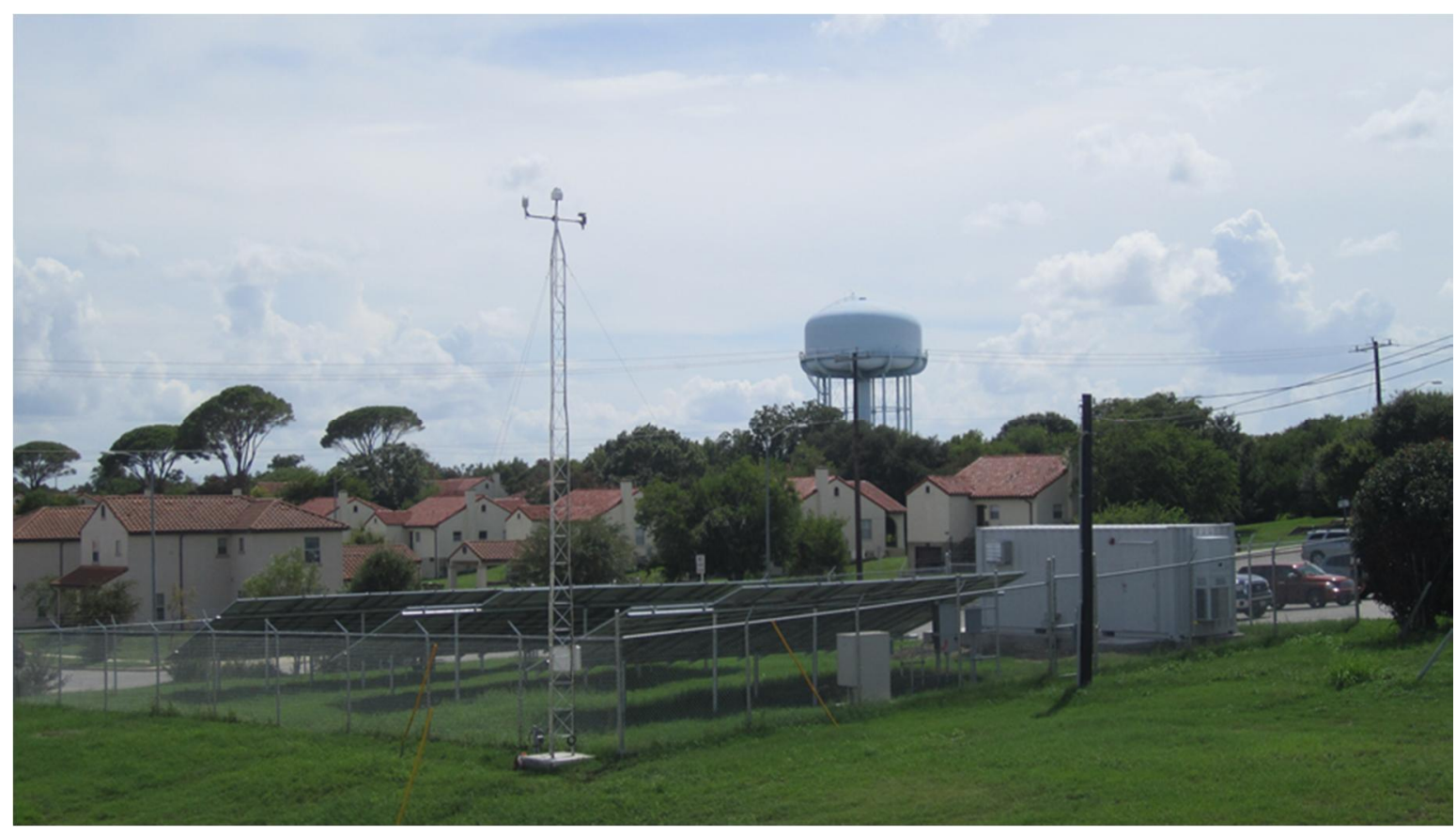

Figure 77: Location and layout of the Fort Sam Houston Library deployment

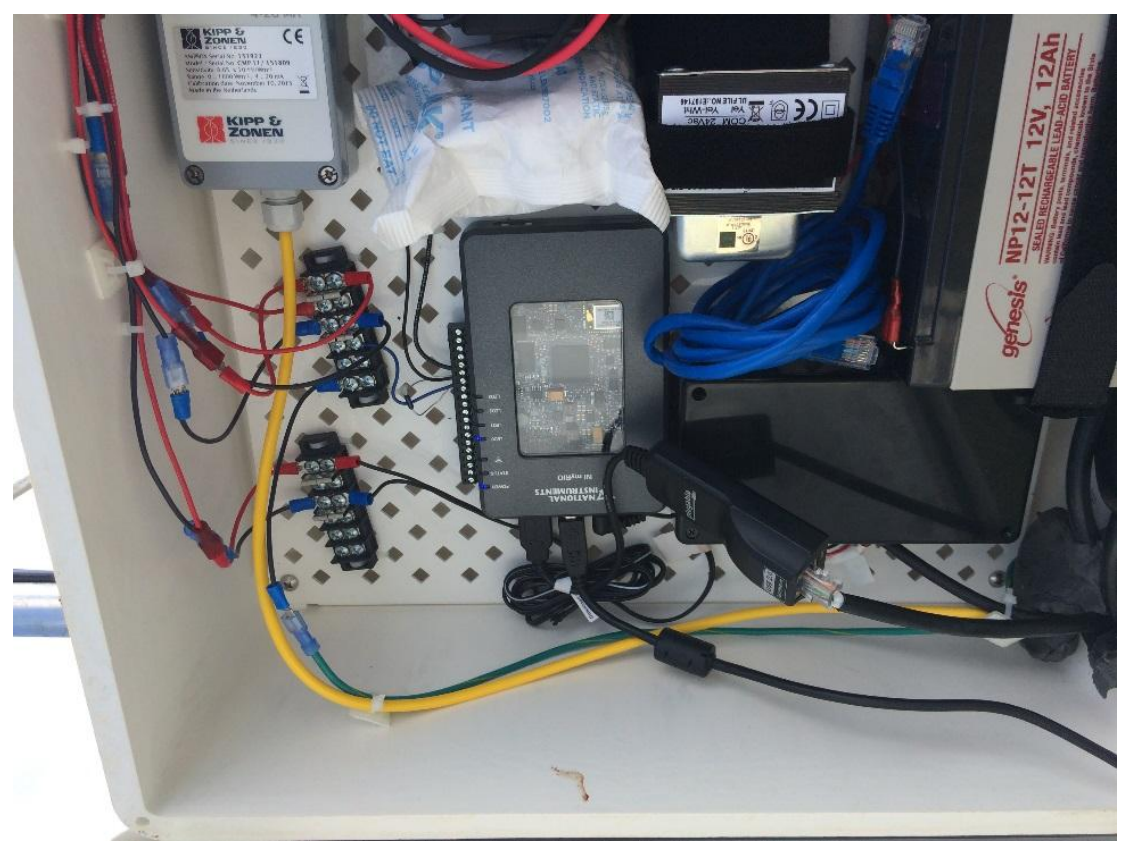

Figure 78: Internal Components of the Campbell Scientific Box with the battery, MyRio and transformer 
At the outset of the Fort Sam Houston Library effort, it was not fully appreciated that obtaining access to the military base for UTSA researchers would be a major obstacle to the effort. For safety purposes, it requires at least two people to lower the MET tower. Beyond the initial installation, access to the tower is required every few months to inspect the instruments and clean the surface of the plastic dome that covers the camera enclosure. Regular loss of power to the single board computers (SBC) as the battery went through its initial testing phase was also an issue. Initially the widely used Mosquitto MQTT broker was chosen for the UTSA software, but because of issues with the MyRio software, it was decided that HiveMQ would be a better choice. Finally, direct internet access to the computers from outside the CPS Energy network was available only through a WebEx session that severely limited deployment efficiency. The invaluable lesson learned from this deployment was that when initially transitioning hardware/software from a research environment to a production one, it is imperative to have both physical and cyber access to the equipment and software.

\subsubsection{Load forecasting and day-ahead PV production forecasting}

The micro grid management system (MGMS) gives an operation schedule for up to 16 hours (user selectable) by generating the 15-minute interval inputs for the grid operation during the optimized horizon. These inputs include load and generation forecasts, prices of imported power at PCC, and SOC at end of horizon and the outputs include desired flow at PCC, and set points (charge/discharge) of battery. Offline tests of the Fort Sam Houston Library have proven the large uncertainty of the data in terms of the PV and load pattern. For load forecasting (Section 5.3.1.3) the error could go up to $17 \%$ in terms of the 24-hour ahead case compared to intra-hour ahead forecast's error that is around 10\%. It was recognized that with the subscribed third-party weather forecasts (Weather Underground) at 1 hour intervals would increase the uncertainty of the interpolation from hourly scale to 15-min scale and diminish.

For PV forecasts, the uncertainty is even larger considering the lack of accessibility to solar-related inputs. The offline tests could easily get the solar irradiance information such as solar irradiance and produce a fairly accurate prediction for the day ahead horizon. The current deployment of PV forecasting at CPS Fort Sam Library uses only data available and provided by the MGMS (e.g., measured temperature) and the Weather Ground (e.g. forecasted temperature)- this did not include any projection of solar related input (e.g. the cloud cover 24 hours ahead). However, the UTSA forecasting approach can accommodate a third-party source (Weather Underground) for the weather forecast leading to an enhanced version of the current temperature based PV forecast. This approach uses the cloud cover level from the Automated Surface Observing System (ASOS). This Enhanced MGMS Forecast (with Cloud Cover) can utilize readily available information that predicts cloud cover at various elevations 24 hours ahead. The forecasting approach processes and categorizes the cloud cover level to sky clear (CLR), few clouds (FEW), scattered clouds (SCT), broken clouds (BKN), overcast clouds (OVC), and nil significant clouds (NSC). Plots that compare the cloud cover level to the PV production on Fort Sam for specific days are shown from Figure 79 to Figure 81As recognized by others, it is clear that there is strong correlation between the cloud levels and the PV production curve in all day types. For sunny days, a clear 
sky condition produces a smooth and normal PV production curve in Figure 79 while a cloudy day produces a very low level of PV production (Figure 80). The more irregular production in partly cloudy days further illustrate the contribution of the cloud cover level to the PV production pattern (Figure 81).

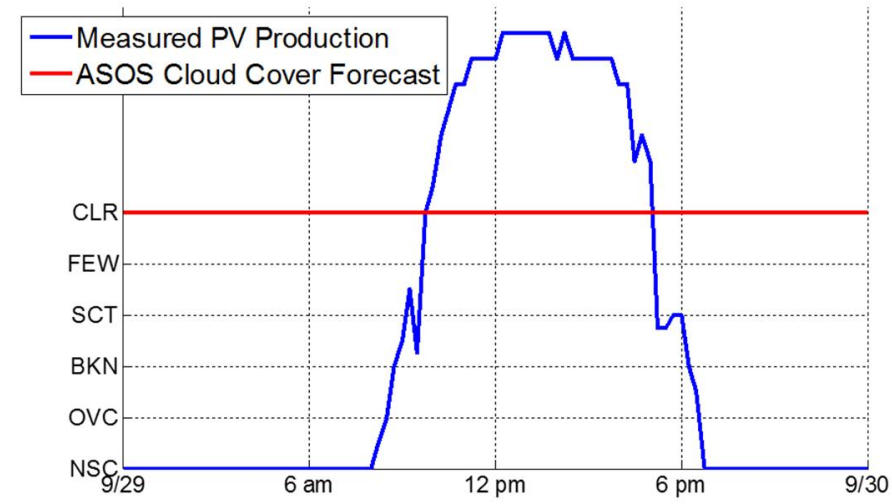

Figure 79: Comparison of measured PV production and ASOS cloud cover forecast for a partly cloudy day

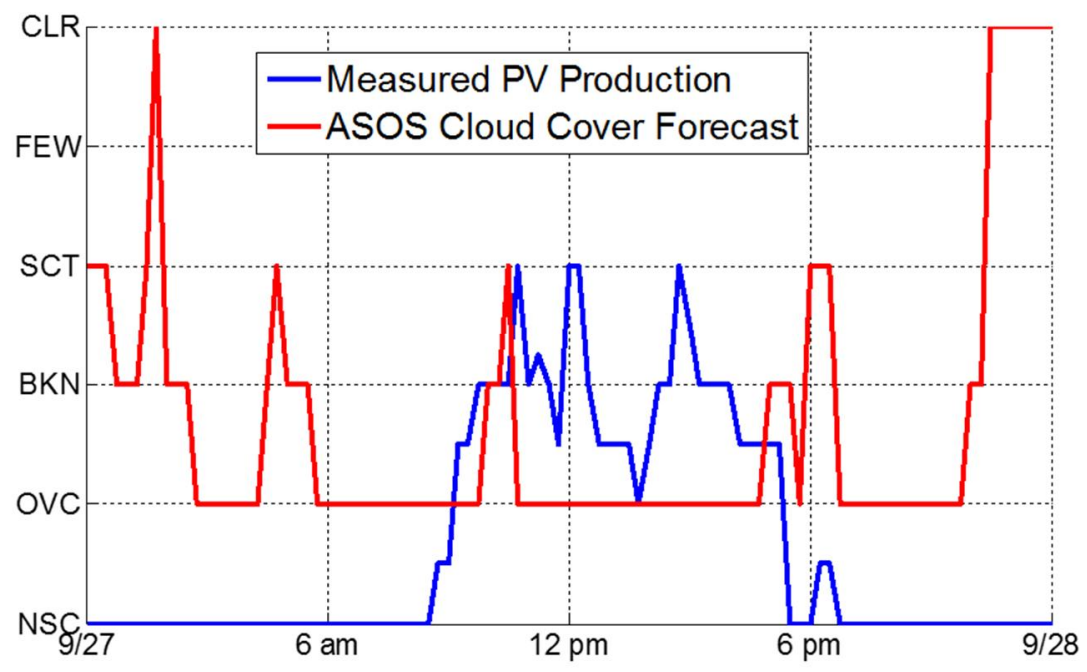

Figure 80: Comparison of measured PV production and ASOS cloud cover forecast for a cloudy day on Sep 27, 2017

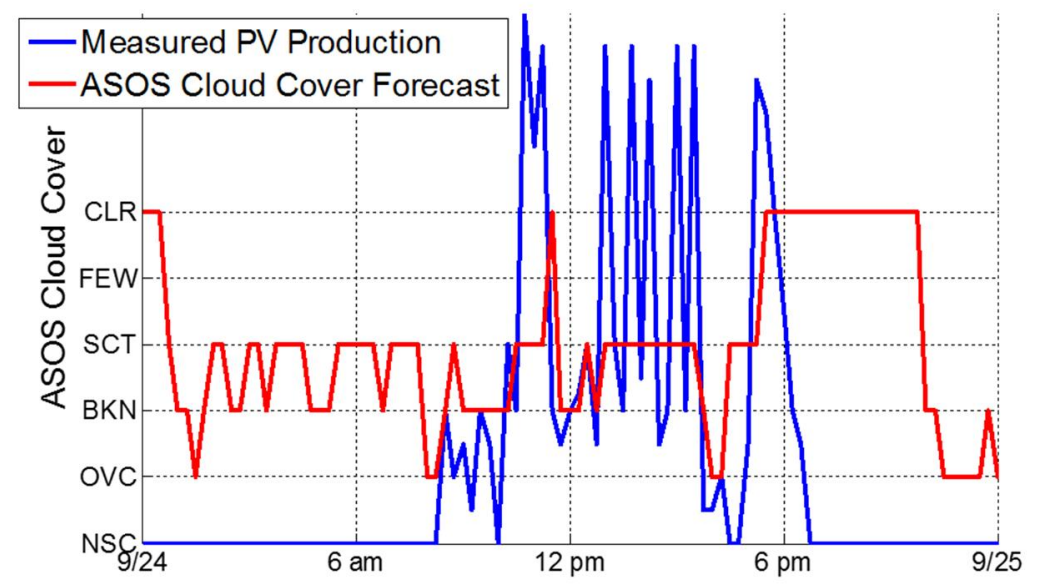


Figure 81: Comparison of measured PV production and ASOS cloud cover forecast for a cloudy day at Sep $24^{\text {th }}$

To demonstrate the effectiveness of the enhanced version of PV forecasting, one week of mixed day types are tested. The week spans from September 23 to September 29, 2016. The 24-hour ahead forecasts are performed based on two input sets: the temperature (PV Forecast), and the temperature with the solar related information such as the cloud cover levels (Enhanced PV Forecast). Results of different weather conditions are shown in the following figures. From the observations of the forecasts, the days with partly cloudy weather conditions are very hard to be predicted, as show in Figure 82. The input with temperature only tends to over predict while the added cloud cover information improves the model prediction in an observable manner. The consistency of the irregular PV production seems to create very large uncertainty comparing to the purely sunny days. Meanwhile, the cloud cover information could be very helpful with a mixed pattern of sunny days and cloudy days, as shown in Figure 83. For cloudy days, the cloud cover information could help to improve the forecast by avoiding the impacts from the historical information. One example shown in Figure 84 illustrates the significant improvement with cloud cover information compared to the model without cloud cover information. The NMAE results show significant improvement for forecasting of the connected mixed days including cloudy, partial cloudy and sunny days, as shown in Table 7.1.

Table 12: Fort Sam Houston PV Forecasting NMAE under the complex weather situation

\begin{tabular}{c|c|c}
\hline $\begin{array}{c}\text { Complex } \\
\text { Weather }\end{array}$ & $\begin{array}{c}\text { Temperature } \\
\text { based PV Forecast }\end{array}$ & $\begin{array}{c}\text { Enhanced PV } \\
\text { Forecast }\end{array}$ \\
\hline 1-hour ahead & $11.42 \%$ & $11.16 \%$ \\
\hline 24-hour ahead & $33.13 \% \%$ & $16.77 \%$ \\
\hline
\end{tabular}

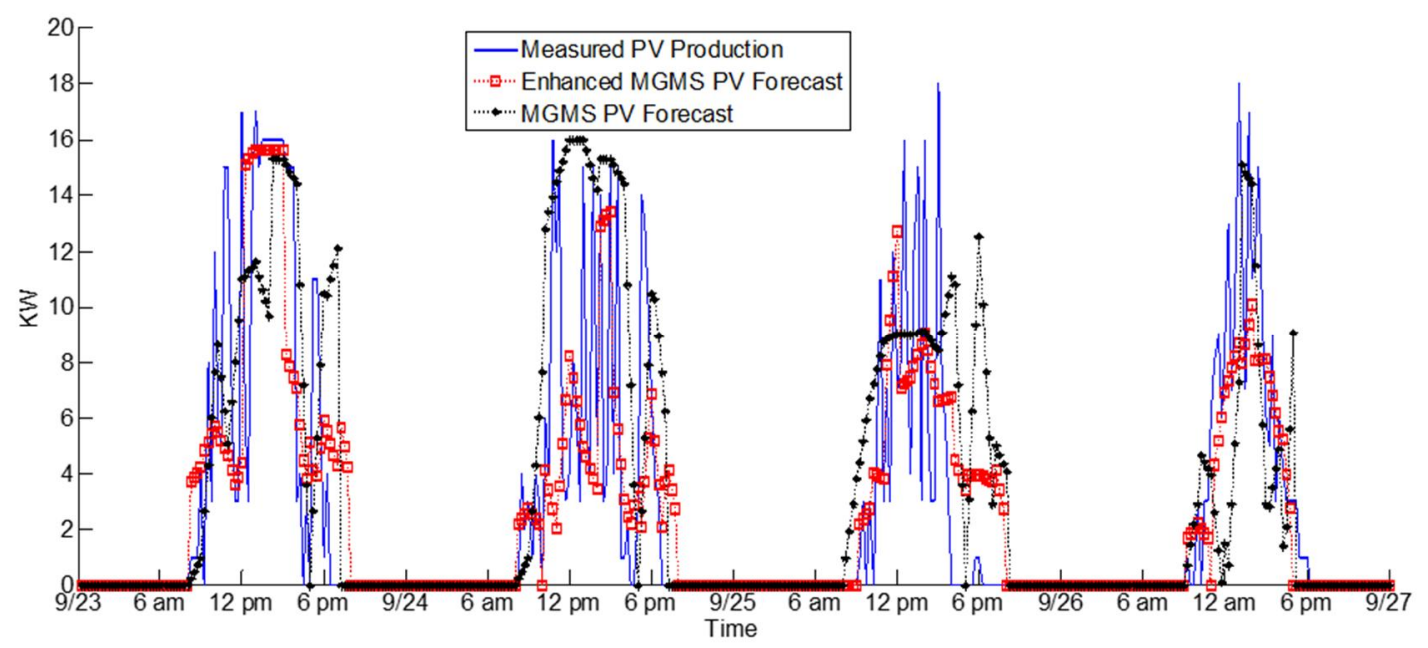

Figure 82: Consistent but partially cloudy days 


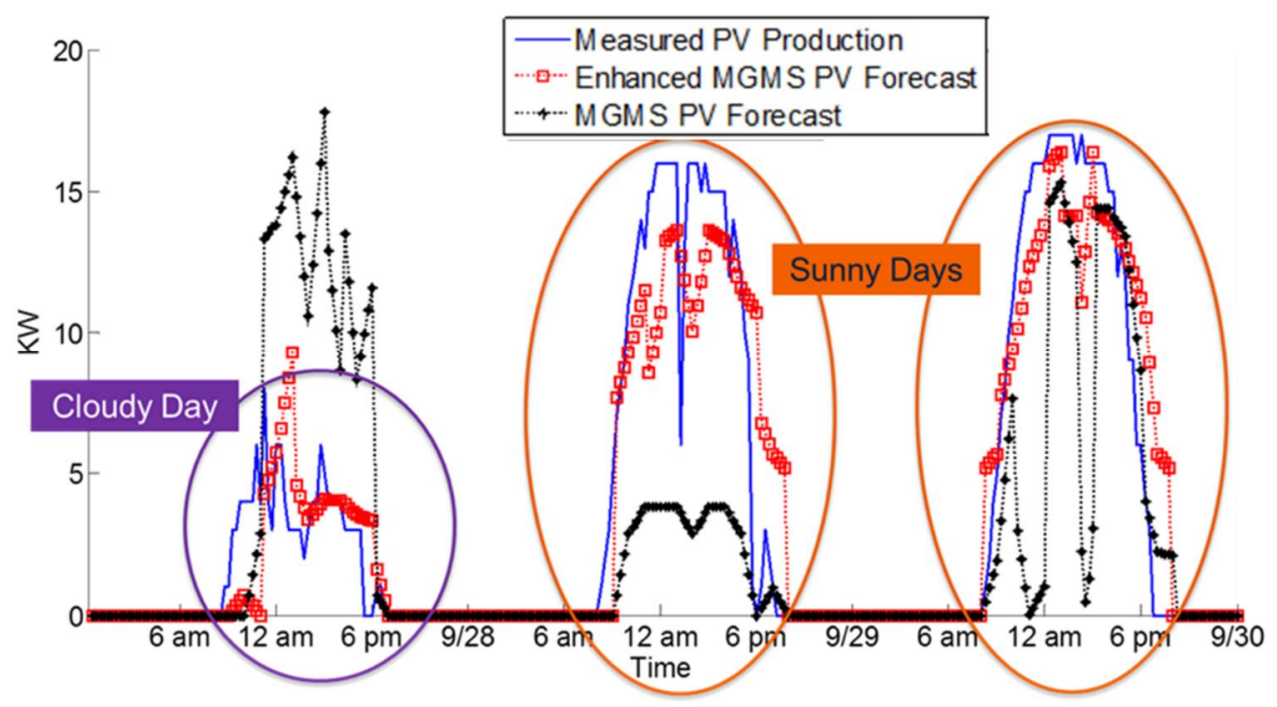

Figure 83: Inconsistent days with mixed cloudy and sunny days

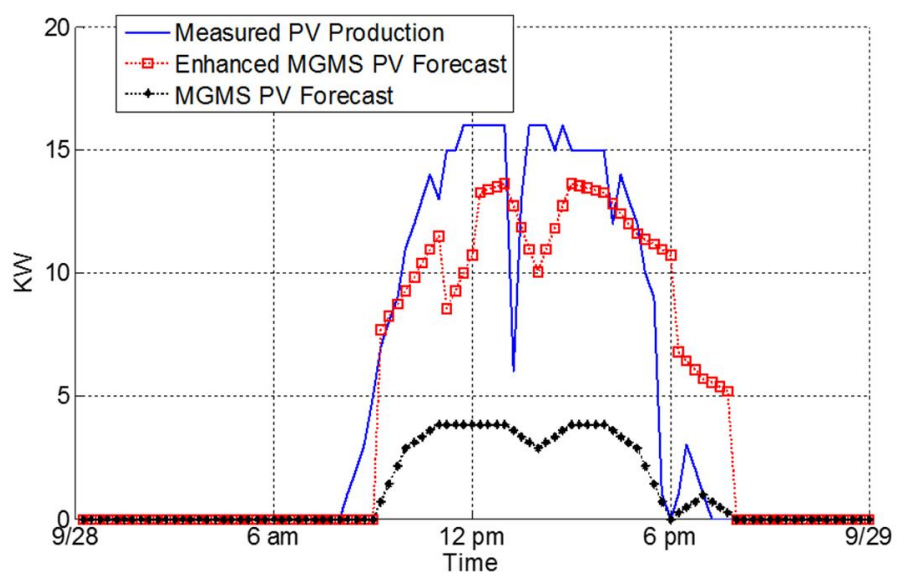

Figure 84: Cloudy Day with the previous day as a sunny day

\subsection{Demonstration to Industry}

A demonstration was given to NREL staff and to industry stake holders on September 27, 2016 at the Joint Base San Antonio. The demonstration included a review and inspection of the JBSA microgrid, a review of the project concept, and a review of the OpenFMB Architecture. The demonstration included monitoring of the JBSA microgrid and remote control of the BESS through the BMS.

\subsection{Test and Test Results}


Structured and unstructured tests were carried out at the JBSA microgrid. Testing of the OpenFMB was carried out using the test book [2], which thoroughly tested the publish/subscribe model for all communication paths shown in Figure 36. Testing included use of an implementation of the open standard DDS by RTI [4] for the transport layer of the OpenFMB Architecture. The use of the open source implementation of MQTT as a transport layer of the OpenFMB was also verified.

Testing of the integration of the Siemens MGMS with the OpenFMB architecture was also included in the test book. Results of the tests are recorded in the Test Results [3].

The testing at the CPS Energy JBSA Microgrid, in the opinion of the project team, verified the ability an implementation of the OpenFMB Framework to integrate actual renewable components so that they can support the selected use cases. Optimal scheduling and dispatch of the resources of a microgrid was not possible due to the relatively small size of the battery (48 kWh) compared to the JBSA library load of 40$68 \mathrm{~kW}$. The load forecasting and solar PV forecasting applications were demonstrated with good accuracy as measured by common error statistics, as described in Section

The load forecasting capability demonstrated as part of the INTEGRATE effort extends traditional linear regression or persistent approaches to include Artificial Neural Network and Support Vector Machine methods that are specifically designed for on-line forecasting. The adaptability of the models for incorporating null values in the inputs, the change of the input types, the handling of outliers (e.g. the negative values in PV productions), and the interpolation for 15-minute resolution forecasts contribute collectively to a more refined, more accurate forecast prediction.

Load forecasts must consider the intended use of the buildings and the building operations and control maintenance schedule as integral inputs. Many different building attributes will influence the load forecasts and predictability (e.g., building age, size, use, occupancy, etc.). Occupant behavior can play a significant role in the quality of the predictions

PV solar forecasting approaches continue to evolve but vary significantly depending on local meteorological conditions, the quality and availability of data on cloud cover variability, and seasonal temporal variabilities in weather over 1-hour, 24-hour and 36-hour intervals. Highly variable weather patterns reduce the accuracy of the forecasts considerably because of limitations in the modeling process.

Integrating weather and cloud forecasts into the data published by the MGMS improve solar PV forecasts considerably, even when weather patterns change over short durations.

Site protocols for operations and maintenance can have significant impacts on data quality, hence forecast errors, and on the efficiency of operations. Site operations and access should be recognized and understood early in the planning process to accommodate potential concerns as part of the planning process. 


\section{Standards}

\subsection{Standards Used in the OpenFMB Framework}

The OpenFMB Framework and Reference Architecture includes a set of standards with recommendations on how to deploy them for specific implementations. A short summary of the standards appears below, organized by topic area.

\subsubsection{Connectivity Standards}

These standards apply to the connections to the Communications Nodes of Section 4. A Communication Node will include the following ports to allow connection to other power system management equipment:

- $\quad$ RS-232 (serial communications port)

- RS-485 (TIA/EIA serial port)

- USB (Universal Serial Bus)

- 8P8C/RJ45 (Ethernet plug)

- Fiber Optic (FDDI ANSI X3T12)

- $\quad$ Power Line Carrier (PLC)

\subsubsection{Wireless}

These standards apply to the transfer of information between points that are not connected by an electrical conductor. The idealized Communications Node of Section 4 includes support for the following:

- Cellular (4GLTE, 3G LTE, GSM, GPRS)

- Bluetooth (IEEE 802.15.1)

- $900 \mathrm{MHz}$ Radio

- RF ISM

- GPS (Global Positioning Satellite)

\subsubsection{Form Factors}

Communications Nodes may be deployed in a variety of environments, ranging from climate controlled data centers, to harsh environments in electrical substations. Communications Nodes are available in a variety of form factors including:

- IEEE 1613 (compliant enclosures)

- NEMA 4+ (Ruggedized Enclosure)

- 19-inch Rack (IEC 60297, EIA 310-D) 


\subsubsection{Standards Protocol}

The OpenFMB Framework calls for support of a number of protocols that are either widely used or available as part of the Internet Protocol Suite of standards and as shown in Section 4, Table 1, which is repeated below:

\begin{tabular}{|c|c|c|c|}
\hline Layer & OSI Model & TCP/IP & Protocols \\
\hline 7 & Application & \multirow{3}{*}{ Application } & \multirow{3}{*}{$\begin{array}{l}\text { HTTP, Telnet, SMTP, DHCP, DNS, TLS, SSH, } \\
\text { SSL, SNMP, XML, DNP, C12, REST, MQTT, } \\
\text { DDS, AMQP, CoAP, Modbus, ICCP, OPC }\end{array}$} \\
\hline 6 & Presentation & & \\
\hline 5 & Session & & \\
\hline 4 & Transport & Transport & TCP, UDP, DCCP \\
\hline 3 & Network & Internet & IPsec, IPv4, IPv6 \\
\hline 2 & Data Link & \multirow{2}{*}{$\begin{array}{l}\text { Network } \\
\text { Interface }\end{array}$} & \multirow{2}{*}{$\begin{array}{l}\text { IEEE 802.3, 802.11, 802.15.4, 802.16, Bluetooth, } \\
\text { MAC, PDCP, RLC }\end{array}$} \\
\hline 1 & Physical & & \\
\hline
\end{tabular}

Table 2: Standard Protocols

\subsubsection{Internet of Things (IoT) Standards}

The OpenFMB Framework includes models and concepts that also appear in the Industrial Internet Consortium Reference Architecture (IIC RA) [1]. This reference architecture provides guidance for the development of systems, solutions, and application architectures, such as the OpenFMB. The OpenFMB includes the publish/subscribe pattern with the following standard protocols:

- MQ Telemetry Transport or MQTT (ISO/IEC stand PRF 20922)

- Data Distribution Services or DDS (OMG)

- Advanced Message Queuing Protocol (OASIS Standard)

\subsubsection{Semantic Data Model Standards}

The OpenFMB Framework calls for an information model to be used to define the semantics of data transfers. The OpenFMB Framework, in principle, could be based on one or more semantic information models. These models include:

○ IEC Common Information Model or CIM (IEC 61970, 61968, 62325) [2],[3],[4]

- IEC 61850 semantic data model[5]

- Other Enterprise Semantic Models

This project used profiles or subsets of the IEC CIM UML Model that were defined as part of the SGIP OpenFMB Priority Action Plan (PAP). Project Team members participated in the PAP working group that defined the profiles that were used to support the SGIP use cases, and incorporated all data structures needed to support the NREL Project use cases.

\subsubsection{Cyber Security Standards}


The OpenFMB Framework calls for the use of common accepted cyber security standard such as the Common Criteria [6], and the various Federal Information Processing Standards [7]. They cover an integrated security policy for physical plant, hardware, software, and communications. Common security activities called out are:

- Security monitoring

- Security auditing

- Security Policy Management

- Cryptographic Support

The scope of this project called for adopting security measures required to support the demonstration in a controlled environment at the NREL ESIF, and the Duke Energy Mt Holly Training Facility. The third demonstration was at a CPS Energy microgrid facility located on the JBSA which included addition security procedures for access to and operation on the military base. Advancements and demonstration of the efficacy of these security standards was not included in the project scope.

\subsection{Relation to Parallel Work at SGIP, NAESB}

\subsubsection{SGIP}

This project was executed in parallel with other related projects at SGIP and NAESB. The SGIP created a priority action plan (PAP) project to define the OpenFMB Framework. Members of this NREL project also participated in this PAP project and contributed to the definition of the use cases and the definition of the CIM profiles for the Global Data Space publish/subscribe design pattern.

The SGIP also hosted a demonstration of a simulation of the OpenFMB at their November 2015 Annual Meeting in New Orleans. A prototype of the OpenFMB was demonstrated using a software simulation of a synthetic microgrid that was distributed to various processing platforms of the demo participants. The SGIP PAP project included the creation of a repository for OpenFMB materials that are now available under the terms of an Apache License. An announcement of the repository was made at the SGIP Annual Meeting on Nov 8, 2016 along with a demonstration of the software available in the repository.

\subsubsection{NAESB}

The North American Energy Standard Board (NAESB) created a project to document the OpenFMB Framework as a Model Business Practice. Members of the NREL project participated on the team that drafted the Model Business which was balloted and approved in March 2016. The results are available through NAESB as Model Business Practice RMQ.26, OpenFMB [8].

\subsection{Future Standardization Work}

The SGIP has reached out to the IEC Technical Committee 57 Power System Management and Associated Information Exchange to start a project to incorporate the OpenFMB into IEC standards. As 
of the date of this report, discussions are still underway to define a possible project and work procedures to make progress toward this goal. 


\section{Lessons Learned}

\subsection{Technological Readiness Levels}

NREL ESIF has a classification of Technological Readiness on a scale with technological readiness levels 1 through 8 . The scope of this project called for advancing the project technology to Technological Readiness Level 7, defined as:

"TRL-7: System prototype demonstration in an operational environment: This represents a major step up from TRL-6. It requires the demonstration of an actual system prototype in an operational environment, such as in a light duty vehicle on the road"

The validation of the project concept and the OpenFMB Architecture at NREL ESIF and the demonstrations in the ETO Training Center at Duke Energy and at the CPS Energy microgrid at JBSA indicate the project concept and OpenFMB are at Technological Level 7. The UTSA Forecasting Applications were assessed at TLR-7.

The next level in the scale is TRL-8, defined as:

"TRL-8: Actual system completed and qualified through test and demonstration: Technology has been proven to work in its final form and under expected conditions. In almost all cases, this TRL-8 represents the end of true system development"

The Siemens MGMS is an off-the shelf product which is at TRL-8. The remaining work to get the project concepts to Technological Level 8, is in the area of enhancing Cyber Security and Remote Manageability, which the project team sees as promising field for future work. This work is necessary before transferring implementations into a more exposed utility production environment.

\subsection{Battery Management Systems}

Battery Energy Storage Systems (BESS) have a number of operational modes that are described in state charts which show the states and the allowed transition between states. Figure 85shows an example chart for the BMS system for the Erigo Eagle Picher battery at NREL. Other battery suppliers have similar, but different state charts describing operating modes and allowed transitions. This lack of standardization presented a challenge in the describing the global data space for publish subscribe model of the OpenFMB. 


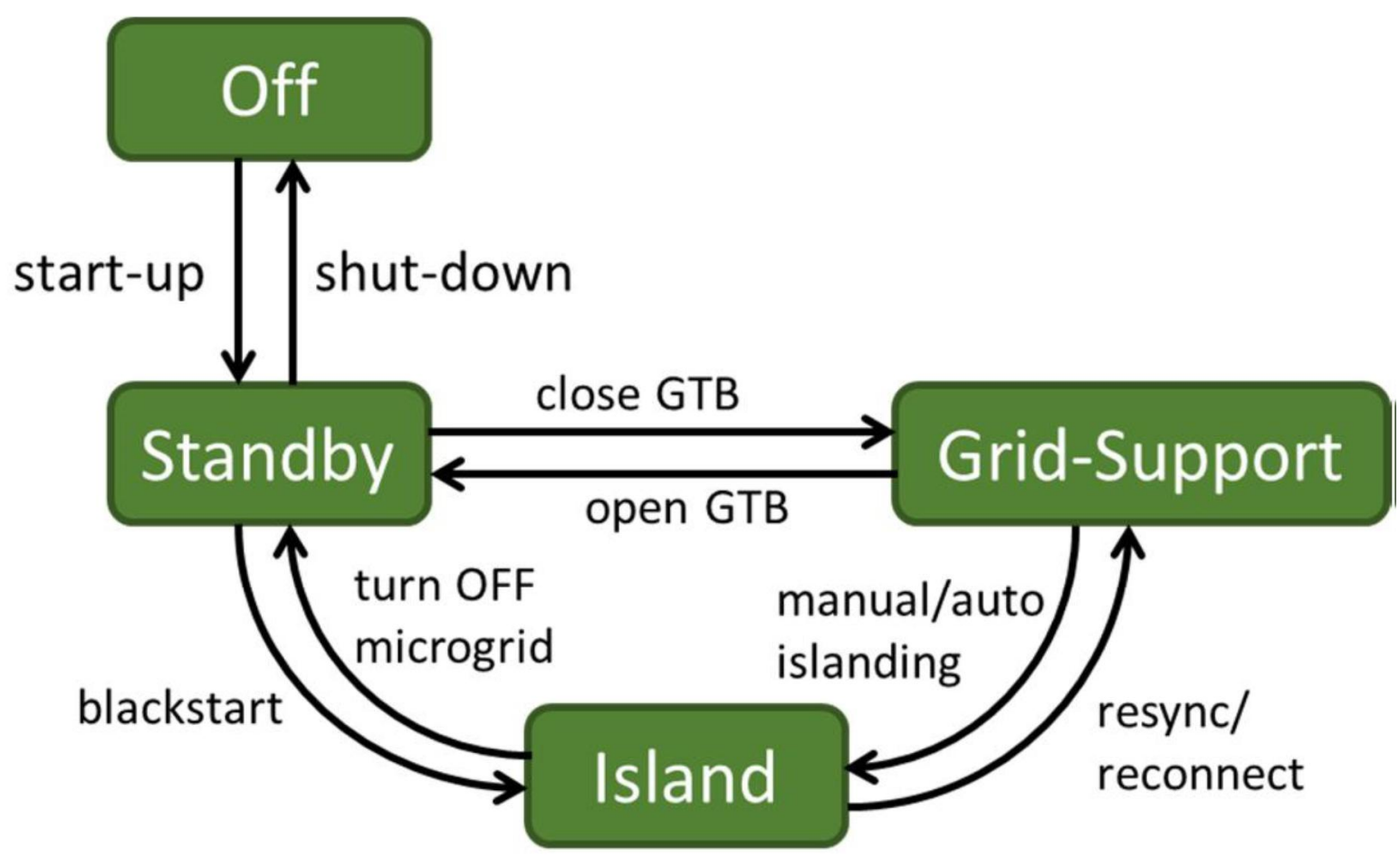

Figure 85: BMS State Chart

The problem will need to be addressed by the battery vendors, perhaps by developing an agreed standard interface model that each vendor can either adopt or provide a mapping to their proprietary implementations. The MESA Standards Alliance has a draft specification for utility scale energy storage systems that may provide a mechanism that battery vendors can adopt.

\subsection{Requirement for Remote and Physical Access}

The project teams required access to the various sites during the development of the projects, both physical access and secure remote access. Getting this access required approval from several different parts of the various organizations and included mandatory background checks and training, as is required by NERC Critical Infrastructure Protection standards, individual utility regulations, and NREL requirements. Access to the JBSA required additional applications and approval.

This process took longer than initially planned. The lesson learned is that this process should be started very early in the project to avoid project delays. 


\subsection{OpenFMB Management}

The OpenFMB presents a challenge in the area of system management due to its distributed nature. As OpenFMB runs on multiple CNs at the edge of the grid, manual installation of periodic software revisions, updates, and patches at multiple remote sites by a technician is uneconomic and is not an option. The OpenFMB Architecture calls for remote management of software. The NAESB specification calls for the use of the AMQP protocol for remote installation of binaries. The project team encountered the need to work on-site in some cases for work that could be done with remote management.

Demonstration of remote management of the OpenFMB was not in the scope of this project. However it is an issue that will need to be resolved prior to rolling out the concept on a large scale.

\subsection{Role of Virtualization}

An idealized communication node allows for the hosting of some of the OpenFMB CN functions in a virtual machine. In the demonstrations at Duke Energy and CPS Energy, virtualization software (Fusion 6) from VMWare was used to create a virtual machine that hosted the Siemens MGMS application. The implementation at NREL included testing of both a physical server and a virtual server, with successful results. Also, during the development phase of the forecasting applications, UTSA used remote servers in conjunction with local CNs during program development.

This successfully experience in development and testing leads to the conclusion that it is possible to deploy the OpenFMB with MGMS applications hosted in "the cloud" with I/O and adapter functions hosted locally in a $\mathrm{CN}$ provisioned with communications of sufficient reliable bandwidth to the provider of the cloud services.

A microgrid that is managed with cloud based controllers may suffer degradation of its ability to provide highly reliable power to critical loads during a system wide event, depending on the effect that the system wide event has on both the supply of power to and the communications paths with the physical servers that host the cloud services. The pros and cons of such an implementation would need to be assessed with a reliability study at the time of detailed design of the microgrid.

\subsection{Cyber Security}

The implementations of the OpenFMB were deployed on Virtual LANs set up inside the Enterprise IT infrastructure at NREL, CPS Energy, and Duke Energy. Cyber and physical security were provided at the 
enterprise level and by creation of a segregated virtual LAN for the microgrid control system. Access to the microgrid was limited to the project team with two factor authentication upon log in.

An actual microgrid will operate in a very different environment that is harder to secure. $\mathrm{CN}$ nodes will be at the edge of the grid on utility poles or pad mounts that will be will need to be physically secured from intentional (e.g. vandalism) and unintentional access (e.g. pole damaged in traffic accident). Local area networking will likely be implemented over both hard-wired and wireless media. There will also be the requirement to add new components to and remove old components from the microgrid. Securing these "late joiners" will also require deployment of advanced cyber security concepts.

\subsection{Mapping to Legacy Protocols}

The implementations of the OpenFMB included configuring of adapters to legacy protocols such as DNP3 and Modbus. Configuration included mapping of the Global Data Space which is based on an information model to protocols that have no information model. The result is that the mappings are dependent on how the component vendor implemented the legacy protocols and could be different in each case (i.e. there are no standard register maps for these protocols).

This means that there will be a recurring cost to adding new types of components to OpenFMB implementations even though previously similar components have been added with the same protocol, but different scan maps. Greater adoption of OpenFMB in the marketplace may create incentives for vendors to provide native support in their components for the OpenFMB protocols (DDS, MQTT, and AMQP) and bring relief to these recurring costs.

\subsection{Form Factors}

Implementations of the OpenFMB must consider environmental factors when selecting components and form factors. This may be done by selecting equipment with the appropriate class of NEMA or IEEE ratings that match the environmental conditions required by the deployment. The forecasting work done at UTSA was deployed in Campbell Scientific Weatherproof Instrument Box that included a Hardkernel Odroid and a MyRio computer. The initial design used an enclosure created in-house that performed well in the lab test conditions, but encountered heat dissipation problems when employed in the field. This problem was remedied with a re-design of the $\mathrm{CN}$ that included an upgraded processor and the Campbell Scientific equipment housing.

\subsection{Microgrid as a Low Inertia Island}

In principle a microgrid may include different types of generation: Solar PV, Wind Power, Micro turbines, CHP, Diesels; different types of energy storage: BESS, Flywheels, and different types of loads: critical and shedable. During the design of the microgrid, attention must be paid to the selection of the 
types of resource and the associated variability of the generation and the variability of the load, and the capabilities of the energy storage device and the capabilities of their control systems.

The variability of the solar PV generation, wind generation and loads will lead to a requirement for fast acting "primary" reserve to keep the islanded microgrid operating within a narrow frequency band around $60 \mathrm{~Hz}$ to comply with requirements for quality of service and to prevent protective relaying devices from tripping inverters and other power system components off line. This primary reserve can be supplied by rotating inertia of loads, wind turbines, diesel generators, flywheels, and by smart inverter controllers as needed in the sub-second time frame.

In the testing at NREL ESIF, the simulated microgrid did not have an inertial component, unless the diesel generator was on line, as the inverters where not equipped with the advanced inertia mimicking controllers, and the wind power simulation was done with an electronic DC power source. With high variability of the simulated output from solar PV and wind power, the microgrid required the diesel generator to be on line and operating at mid-level power output to prevent tripping of the protective devices during periods of high variability.

As the project scope included testing of the concepts for interoperability, scheduling and dispatch but not the development and testing of new controllers for the sub second time frame, a workaround was found by configuring and loading the islanded microgrid with the diesel generator supplying inertial response. This experience reinforced the lesson that even though getting the data on variability of the output renewable resources presents a challenge, transient analysis studies over a horizon with varying renewable power inputs, are a required part of designing a microgrid that is expected to operate in islanded mode.

\subsection{Changes in Design and Operational Standards at Utilities}

The OpenFMB is intended, not to replace, but to co-exist with protective relaying schemes and to provide additional grid edge applications overlaid over existing relaying. However, there were noteworthy items that were observed during the project in the areas of requirements to make updates or changes in the design and operational standards at the utilities to which the microgrids are connected. Many existing utilities require microgrids to adopt protective relaying standards and practices that prevent the flow of energy from the microgrid back into the utility grid (no reverse power flow) under certain conditions, as described in IEEE Standard 1547 [1]. The project concept calls for power flows in both directions on the feeder as normal operating conditions. In these cases, protective relaying designs may be need to be changed to accommodate reverse power flow as a normal operating condition.

Similar concerns appear in the area of operational procedures and standards. The project concept allows for multiple microgrids with Distributed Energy Resources (DERs) connect to the grid, with power 
flowing in both directions. Operational procedures may need to be changed to accommodate these new flows. The project team at CPS Energy introduced several updates to their procedures for DMS dispatchers and linemen who monitor and manage the distribution grid to which the JBSA microgrid is connected as part of CPS Energy's change management procedures.

\subsection{Isolation Transformers for Inverters}

Another lesson learned by the project team concerns the requirement for external isolation transformers between some types of inverters and the utility grid. In the cases demonstrated in these projects, the transformers were from $3 \mathrm{XX}$ to 480 volts. These transformers are not usually in a utility's catalog of standard transformers and have to be special or custom ordered with long lead times. These lead times need to be known and built into the project schedule for implementing microgrids that need to be connect to the utility grid. 


\section{Summary and Conclusions}

In summary, the OMNETRIC Corp., Duke Energy, CPS Energy, and the University of Texas at San Antonio (UTSA) created a project team to execute the project NREL Integrate RCS-4-42326, Topic Area 3: Integrated Systems: "OpenFMB Reference Architecture Demonstration." The project included development and demonstration of concepts that will enable the electric utility grid to host larger penetrations of renewable resources. The project concept calls for the aggregation of renewable resources and loads into microgrids and the control of these microgrids with an implementation of the OpenFMB Reference Architecture. The project included validation at NREL and demonstration at two utility sites. The validation and all demonstrations included use of various aspects of the OpenFMB Architectural Framework, including the publish/subscribe pattern using the DDS and MQTT protocols and adapters to show interoperability between/among IED's that use diverse protocols.

New approaches to the forecasting of electric load demand and solar PV generation using ANN and SVM methodologies were demonstrated as part of the project. UTSA developed a low cost MET station that included weather instruments, a sky camera that was deployed on an IED at the edge of the grid in close proximity to the solar PV arrays. New forecasting algorithms based on neural networks, and the processing of images of the sky were tested and evaluated as part of this project.

The costs and the benefits of a microgrid were assessed using a variety of data inputs. Net present value and cost benefit ratios were computed and assessed. The conclusions of this study show the conditions under which a microgrid can be justified.

The project team validated these concepts at the NREL ESIF facility in Golden Colorado with the creation of a MW scale synthetic microgrid using the simulation capabilities of the lab.

The project also included utility field demonstrations at Duke Energy's Mt Holly facility in North Carolina. At this site the OpenFMB Architecture was used to integrate a distributed intelligence control system made up of coordinated optimal scheduling and dispatch at a slow control cycle, and solar smoothing with no reverse power flow on a fast control cycle.

At the CPS Energy site at the Joint Base San Antonio, the an implementation of the OpenFMB Architecture was demonstrated that integrated locally deployed forecasting functions with monitoring and control of a microgrid by a Siemens MGMS. 


\section{Table of Acronyms}

\begin{tabular}{|l|l|}
\hline & \\
\hline AMQP & Advanced Message Queuing Protocol \\
\hline ANN & Artificial Neural Networks \\
\hline ASE & \\
\hline ASOS & Automated Surface Observing System \\
\hline BESS & Battery Energy Storage System \\
\hline BMS & Battery Management System \\
\hline BTU & British Thermal Units \\
\hline CIM & Common Information Model \\
\hline CN & Communication Node \\
\hline COP & Current Operating Plan \\
\hline DDS & Data Distribution Services \\
\hline DHI & Diffuse Horizontal Irradiation \\
\hline DIP & Dual In-Line Package \\
\hline DNI & Direct Normal Irradiance \\
\hline DMS & Distribution Management System \\
\hline DSI & Direct Solar Irradiance \\
\hline EMM & Electricity Market Management System \\
\hline EMS & Energy Management System \\
\hline ESB & Enterprise Service Bus \\
\hline ESIF & Energy System Integration Facility \\
\hline FFNN & Feed Forward Neural Networks \\
\hline GDS & Global Data Space \\
\hline GHI & Global Horizontal Irradiance \\
\hline GLM & Generation and Load Management \\
\hline GOES & Geostationary operational environmental satellite \\
\hline GTB & Grid Tie Breaker \\
\hline HIS & Historical Information System \\
\hline HVAC & Heating Ventilation Air Conditioning \\
\hline IDL & Interface Description Language \\
\hline IEC & International Electrotechnical Commission \\
\hline IEEE & Institute of Electrical and Electronic Engineers \\
\hline IIC RA & Industrial Internet Consortium Reference Architecture \\
\hline loT & Internet of Things \\
\hline IP & Internet Protocol \\
\hline ISO & Independent System Operator \\
\hline & \\
\hline
\end{tabular}




\begin{tabular}{|l|l|}
\hline JBSA & Joint Base San Antonio \\
\hline kWh & Kilo Watt Hour \\
\hline LBNL & Lawrence Berkley National Laboratory \\
\hline MAC & Media access control \\
\hline MAPE & Mean Absolute Percent Error \\
\hline MDMS & Meter Data Management System \\
\hline MESA & Modular Energy Storage Architecture \\
\hline MET & Meteorological station \\
\hline MGMS & Microgrid Management System \\
\hline MIP & Mixed Integer Programming \\
\hline MPE & Mean Percent Error \\
\hline MQTT & Message Queuing Telemetry Transport \\
\hline NAESB & North American Standards Board \\
\hline NEMA & National Electrical Manufactures Association \\
\hline NetCDF & Network Common data form \\
\hline NDI & Non Direct Irradiance \\
\hline NMAE & Normalized Mean Absolute Error \\
\hline NREL & National Renewable Energy Laboratory \\
\hline NWP & Numerical Weather Prediction \\
\hline OMG & Object Management Group \\
\hline OpenFMB & Open Field Message Bus \\
\hline PAP & Priority Action Plan \\
\hline PCC & Point of Common Coupling \\
\hline PDCP & Packet Data Convergence Protocol \\
\hline PSIL & Power System Integration Laboratory \\
\hline PV & Photo Voltaic \\
\hline QoS & Quality of Service \\
\hline RBF & Radial Basis Function \\
\hline RDMS & Relational Database Management System \\
\hline RLC & Radio Link Control \\
\hline RMQ & Retail Market Quadrant \\
\hline RSF & Research Support Facility (buildings at NREL ESIF) \\
\hline RTO & Regional Transmission Operator \\
\hline RTU & Remote Terminal Unit \\
\hline SBC & Single Board Computer \\
\hline SCADA & Supervisory Control and Data Acquisition \\
\hline SGIP & Smart Grid Interoperability Panel \\
\hline SSE & Sum Squared Error \\
\hline STLF & Short Term Load Forecast \\
\hline
\end{tabular}




\begin{tabular}{|l|l|}
\hline STSPF & Short Term Solar Power Forecasting \\
\hline SVM & Support Vector Machine \\
\hline SVR & Support Vector Regression \\
\hline TSI & Total Sky Imager \\
\hline UML & Unified Modeling Language \\
\hline UDP & User Data Protocol \\
\hline UTSA & University of Texas San Antonio \\
\hline XSD & XML Schema Definition Language \\
\hline
\end{tabular}




\section{Appendices}

\subsection{Appendix A Use Case 1: Optimization of Microgrid}

\subsubsection{Optimization Use Case Background}

Two use cases were included in the project scope. The first use case deals with optimal scheduling and dispatch of a microgrid, both when the microgrid is connected to a larger utility grid, and when the microgrid is operated as an island. This use case was documented in the format of the IEC standard 62559-2, and delivered to the project as [1]. The following paragraphs summarize this use case.

\subsubsection{Optimization Use Case}

The optimization use case deals with normal daily operations of a microgrid: Day-Ahead Scheduling, and Intra-day Dispatching and Scheduling. In the Day-Ahead Scheduling, an initial set of schedules is set up for the next operating day. In the Intra-day Dispatching and Scheduling, these schedules are updated for the remainder of an operating day. In the Day-ahead scheduling, schedules are considered as an optimization variable. In the Intra-day Dispatching and Scheduling, flows to the external grid (aka interchange schedules) are considered to fixed constraints in the next $\mathrm{k}$ intervals $(1, \ldots, \mathrm{k})$, and optimization variables in the following $\mathrm{j}$ intervals $(\mathrm{k}+1, \ldots \mathrm{k}+\mathrm{j})$, with $\mathrm{k}$, and $\mathrm{j}$ as selectable parameters.

There are two parts to this use case: Day-Ahead and Intra-day.

\section{Day-Ahead Scheduling}

Several steps are followed:

1. Loads are forecasted for the day-ahead using load forecasting

2. Renewable power resource (solar, wind) schedules for the day-ahead are forecasted, using renewable power forecasts

3. MGMS optimizes the day-ahead plan and comes up with planned schedules for flows on the connection to the grid, and resource operating schedules for the day-ahead. The end user can select the objective function to be used during the optimization.

4. MGMS sends schedules to utility control center so that the utility can incorporate the microgrid operations in their own daily plan.

$\underline{\text { Intra-day Dispatching and Scheduling }}$ 
1. Loads are forecasted for the remainder of the day using load forecasting, with sub hour intervals

2. Renewable Power (solar, wind) schedules are forecasted for the remainder of the operating day, using renewable power forecasts

3. MGMS optimizes the remainder of the operating day and adjusts planned schedules for flows on the connection to the grid, and resource operating schedules for the remainder of the operating day. The end user can select the objective function to be used during the optimization

4. MGMS sends schedules to utility control center

\subsubsection{Testing of Use Case}

This use case was used as the basis for the workflows tested during the NREL validation and the Utility demonstrations. Not all aspects of the use case could be tested due to various limitation at the sites. For example the CPS Energy microgrid has only one controllable resource when it is operated as an island, which means that there are not enough control variables to conduct an optimization. Enough testing was done to allow an assessment to be made of the concepts under test. Further details were included in the respective test reports.

\subsection{Appendix B Economic Cost/Benefit}

\subsubsection{Background}

The costs and the benefits of establishing a microgrid made up of renewable resources and loads integrated with the OpenFMB were studied. Inputs to the studies were varied to represent different parameters seen in different regions of North America. Parameters that were varied included geographic (urban vs rural) and utility tariff (high, medium, low). The analysis also varied the configuration of the microgrid ranging from:

- No microgrid but feeder reinforcement

- Microgrid made up renewables and large BESS

- Microgrid made up renewable, moderately sized BESS, and diesel generator

While this study provides insight into the costs and benefit of a microgrid, it also provides additional insight into the relative costs of using a microgrid to supply highly available power to critical loads and allows comparison with a more traditional distribution engineering approach of supplying critical loads from multiple feeders. The study was published as a project document [2].

\subsubsection{Study Results}

The analysis took into account various cost and benefit streams to calculate the financial viability across three hypothetical scenarios including the Base Case, Alternative Case 1 and Alternative Case 2. All cases 
exemplify potential solutions to increasing end-user electricity delivery reliability. The Base Case incorporates all relative costs and benefits related to installation of supply from a second feeder to ensure high reliability. Alternative Case 1 incorporates relative costs and benefits in relation to a photovoltaic array plus battery storage microgrid enabling the end-user to island from the primary grid for upwards of $8 \mathrm{MWh}$ of backup service. Lastly, Alternative Case 2 depicts a hybrid approach that combines a photovoltaic array, battery storage for backup power for up to one hour, and a diesel reciprocating generator to satisfy further reliability needs. Each scenario was assessed relative to Rural versus Urban costs and also at varying levels of Avoided Cost of Capacity and Energy - $\$ 0.08 / \mathrm{kWh}, \$ 0.15 / \mathrm{kWh}$, and $\$ 0.20 / \mathrm{kWh}$. Open-source information and data gathered from a variety of industry sources was utilized to set relative cost levels and to estimate benefits. After each scenario was assessed and a Microsoft Excel financial model was built, the project's Net Present Value and Benefit-Cost Ratio were utilized to compare each project's viability to other scenarios.

Capital Expenditure across all scenarios is estimated to reach between $\$ 2.997$ million and \$10.151 million with Base Case, Rural Scenario as the most expensive scenario due to increased distance of feeder length and land acquisition costs. Alternative Case 1 and 2 take advantage of the beneficial Federal Investment Tax Credit and Federal Bonus depreciation due to the photovoltaic arrays and battery storage which helps to drive down overall up-front cost. Alternative Case 1 Capital Expenditures reach upwards of \$7.326 million and Alternative Case 2 Capital Expenditures reach upwards of $\$ 3.560$ million. Total estimated project operating revenue from Grid Benefits range from $\$ 10.492$ thousand to $\$ 12.852$ million across all cases. The Base Case shows the least revenue creation at orders of magnitude less than Alternative Case 1 and 2. Alternative Case 1 benefits reach upwards of 12.852 million, whereas Alternative Case 2 reaches total project revenues of up to $\$ 8.383$ million. Except for years that photovoltaic inverters and battery storage need replacement, Alternative Case 1 and 2 do create positive revenue, with Alternative Case 1 reaching a high of $\$ 913,819$ in year twenty-five.

Alternative Case 2 shows the highest project Net Present Value reaching upwards of $\$ 1.862$ million. All Alternative 1 and 2 scenarios with Avoided Costs of Energy and Capacity over and including \$0.15/kWh reach a positive Net Present Value. No Base Case scenario reaches a positive Net Present Value. Please refer to Table 2 below for scenarios with the highest Net Present Value.

\begin{tabular}{cc}
\hline \multicolumn{1}{c}{ Scenario } & Net Present Vale \\
\hline Alternative 2 , Urban $-\$ 0.20 / \mathrm{kWh}$ & $\$ 1,862,779.92$ \\
\hline Alternative 2 , Rural $-\$ 0.20 / \mathrm{kWh}$ & $\$ 1,837,244.42$ \\
Alternative 1 , Urban $-\$ 0.20 / \mathrm{kWh}$ & $\$ 1,224,034.53$ \\
Alternative 1 , Rural $-\$ 0.20 / \mathrm{kWh}$ & $\$ 1,190,568.09$ \\
\hline Alternative 2, Urban $-\$ 0.15 / \mathrm{kWh}$ & $\$ 842,708.22$ \\
\hline
\end{tabular}


The Benefit-Cost Ratio indicates resources are not well invested in the Base Case as compared to alternative cases. Alternative Case 2 Benefit-Cost ratios tend to outperform Alternative Case 1 by upwards of twenty percent. Alternative Case 2 shows the highest ratio of 1.77 across all scenarios when Avoided Cost of Energy and Capacity are $\$ 0.20$ per kWh and costs are within an urban setting. Additionally, Alternative Case 2 allocates up to forty-eight percent less capital to achieve these ratios. The Base Case reaches Benefit-Cost Ratio levels at orders of magnitude smaller than alternative cases, reaching its highest ratio of 0.008 . Refer to Table 3 for the five scenarios with the highest Benefit-Cost Ratios.

\begin{tabular}{cc}
\multicolumn{1}{c}{ Scenario } & Benefit-Cost Ratio \\
\hline Alternative 2 , Urban $-\$ 0.20 / \mathrm{kWh}$ & 1.77 \\
Alternative 2 , Rural $-\$ 0.20 / \mathrm{kWh}$ & 1.75 \\
Alternative 2 , Urban $-\$ 0.15 / \mathrm{kWh}$ & 1.45 \\
Alternative 2 , Rural $-\$ 0.15 / \mathrm{kWh}$ & 1.44 \\
Alternative 1, Urban $-\$ 0.20 / \mathrm{kWh}$ & 1.42 \\
\hline
\end{tabular}

Table 14: Scenarios with Highest Cost Benefit Ratios

Social Benefits including the Reliability Value to End-User, Social Cost of Carbon, and various avoided emissions due to photovoltaic energy production were calculated. The Reliability Value to End-User reached a Net Present Value of $\$ 9,140,808.49$ over the lifetime of the project for all cases and scenarios. Additionally, it is estimated Scenario 1 and 2 create significant environmental benefits due to clean energy production across all scenarios - Scenario 1 slightly out performs Scenario 2 regarding avoided emissions due larger PV-charged battery storage capacity and no diesel backup generation. Refer to Table 4 for the calculated social benefits across all scenarios. Social Benefits including Reliability Value to End-User and Avoided Social Cost of Carbon were not included in cash flow modeling and do not impact project Net Present Value or Benefit-Cost Ratio results.

\begin{tabular}{|c|c|c|c|c|c|}
\hline Alternative & $\begin{array}{c}\text { Reliability Value to } \\
\text { End-User }\end{array}$ & $\begin{array}{l}\text { Avoided } \\
\text { Sulfur Dioxide } \\
\text { (Metric Tons) }\end{array}$ & $\begin{array}{c}\text { Avoided Nitrogen } \\
\text { Oxide } \\
\text { (Metric Tons) }\end{array}$ & $\begin{array}{c}\text { Avoided Carbon } \\
\text { Dioxide } \\
\text { (Metric Tons) }\end{array}$ & $\begin{array}{c}\text { Avoided Social } \\
\text { Cost of Carbon } \\
\text { (Net Present } \\
\text { Value) }\end{array}$ \\
\hline $\begin{array}{c}\text { Base Case - All } \\
\text { Scenarios }\end{array}$ & $\$ 9,140,808.49$ & $x$ & $x$ & $x$ & $x$ \\
\hline
\end{tabular}




\begin{tabular}{|c|c|c|c|c|c|}
\hline $\begin{array}{c}\text { Alternative } 1 \text { - All } \\
\text { Scenarios }\end{array}$ & $\$ 9,140,808.49$ & 31.65 & 19.78 & $19,677.33$ & $\$ 172,982.43$ \\
\hline $\begin{array}{c}\text { Alternative } 2 \text { - All } \\
\text { Scenarios }\end{array}$ & $\$ 9,140,808.49$ & 31.55 & 19.71 & $19,614.75$ & $\$ 172,434.83$ \\
\hline
\end{tabular}

Table 15: Social Benefits for All Cases and Scenarios over Analysis Time Horizon of 25 years

\subsection{Appendix C Press Releases}

NREL ESIF and Duke Testing

CPS Energy Testing 


\section{References}

\section{Section 1 References}

None

\section{Section 2 References}

None

\section{Section 3 References}

None

\section{Section 4 References}

Section 4 References

[1] Distributed Intelligence Platform Reference Architecture Vol 1: Vision and Overview

[2] SGIP resource link

[3] NAESB RMQ.26 OpenFMB Model Business Practice

[4] Industrial Internet Consortium: Industrial Internet Reference Architecture

[5] J. Day, H. Zimmermann, “The OSI Reference Model” The Proceedings of the IEEE Vol 71, No 12, December 1983

[6]IEC 61970-301

[7] IEC 61968-13

[8] IEC 62325-301

[9] SGIP webinar on OpenFMB

[10] IEC 62599-2 Use Case methodology Part 2: Definition of templates for use cases, actor list and requirements list

[11] SGIP repository (tested)

[12] Cimtool

[13] SGIP Annual Meeting, New Orleans 2015, link to site [14]Distributech 2016 Orlando, Florida 
[15] OMNETRIC Costs and Benefits of Implementing a Microgrid

[16] NERC CIP version 5

[17] SWTI security document

[18] SP7 MGMS BSS Cyber Security, Annex X

\section{Section 5 References}

[1]Abushakra B. An inverse model to predict and evaluate the energy performance of large commercial and institutional buildings. In: Building simulation vol. 3; 1997. p. 403-10.

[2].- Kissock K, Haberl JS, Claridge DE. Development of a toolkit for calculating linear, change point linear and multiple-linear inverse building energy analysis models. Final Report for ASHRAE Research Project, No. 1050-RP. Energy Systems Laboratory; 2002. Report No. ESLTR-02-11-02.

[3].- Braun JE, Chaturvedi N. An inverse gray-box model for transient building load prediction. HVAC\&R Res 2002;8(1):73-99.

[4[.- Dong B, Cao C, Lee SE. Applying support vector machines to predict building energy consumption in tropical region. Energy Build 2005;37(5):545-53.

[5].- Karatasou S, Santamouris M, Geros V. Modeling and predicting building's energy use with artificial neural networks: methods and results. Energy Build 2006;38(8):949-58.

[6].- Mui KW, Wong LT, Law LY. An energy benchmarking model for ventilation systems of airconditioned offices in subtropical climates. Appl Energy 2007;84(1):89-98.

[7].- Heo Y, Zavala VM. Gaussian process modeling for measurement and verification of building energy savings. Energy Build 2012;53:7-18.

[8].- Granderson J, Price P. Evaluation of the predictive accuracy of five whole building baseline models. Berkeley, CA: Lawrence Berkeley National Laboratory; 2012. Tech. Rep. LBNL-5886E, August 2012.

[9]. -Yoshida H, Kumar S, Morita Y. Online fault detection and diagnosis in VAV air handling unit by RARX modeling. Energy Build 2001;33(4):391-401.

[10].- Lee KH, Braun JE. Development and application of an inverse building model for demand response in small commercial buildings. Proc SimBuild 2004: 1-12. 
[11].- Li P, O'Neill ZD, Braun JE. Development of control-oriented models for model predictive control in buildings. Ashrae Trans 2013;119(2).

[12].- Claridge DE. Using simulation models for building commissioning. In: International conference on enhanced building operation, energy systems laboratory, Texas A\&M University; 2004, October.

[13].- Thomas Ng S, Skitmore M, Wong KF. Using genetic algorithms and linear regression analysis for private housing demand forecast. Build Environ 2008;43(6):1171-84.

[14].- Kwok SS, Yuen RK, Lee EW. An intelligent approach to assessing the effect of building occupancy on building cooling load prediction. Build Environ 2011;46(8):1681-90.

[15].- Kissock JK. Modeling commercial building energy use with artificial neural networks. In: Intersociety energy conversion engineering conference vol. 3; 1994. p. 1290e5.

[16].- Krarti M, Kreider JF, Cohen D, Curtiss P. Estimation of energy savings for building retrofits using neural networks. J Sol Energy Eng 1998;120 (3):

[17].- Dhar A, Reddy TA, Claridge DE. Generalization of the fourier series approach to model hourly energy use in commercial buildings. J Sol Energy Eng 1999;121(1):54-62.

[18].- Solomon DM, Winter RL, Boulanger AG, Anderson RN, Wu LL. Forecasting energy demand in large commercial buildings using support vector machine regression. Department of Computer Science, Columbia University; 2011. Tech. Rep. CUCS-040-11, September 2011.

[19].- Zhang Y, O'Neil Z, Wagner T, Augenbroe G. An inverse model with uncertainty quantification to estimate the energy performance of an office building. In: Proceedings of 13th international building performance simulation association conference; 2013.

[20].- Srivastav A, Tewari A, Dong B. Baseline building energy modeling and localized uncertainty quantification using Gaussian mixture models. Energy Build 2013;65:438-47.

[21].- Diange M, David M, Lauret P, Boland J, Schmutz N. Review of solar irradiance forecasting methods and a proposition for small-scale insular grids. Renewable and Sustainable Energy Reviews 27 , 65-76 (2013).

[22].- Bacher P, Madsen H, Nielsen HA, Online short-term solar power forecasting. Solar Energy 83, 1772-1783 (2009).

[23].- Mathiesen P, Kleissl J. Evaluation of numerical weather prediction for intra-day solar forecasting in the continental United States. Solar Energy 85, 967-977 (2011).

[24].- Huang J, Korolkiewicz M, Agrawal M, Boland J. Forecasting solar radiation on an hourly time scale using a Coupled AutoRegressive and Dynamical System (CARDS) model. Solar Energy 87, 136149 (2013). 
[25].- Chow CW, Urquhart B, Lave M, Dominguez A, Kleissl J, Shields J, Washom B. Intro-hour forecasting with a total sky imager at the UC San Diego solar energy testbed. Solar Energy 85, 2881-2893 (2011).

[26].- Chia-Lin F, Cheng HY. Prediction of solar irradiance with all-sky image features via regression. Solar Energy 97, 537-550 (2013).

[27].- Quesada-Ruiz S, Chu Y, Tovar-Pescador J, Pedro HTC, Coimbra CFM. Cloud-tracking methodology for intra-hour DNI forecasting. Solar Energy 102, 267-275 (2014).

J. Nummikoski, Y. S. Manjili, R. Vega, and H. Krishnaswami, “ADAPTIVE RULE GENERATION FOR SOLAR FORECASTING: INTERFACING WITH A KNOWLEDGE-BASE LIBRARY”, Proc. to IEEE 39th Photovoltaic Specialists Conference (PVSC), 2013 Tampa, FL, USA.

B. Dong, Z. Li, S.M.M. Rahman, R. Vega. A hybrid model approach for forecasting future residential electricity consumption Energy and Buildings 117, 341-351 (2016).

Z. Li, S.M.M. Rahman, R. Vega, B. Dong. A hierarchical approach using machine learning methods in solar photovoltaic energy production forecasting. Energies 9, 55 (2016).

M. Cervantes, H. Krishnaswami, W. Richardson, R. Vega. Utilization of Low Cost, Sky-Imaging Technology for Irradiance Forecasting of Distributed Solar Generation. 2016 IEEE Green Technologies Conference (GreenTech). Pages 142 - 146. Kansas City, MO. 6-8 April 2016.

\section{Section 6 References}

[1] Spectrum Power ${ }^{\mathrm{TM}}$ MGMS v1.30 Application Sheet

\section{Section 7 References}

[1] OMNETRIC Architecture Description MGMS-Open Field Message Bus

[2] OMNETRIC, Detailed Design NREL Information Exchange Modeling

[3] Siemens MGMS Data Collection Guide

[4] OMNETRIC Frame Test Specification NREL Integrate Program

[5] OMNETRIC Test Book NREL MGMS

[6] OMNETRIC Test Book Forecasting

[7] OMNETRIC Test Results 
[8] Real Time Innovations DDS Implementation

[9] Apache Foundation MQTT

\section{Section 8 References}

[1] OMNETRIC Design Document; Mt Holly Microgrid

[2] OMNETRIC Design Document: Demonstration of Distributed Control at Mt Holly

[3] OMNETRIC Test Book Duke MGMS Workflows

[4] OMNETRIC Test Results Duke MGMW Workflows

[5] www.wunderground.com

\section{Section 9 References}

[1] OMNETRIC Design Document: MGMS at Joint Base San Antonio

[2] OMNETRIC Test Book: CPS MGMS

[3] OMNETRIC Test Results

[4] Real Time Innovations DDS

\section{Section 10 References}

[1] Industrial Internet Reference Architecture

[2] IEC 61970

[3] IEC 61968

[4] IEC 62325

[5] IEC 61850

[6] Common Criteria for Information Technology Security Evaluation (ISO/IEC 15408)

[7] Federal Information Processing Standard Publication, NIST Publication 140-2

[8] NAESB RMQ.26 OpenFMB 


\section{Section 11 References}

None

\section{Section 12 Reference}

None

Section 13 References

None 